Stoll, Bettina [Hrsg.]; Herrmann, Heike [Hrsg.]

\title{
Corporate Social Responsibility - Impulse aus der und für die Profit- und Sozialwirtschaft
}

Opladen; Berlin; Toronto : Verlag Barbara Budrich 2020, 139 S. - (Gesellschaft und Nachhaltigkeit; 7)

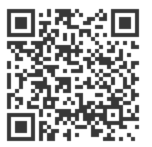

Quellenangabe/ Reference:

Stoll, Bettina [Hrsg.]; Herrmann, Heike [Hrsg.]: Corporate Social Responsibility - Impulse aus der und für die Profit- und Sozialwirtschaft. Opladen; Berlin; Toronto : Verlag Barbara Budrich 2020, $139 \mathrm{~S}$.

(Gesellschaft und Nachhaltigkeit; 7) - URN: urn:nbn:de:0111-pedocs-207608 - DOI: 10.25656/01:20760

https://nbn-resolving.org/urn:nbn:de:0111-pedocs-207608

https://doi.org/10.25656/01:20760

in Kooperation mit / in cooperation with:

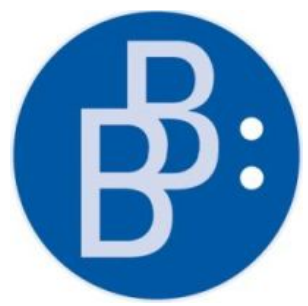

https://www.budrich.de

\section{Nutzungsbedingungen}

Dieses Dokument steht unter folgender Creative Commons-Lizenz: http://creativecommons.org/licenses/by/4.0/deed.de - Sie dürfen das Werk bzw. den Inhalt vervielfältigen, verbreiten und öffentlich zugänglich machen sowie Abwandlungen und Bearbeitungen des Werkes bzw. Inhaltes anfertigen, solange Sie den Namen des Autors/Rechteinhabers in der von inm festgelegten Weise nennen.

Mit der Verwendung dieses Dokuments erkennen Sie die Nutzungsbedingungen an.

\section{Terms of use}

This document is published under following Creative Commons-License: $\mathrm{http}: / /$ creativecommons.org/licenses/by/4.0/deed.en - You may copy, distribute and render this document accessible, make adaptations of this work or its contents accessible to the public as long as you attribute the work in the

By using this particular document, you accept the above-stated conditions of use.

\section{(c) (†)}

\section{Kontakt / Contact:}

\section{peDOCs}

DIPF | Leibniz-Institut für Bildungsforschung und Bildungsinformation

Informationszentrum (IZ) Bildung

E-Mail: pedocs@dipf.de

Internet: www.pedocs.de

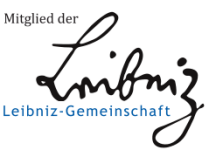


Bettina Stoll I Heike Herrmann (Hrsg.)

\title{
Corporate Social Responsibility - Impulse aus der und für die Profit- und Sozialwirtschaft
}

\author{
Verantwortung \\ und Nachhaltigkeit
}


Corporate Social Responsibility

- Impulse aus der und für die

Profit- und Sozialwirtschaft 


\section{Buchreihe}

Gesellschaft und Nachhaltigkeit

herausgegeben von

Prof. Dr. Monika Alisch

Prof. Dr. Stefanie Deinert

Prof. Dr. Martina Ritter

Prof. Dr. Bettina Stoll

Band 7 
Bettina Stoll

Heike Herrmann (Hrsg.)

\section{Corporate Social Responsibility \\ - Impulse aus der und für \\ die Profit- und Sozialwirtschaft \\ Verantwortung und Nachhaltigkeit}

Verlag Barbara Budrich

Opladen • Berlin • Toronto 2020 
Bibliografische Information der Deutschen Nationalbibliothek

Die Deutsche Nationalbibliothek verzeichnet diese Publikation in der Deutschen

Nationalbibliografie; detaillierte bibliografische Daten sind im Internet über

https://portal.dnb.de abrufbar.

(C) 2020 Dieses Werk ist bei der Verlag Barbara Budrich GmbH erschienen und steht unter der Creative Commons Lizenz Attribution 4.0 International

(CC BY 4.0): https://creativecommons.org/licenses/by/4.0/

Diese Lizenz erlaubt die Verbreitung, Speicherung, Vervielfältigung und Bearbeitung unter Angabe der UrheberInnen, Rechte, Änderungen und verwendeten Lizenz. www.budrich.de

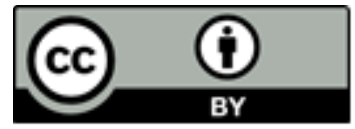

Dieses Buch steht im Open-Access-Bereich der Verlagsseite zum kostenlosen Download bereit (https://doi.org/10.3224/84742400).

Eine kostenpflichtige Druckversion kann über den Verlag bezogen werden. Die Seitenzahlen in der Druck- und Onlineversion sind identisch.

$$
\begin{array}{ll}
\text { ISBN } & 978-3-8474-2400-0 \text { (Paperback) } \\
\text { eISBN } & 978-3-8474-1584-8 \text { (PDF) } \\
\text { DOI } & 10.3224 / 84742400
\end{array}
$$

Umschlaggestaltung: Bettina Lehfeldt, Kleinmachnow - www.lehfeldtgraphic.de Titelbildnachweis: Foto: Philipp Lehfeldt

Typografisches Lektorat: Anja Borkam, Jena - kontakt@lektorat-borkam.de Druck: Books on Demand GmbH, Norderstedt

Printed in Europe 


\section{Vorwort der Reihenherausgeberinnen}

In der Diskussion um Nachhaltigkeit hat sich längst eine Sichtweise durchgesetzt, welche die drei Dimensionen von Nachhaltigkeit - sozial, ökologisch und ökonomisch - als unteilbar miteinander verbunden erkannt hat und stärker die Wechselwirkungen und Zielkonflikte in den Blick nimmt.

Die angestrebte Gleichzeitigkeit und Gleichwertigkeit und das Ausbalancieren der drei Nachhaltigkeitsdimensionen ist die große gesellschaftliche Herausforderung und kann in einer hoch differenzierten Gesellschaft nur dann gelingen, wenn die Vielfalt der Perspektiven ausreichend zum Tragen kommt und entsprechende Institutionen kooperativ und kommunikativ ihre Ressourcen auf diesen Anspruch ausrichten.

Als Bildungseinrichtung sieht sich die Hochschule für angewandte Wissenschaften in Fulda gefordert, diese Herausforderung anzunehmen und in der Forschung nicht nur interdisziplinär, sondern transdisziplinär aufzugreifen. Dazu hat die Hochschule im Jahr 2011 das wissenschaftliche Zentrum „Gesellschaft und Nachhaltigkeit - Centre of Research for Society and Sustainability (CeSSt) gegründet, in dem sich über fünfzig Wissenschaftler*innen aus verschiedensten Fachgebieten für einen wissenschaftlichen Austausch und die Entwicklung entsprechender Forschungsvorhaben zusammengetan haben.

Die Schriftenreihe „Gesellschaft und Nachhaltigkeit“ dokumentiert die Arbeit des Zentrums und sensibilisiert für eine sozialverträgliche Entwicklung in unterschiedlichen gesellschaftlichen Handlungsfeldern. Gefragt wird, ,was' zu tun ist, aber auch, wie ' gesellschaftliche Verantwortung umgesetzt werden kann.

Die Beiträge der seit dem Jahr 2012 erscheinenden Schriftenreihe befassen sich nicht nur mit Fragen der Befriedigung von Grundbedürfnissen zur Reduzierung von (globaler) Armut, sondern auch mit denen der Entwicklung von Humankapital beispielsweise durch Bildung oder Gesundheitsversorgung und adressieren neben diesen auf materielle und immaterielle Ressourcen gerichteten Herausforderungen auch die Gender- und Generationengerechtigkeit, sozialräumliche Disparitäten und die Operationalisierung von Menschenrechten durch Prozesse von Teilhabe.

Fulda, Januar 2020

Monika Alisch, Stefanie Deinert, Martina Ritter, Bettina Stoll 



\section{Inhaltsverzeichnis}

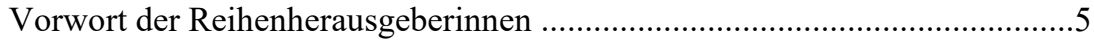

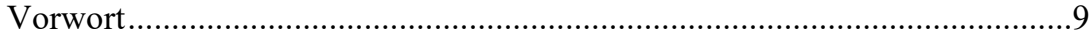

Bettina Stoll und Heike Herrmann

Was Corporate Social Responsibility ist...

hängt von ihrer Rahmung ab

Heike Herrmann

Gesellschaftlichen Herausforderungen mit Sozialen Kooperationen

begegnen

Karl-Hans Kern

Gemeinsam mehr bewegen - Erfolgsfaktoren für Kooperationen

zwischen sozialen Organisationen und Wirtschaftsunternehmen in Zeiten

von CSR

Bettina Stoll

Ansätze zur Verankerung von CSR in profit- und sozialwirtschaftlichen Unternehmen

\section{Christoph Schleer}

Corporate Social Responsibility als Instrument der Mitarbeitergewinnung:

Gründe für die gestiegene Relevanz von CSR und zielgruppenspezifische

CSR-Kommunikation mit den Sinus-Milieus ${ }^{\circledR}$ 
Maria Riegler und Markus Scholz

Entstehungsfaktoren von Collective-Action-Initiativen zur Lösung sozialer und ökologischer Probleme.

Thomas Rusche

Methodische Schärfung der CSR-Diskussion .............................................105

Roundtable am 29. Mai 2018

Chancen und Herausforderungen von CSR in der Sozialwirtschaft und Sozialen Organisationen

Angaben zu den Autorinnen und Autoren 


\section{Vorwort}

Corporate Social Responsibility als soziale bzw. gesellschaftliche Verantwortung von Unternehmen (synonym: ,unternehmerische Nachhaltigkeit") ist ein in diverser Hinsicht extrem facettenreich verwendeter Begriff. Es besteht inzwischen ein breites Interesse unterschiedlicher Wissenschaften, etwa der Wirtschafts- und Umweltwissenschaften, ebenso wie der Gesellschafts- bzw. Sozialwissenschaften (z.B. Politikwissenschaft, Soziologie) bezüglich CSRbezogener Fragestellungen und ihrer Klärung. Der vorliegende Band nimmt diese Perspektivenvielfalt auf und bewegt sich mit seinen Beiträgen maßgeblich in und verbindend zwischen den Wirtschafts- und Sozialwissenschaften. Dabei profitiert der Band von den verschiedenen und gleichzeitig verbindenden Kontexten und Sichtweisen der Autor*innen. Anstoß zum Band war die Realisierung eines einjährigen Forschungsprojektes der beiden Herausgeberinnen im Rahmen des Hessischen Programmes (Ministerium für Wissenschaft und Kunst) „Forschung für die Praxis“ unter dem Titel „CSR bzw. Corporate Citizenship - Potenziale zur Stärkung der (sozialen) Teilhabe von Menschen (und Gruppen)“. Dem Forschungsprojekt folgte u.a. eine Tagung, welche CSR einerseits durch die Verbindung von Wirtschaft und Sozialem durch „Soziale Kooperationen“ (Stichwort: Corporate Citizenship) in den Fokus nahm, aber darüber hinaus CSR als Leitkonzept/-idee sowohl in der Profitwirtschaft als auch der Sozialwirtschaft diskutierte. Wir freuen uns, dass die Tagungsreferent*innen mit Ihren Beiträgen in diesem Band gemeinsam mit uns die hieraus entstandenen „Impulse aus der und für die Profit- und Sozialwirtschaft“ in Sachen CSR zur Diskussion stellen.

Fulda, Januar 2020

Bettina Stoll und Heike Herrmann 



\title{
Was Corporate Social Responsibility ist... hängt von ihrer Rahmung ab
}

\author{
Bettina Stoll und Heike Herrmann
}

Im Grünbuch der Europäischen Union aus dem Jahr 2001 wird CSR als ein Konzept bezeichnet, ,das den Unternehmen als Grundlage dient, auf freiwilliger Basis soziale Belange und Umweltbelange in ihre Unternehmenstätigkeit und in die Wechselbeziehungen mit den Stakeholdern zu integrieren" (Europäische Kommission 2001: 7). Zehn Jahre später bezog sich die EU-Kommission explizit auf ,die Verantwortung von Unternehmen für ihre Auswirkungen auf die Gesellschaft" (Europäische Kommission 2011: 7) und wurde gleichzeitig verbindlicher, indem sie rechtliche Maßnahmen ankündigte. Mit der sogenannten CSR-Richtlinie wurden wenige Jahre später Unternehmen dazu verpflichtet, über die sozialen und ökologischen Folgen ihres Tuns zu informieren (Richtlinie 2014/95/EU; über das CSR-Richtlinie-Umsetzungsgesetz in deutsches Recht überführt; vgl. BMJV 2017). Es wird das Ziel formuliert, unternehmerisches Handeln ,umweltverträglich, ethisch und sozial verantwortlich und zugleich ökonomisch erfolgreich - kurz nachhaltig“ zu gestalten (vgl. Kropp 2019: 38; vgl. Lexikon der Nachhaltigkeit 2016). Dies erklärt einen wesentlichen Teil des Titels dieses Bandes.

Was genau und differenziert unter dem Begriff und „Konzept“ Corporate Social Responsibility zu thematisieren und diskutieren ist, hängt stark davon $\mathrm{ab}$, in welchem Kontext - welcher Rahmung - diese behandelt wird. Eine erste relevante Rahmung ergibt sich z.B. aus der Frage, ob der Fokus der Verantwortung primär auf die Wirkung des Unternehmens nach außen, auf die Gesellschaft, oder primär auf dessen Wirkung auf die interne Wertschöpfungskette und die internen Stakeholder des Unternehmens gelegt wird. Unter Bezug auf die eher nach außen, auf das gesellschaftliche Umfeld, gerichtete Dimension des Begriffs CSR ${ }^{1}$ ist festzustellen: Während Unternehmen der Profitwirtschaft unter dem Stichwort CSR als Corporate Citizen Probleme ihres gesellschaftlichen Umfeldes angehen und darum bemüht sind, neben dem Streben nach Gewinn auch ökologische und soziale Belange zu berücksichtigen, sind

1 Zur Unterscheidung einer internen und nach außen gerichteten Dimension von CSR vgl. Riess 2010: 589. 
Unternehmen der Sozialwirtschaft scheinbar per se „sozial“ und gemeinnützig unterwegs, gilt es hier die gesellschaftsrelevante ökonomische und die ökologische Dimension im unternehmerischen Handeln stärker zu berücksichtigen. In Bezug auf beide Unternehmensformen (Profit- wie Sozialwirtschaft) sind diesbezüglich jeweils stattfindende Verkürzungen des Begriffs festzustellen. Zusätzlich zu dieser bestehenden Verkürzung in der Diskussion um CSR lässt sich eine weitere in der sozialwirtschaftlichen Praxis verorten. Hier wird CSR häufig vor allem im Zusammenhang mit Sozialen Kooperationen/Partnerschaften mit der Profitwirtschaft diskutiert.

Auch in der zweiten innerbetrieblichen Dimension des Begriffs CSR (sie bezieht sich z. B. auf den Umgang mit Mitarbeiterinnen und Mitarbeitern, die Produktion von Emissionen im Wertschöpfungsprozesses oder das Lieferketten- bzw. Dienstleistungsmanagement mit den daraus resultierenden internen wie externen Auswirkungen auf Menschen und Umwelt) wird den Unternehmen der Sozialwirtschaft häufig ein soziales und Gemeinwohl orientiertes Handeln unterstellt, werden innerbetriebliche Strukturen und Prozesse selten unter dem Begriff CSR diskutiert. Auch hierbei handelt es sich jedoch um eine Verkürzung, sind innerbetriebliche Prozesse und Strukturen sowohl in der Profit- wie in der Sozialwirtschaft unter dem Gesichtspunkt der CSR zu diskutieren.

Davon ausgehend, dass gesellschaftliche Verantwortung sowohl Profitwirtschaft als auch Sozialwirtschaft in allen drei Dimensionen der Nachhaltigkeit (ökonomisch, ökologisch und sozial) betrifft, macht es keinen Sinn, in einer eindimensionalen oder nur eine Perspektive (Profit- oder Sozialwirtschaft) berücksichtigen Position zu verbleiben. Vielmehr geht es genau darum, die Dimensionen und Perspektiven in ihrem Wirkungsgefüge zu diskutieren, $d$. h. mehrperspektivisch und mehrdimensional zu denken. In diesem Sinne sind die Beiträge des vorliegenden Bandes mit Impulsen aus der und für die Profit- und Sozialwirtschaft ein erster Schritt, um die unterschiedlichen Dimensionen und Perspektiven zusammen zu bringen.

Mit den unterschiedlichen Wirtschaftskontexten (Profit-/Sozialwirtschaft) ist gleichzeitig eine weitere wichtige Rahmung angesprochen, welche CSR in einem jeweils spezifischen „Licht erscheinen“ lässt/erscheinen lassen kann. Weitere relevante Rahmungen werden mit den folgenden Abschnitten aufgegriffen, so etwa vertiefend der Aspekt der „Nachhaltigkeit als Kontext/Rahmung von CSR“, „CSR als letztliches Ergebnis von Aushandlungsprozessen und Kooperation“, „CSR im Rahmen von Unternehmen unterschiedlicher Größe“ oder „CSR verstanden als „Treiber“ von Entwicklungsprozessen in Organisationen". 


\section{CSR - Eine Frage der Profit- UND der Sozialwirtschaft?}

Die gesellschaftliche Verantwortung von Unternehmen bzw. unternehmerische Nachhaltigkeit (Corporate Social Responsibility) wird in Theorie und Praxis bisher hauptsächlich sichtbar im Kontext profitorientierter Unternehmen diskutiert. Der „Reflex“ Organisationen bzw. Unternehmen der Sozialwirtschaft (intern oder extern veranlasst) normativ, strategisch und operativ auf den Verantwortungs- bzw. Nachhaltigkeitsprüfstand zu stellen ist bisher weit weniger ausgeprägt als in und gegenüber klassischen Wirtschaftsunternehmen. Suboptimale gesellschaftliche Auswirkungen bzw. Effekte der profitwirtschaftlichen Unternehmen sind öffentlich sicht- und greifbarer und haben weltbzw. europaweit bereits zu einer langjährigen Diskussion von CSR geführt.

Eine der zentralen Grundannahmen im Kontext der bis dato geführten „CSR-Debatte“ dürfte dabei sein, dass die Orientierung am Profit als Sinn und Zweck der Unternehmen dazu führt, dass soziale und ökologische Aspekte des Unternehmens dem Streben nach Gewinnmaximierung zum Opfer fallen (s. auch Rusche in diesem Band), gesellschaftlich verantwortliches bzw. nachhaltiges Handeln im und außerhalb des Unternehmens damit nur bedingt möglich ist. Kann daraus im Umkehrschluss davon ausgegangen werden, dass sozialwirtschaftliche Unternehmen, ausgerichtet an sozialen Zwecken/am Gemeinwohl, per se nachhaltig aufgestellt sind, sich in einer sozial-ökonomisch-ökologischen Balance befinden? Ohne dass „die Sozialwirtschaft“ bis dahin und diesbezüglich systematisch beforscht wurde, lässt sich - auch auf Basis deren eigener Einschätzung (vgl. Hofmacher 2017: 36, Eichmann 2016: 30) - behaupten: Nein. Aktuell als Handlungsfelder von CSR eingestufte, ausgehandelte Bereiche (z.B. Gesundheit und Sicherheit am Arbeitsplatz; nachhaltige Nutzung von Ressourcen; verantwortliche Gestaltung der Beschäftigung und Beschäftigungsverhältnisse) und deren Betrachtung im sozialwirtschaftlichen unternehmerischen Kontext zeigen, dass auch diese Unternehmen in CSR-Bereichen und in deren Zusammenhang nicht optimal aufgestellt sind. Dass ein Unternehmen durch einen sozialen, gemeinnützigen Zweck in seiner Existenz berechtigt ist, führt im Resultat nicht dazu, dass es sich umfassend in einer optimalen ökologisch - ökonomisch - sozialen Balance befindet. Dass aber per se CSR bzw. unternehmerische Nachhaltigkeit auch in den sozialwirtschaftlichen Unternehmen von Bedeutung ist bzw. Leitziel sein sollte und in bestimmten Kontexten ein generelles Bewusstsein dafür vorhanden ist, wird u.a. deutlich beim Blick auf Managementsysteme bzw. Initiativen, die auf gesellschaftlich verantwortliches Handeln gerichtet sind. (International) entwickelte und diskutierte Systeme und Initiativen, die auf CSR zielen, wie die Global Reporting Initiative, EMAS PLUS, ISO 26.000 (zu EMAS PLUS und ISO 26000: s. auch Stoll in diesem Band) beziehen sich explizit auch auf Nicht- 
regierungs- bzw. Not-for-Profit-Organisationen. Allerdings ist davon auszugehen, dass sich die Frage nach der Balance und Ausgestaltung der Dimensionen und Handlungsfelder auf Basis eines jeweils unterschiedlichen Ausgangsniveaus/-punkte(s) vollzieht. Der „Auftrag“ und Herausforderung bleibt bzw. ist jedoch für Profit- und Sozialwirtschaft bzw. ihre Unternehmen und Organisationen ,gleich“: Die Orientierung am „Leitbild“ CSR bzw. Nachhaltigkeit. Daher werden mit diesem Band auch explizit Impulse für die CSR in sozialwirtschaftlichen Organisationen gesetzt.

\section{CSR - eine Frage der Nachhaltigkeit}

Die EU-Kommission bzw. die öffentliche Hand setzte über die gesetzliche Aufforderung zur Berichterstattung (seit 2017 ist die Nachhaltigkeitsberichterstattung von kapitalmarktorientierten Unternehmen mit mehr als 500 Mitarbeitern verpflichtend; BMJV 2017) und die Unterstützung der Entwicklung von Standards der Berichterstattung Impulse der nachhaltigen Entwicklung. Corporate Social Responsibility beinhaltet das nachhaltige unternehmerische Handeln in dem Sinne, dass die Geschäftstätigkeit des Unternehmens von ökonomischen, ökologischen und sozialen Prinzipien geleitet ist (vgl. Stoll 2009: 59f., DIN 2011: 24). Oder: ,CSR bezeichnet den spezifischen Beitrag, den Unternehmen zum nachhaltigen Wirtschaften, zur Nachhaltigkeit, leisten“ (BMAS o.J.).

Es geht um die Suche nach einer Balance zwischen den drei Dimensionen gesellschaftlicher Verantwortung. Die soziale Dimension umfasst dabei gesellschaftsbezogene bzw. Gemeinwesen orientierte Prinzipien wie die Förderung der Autonomie der Menschen bei gleichzeitiger Einbettung in bzw. Teilhabe an Gemeinschaften. Sie betont die normative Grundlage und das Kernelement des Nachhaltigkeitsbegriffs, der generationen- wie Lebenswelt übergreifenden und globalen Gerechtigkeit. Es geht z. B. um Fragen nach der gerechten Verteilung von Rechten und Gütern. Dieses ist zu vereinbaren mit ökologischen Prinzipien wie einem ressourcensparenden Handeln, dem Erhalt oder der Verbesserung der Qualität von Luft, Wasser und Böden sowie dem Erhalt der Artenvielfalt. Dieses schließt einen Ressourcen schonenden Umgang mit den Lebensgrundlagen ein. Die Verfolgung der dritten Handlungsmaxime bei der Herstellung von Produkten und Dienstleistungen, der Gewinnmaximierung in der ökonomischen Dimension, sichert den jeweiligen Lebensstandard in den Gesellschaften. Eine Balance in den drei Dimensionen gesellschaftlicher Verantwortung bedeutet, dass die alleinige Berücksichtigung einer Maxime (wie z. B. des Paradigmas der Gewinnmaximierung) vor dem Hintergrund des wachsenden Bewusstseins für die Komplexität der (globalen) Wirkungszu- 
sammenhänge des (lokalen) Handelns und der Endlichkeit der uns bzw. unserem (unternehmerischen) Handeln zur Verfügung stehenden Ressourcen keine adäquate Handlungsstrategie sein kann.

Keine Organisation wird langfristig überleben können bzw. im Interesse ihrer diversen Stakeholder handeln und sich die gesellschaftliche Existenzberechtigung bewahren, wenn der Fokus entweder z. B. nur auf der eng verstandenen ökonomischen oder nur auf der eng verstandenen sozialen Dimension liegt. Soziale/ethische, ökologische und ökonomische Belange sind im konkreten Zusammenhang mit den Kernthemen der jeweiligen Unternehmen und den ihnen zugrundeliegenden Prinzipien zunächst zu identifizieren (vgl. Rusche in diesem Band). In weiteren Schritten sind zur Umsetzung gesellschaftlicher Verantwortung/der Nachhaltigkeit und der Integration von CSR in die Unternehmenstätigkeit u. a. neue, die genannten Dimensionen berücksichtigende Geschäftsmodelle zu entwickeln (vgl. Stoll in diesem Band), ebenso wie Kooperationen einzugehen (vgl. Kern, Rieger/Scholz und Herrmann in diesem Band).

\section{CSR - eine Frage von Aushandlung und Kooperation}

Vor dem Hintergrund einer gemeinsam getragenen Verantwortung steht der Begriff der Corporate Social Responsibility in einem engen Zusammenhang mit Aushandlungsprozessen zwischen unterschiedlichen gesellschaftlichen Akteursgruppen und deren Kooperation im Hinblick auf geteilte Ziele. Die Komplexität von Ursache und Wirkungszusammenhängen auf den unterschiedlichsten Ebenen (lokal, national, global) und mit ihr verbundene gesellschaftliche Herausforderungen sind offensichtlich nur noch zu erfassen, geschweige denn zu bewältigen, wenn unterschiedliche Perspektiven und Kompetenzen zusammengeführt werden (vgl. auch Schaltegger 2018: 360).

In einem Drei- bzw. Vierklang der Akteure des politisch-administrativen Systems, aus der Privatwirtschaft, der Sozialwirtschaft und dem Bereich der Zivilgesellschaft sind - in unterschiedlichen Kontexten/Rahmungen - zunächst Herausforderungen zu identifizieren, um sie dann in konkreten Zusammenhängen unter Bezug auf die unterschiedlichen Kompetenzen anzugehen (vgl. Herrmann in diesem Band).

Dieses betrifft neben innerbetrieblichen Strukturen und Prozessen insbesondere die zweite, eng mit dem Begriff Corporate Citizenship verbundene Kategorie der Corporate Social Responsibility (zur Unterscheidung vgl. Riess 2010: 589). Sie setzt das Unternehmen als „Bürger“ und Akteur in der Gesellschaft in den Fokus (vgl. Stoll 2009: 60f., Lang/Dresewski 2010: 401f., Habisch/Schwarz 2015: 114). Auch wenn diese beiden Dimensionen letztlich 
nicht wirklich zu trennen sind, interne Prozesse und Strukturen von der Außenwirkung in die Gesellschaft(en) nicht zu trennen sind, so geht es mit diesem Fokus eher darum, dass Unternehmen mit dem Ziel handeln, „konkrete Probleme ihres gesellschaftlichen Umfeldes zu lösen - etwa in sozialen oder kulturellen Projekten“ (Riess 2010: 589).

Dabei geht der Impuls der Entwicklung durchaus nicht immer von staatlicher Seite aus, agierten viele Unternehmen im Bereich der Flüchtlingshilfe (mit dem Blick auf den wachsenden Fachkräftemangel, jedoch auch mit Blick auf die ihnen zur Verfügung stehenden Ressourcen) als Treiber der Entwicklung, kam die Initiative für CSR häufig von Seiten der Unternehmen, erwies sich das gesetzliche Reglement eher als „Hemmschuh“.

\section{CSR - Eine Frage der Unternehmensgröße?}

Damit steht also jedes profit- und sozialwirtschaftliche Unternehmen - auch unabhängig welcher Größe - bewusst oder unbewusst, freiwillig oder gezwungenermaßen vor der Frage, in welchem Zusammenhang Ökonomisches, Ökologisches und Soziales in der jeweiligen Organisation steht bzw. wie der $\mathrm{Zu}-$ sammenhang gestaltet werden kann oder muss. Aus unterschiedlichen Gründen bzw. aus unterschiedlicher Perspektive begründbar, entsteht zuweilen der Eindruck „CSR“ steht maßgeblich/muss maßgeblich auf der Agenda von (internationalen) Großunternehmen oder den (Spitzen-)Verbänden der Sozialwirtschaft stehen bzw. ist nur für diese relevantes Diskussionsthema und/oder notwendiges Handlungsfeld. Jedoch stehen sowohl Großunternehmen als auch kleine und mittlere Unternehmen/Organisationen (KMU/KMO) sowohl praktisch als auch in der Diskussion mitten im gesellschaftlich verantwortlichen Denken und Handeln. Bei näherer Betrachtung scheinen vielmehr gar KMU/KMO die ,echten“ Akteure von CSR (vgl. Stoll 2009), ihre gesellschaftliche „Entfremdung“ und organisatorische Komplexität ist/scheint ,nur“ nicht derart fortgeschritten, dass nachhaltiges Handeln, wiederum über den als teilweise als abstrakt empfunden Begriff der CSR formal und abstrakt zur „Wiedereinführung" zu diskutieren wäre. Fatal wäre in diesem Zusammenhang auch die Einschätzung, dass es doch nur die ,großen Player“ sein können, die einen relevanten nachhaltigen internen oder externen gesellschaftlichen Impact und entsprechende Verantwortung haben können/müssen. Offensichtlich ist jedoch, dass Unternehmen unterschiedlicher Größe, sich z.B. hinsichtlich der organisatorischen, strukturellen Komplexität oder der sozialen Verfasstheit erheblich unterscheiden können und diese Verschiedenheit einbezogen werden muss - sowohl in der Frage, wer wie für das CSR-Leitbild steht bzw. gewonnen werden kann, wer, wie unterstützt werden kann, wer es wie in den unternehmerischen Strukturen verankert etc. (vgl. auch Stoll in diesem Band). Um nicht 
die ein oder anderen in der CSR-Debatte bzw. Umsetzung zu verlieren oder nicht für diese gewinnen zu können, muss z.B. darauf geachtet werden, dass sich sowohl Repräsentant*innen großer als auch mittlerer und kleiner Unternehmen in Diskussion und Handeln einbringen (können und möchten) und mit diesem Handeln wahrnehmbar sind. Es müssen in der CSR-Debatte z.B. unterschiedliche Art und Weisen der verbalen und praktischen Ausdrucksweise von Angelegenheiten der CSR Eingang finden, unterschiedliche Formalisierungsgrade von Handeln Beachtung finden, unterschiedlich komplexe Strukturen der Unternehmen, unterschiedliche Motivlagen und -zusammenhänge im Kontext von CSR etc. beachtet werden.

\section{CSR - Eine Frage der Entwicklung von Organisationen}

Es ist evident, dass CSR ein „Konzept“ ist, welches nicht alleine durch die Corporations/die Unternehmen realisiert werden kann bzw. dessen Verständnis und Umsetzung von der gesellschaftlichen Verfasstheit, von wirtschaftsund sozialpolitischen Rahmenbedingungen usw. abhängt, resultierend aus den diversen Aushandlungsprozessen (s.o.). Nichtsdestotrotz oder gerade deswegen ist ihre letztlich primäre „Umgebung“ das profit- oder sozialwirtschaftliche Unternehmen (oder die NGO). Entsprechend verbleibt es letzten Endes bei den Unternehmen sich unter gegebenen Bedingungen derart zu entwickeln, dass sie als gesellschaftlich verantwortliches bzw. nachhaltiges Unternehmen agieren und als solches wahrgenommen werden können.

Die CSR- und Nachhaltigkeitsdebatte hat mittlerweile diverse national und international gedachte Managementsysteme und CSR-Instrumente hervorgebracht, die Unternehmen dabei unterstützen können dem Leitbild CSR zu folgen bzw. dieses für ihre Organisation und Stakeholder stimmig zu entwickeln (s. z.B. Stoll in diesem Band). Diese zielen darauf ab, sowohl Organisationsstrukturen, -prozesse als auch Mitarbeiter*innen bzw. individuelles Verhalten derart aufzugreifen und zu entwickeln, dass das CSR-Leitbild das gesamte Unternehmen und seine Handlungen leitet. Ohne Frage ist dies eine nicht immer einfache Entwicklungsherausforderung (in nicht selten suboptimalen Rahmenbedingungen eingebettet). Dies um so mehr, da sie eine ist, die als „niemals“ abgeschlossen betrachtet werden kann und durch viele Komplexitäten geprägt ist. Diesbezüglich werden in diesem Band sowohl konkrete Managementsysteme betrachtet als auch z.B. Dilemmata-verursachende Rahmenbedingungen (s. z.B. Scholz und Rusche in diesem Band) verdeutlicht, innerhalb derer Unternehmen derzeit versuchen sich am CSR-Leitbild zu orientieren.

Im Kontext der Entwicklung einer Organisation zu einer nachhaltig gesellschaftlich verantwortlichen Organisation wird deutlich, dass CSR die normative Ebene der Unternehmen tangiert. Das Unternehmen verpflichtet sich 
(selbstbestimmt) im sozial-ökologischen-ökonomischen Zusammenhang bestimmten (i.d.R. ursprünglich ethisch-moralisch) begründeten/begründbaren Handlungsregeln (z.B. über ein Unternehmensleitbild) in entsprechenden Handlungsbereichen zu folgen. Da aber CSR als „Leitkonzept“ durchaus eine „Resultat" von den dem Unternehmen vorgelagerten moralisch-normativen Überlegungen und Entscheidungen durch deren vielfältigen Stakeholder ist, wird deutlich, dass CSR insgesamt ein Thema ist, welches unter einer ethischen bzw. schließlich auch normativen Ebene zu diskutieren und zu reflektieren ist.

Für Unternehmen birgt CSR in verschiedenster Hinsicht zudem strategische Implikationen. So kann ein Unternehmen (derzeit) durchaus überlegen, ob es über eine normative Begründung hinaus nicht strategisch (im Sinne der Existenzberechtigung/-sicherung unter den Blicken kritischer Stakeholder) klug ist, sich als gesellschaftlich verantwortlich handelndes Unternehmen aufzustellen. Zudem ist es zentral, dass die Unternehmen bezogen auf ihre interne und externe Umwelt im Auge behalten, welche der vielzähligen Handlungsbereiche im Kontext CSR, von besonderer (existenz-/erfolgsrelevanter) Bedeutung für das Unternehmen selbst und seine Stakeholder sind. Nicht zuletzt aber ist es auch von Seiten der gesellschaftlichen Institutionen (z.B. Städte, Gemeinden) strategisch klug und notwendig, die Handlungsaufforderung an Unternehmen zu richten, gesellschaftlich verantwortlich bzw. unternehmerisch nachhaltig zu agieren. Die derzeitigen gesellschaftlichen Herausforderungen, die sich in der für die Gesellschaft und ihre Systeme (Politik, Bildung, Sozialwesen, Umwelt etc.) und Institutionen stellt, evozieren nahezu eine Strategie von Städten und Kommunen, Unternehmen als gesellschaftlich verantwortlich handelnde Akteure einzubeziehen um Herausforderungen gemeinsam (nutzbringend) zu bewältigen (vgl. Herrmann in diesem Band).

Die Realisierung normativer Festlegungen und Erreichung strategischer Ziele in Unternehmen erfordert eine entsprechende operative Umsetzung. Womit sich für Unternehmen auch immer (wieder) die Frage stellt, was wie in der täglichen unternehmerischen Praxis zu tun ist, um tatsächlich auch auf eine „Umsetzungsebene“ in Sachen CSR zu kommen bzw. um die normativ gute Ausrichtung und die strategisch klugen Überlegungen zum verantwortlichen Handeln auf operativer Ebene nicht zu konterkarieren. Auch für die Stakeholder stellen sich operative Fragen unter unterschiedlicher Perspektive: Wie können z.B. Kooperationen im Sozialraum, welche (sozialwirtschaftliche und profitwirtschaftliche) Unternehmen als gesellschaftliche Akteure miteinbeziehen, konkret gestaltet werden? Was müssen, z.B. Politik, Gesetzgebung etc. tun, um die Umsetzung von CSR in den Unternehmen zu ermöglichen?

Nicht selten vermischen sich in Theorie und Praxis diese drei (normativ, strategisch, operativ) Ebenen bzw. Perspektiven. Abhängig von der konkreten praktischen und theoretischen Fragestellung unter der CSR betrachtet wird, 
kann es auch sinnvoll sein, gerade mehrperspektivisch zu denken, zu argumentieren. In manchen Kontexten (z.B. zunächst ethische Begründung dessen, was warum als sozialverantwortliches Handeln gelten kann) scheint es jedoch sinnvoll, auf der ein oder anderen Ebene zu argumentieren bzw. zumindest auf die durchaus komplexe Vermengung von Ebenen hinzuweisen. Der vorliegende Band mit den diversen Beiträgen entspricht auch in dieser Hinsicht der Komplexität von CSR. Die Beiträge beziehen sich in unterschiedlicher Weise auf eine notwendige ein- oder mehrperspektivische Betrachtung und Reflexion.

\section{Literatur}

BMAS (o.J.): Was ist CSR - Nachhaltigkeit und CSR. Internet-URL: https://www.csrin-deutschland.de/DE/Was-ist-CSR/Grundlagen/Nachhaltigkeit-und-CSR/csrgrundlagen.html [Zugriff: 20.06.2019]

BMJV (Bundesministerium der Justiz und für Verbraucherschutz) (2017): Gesetz zur Stärkung der nichtfinanziellen Berichterstattung der Unternehmen in ihren Lageund Konzernlageberichten (CSR-Richtlinie-Umsetzungsgesetz). Bundesgesetzblatt Jahrgang 2017 Teil I Nr. 20, ausgegeben zu Bonn am 18. April 2017.

DIN Deutsches Institut für Normung (2011): Leitfaden zur gesellschaftlichen Verantwortung (ISO 26000:2010). Berlin: Beuth Verlag.

Eichmann, T. (2016): Zarte Pflanze Nachhaltigkeit. In: WOHLFAHRTINTERN 2016, 5 S. $30-32$.

Europäische Kommission (2011): Mitteilungen der Kommission vom 25.10.2011: Eine neue EU-Strategie (2011-14) für die soziale Verantwortung von Unternehmen (CSR). https://eur-lex.europa.eu/legal-content/DE/TXT/PDF/?uri=CELEX:52011DC0681\&from=DE [Zugriff: 20.12.2019]

Europäische Kommission (2001): Grünbuch. Europäische Rahmenbedingungen für die soziale Verantwortung von Unternehmen. https://eur-lex.europa.eu/LexUriServ/LexUriServ.do?uri=COM:2001:0366:FIN:DE:PDF [Zugriff: 21.11.19]

Habisch, A./Schwarz, C. (2012): CSR als Investition in Human- und Sozialkapital. In: Schneider, A./Schmidpeter, R. (Hrsg.): Corporate Social Responsibility. Verantwortungsvolle Unternehmensführung in Theorie und Praxis. Berlin, Heidelberg: Springer, S. 113-133.

Hofmacher, P. (2017): Nachhaltigkeitsbericht. Das Bewusstsein schärfen. In: WOHLFAHRTINTERN 2016, 5 S. 36.

Kropp, A. (2019): Grundlagen der Nachhaltigen Entwicklung. Handlungsmöglichkeiten und Strategien zur Umsetzung. Wiesbaden: Springer Gabler.

Lang, R./Dresewski, F. (2010): Zur Entwicklung des Social Case zwischen Unternehmen und Nonprofit-Organisationen. In: Backhaus-Maul, H./Biedermann, C./Nährlich, S./Polterauer, J. (Hrsg.): Corporate Citizenship in Deutschland. Gesellschaftliches Engagement von Unternehmen. Bilanz und Perspektiven. 2. Aufl. Wiesbaden: VS Verlag für Sozialwissenschaften, S. 401-422. 
Lexikon der Nachhaltigkeit. (2016). Corporate Social Responsibility.

https://www.nachhaltigkeit.info/artikel/corporate_social_responsibility_unternehmerische 1499.htm. [Zugriff: 02.122019]

Richtlinie $20 \overline{1} 4|95| \mathrm{EU}$ des Europäischen Parlaments und des Rates vom 22. Oktober 2014 zur Änderung der Richtlinie 2013|34|EU im Hinblick auf die Angabe nichtfinanzieller und die Diversität betreffender Informationen durch bestimmte Unternehmen und Gruppe (ABl. vom 15.11.2014, L330/1-L330/9).

Riess, B. (2010): Unternehmensengagement - ein Beitrag zur gesellschaftlichen Selbststeuerung zwischen Markt und Staat. In: Backhaus-Maul, H./ Biedermann, C./Nährlich, S. [u.a.] (Hrsg.): Corporate Citizenship in Deutschland. Gesellschaftliches Engagement von Unternehmen. Bilanz und Perspektiven. 2. Aufl. Wiesbaden: VS Verlag für Sozialwissenschaften, S. 588-600.

Schaltegger, S. (2018): Unternehmerische Verantwortungsübernahme für gesellschaftlichen Nutzen. In: Backhaus-Maul, H./Kunze, M./Nährlich, S. (Hrsg.): Gesellschaftliche Verantwortung von Unternehmen in Deutschland. Wiesbaden: Springer VS, S. 351-364.

Stoll, B. (2009): Sozial und ökonomisch handeln. Corporate Social Responsibility kleiner und mittlerer Unternehmen. Frankfurt/New York: Campus Verlag. 


\title{
Gesellschaftlichen Herausforderungen mit Sozialen Kooperationen begegnen
}

\author{
Heike Herrmann
}

\section{Einleitung: Sich wandelnde „Verhältnisse“}

Ausgangspunkt der folgenden Überlegungen ist eine festzustellende veränderte Rollen- und Aufgabenverteilung zwischen Staat, Wirtschaft und Zivilgesellschaft, die sowohl mit einem neuen Selbstverständnis der Akteure der genannten Sektoren als auch mit neuen Beziehungen zwischen den Akteuren einhergeht. Bereits im Zuge des am Ende der 1980er Jahre und in den 1990er Jahren in der öffentlichen Verwaltung umgesetzten Neuen Steuerungsmodells (New Public Management) entwickelten sich neue Beziehungen zwischen staatlicher Verwaltung und der Privatwirtschaft (z. B. in Form von Public-Privat-Partnership) ebenso wie veränderte Auftragsbeziehungen und Steuerungselemente zwischen öffentlicher Hand und sozialwirtschaftlichen Unternehmen (vgl. Herrmann 2001; Oschmiansky 2010; Rasche/Morsing/Moon 2017: Part IV; Herrmann 2019: 49ff). Es existiert

„einerseits ein relativer Bedeutungsverlust sozialstaatlicher Verantwortung in ihrer herkömmlichen Form, verbunden mit einem Zwang oder Anreiz, entsprechende Lasten und Verantwortungen auf bürgerschaftliche und marktförmige Elemente zu übertragen; andererseits ein Kompetenzzuwachs für nicht-staatliche Akteure, der von ihnen selbst auch als Aufwertung erlebt wird und oft mit einer veränderten Definition öffentlicher Verantwortung und entsprechender sozialer Dienste, Einrichtungen und Qualitäten einhergeht" (Evers 2002: 217; vgl. auch Braun 2008: 14; Hofmann/Dunschen 2017: 194).

Diese von Evers angedeutete Verschiebung von sozialstaatlicher Verantwortung in Richtung privatwirtschaftlicher und zivilgesellschaftlicher Akteure ist in vielerlei Hinsicht problematisch, da die latente Gefahr der Umsetzung von 
staatlichen Sparmaßnahmen zu Lasten der Bürger einschließlich der privatwirtschaftlichen Unternehmen als Corporate Citizen ${ }^{1}$ besteht (vgl. Kleine-König 2018: 311f). Gleichzeitig haben gesellschaftliche Herausforderungen zu Beginn des 21. Jahrhunderts ein Bewusstsein dafür entstehen lassen, dass Nationalstaaten allein mit der Regulierung und Krisenbewältigung aktueller Entwicklungen und ihrer Folgen überfordert sind: Die sogenannte Finanzkrise, u. a. verbunden mit Folgen auf den lokalen Immobilien- und Wohnungsmärkten, weltweite Migrations- bzw. Fluchtbewegungen und nicht zuletzt spürbare Folgen des Klimawandels fordern auch in den Kommunen dazu auf, in sektorenübergreifenden Netzwerken und Partnerschaften nach neuen Handlungsansätzen sowie neuen Formen der Steuerung zu suchen.

Das diesem Beitrag zugrundeliegende Verständnis einer gesellschaftlichen Verantwortung im Sinne einer Corporate Social Responsibility (CSR) kann nur dann tragfähig umgesetzt werden, wenn neben Akteuren des Staates, profit- wie sozialwirtschaftliche Unternehmen und zivilgesellschaftliche Akteure im Einzelnen und gemeinsam Verantwortung im Sinne einer ökonomischen, ökologischen und sozialen Verantwortung übernehmen. Jedoch folgen politisch-administrative, privatwirtschaftliche Akteure, Unternehmen der Sozialwirtschaft ${ }^{2}$ (nachstehend auch synonym als soziale Organisationen/sozialwirtschaftliche Unternehmen bezeichnet) und zivilgesellschaftliche Akteure unterschiedlichen Interessen und Handlungslogiken, agieren in unterschiedlichen Zeithorizonten und auf unterschiedlichen Märkten. So braucht beispielsweise ein Zusammenwirken profitwirtschaftlicher und sozialwirtschaftlicher Unternehmen in sogenannten Sozialen Kooperationen spezifische Aushandlungsprozesse $^{3}$ zwischen den Akteuren, die sich zudem auf ausgewählte Zielebenen und Bereiche beziehen.

Der vorliegende Beitrag befasst sich zunächst mit der jüngsten Entwicklung einer Zusammenarbeit zwischen profitwirtschaftlichen und sozialwirtschaftlichen Unternehmen im Zuge der Übernahme von gesellschaftlicher Verantwortung. Vorgestellt werden $u$. a. erfolgversprechende und erfolghinderliche Faktoren einer Kooperation zwischen diesen unterschiedlichen Welten. Im Anschluss verdeutlicht ein Beispiel, welche spezifischen Faktoren aus der Sicht eines profitwirtschaftlichen Unternehmens in einem spezifischen Handlungsfeld des unternehmerischen gesellschaftlichen Engagements (der Bewältigung der Herausforderung der Förderung von Teilhabe und Integration von

1 Zu diesem Begriff vgl. Stoll 2009; Lang/Dresewski 2010; Habisch/Schwarz 2015: 114.

2 Dabei umfasst die Begrifflichkeit „Sozialwirtschaft“ im Folgenden in einem engen Sinn die „Produzenten sozialer und gesundheitsbezogener Dienstleistungen“ (Grunwald 2014, S. 36). Sozialwirtschaftliche Organisationen werden entsprechend als Institutionen definiert, die fremde Bedarfe decken bzw. die soziale Dienstleistungen erbringen (vgl. Arnold/Maelicke 2014, S. 11).

$3 \mathrm{Zu}$ den Aushandlungsprozessen zwischen profitwirtschaftlichen Unternehmen mit dem Ziel einer collective action siehe Riegler und Scholz in diesem Band. 
geflüchteten Menschen) in der Vergangenheit das Bemühen um Verantwortungsübernahme beeinflussten. Es geht hierbei vor allem um die Schnittstellen zwischen kommunalem und unternehmerischen Handeln. Hieran anknüpfend stellt sich die Frage, welche Rolle die Sozialwirtschaft in Bezug auf eine gemeinsame Bewältigung der dargestellten Herausforderung übernehmen könnte.

\section{Vom gesellschaftlichen Engagement zu Sozialen Kooperationen}

Unternehmerisches Engagement hat eine lange Tradition. Einige Verpflichtungen, so zur Einhaltung arbeits-, sozial- und umweltrechtlicher Regelungen oder die Einhaltung von Tarifverträgen, sind im deutschen Sozialstaatsmodell gesetzlich verankert (vgl. Braun 2008: 6). Darüber hinaus reichendes freiwilliges unternehmerisches Engagement, dies zeigen die Engagementberichte der Bundesregierung, ist zu einem hohen Prozentsatz (2012 fast 90 Prozent; vgl. Kleine-König 2016: 29) standortbezogen; d.h. standortbezogenes Engagement ist die Regel (vgl. Braun 2010: 7). Die Unternehmen unterstützen lokale Vereine oder auch Feste an ihren Unternehmensstandorten, engagieren sich in den Einrichtungen der Kinder- und Jugendhilfe oder in Kitas.

Umfang, Formen und das zugrundeliegende Verständnis des freiwilligen Engagements von profitwirtschaftlichen Unternehmen haben sich sicherlich nicht zuletzt aufgrund der mit der „CSR-Richtlinie“ (Richtlinie 2014/95/EU) angestoßenen Entwicklung im Verlauf der letzten Jahre verändert. Sebastian Braun gibt auf der Grundlage einer im Herbst 2006 durchgeführten Befragung von Profit-Unternehmen mit einem Jahresumsatz von mindestens einer Millionen Euro und mindestens zehn Mitarbeiter*innen einen Überblick über den Umfang, Struktur und Differenzierung des freiwilligen gesellschaftlichen Engagements von Unternehmen. Formen des Engagements bestehen laut Braun zu diesem Zeitpunkt überwiegend aus den klassischen Formen der Geld- und Sachspenden (vgl. Braun 2008: 9). ${ }^{4}$ Als innovatives Instrument bezeichnete er die betriebliche Unterstützung des ehrenamtlichen Engagements der Beschäftigten (Corporate Volunteering), welches mit 60\% ebenfalls weit verbreitet war. Auch er stellte fest, dass die Auswahl der Maßnahmen und Projekte sich überwiegend an dem direkten gesellschaftlichen Umfeld des Unternehmens orientierte, wobei von Seiten der Unternehmen darauf geachtet wurde, dass die Anfragen thematisch zum Unternehmen passen. Die Handlungsfelder des Sports und der Freizeit waren dabei am interessantesten; erst mit deutlichem

Sponsoring wurde als klassisches Instrument im Marketing-Mix bei der Erhebung nicht berücksichtigt. 
Abstand zeigte die Untersuchung Engagement in den Bereichen „Erziehung und Bildung“, „Kommune und Gemeinwesen“ und „Soziales“ auf (vgl. Braun 2008: 10). Die Reihenfolge der zu 60\% von den Unternehmen gesuchten Kooperationspartner*innen entsprach dieser Schwerpunktsetzung, wenn 70\% der Unternehmen das Vereinswesen unterstützten, Bildungseinrichtungen, Wohlfahrtsverbände oder Kommunalverwaltungen erst mit deutlichem Abstand folgten (ebd.). Braun vermutete, dass die Unternehmen sich insbesondere in Bereichen engagieren, die durch staatliches Handeln weniger berührt und aufgrund dessen wenig reglementiert sind. Dieses ist in den Bereichen Freizeit und Sport am ehesten der Fall. Auch sind die Aktivitäten in diesen Bereichen vergleichsweise öffentlichkeitswirksam zu gestalten.

Fragen wir nach der zugrundliegenden Handlungslogik des Engagements, so tritt nach seinen Ergebnissen die typische Verwertungslogik der profitorientierten Unternehmen einschließlich des Strebens nach Effektivität und Effizienz in den Hintergrund. Dies bedeutet allerdings auch, dass das Engagement bei weniger als einem Drittel der Unternehmen Element der aktuellen Geschäftsstrategie war (vgl. Braun 2008: 11). Stattdessen war das Engagement stark personalisiert: Führungskräfte oder hierfür eingerichtete Stellen der Öffentlichkeitsarbeit engagierten sich in Themenfeldern, die ihnen persönlich nahelagen. ${ }^{5}$ Ein weiteres grundsätzliches Ergebnis der Untersuchung war die Zweiteilung des gesellschaftlichen Engagements der Unternehmen in das der kleineren und mittleren Betriebe, die vor allem lokales, zivilgesellschaftlich eingebettetes Engagement zeigten und die Großunternehmen, die sich an der internationalen Debatte um gesellschaftliches Engagement orientierten und, so Braun, tendenziell eine Integration in ihr unternehmensinternes Selbstverständnis anstrebten (vgl. ebd.: 12; vgl. die Beiträge von Stoll sowie Stoll/ Herrmann in diesem Band).

In einer Studie der Bertelsmann Stiftung aus dem Jahr 2015 - etwa 10 Jahre später - stellten die Autor*innen fest, dass die Bedeutung des CSR-Gedankens (nach Einschätzung von Führungskräften von Großunternehmen) in den letzten Jahren zugenommen hat. Die sowohl auf der Bundesebene zunächst in Form des „CSR-Aktionsplans“ (BMAS 2010), vor allem jedoch auf der europäischen Ebene in Form der sogenannten „CSR-Richtlinie“ (Rn. 6: Richtlinie 2014/95/EU) formulierte Aufforderung zur Verantwortungsübernahme konkretisierte sich unternehmensintern, d.h. in Bezug auf die Beschäftigten, die in der Wertschöpfungskette oder in Dienstleistungen für Unternehmungen tätig sind. Jedoch auch in sozialen und kulturellen Projekten, die durch unternehmerisches Engagement unterstützt oder auf den Weg gebracht werden (vgl. Riess 2010: 589). Der Blickwinkel, so Hollmann und Schlicher, habe sich seit dem Jahr $2005^{6}$

5 Dies entspricht den Ergebnissen der weiter unten dargestellten eigenen Untersuchung aus dem Jahr 2017.

6 Die Autoren führten wie Braun im Jahr 2005 eine erste Befragung durch. 
„spürbar erweitert, was sich im Umfang der als relevant betrachteten Akteure und Interessen widerspiegelt, sei es als Verantwortung gegenüber der Umwelt, der eigenen Region oder den Zulieferbetrieben. [...] Während die Motivation für sozial-verantwortliches Unternehmertum 2005 noch primär innerbetrieblich begründet wurde (motivierte Mitarbeiter, Weiterführung der Unternehmenstradition), wird CSR heute stärker als imageförderndes Instrument begriffen, das sich sowohl positiv auf die Reputation als auch die Attraktivität des Unternehmens als Arbeitgeber auswirkt" (Hollmann/Schlicher 2015: 4).

Im Vergleich zu der von Braun durchgeführten Befragung hat der Engagementbereich der „Bildung“ in der von Hollmann und Schlichter durchgeführten Befragung deutlich an Bedeutung zugenommen: „Bildungsprojekte stehen hoch im Kurs" (Hollmann/Schlicher 2015: 8). Dabei zielten die Aktivitäten weiterhin primär auf das Unternehmensumfeld. Die Autoren stellten jedoch fest, dass dies vor allem bei Unternehmen in Produktionszweigen mit starkem Regionalbezug wie z. B. Dienstleistungsunternehmen der Fall war. Das Verständnis der gesellschaftlichen Verantwortung von Seiten der Unternehmen sei hier stark lokal verankert (vgl. Hollmann/Schlicher 2015: 7). Vertreter*innen der exportorientierten Branchen betonten dagegen die globale Verantwortung.

Kooperationspartner*innen der Profitwirtschaft waren in der Vergangenheit vor allem andere profitwirtschaftliche Unternehmen. Heute, so Hollmann und Schlicher, setzen Großunternehmen in Deutschland deutlich häufiger (in acht von zehn Unternehmen) auf die Zusammenarbeit mit gemeinnützigen oder karitativen Organisationen.

Tabelle 1: Kooperationen im Rahmen des CSR-Engagements

\begin{tabular}{|l|c|c|c|}
\hline & $\begin{array}{l}\text { Prozent der } \\
\text { Befragten } \\
\text { im Jahr 2015 }\end{array}$ & $\begin{array}{l}\text { Prozent der } \\
\text { Befragten } \\
\text { im Jahr 2005 }\end{array}$ & Veränderung \\
\hline $\begin{array}{l}\text { Gemeinnützige oder karitative } \\
\text { Organisationen }\end{array}$ & 81 & 54 & +27 \\
\hline $\begin{array}{l}\text { Unternehmensverbände, andere } \\
\text { Unternehmen }\end{array}$ & 63 & 56 & +7 \\
\hline Wissenschaft, Hochschulen & 62 & 42 & +20 \\
\hline Politik, Verwaltung & 47 & 34 & +13 \\
\hline Bürgergruppen & 36 & 16 & +20 \\
\hline Umweltorganisationen & 26 & 12 & +14 \\
\hline $\begin{array}{l}\text { NGOs, z. B. Menschenrechts- } \\
\text { organisationen }\end{array}$ & 22 & 6 & +16 \\
\hline
\end{tabular}

Quelle: Hollmann/Schlicher 2015: 11

Darüber hinaus, so auch das Ergebnis einer Untersuchung des BBSR zum Engagement von profitorientierten Unternehmen in der Quartiersentwicklung aus dem Jahr 2015, nehmen die Kommunen im gesellschaftlichen und räumlichen Umfeld des Unternehmens eine immer zentralere Vermittlungsrolle ein (vgl. 
BBSR 2015). Zwei Drittel der von Hollmann und Schlicher befragten Führungskräfte halten es für zutreffend, ,dass die Problemlagen der Kommunen zunehmend regionales und lokales Engagement der Betriebe erfordern, etwa jeder Fünfte vertritt diese Ansicht gar in besonderem Maße" (Hollmann/Schlicher 2015: 7). Dabei kommt den Kommunen die Aufgabe zu, Handlungsfelder und Bedarf aufzuzeigen. Damit sind in Bezug auf das oben als bedeutsam eingestufte lokale Engagement zwei durchaus wiederum sehr unterschiedliche zentrale Partner*innen der gemeinsamen Verantwortungsübernahme genannt: Soziale Organisationen und Kommunen. Auch Wissenschaftler*innen und verschiedene Bürgergruppen, d. h. die Zivilgesellschaft, nehmen zunehmend eine wichtigere Rolle als Kooperationspartner*innen ein (vgl. Tab. 1).

Neben (Projekt-) Partnerschaften in einem festgelegten zeitlichen Rahmen entstanden dauerhafte Soziale Kooperationen zwischen den sehr unterschiedlichen Handlungslogiken folgenden und sich auf sehr unterschiedlichen Märkten bewegenden Akteuren. Die Sozialen Kooperationen gehen über ein punktuelles Engagement hinaus. Knapp zwei Drittel der Befragten betonten die langfristige Ausrichtung ihres Engagements (vgl. Hollmann/Schlicher 2015: 9).

„'Soziale Kooperationen' sind solche neuen Partnerschaften von engagierten Unternehmen, gemeinnützigen Organisationen und öffentlichen Verwaltungen, in denen sich Unternehmen über das traditionelle Spenden und Sponsern finanzieller Mittel hinaus mit vielfältigen Ressourcen und ihren spezifischen Kompetenzen in die Gestaltung ihres Umfeldes einbringen, um damit für sich, ihre gemeinnützigen Kooperationspartner, deren Adressaten und das Gemeinwesen/den Standort insgesamt einen Nutzen zu erzielen" (Lang/Kromminga/Dresewski 2007: 1),

so formulierten es Lang, Kromminga und Dresewski aus der Sicht der profitorientierten Unternehmen.

„Bei Unternehmenskooperationen geht es also weder ,nur’ um Wohltätigkeit, noch allein um die (ergänzende) Unterstützung von Trägern oder sozialarbeiterischen Initiativen mit Geld gegen Werbeleistung. Vielmehr geht es um Partnerschaften mit Firmen, die mit ihrem Engagement dazu beitragen können, dass Gelegenheiten und Strukturen geschaffen und/oder gefestigt werden, die für die Belange der verschiedenen Gruppen und ihr Miteinander im Gemeinwesen förderlich sind“ (Damm/Lang 2002: 19f).

Und weiter stellen die Autoren fest: „Kooperation basiert auf Kontakten zwischen einander, fremden Welten' sozial und ökonomisch tätiger Mitglieder des Gemeinwesens, die über bislang starren Grenzen von Markt, Staat und drittem Sektor hinausgehen" (ebd.). 


\section{Hindernisse und Erfolgsfaktoren Sozialer Kooperationen}

Hollmann und Schlicher nennen folgende Hindernisse gesellschaftlichen Engagements von Großunternehmen (vgl. Hollmann/Schlicher 2015: 14):

- knappe finanzielle Ressourcen

- fehlende Messbarkeit der Wirkung

- keine entsprechende Unternehmenskultur oder -werte

- fehlender Nutzen für das Unternehmen

- fehlende Kenntnisse über die Umsetzung von CSR-Projekten

- fehlende Kenntnisse über sinnvolle Themen

- fehlende Ansprechpartner*innen bei der Umsetzung

- fehlende gesellschaftliche Anerkennung

Letztlich ist auch die Tatsache, dass Kooperationsprojekte häufig zufällig und personenbezogen entstehen (vgl. BBSR 2015: 5) - weil gerade die richtigen Personen zur richtigen Zeit am richtigen Ort zusammengetroffen sind - ein Hindernis einer kontinuierlichen Sozialen Kooperation. Erst das Einfließen der Ziele des Engagements und der Strategien ihrer Verfolgung in die Corporate Identity der beteiligten Unternehmen lassen das Engagement und die Kooperation dauerhaft tragfähig werden.

In einer im Jahr 2017 durchgeführten Befragung von profitwirtschaftlichen Unternehmen wurden als hinderliche Bedingungen der Kooperation mit Unternehmen der Sozialwirtschaft die fehlende Augenhöhe in der Kooperation, fehlende Beharrlichkeit, Personalfluktuation, aber auch unterschiedliche Wertvorstellungen und unzureichende inhaltliche Auseinandersetzung mit der Kooperation genannt (vgl. Stoll/Herrmann 2018). In der fehlenden Augenhöhe drückt sich eine Differenz in der gesellschaftlichen Anerkennung der Handlungsprinzipien profit- und sozialwirtschaftlicher Unternehmen aus. Aus Sicht der profitwirtschaftlichen Unternehmen fällt es den sozialen Organisationen schwer, das Gegenüber nicht als „Gegner“ zu sehen. Während in den Äußerungen der Profit-Unternehmen zum Ausdruck kommt, dass akzeptiert wird, dass Handlungsmaximen wie die Gewinnorientierung auf der einen Seite und Gemeinwohlorientierung auf der anderen Seite auch Trennendes erzeugen, werden bei den Sozialunternehmen Probleme in der Akzeptanz von Unterschieden gesehen. Auch haben die Profit-Unternehmen den Eindruck, es ist den Sozialunternehmen unangenehm, die Kooperation als Nutzen und Gewinn bringend zu definieren (,,...und da ist es auch okay, wenn beide ihren Nutzen draus ziehen"; I7, S. 102). Die Handlungsmaximen der Gewinn- und Gemeinwohlorientierung, so die Erfahrung der Profit-Unternehmen, erscheinen nach wie vor unvereinbar.

Mit den Buchstaben sind die jeweiligen Interviews der Befragung bezeichnet. 
Dass Vieles in der Kooperation von den in diesem Feld agierenden Personen abhängt, wird anhand der Probleme mit der Personalfluktuation und einer fehlenden Beharrlichkeit deutlich. Es zeigt sich, dass selbst bei Unternehmen mit starkem Engagement die Strukturen einen Wechsel von Personal kaum auffangen. Am schwersten wiegen jedoch die Aussagen zur unzureichenden inhaltlichen Auseinandersetzung mit der Kooperation in den sozialwirtschaftlichen Unternehmen. Dies weist auf die Notwendigkeit von kontinuierlichen Aushandlungsprozessen bei sich wandelnden gesellschaftlichen (etwa auch gesetzlichen) Rahmenbedingungen hin. Es ist nicht ausreichend, sich lediglich bei der Gründung der Partnerschaft mit den Handlungsbedingungen und Vorstellungen des Gegenübers auseinander zu setzen; wobei auch im Prozess des Herantretens an die Profit-Unternehmen mangelnde Bereitschaft festgestellt wurde, sich über das Gegenüber zu informieren. Es braucht „,eine ausführliche Kennlernphase, wo beide auch ihre Ziele, warum macht man das überhaupt, sorgfältig abklopfen und dann aber auch ggf. das Ganze in eine Art Rahmenvertrag gießen“ (B, S. 8) und eine kontinuierliche Weiterführung innerhalb der Kooperation. Die Quintessenz ist wie so oft: Der Aufbau und die Pflege einer Kooperation braucht Zeit in Form von Personaleinsatz, die grundsätzliche Bereitschaft der Beteiligten und neben den inhaltlichen Kompetenzen auch solche in der Kommunikation und in Aushandlungsprozessen mit einem (anderen Handlungsmaximen folgenden) Gegenüber.

Als förderliche Bedingungen der Sozialen Kooperation wurden von den Profit-Unternehmen ein motivations- und fähigkeitsbezogener Einsatz beider Kooperationspartner*innen, eine realistische Einschätzung über die Sinnhaftigkeit, eine hohe Transparenz und Offenheit (die durch persönliche Sympathie und gegenseitiges Vertrauen gefördert wird) genannt.

\begin{abstract}
„,... der Schlüssel zu einer guten Kooperation ist eine gute Kommunikation. Kommunikation im Sinne von... mit dem Partner ins Gespräch kommen, rechtzeitig die Einrichtung besuchen, immer wieder Kontakt pflegen, also denen auch mal unsere Unternehmenswelt vorstellen, in unsere eigene Welt eintauchen“" (G, S. 93).
\end{abstract}

Dahinter steht auch die Notwendigkeit einer beiderseits passenden Auswahl des Engagementfeldes, welches den beiderseitiger Einsatz von Kompetenzen erlaubt.

Soziale Kooperationen sind Anlass der Reflexion der eigenen Arbeit. Die eigenen Inhalte und Zielsetzungen werden klarer formuliert und hinterfragt, um sie in die Kommunikation einbringen zu können. Der im Zuge der Kooperation stattfindende Austausch kann zudem Impulse in Bezug auf eingeschliffene Arbeitsabläufe setzen. Gleichzeitig erleben die Partner eine gegenseitige Wertschätzung ihrer Arbeit und ihres Engagements. Gleiches gilt im Übrigen auch für die Zielgruppen des Engagements. Soziale Kooperationen bieten, für beide Seiten der Kooperation, die Chance einer Organisationsentwicklung, die den Aufbau von für die Vorhaben tragfähigen Netzwerken und die Erweiterung von Kompetenzen beinhaltet. 
Forschungsbedarf besteht zum jetzigen Zeitpunkt in Bezug auf hinderliche und förderliche Bedingungen der Sozialen Kooperation von Seiten der Sozialen Organisationen. Welche Arbeitsfelder sehen sie als besonders geeignet an, um eine für beide Seiten gewinnbringende Kooperation mit profitwirtschaftlichen Unternehmen einzugehen? Die sozialraumorientierte Soziale Arbeit bot und bietet sicherlich auch in Zukunft dabei einen Schwerpunkt der Zusammenarbeit. Zielgruppenspezifisch ist die Ausbildung Jugendlicher und junger Erwachsener ein weiterer Schwerpunkt Sozialer Kooperationen. Aus diesem Bereich stammt das im folgenden Abschnitt genannte Beispiel aus der Praxis eines Profit-Unternehmens, das sich im Rahmen der Flüchtlingssozialarbeit engagierte. Die folgenden Überlegungen nehmen die Darstellung eines profitwirtschaftlichen Unternehmens zum Anlass, die Rolle der Kommunen in der gemeinsamen Übernahme gesellschaftlicher Verantwortung zu beleuchten. Es wird deutlich, dass es vor allem am Zusammenspiel der einleitend genannten Akteure des Staates, profit- wie sozialwirtschaftlicher Unternehmen und zivilgesellschaftlicher Akteure in Bezug auf eine gemeinsam getragene gesellschaftliche Verantwortung fehlt.

\section{Die Rolle der Kommunen im Hinblick auf gesellschaftliches Engagement von Profit-Unternehmen - ein Beispiel}

Das unternehmerische Engagement steht im folgenden Beispiel in engem $\mathrm{Zu}$ sammenhang mit den gesellschaftlichen Herausforderungen, die Mitte der 2010er Jahre aufgrund der angestiegenen Anzahl von geflüchteten Menschen in Deutschland entstanden sind. Für die Soziale Arbeit bzw. die Sozialen Organisationen entstanden insbesondere im Feld der Flüchtlingssozialarbeit und der sozialraumorientierten Sozialen Arbeit, aber auch in weiteren Arbeitsfeldern wie der Schulsozialarbeit neue Herausforderungen (vgl. Herrmann 2019). Insgesamt ist der Weg der Geflüchteten von der Ankunft in Deutschland bis zur zumindest partiellen Integration der Menschen von einer Vielzahl von strukturellen und individuellen Hindernissen geprägt. Dies gilt aus Sicht der profitwirtschaftlichen Unternehmen auch und vor allem in Bezug auf die Integration in die Ausbildung und den Arbeitsmarkt, auf die sich die folgenden Aussagen konzentrieren. Ging es zunächst um eine erste Phase der Aufnahme von Geflüchteten und die Gewährleistung von Schutz und Sicherheit im unmittelbaren Umfeld, standen und stehen insbesondere die Kommunen anschließend vor der Aufgabe, über eine Eingliederung in das Bildungssystem und die Gewährleistung von Teilhabe am Arbeitsleben eine langfristige Integrationsperspektive für anerkannte Asylbewerber*innen zu eröffnen. 
Wie dieses Thema die Arbeit der Kommunen beeinflusste, zeigen die Ergebnisse einer Befragung von 30 Kommunen, die im Rahmen der vom BBSR in Auftrag gegebenen Untersuchung zur Integration von Zuwanderern - Herausforderungen für die Stadtentwicklung durchgeführt wurde. Es wurden folgende (hier in der Anzahl der Nennungen in absteigender Reihenfolge aufgeführten) Herausforderungen genannt (vgl. empirica ag 2017: 4):

- Wohnraumversorgung von Zuwanderern

- Mangelnde individuelle Qualifikationen für den Arbeitsmarkt

- Zuwanderung von Schutz/Bleibeberechtigten

- Organisatorische/formale Hemmnisse Arbeitsmarkt

- Unzureichende Teilhabe am gesellschaftlichen Leben

- Zunahme von Fehlbelegern

- Einrichtungsbezogene Segregation in den Schulen

- Kapazitätsengpässe Kita-Plätze

- Erreichbarkeit/Beteiligung von Personengruppen

- Wachsender Sozialneid

- Zunahme Unsicherheitsempfinden Bestandsbevölkerung

- Organisation von Ehrenamt

- Kapazitätsengpässe Sprachangebote

- Kapazitätsengpässe bei individueller Begleitung/Beratung

- Einrichtungsbezogene Segregation in Kitas

- Zunahme der Konzentration in Quartieren

- Kapazitätsengpässe Angebote beruflicher Qualifikation

Die genannten Punkte benennen Bereiche der Steuerung und Bereitstellung der notwendigen Infrastruktur in der (Aus-)Bildung. Neben der Wohnraumversorgung der Zuwanderer ist es vor allem die mangelnde Qualifikation für den (lokalen) Arbeitsmarkt, die die Kommunen in ihrer Aufgabenerfüllung beschäftigt. Auch werfen Kapazitätsengpässe, z. B. im Hinblick auf Sprachangebote und Angebote beruflicher Qualifikation, Probleme auf. Der hier festgestellte hohe Bedarf wird auch von Hofmann und Dunschen gesehen, die feststellen: „Mit dem im Mai 2016 verabschiedeten Integrationsgesetz hat die Bundesregierung wichtige Rahmenbedingungen geschaffen, um die Einstellung von Geflüchteten in Unternehmen zu erleichtern. Dennoch bleiben Sprachbarrieren und teilweise mangelnde oder schwer nachweisbare Qualifizierung eine Herausforderung für die Wirtschaft, der sich gezielt zu stellen gilt"“ (Hofmann/Dunschen 2017: 200). Dies um so mehr, da Erwerbstätigkeit erwiesenermaßen eine zentrale Rolle im Hinblick auf soziale Teilhabe und Integration (auch) von geflüchteten Menschen spielt. Sie vermittelt Anerkennung, erhöht das Selbstwertgefühl und bietet die Möglichkeit, soziale Netzwerke und damit soziales Kapital aufzubauen (vgl. Weingarten/Wohlert 2018).

Von Seiten eines befragten Unternehmensvertreters eines profitwirtschaftlichen Unternehmens im Nachgang der bereits genannten Befragung aus dem 
Jahr 2017 (vgl. Stoll/Herrmann 2018) wurden neben dem Verfahren der gesetzlichen Anerkennung des Asylstatus vor allem folgende Schwierigkeiten genannt:

- Spracherwerb organisieren,

- bei der Wohnungssuche unterstützen,

- Gestaltung des Mietverhältnisses (Stromanbieter auswählen und Verträge unterschreiben, später Nebenkosten-Rechnungen verstehen usw.),

- Eingliederung ins Gesundheitssystem (Krankenkassenanmeldung),

- Eingliederung in Bildungs- und Betreuungssysteme in Bezug auf die Kinder und

- eine generell notwendige Unterstützung bei Behördengängen.

Die von den Kommunen und dem Vertreter des Profit-Unternehmens genannten Herausforderungen bzw. Schwierigkeiten sind in Teilen identisch. Der Unternehmensvertreter beschreibt die wahrgenommene Orientierungslosigkeit der Neuankömmlinge, die aufgrund nicht vorhandener Sozialisation innerhalb der Bundesrepublik viele Schritte der Integration in die Systeme nachholen müssten; so beispielsweise Impfungen, die erst den Schulbesuch der Kinder ermöglichen. „Lost in systems“ ist der Begriff, den er in diesem Zusammenhang wählte. Dabei können die Geflüchteten nicht auf verwandtschaftliche Unterstützungsnetzwerke oder bestehende soziale Netze, d. h. Formen des sozialen Kapitals von Alteingesessenen aufbauen, sondern sind auf die Unterstützung durch die öffentliche Hand (z. B. durch beauftragte Unternehmen der Sozialwirtschaft) oder - so wird es insbesondere für die erste Zeit nach der Ankunft beschrieben - durch zivilgesellschaftliches Engagement von Bürger*innen angewiesen. Hier wurden in der Schilderung des Unternehmensvertreters erste Grenzen des Engagements des profitwirtschaftlichen Unternehmens ebenso wie des zivilgesellschaftlichen Engagements deutlich. Es wurden konkrete Handlungsfelder benannt, in denen die grundsätzliche Verantwortung des Staates, trotz der gesellschaftlichen Verantwortung von privatwirtschaftlichen Unternehmen, nicht auf nicht-staatliche Akteure übertragen werden kann. Gleichzeitig existieren genau in diesen Bereichen (Aufbau von Unterstützungsnetzwerken und sozialen Netzen, Orientierungshilfen in den Systemen) Kompetenzen der Sozialwirtschaft.

Privatwirtschaftliche Unternehmen engagierten sich - nicht zuletzt auch aus betriebswirtschaftlichen Interessen wie einem Ausgleich in Bezug auf den Fachkräftemangel in bestimmten Branchen - im Hinblick auf eine Teilhabe Geflüchteter am Ausbildungssystem bzw. den Arbeitsmarkt. Erste Kontakte zu den Betrieben entstanden im Rahmen von Praktika und Einstiegsqualifizierungen. ${ }^{8}$ Hier können nach ersten Erfahrungen in privatwirtschaftlichen Unternehund dem TÜV Rheinland. 
men Mentorenprogramme, eigens in den Betrieben durchgeführte Sprachkurse, aber auch in interkulturellen Trainings geübte Fähigkeiten wie Pünktlichkeit, Verlässlichkeit, ein tolerantes Miteinander, eine Feedbackkultur und das Arbeiten in Hierarchien vermittelt werden (vgl. auch Hofmann/Dunschen 2017: 201).

Ein weiterer zentraler Schritt im Rahmen des Integrationsprozesses ist aus Sicht des befragten Profit-Unternehmens der Vermittlungsprozess von Ausbildungs- und Arbeitsplätzen. In sozialen Kooperationen zwischen sozialwirtschaftlichen Unternehmen (die auf eine langjährige Erfahrung der Vermittlung in das Ausbildungssystem und in den Arbeitsmarkt für Menschen in sozialen Problemlagen zurückgreifen können) und privatwirtschaftlichen Unternehmen (die entsprechende Ausbildungs- und Arbeitsplätze zur Verfügung stellen und ebenfalls über Erfahrungen im Ausbildungs- und Beschäftigungsbereich verfügen) mussten und müssen neue Wege gefunden werden, um das Ziel der Arbeitsvermittlung zu erreichen. So schreiben auch Hofmann und Dunschen:

„Während für das kurz- und mittelfristige Engagement für Geflüchtete weitgehend auf Erfahrungswerte aus andere Projekten zurückgegriffen werden konnte, erforderten die nachhaltige Schaffung von Ausbildungsplätzen, die Gewinnung von geeigneten und motivierten Auszubildenden sowie die Etablierung eines Integrationsprogrammes Pioniergeist" (Hofmann/Dunschen 2017: 202).

Die Kommunen bzw. Ämter agierten nach der Erfahrung des Unternehmensvertreters dabei häufig parallel, statt in Kooperationen. Sowohl von Seiten der dem Beispiel zugrunde gelegten Befragung des Unternehmensvertreters als auch im Rahmen von weiteren Untersuchungen (vgl. Kalb-Müller 2019) wurde festgestellt, dass die Vermittlung über persönliche Kontakte der formalen Vermittlung über entsprechende Institutionen wie der Agentur für Arbeit eher unvermittelt gegenübersteht. „Traditionelle Kontaktpartner und Kanäle konnten nicht zur Vermittlung geeigneter Kandidaten angesprochen werden“ (Hofmann/Dunschen 2017: 203). Kalb-Müller beschreibt wie im ländlichen Raum Kontakte zwischen Geflüchteten und kleineren und mittleren Betrieben über das Engagement Ehrenamtlicher entstanden und im sozialen Netzwerk der dörflichen oder kleinstädtischen Gemeinde vermittelt wurden. Auf der anderen Seite ist es nach Aussage des Unternehmensvertreters äußerst schwierig, sich über mögliche Bewerber*innen und die Möglichkeiten der Anstellung zu informieren. Insbesondere juristische Fragen seien im Zuge dessen zu klären. Dieses ist auch im nächsten Schritt, der Begleitung der Ausbildung oder der Erwerbstätigkeit ein zentrales Thema. So führen Angaben zu Familienstand und Kindern in Arbeitsverträgen immer wieder zu Schwierigkeiten z. B. im Hinblick auf die korrekte Abführung von Steuern. Hinzu kommen individuelle Themen bei Veränderungen des Aufenthaltsstatus oder der Wohnungssuche, die ebenfalls Einfluss auf das Ausbildungs- oder Arbeitsverhältnis hätten. Der Unternehmensvertreter sieht das Unternehmen als Arbeitgeber diesbezüglich als zu wenig von staatlicher Seite unterstützt. - Die gesetzliche Verpflichtung 
der Betreuung der Menschen endet in vielen Fällen nach einer ersten Vermittlung in ein Ausbildungs- bzw. Arbeitsverhältnis. Sozialwirtschaftliche Unternehmen haben lediglich im Rahmen der sozialraumorientierten Arbeit die Möglichkeit, eine weitere Unterstützung der Geflüchteten in weiterhin schwierigen Lebenslagen zu leisten.

Von Seiten des Profit-Unternehmens wurden darüber hinaus folgende Einflussfaktoren in den beschriebenen Prozessen genannt:

- es gibt lokal unterschiedliche Zuständigkeiten bei den jeweiligen kommunalen Behörden;

- in den einzelnen Niederlassungen des international verzweigten Unternehmens bestehen unterschiedliche Unternehmenskulturen im Hinblick auf den Umgang mit und das Ausfüllen von Sozialen Kooperationen (mal steht eher die Kosten/Nutzen-Betrachtung, mal eher die gesellschaftliche Verantwortung im Mittelpunkt);

- es stellt sich zudem immer wieder die Frage, ob über den eigenen Bedarf hinaus ausgebildet werden sollte oder Praktika angeboten werden sollen und

- wie mit unterschiedlichen Sprachniveaus und Ausbildungsständen umgegangen werden kann und muss.

Neben Fragen der Integration in das Unternehmen ist es demnach vor allem die Zusammenarbeit mit den kommunalen Behörden, die das Profit-Unternehmen vor Herausforderungen stellt. Dies um so mehr, da auch die Niederlassungen des Unternehmens diesbezüglich von unterschiedlichen Unternehmenskulturen geprägt sind. An einem Unternehmensstandort gemachte Erfahrungen lassen sich nach Aussage des Unternehmensvertreters nicht auf einen anderen übertragen. Akteure der kommunalen Ebene könnten jedoch als Brücken zwischen privatwirtschaftlichen Unternehmen und Betroffenen agieren (vgl. BBSR 2015: 6). Gestützt auf eine Untersuchung zum Engagement von Unternehmen und Stiftungen für eine soziale Quartiersentwicklung wurde festgestellt:

„Für diese Aufgabe waren auf Seiten der Kommune Mitarbeiterinnen oder Mitarbeiter notwendig, die sich nicht nur in den Quartieren und im Kontakt mit den dortigen sozialen Projekten gut auskennen, sondern die auch einen partnerschaftlichen Kontakt mit Unternehmen und Stiftungen aufbauen können und sich insofern auch im zivilgesellschaftlichen und unternehmerischen Sektor zurechtfinden“" (BBSR 2015: 6).

Für diese an dieser Stelle beschriebene Vermittlerrolle und die hierfür notwendigen Kompetenzen wird in der Profession der Sozialen Arbeit im Schwerpunkt der Sozialraumorientierung das Quartiersmanagement ausgebildet (vgl. Herrmann 2019: 109ff). Unabhängig davon, dass im Rahmen von Kooperationen in den jeweiligen Systemen Funktionsträger mit den entsprechenden Kompetenzen vorhanden sein müssen, ist die Sozialwirtschaft mit den genannten Kompetenzen in diesem Beispiel insbesondere der Arbeitsvermittlung und 
der Orientierungshilfe in den Systemen (Stichwort: ,lost in systems“") prädestiniert dafür, eine Vermittlerrolle zwischen den Akteuren der Kommune und Profit-Unternehmen einzunehmen.

\section{Zum Schluss}

Während veränderte Auftragsbeziehungen zwischen der öffentlichen Hand und sozialwirtschaftlichen Unternehmen wie auch der Profitwirtschaft im Rahmen des Neuen Steuerungsmodells oder auch der Entwicklung einer sozialräumlichen Sozialen Arbeit bereits seit den 1980er Jahren erprobt werden, sind Kooperationen zwischen mehr als zwei Partnern, d. h. zwischen öffentlicher Hand, profit- ebenso wie sozialwirtschaftlichen Unternehmen und zivilgesellschaftlichen Akteuren bisher wenig erprobt. Kooperationen (zwischen unterschiedlichen Sektoren) finden vor allem zwischen sozialwirtschaftlichen und profitwirtschaftlichen Unternehmen als Soziale Kooperationen statt und dieses auch in wachsendem Maße (vgl. Tab. 1). Das Beispiel eines um die Integration von Geflüchteten in die Ausbildung und den Arbeitsmarkt bemühten profitwirtschaftlichen Unternehmens hat jedoch gezeigt, dass ein Drei- bzw. Vierklang zwischen staatlichen, profit- und sozialwirtschaftlichen sowie zivilgesellschaftlichen Akteuren notwendig wäre, nicht zuletzt um innovative Wege (z. B. der Ausbildungs- und Arbeitsvermittlung) in der Bewältigung von aktuellen gesellschaftlichen Herausforderungen zu entwickeln. Bestehende Problematiken und Hindernisse sind dabei oft nur aus den unterschiedlichen Perspektiven der „Systeme“ (politisch-administratives System, Wirtschaft, Sozialwirtschaft, Zivilgesellschaft und nicht zuletzt der Betroffenen selbst) und im konkreten lokalen Kontext zu identifizieren. Akteure der Sozialwirtschaft bzw. der Sozialen Arbeit, ebenso wie die Wissenschaft ${ }^{9}$ könnten in dem Prozess der Identifizierung und Bewältigung von Problemen eine Vermittlungsrolle einnehmen.

\section{Literatur}

Arnold, U./Maelicke, B. (2014): Vorwort zur 1. Auflage. In: Arnold, U./Grunwald, K./Maelicke, B. (Hrsg.): Lehrbuch der Sozialwirtschaft. 4. Aufl. Baden Baden: Nomos. S.11-13.

BBSR (Bundesinstitut für Bau-, Stadt- und Raumforschung) (Hrsg.): Unternehmen und Stiftungen für die soziale Quartiersentwicklung. BBSR-online-Publikationen,

9 So geschehen im Rahmen der Tätigkeit der Autorin am FGW (Forschungsinstitut für gesellschaftliche Weiterentwicklung) im Bereich der Integrierenden Stadtentwicklung (ISE). 
2015, 13. https://www.bbsr.bund.de/BBSR/DE/Veroeffentlichungen/BBSROnline/2015/DL_ON132015.pdf?_blob=publicationFile\&v=2 [Zugriff 22.07.19].

BMAS (Bundesministerium für Arbeit und Soziales) (2010) (Hrsg.): Empfehlungsbericht des Nationalen CSR-Forums an die Bundesregierung. http://www.bmas.de/SharedDocs/Downloads/DE/PDF-Publikationen/a397-csrempfehlungsbericht.pdf?_blob=publicationFile [Zugriff: 18.07.2019].

Braun, S. (2010): Monitor Engagement. Bürgerschaftliches Engagement von Unternehmen in Deutschland. Zwischen Tradition und Innovation. Bundesministerium für Familie, Senioren, Frauen und Jugend (Hrsg.) https://www.bmfsfj.de/blob/94366/a825c94ab072530d175e24b3b210bc56/monitor-engagement-nr-3-data.pdf [Zugriff: 18.07.2019].

Braun, S. (2008): Gesellschaftliches Engagement von Unternehmen in Deutschland. In: APuZ (Aus Politik und Zeitgeschehen) 2008, 31. Bundeszentrale für politische Bildung (Hrsg.). Frankfurt. S. 6-14.

Damm, D./ Lang, R. (2002): Handbuch Unternehmenskooperation - Erfahrungen mit Corporate Citizenship in Deutschland. In: Stiftung Mitarbeit (Hrsg.): UPL-Bundesinitiative. Nr. 7. Bonn, Hamburg.

Empirica ag (2017): Integration von Zuwanderern - Herausforderungen für die Stadtentwicklung. Forschungsprojekt durchgeführt im Auftrag des BBSR (Bundesinstitut für Bau-, Stadt- und Raumforschung) www.bbsr.bund.de [Zugriff 28.05.18].

Evers, A. (2002): Zusammenfassung und Auswertung der Befunde. In: Evers, A./Rauch, U./Stitz, U. (Hrsg.): Von öffentlichen Einrichtungen zu sozialen Unternehmen. Hybride Organisationsformen im Bereich sozialer Dienstleistungen. Berlin: Edition sigma, S. 217-265.

Grunwald, K. (2014): Sozialwirtschaft. In: Arnold, U./Grunwald, K./Maelicke, B. (Hrsg.): Lehrbuch der Sozialwirtschaft. 4. Aufl. Baden Baden: Nomos. S. 33-63.

Habisch, A./Schwarz, C. (2015): CSR als Investition in Human- und Sozialkapital. In: Schneider, A./Schmidpeter, R. (Hrsg.): Corporate Social Responsibility. Verantwortungsvolle Unternehmensführung in Theorie und Praxis. Berlin/ Heidelberg: Springer. S. 113-133.

Herrmann, H. (2001): Initiierte Bürgerforen - Bürgerbeteiligung im Rahmen Sozialer Stadtentwicklung in Hamburg. In: Haus, M. (Hrsg.): Bürgergesellschaft, soziales Kapital und lokale Politik, Reihe Stadtforschung aktuell, Band. X, Opladen: Leske + Budrich.

Herrmann, H. (2019): Soziale Arbeit im Sozialraum. Stadtsoziologische Zugänge. Reihe „Grundwissen der Sozialen Arbeit“. Stuttgart: Kohlhammer Verlag.

Hofmann, H./Dunschen, S. (2017): Globale Megatrends und lokale unternehmerische Verantwortung: Integration von Geflüchteten. In: Bungard, P./Schmidpeter, R. (Hrsg.): CSR in Nordrhein-Westfalen. Nachhaltigkeits-Transformation in der Wirtschaft, Zivilgesellschaft und Politik. Berlin: Springer, Gabler. S. 193-206.

Hollmann, D./Schlicher, C. (2015): Gesellschaftliche Verantwortung der Unternehmen. Ergebnisse der Umfrage. Herausgegeben durch die Bertelsmann Stiftung. Gütersloh.

Kalb-Müller, L. (2019): Herausforderungen ehrenamtlicher Flüchtlingshilfe im ländlichen Raum. In: Alisch, M. (Hrsg.): Zwischenräume - Sozialraumentwicklung in der Migrationsgesellschaft. Opladen, Berlin, Toronto: Barbara Budrich. S. 115-134. 
Kleine-König, Christiane (2016): Unternehmen engagieren sich in der Nachbarschaft. Verantwortung findet Stadt. In: CSR MAGAZIN. Unternehmen, Verantwortung, Gesellschaft, 2016, 1, S.28-30.

Kleine-König, C. (2018): Stadtentwicklung als Handlungsfeld für gesellschaftlich engagierte Unternehmen. In: Backhaus-Maul, H./Kunze, M./Nährlich, S. (Hrsg.): Gesellschaftliche Verantwortung von Unternehmen in Deutschland. Wiesbaden: Springer VS. S. 301-316.

Lang, R./Dresewski, F. (2010): Zur Entwicklung des Social Case zwischen Unternehmen und Nonprofit-Organisationen. In: Backhaus-Maul, H./ Biedermann, C./Nährlich, S./Polterauer, J. (Hrsg.): Corporate Citizenship in Deutschland. Gesellschaftliches Engagement von Unternehmen. Bilanz und Perspektiven. 2. Aufl. Wiesbaden: VS Verlag für Sozialwissenschaften, S. 401-422.

Lang, R./Kromminga, P./Dresewski, F. (2007): Wie Unternehmenskooperation die Problemlösungskompetenz Sozialer Arbeit stärkt. UPJ-Arbeitspapier, Berlin. www.soziale-kooperation.de [Zugriff: 11.01.2016].

Oschmiansky, F. (2010). Neues Steuerungsmodell und Verwaltungsmodernisierung. http://www.bpb.de/politik/innenpolitik/arbeitsmarktpolitik/55048/steuerung-modernisierung [Zugriff: 19.08.2019].

Rasche, A./Morsing, M./Moon, J. (Hrsg.) (2017): Corporate Social Responsibility. Strategy, Communication, Governance. Cambridge, New York: Cambridge Uni. Press.

Richtlinie 2014|95|EU des Europäischen Parlaments und des Rates vom 22. Oktober 2014 zur Änderung der Richtlinie 2013|34|EU im Hinblick auf die Angabe nichtfinanzieller und die Diversität betreffender Informationen durch bestimmte Unternehmen und Gruppe (ABl. vom 15.11.2014, L330/1-L330/9).

Riess, B. (2010): Unternehmensengagement - ein Beitrag zur gesellschaftlichen Selbststeuerung zwischen Markt und Staat. In: Backhaus-Maul, H./Biedermann, Ch./Nährlich, St. (Hrsg.): Corporate Citizenship in Deutschland. Gesellschaftliches Engagement von Unternehmen. Bilanz und Perspektiven. 2. aktualisierte Aufl. Wiesbaden: VS Verlag. S. 588-600.

Stoll, B./Herrmann, H. (2018): Soziale Teilhabe - Unternehmerische Verantwortung. Corporate Social Responsibility/ Corporate Citizenship - Potenziale zur Stärkung der sozialen Teilhabe von Menschen \& Gruppen. https://www.hs-fulda.de/sozialwesen/forschung/cesst/forschen/forschungsprojekte/ [Zugriff: 19.12.2018].

Stoll, B. (2009): Sozial und ökonomisch handeln. Corporate Social Responsibility kleiner und mittlerer Unternehmen. Frankfurt/New York: Campus Verlag.

Weingarten, J./Wohlert, J. (2018): Integration Geflüchteter in Arbeitsmärkte. Potentiale und Herausforderungen. In: Standort. Zeitschrift für angewandte Geographie 2018, 42, S. 51-55. Berlin, Heidelberg: Springer. https://doi.org/10.1007/s00548-0180519-z [Zugriff:19.05.2019]. 


\section{Gemeinsam mehr bewegen - Erfolgsfaktoren für Kooperationen zwischen sozialen Organisationen und Wirtschaftsunternehmen in Zeiten von CSR}

Karl-Hans Kern

\section{Vorbemerkung}

Wer sich seitens der Caritas der Aufgabe widmet Solidarität in der Gesellschaft zu fördern und dabei Unternehmen als Partner*innen gewinnen will, um gemeinsam Aufgaben zu bearbeiten die jeder für sich alleine genommen nicht umsetzen könnte, der kann auf Erfahrungen aus der 14 Jahre bestehenden CSR-Kooperation zwischen der Hyundai Motor Deutschland GmbH und der Caritas zurückgreifen. Denn unabhängig davon, ob es sich bei den potenziellen Partner*innen um kleine oder mittlere Unternehmen oder um Großkonzerne handelt, das grundsätzliche Vorgehen und die Erfolgsfaktoren für ein Gelingen von Kooperationen sind die Gleichen. Es werden Ziele geklärt, strategische Entscheidungen getroffen, Vertrauen aufgebaut, Beziehungen entwickelt und gestaltet. Dafür müssen die Beteiligten finanzielle und personelle Ressourcen bereitstellen, die für alle Partner*innen passenden Angebote entwickeln und Verständnis für die Ziele des jeweils anderen aufbringen. Mit den Wirkungen, die beide Partner*innen für die Gesellschaft erzielen, wird aus der win-win Situation ein Gewinn für alle drei Beteiligten, für die soziale Organisation, für das Unternehmen und für die Gesellschaft.

\section{Mit Unternehmen kooperieren - die Erfolgsfaktoren}

Strategische und langfristige Partnerschaften zwischen der Caritas und Unternehmen der Privatwirtschaft, also das geplante und in den Organisations- und Unternehmensstrukturen verankerte bewusste Miteinander, sind bundesweit noch im Aufbau begriffen. Seit Jahren etabliert hat sich hingegen an vielen Stellen schon die Durchführung von Corporate Volunteeringmaßnahmen, bei 
denen sich Mitarbeiterinnen und Mitarbeiter aus Unternehmen für soziale Belange in Einrichtungen und Diensten der verbandlichen Caritas engagieren. Auch die Unterstützung einzelner Aktionen der Caritas mit Geld- und Sachleistungen von Unternehmen, ist Bestandteil der Alltagsarbeit in den Verbänden und Organisationen. Auf lokaler Ebene werden somit tagtäglich Kooperationen umgesetzt und daran gearbeitet, aus einem oftmals noch einmaligen Miteinander, langfristige Partnerschaften zu entwickeln. Dabei rücken zwei Fragen immer wieder in den Mittelpunkt:

Wie kann die Zusammenarbeit dieser beiden Welten mit ihren unterschiedlichen Zielsetzungen und Rahmenbedingungen gelingen?

Welches sind Erfolgsfaktoren, die dazu beitragen, die ,Win-Win-Win Situation" herbeizuführen und die gemeinsame Bearbeitung sozialer Aufgaben erfolgreich zu ermöglichen?

Die Beantwortung der Fragen verlangt zunächst deren Einordnung in die Entwicklung von Corporate Social Responsibility (CSR), der gesellschaftlichen Verantwortung von Unternehmen in Deutschland und die damit verbundenen Herausforderungen für Unternehmen und soziale Organisationen.

\section{Corporate Social Responsibility (CSR) - Verantwortung von Unternehmen für die Gesellschaft}

Auf der Grundlage der Leitsätze des „ehrbaren Kaufmanns“ übernahmen Unternehmen, allen voran die familiengeführten, schon immer Verantwortung für ihr Wirken in der Gesellschaft. Sie schafften Arbeitsplätze und damit die Existenzgrundlage für Arbeitnehmende und ihre Familien. In vielen Fällen stellten sie darüber hinaus auch Ressourcen ihres Unternehmens zur Verfügung, um z.B. sportliche, soziale und kulturelle Vorhaben an ihrem Unternehmensstandort zu fördern. Doch mit der Weiterentwicklung von CSR einher geht die Tatsache, dass Geschäftsführungen, Inhaber*innen und Verantwortliche in Unternehmen deren gesellschaftliches Engagement stärker als bisher strategisch ausrichten.

Es geht ihnen nicht mehr nur um gute Öffentlichkeitsarbeit und Imagewerbung sondern auch darum, für Mitarbeiterinnen und Mitarbeiter ein attraktiver Arbeitgebende zu sein, sich gegenüber Lieferant*innen und Kunden*innen als vertrauenswürdig und zuverlässig zu beweisen und Maßnahmen einzuleiten, um die Umweltbelastungen zu reduzieren und dabei auch noch die Ausgaben des Unternehmens zu senken. Damit wird das gesellschaftliche Engagement $\mathrm{zu}$ einer Unternehmensstrategie, mit dem Ziel einer nachhaltigen und wirtschaftlich erfolgreichen Absicherung und Weiterentwicklung des Unternehmens (vgl. CSR-Kompetenzzentrum im Deutschen Caritasverband 2016: 4) 
CSR ist also keineswegs das „Sahnehäubchen“ in einem gut funktionierenden Unternehmen. Denn CSR befasst sich nicht mit der Frage, wofür Unternehmen ihren Gewinn einsetzen. Vielmehr steht die Frage im Mittelpunkt, wie Unternehmen ihre Gewinne in ihrem Kerngeschäft erwirtschaften. Es handelt sich also bei CSR um eine zeitgemäße und nachhaltige Unternehmensführung. Mehr denn je müssen Unternehmen dabei aber den Beweis antreten, dass sie die von ihnen publizierten Werte nicht nur schätzen, sondern sie in ihrer Geschäftspraxis tatsächlich auch einhalten und konkrete Maßnahmen einführen, um dies zu gewährleisten. Unternehmen stehen unter ständiger Beobachtung immer interessierter und kritischer werdender Anspruchsgruppen, die aus möglichen Fehlern umgehend Konsequenzen ziehen. Dies gilt übrigens zunehmend auch für soziale Organisationen und die Umsetzung ihrer Aufgaben.

\section{Die Rolle als CSR-Partner}

Wenn sich Unternehmen an ihren Standorten zunehmend mit Mitarbeiterinnen und Mitarbeitern oder in anderer Form in das Gemeinwesen einbringen wollen, benötigen sie für ihre CSR-Maßnahmen geeignete Partner*innen. Organisationen die wissen, welche sozialen Herausforderungen anstehen und die das Know-how und die Bereitschaft zu deren gemeinsamer Bearbeitung mitbringen.

„Sich gemeinsam mit Unternehmen gesellschaftlichen Herausforderungen anzunehmen und sich aus unterschiedlichen Blickwinkeln und mit unterschiedlichen Kompetenzen und Ressourcen deren Bearbeitung anzunähern, ist Ausdruck des Leitgedankens der Caritas zur Förderung der Solidarität in der Gesellschaft. Für die Förderung und Vermittlung von Unternehmenskooperationen haben wir deshalb im Jahr 2011 gemeinsam mit verbandlichen Partnern das CSR-Kompetenzzentrum im Deutschen Caritasverband ins Leben gerufen." (Millies 2018: 8).

Der Caritasverband der Diözese Rottenburg-Stuttgart hatte schon einige Jahre vor der Gründung des CSR-Kompetenzzentrums seine Zusammenarbeit mit Unternehmen intensiviert und 2004 mit dem damals noch in Neckarsulm angesiedelten und zur Schweizer Emil Frey Gruppe gehörenden Fahrzeugimporteur, der Hyundai Motor Deutschland $\mathrm{GmbH}$, eine Kooperation ins Leben gerufen. Darüber hinaus war er von 2011 bis 2018 Träger des o.g. CSR-Kompetenzzentrums im Deutschen Caritasverband und hielt mit ihm personelle und finanzielle Ressourcen bereit, um für Unternehmen bundesweit Ansprechpartner zu sein und Kooperationen, wie die mit Hyundai Motor Deutschland, begleiten und koordinieren zu können.

Kooperieren heißt, sich auf andere einzulassen, die Ziele des Partners mitzutragen und mit zu verantworten. Kooperieren heißt auch, voneinander zu ler- 
nen, die Dinge aufgrund des Miteinanders anders zu machen, nämlich in Abstimmung und unter Berücksichtigung der Erwartungen und Vorstellungen des jeweils Anderen. Das sind die Eckpunkte dieser langjährigen Zusammenarbeit. Sie wurden sowohl von Hyundai als auch der Caritas im Laufe der Zeit geschärft und an aktuelle Entwicklungen im Miteinander angepasst. Dabei stand der Begriff der Partnerschaft im Vordergrund. Ein Miteinander, das gemeinsam gestaltet und nur gemeinsam erfolgreich umgesetzt werden konnte.

\section{Hyundai Motor Deutschland GmbH}

Die Hyundai Motor Deutschland GmbH ist inzwischen eine 100 prozentige Tochter der Hyundai Motor Company und sein Unternehmensstandort befindet sich in Offenbach. In Deutschland hat Hyundai im Jahr 2017 mit einem Marktanteil von 3,2 Prozent, 108.518 Zulassungen und einem Privatkundenanteil von 50 Prozent eine hohe Qualität der Zulassungen erreicht. Damit konnte sich Hyundai in den Top Drei der Importmarken etablieren und bleibt stärkste asiatische Marke in Deutschland. Das Unternehmen wurde vom Automotive Brand Contest für seine nachhaltige und konsequente Entwicklung in Deutschland als Marke des Jahres ausgezeichnet. Das Unternehmen versteht sich nicht nur als Wirtschaftsunternehmen, sondern als aktives Mitglied der Gesellschaft, das Verantwortung trägt und übernimmt. Daher ist Hyundai als langjähriger Partner der FIFA sowie der Caritas im sportlichen und im sozialen Bereich engagiert. ${ }^{1}$

\section{Gemeinsam mehr bewegen - Die Projekte}

Die Zusammenarbeit der beiden Partner begann im Vorfeld der Fußballweltmeisterschaft in Deutschland. „Die Welt zu Gast bei Freunden“ lautete der damalige Slogan der FIFA, der das Fußballereignis begleiten sollte. Das vorherige Jahrzehnt war in Deutschland allerdings mit zahlreichen fremdenfeindlichen Übergriffen zu Ende gegangen. Flüchtlinge und Menschen aus anderen Kulturkreisen wurden auf offener Straße erschlagen und deren Unterkünfte angezündet. Daher trat die Caritas an, mit einem bundesweiten Straßenfußballturnier für Fairplay und Miteinander zu werben und so ihrerseits einen Beitrag dazu zu leisten, die Aussage der FIFA mit Leben zu füllen.

1 Die Kooperation wurde Ende 2018 nicht mehr verlängert. 
Eine Einladung zur Durchführung eines bundesweiten Straßenfußballturniers richtete die Caritas auch an die Hyundai Motor Deutschland GmbH. Von dort erhielt sie nicht das Standardschreiben, mit dem rund fünfzig andere angefragte, international tätige Unternehmen die Einladung abgelehnt hatten. Es kam zur Kontaktaufnahme und nach ausführlichen Verhandlungen unterzeichneten die Partner 2004 die Vereinbarung einer Zusammenarbeit.

Seither warben die Partner unter dem Titel „Gemeinsam mehr bewegen“ mit einem bundesweiten Fußballturnier für Toleranz und Weltoffenheit, förderten die Bildung junger Menschen und stärkten sie in ihrer persönlichen Entwicklung. Sie unterstützten ehrenamtlich Tätige bei der Umsetzung ihrer Vorhaben mit einen „Ehrenamtsfonds“, stellten Einrichtungen der Jugendhilfe Kleinbusse zur Verfügung und organisierten Fahrsicherheits- und Spritspartrainings für Sozialstationen und deren Mitarbeitenden.

Nach einer gemeinsamen Reflexion der bisherigen Zusammenarbeit und des damit verbundenen Erreichten, wurde die bestehende Konzeption zum Vertragszeitraum 2017/2018 fortgeschrieben. Zukünftig sollten die Unterstützungsleistungen des Unternehmenspartners gebündelt dort eingesetzt werden, wo es in der Vergangenheit schon Verbindungen zu Hyundai gab oder ein explizites Interesse an einer Partnerschaft mit Hyundai zum Ausdruck gebracht wurde. Damit wollte man den Partnerschaftsgedanken fördern und die Zusammenarbeit festigen.

Mit dieser konzeptionellen Veränderung einher ging eine Ausweitung der konkreten Hilfen für in Not geratene Menschen und eine Verlagerung des Schwerpunktes der Hilfen auf das Bundesland Hessen. Gleichzeitig wurde die Berichterstattung über die realisierten Projekte intensiviert. Diese Maßnahmen hatten einerseits zum Ziel „Gutes zu tun und darüber zu reden“, und so z.B. auch andere Unternehmen $\mathrm{zu}$ motivieren, sich zu engagieren. Andererseits diente die Kommunikation bei beiden Partnern intern dazu, die Akzeptanz für das Miteinander zu stärken und das bundesweite Interesse am Miteinander von Caritas und Hyundai zu fördern.

\subsection{Hyundai/Caritas Spielmobil}

Gerade in Ballungsräumen, wie in Offenbach, ist es für Kinder nicht mehr selbstverständlich, sich mit Freunden draußen zum Spielen und Toben zu treffen. In manchen Stadtteilen fehlt es an Spielplätzen und -gelegenheiten. Deshalb konzipierte Hyundai gemeinsam mit der Caritas ein Spielmobil, das durch den Caritasverband Offenbach/Main e.V. eingesetzt wurde. Es diente als regelmäßige und sichere Anlaufstelle zum unbeschwerten Spielen und bot Kindern den Zugang zu Spielgeräten. Mit dem Spielmobil erreichte die Caritas die Familien direkt in ihren Stadtteilen. Während die Kinder zusammen spielten, 
kam die Caritas mit Eltern in Kontakt und konnte ihnen bei Bedarf Hilfe- und Unterstützungsangebote aufzeigen.

\subsection{Das Hospiz-Leben ist angesagt}

Von „Gemeinsam mehr bewegen“ unterstützt wurden auch drei Hospize in Taunusstein, Oberursel und Gelnhausen. Ein Kinobesuch, eine Einladung zum Abendessen bei Freunden, ein Ausflug ins Museum - die Gäste des Hospizes St. Elisabeth Kinzigtal hatte in der letzten Phase ihres Lebens ganz alltägliche Wünsche. Dass diese erfüllt werden konnten, dafür sorgte Hyundai mit der Übergabe eines Hyundai IONIQ Hybrid. Mit ihm wurde die Mobilität für Ausflüge, aber auch tägliche Besorgungen gesichert. Damit blieb den Gästen des Hospizes ein Stück Normalität und Lebensqualität auch in der letzten Phase ihres Lebens erhalten.

Sterben ist intensives Leben. Dieser Satz aus dem Mund einer Pflegefachkraft im Caritas Hospiz in Oberursel brachte den Geist der Einrichtung zum Ausdruck. Es gehe darum, den Gästen des Hospizes einen würdigen Abschied vom Leben zu ermöglichen. Ein hochmoderner Pflegesessel biete mobilitätseingeschränkten Menschen die Möglichkeit, doch noch mit ihren Angehörigen im Haus, der Terrasse oder im Garten unterwegs sein zu können.

Die Sehnsucht, Boden unter den Füßen zu spüren, begleitet viele Gäste im Hospiz St. Ferrutius in Taunusstein. Zwei Niederflurbetten ermöglichen es ihnen nun zukünftig, ohne Sturzgefahr den Kontakt zur Erde herzustellen.

\subsection{Spende ein Licht}

Unter dem Motto „Spende ein Licht“ hatten Hyundai und Caritas in der Adventszeit dazu aufgerufen, Kerzen zu basteln, um sie so im wahrsten Sinne des Wortes zum Strahlen zu bringen. 655 Kinder und ihre Familien beteiligten sich und schickten ihre Kerze ein. Die Spende von $10 €$ pro Kerze rundete Hyundai auf $12.000 €$ auf. Mit der Spende wurde die Arbeit der Caritas in Offenbach mit Kindern suchtkranker Eltern sowie die Anschaffung von Bücherkisten für Lesepaten von Kinderstiftungen in Oberschwaben unterstützt. Damit fördern ehrenamtlich Tätige unter dem Dach der Caritas-Initiative „Mach dich stark“ die Lesebereitschaft und -fähigkeit von Kindern aus benachteiligten Familien. 


\section{Erfolgsfaktor Haltung: „Partner statt Bittsteller“}

In der im August 2018 veröffentlichten Arbeitshilfe des Caritas CSR-Kompetenzzentrums mit dem Titel: ,Gemeinsam mehr bewegen - Das Einmaleins der Unternehmenskooperationen am Beispiel der bundesweiten CSR-Kooperation der Caritas mit der Hyundai Motor Deutschland GmbH“ wird das Thema „Partner statt Bittsteller“ aufgegriffen und wie folgt beschrieben:

„Wer sich aus der Haltung heraus „Wir tun Gutes und dazu brauchen wir Geld - Unternehmen haben Geld und wollen Gutes tun" darum bemüht, finanzielle Mittel von Unternehmen einzuwerben, ist auf UnternehmerInnen oder Unternehmen angewiesen, die eher aus altruistischer Motivationslage heraus handeln oder weil die bisherige Spendenpraxis des Unternehmens diese Form der Unterstützung der sozialen Organisation ermöglicht. In der Konsequenz allerdings werden damit Unternehmen auf eine Funktion als Geldgeber reduziert. Ein Bild, dem Unternehmen mit wachsender strategischer Ausrichtung ihrer Corporate Social Responsibility immer weniger entsprechen (wollen) [und das in ihrer Konsequenz die Rolle der Caritas als „Bittsteller“ um Unternehmensressourcen festigt]. Wer aber Unternehmen als Partner gewinnen will und längerfristige Partnerschaften mit ihnen anstrebt, wird erst einmal klären, was der Begriff Partner für ihn bedeutet. Was einen Partner ausmacht, lässt sich sehr gut aus Erkenntnissen zu Partnerschaften zwischen Menschen ableiten. Überhaupt sind Kooperationen der Caritas mit Unternehmen an vielen Stellen mit dem Kennenlernen und dem sich aufeinander Einlassen von Menschen vergleichbar. Daher greifen wir auch gerne auf eine Quelle zurück, die beschreibt, was ältere Paare antworteten, wenn man sie nach den Erfolgsrezepten einer glücklichen Partnerschaft fragt:

„Das Erfolgsrezept für eine glückliche lange Beziehung ist schwer zusammenzustellen - zu unterschiedlich sind die Menschen und ihre Lebensumstände. Fragt man ältere Paare nach den Zutaten, antworten sie oft mit: „Respekt“, „Achtung“, „Wertschätzung“, „,den anderen so akzeptieren, wie er ist". Anders als Liebe ist eine Partnerschaft planbar. Sie lässt sich gestalten und wir können - und müssen auch - an ihr arbeiten. Für eine gute Partnerschaft muss allerdings schon ein Minimum an Gemeinsamkeiten vorhanden sein. Ein bestimmtes gemeinsames Grundverständnis, ähnliche Wertvorstellungen und Weltanschauungen, gemeinsame Interessen und Erlebnisse... Wer viel gemeinsam hat, kommt besser über die Runden". (Andrea Wengel, Liebe und Partnerschaft folgen eigenen Gesetzen, www.planet-wissen.de, ARD, SWR alpha.)“ (CSR-Kompetenzzentrum im Deutschen Caritasverband 2018:3)

Dieses Grundverständnis spiegelte sich in der Zusammenarbeit der Caritas und Hyundai wider. Welche Maßnahmen geplant und welche Projekte damit unterstützt werden, wurde in gemeinsamen Besprechungen der beteiligten Verantwortlichen geklärt. Der Bedarf der Caritas einerseits und das Interesse Hyundais an der Generierung positiver Kommunikationsanlässe wurden so miteinander verknüpft.

Im Laufe der Zusammenarbeit wuchs das Wissen und das Verständnis für die Logiken und Handlungsweisen des jeweils anderen und es entwickelte sich das für das Miteinander notwendige Vertrauensverhältnis. Vertrauen machte es möglich, auftretende Konflikte, die sich aus unterschiedlichen Interessenslagen durchaus ergeben können, offen anzusprechen und unter Einbeziehung 
der Beteiligten einer Lösung zuzuführen. Vertrauen ist damit ein wesentlicher Bestandteil für ein gelingendes Miteinander in jeder Kooperation. Und Partnerschaft ist dabei der Ausdruck eines Miteinanders auf Augenhöhe.

\section{Erfolgsfaktor Handwerk: „Feste Ansprechpartner“6}

Der aktive Auf- und Ausbau von Unternehmenspartnerschaften kann nicht „nebenher“ erfolgen. Das bestätigten 2016 auch im Rahmen einer bundesweiten Befragung für Unternehmenskooperationen die verantwortlichen Mitarbeitenden der Caritas. Die Mitwirkung in Unternehmensnetzwerken, die Herstellung von Unternehmenskontakten und deren Festigung, die Umsetzung von Maßnahmen und die Pflege der Beziehung zu den Unternehmen verlangt die Bereitstellung entsprechender personeller Ressourcen und die Bereitschaft Geduld und Zeit einzubringen. Bundesweite Erfahrungen aus Unternehmenskooperationen zeigen übrigens, dass dies sowohl für soziale Organisationen als auch gewerbliche Unternehmen gilt, die Kooperationen mit sozialen Organisationen anstreben.

Für die Projektumsetzung gab es sowohl auf der Seite Hyundais als auch der Caritas feste Ansprechpartner*innen. Die Bedeutung einer von Vertrauen, Verbindlichkeit und Sympathie gekennzeichneten Beziehung der Verantwortlichen auf der Arbeitsebene zueinander, darf bei der Realisierung von Unternehmenskooperationen nicht unterschätzt werden. Sich zu kennen, Vertrauen und Verbindlichkeit zu erfahren und konstruktive Kritik äußern und damit umgehen zu können, ist einer der zentralen Erfolgsfaktoren.

\section{Nichts ist statisch}

Partnerschaften verlaufen nicht nach starren Mustern. Auch nicht die Kooperation von Caritas und Hyundai Motor Deutschland. Die geschilderten Erfahrungen und die Erkenntnisse aus der bundesweiten Zusammenarbeit der Caritas mit Unternehmen bilden quasi ein Puzzle, das zusammengefügt ein Bild ergibt. Nicht immer beginnt man mit der Erstellung des Puzzles am Rande, weil sich vielleicht gerade im Mittelpunkt des Bildes Puzzleteile zusammenfügen. Wichtig ist, sich die Flexibilität erhalten, und vor allem die Sensibilität, um im richtigen Moment die Chancen zu erkennen. Die eigene Menschenkenntnis zu nutzen und auch auf „Bauchgefühle“ zu vertrauen sind unverzichtbare Elemente im Miteinander sozialer Organisationen und Unternehmen. 
Denn letztendlich stehen hinter allen Entscheidungen Menschen. Und Partnerschaften werden in erster Linie nicht von Organisationen und Unternehmen geprägt, sondern von den Menschen, die sie verantworten.

\section{Die Rolle als CSR Akteur - ein Ausblick}

Wenn CSR nicht die Frage beantwortet, wofür ein Unternehmen sein Geld ausgibt, sondern CSR die Frage stellt, wie ein Unternehmen seinen Gewinn erwirtschaftet, worin bestehen dann Schnittstellen zu einer gemeinnützigen Organisation über deren Partnerrolle hinaus?

CSR hilft Unternehmen ihre Glaubwürdigkeit und Arbeitgeberattraktivität zu erhöhen, unterstützt sie dabei neue Mitarbeiterinnen und Mitarbeiter zu gewinnen und an ihr Unternehmen zu binden und wertet ihre Marke sozial auf. CSR ist für Unternehmen die Antwort auf einen verantwortungsvollen Umgang mit den begrenzten Ressourcen unserer Erde. CSR hilft Unternehmen dabei ihr Kerngeschäft nachhaltig zu stärken.

Ist es in Hinblick auf die Rolle der Caritas als Dienstgeber dann nicht naheliegend zu fragen, ob CSR nicht auch einem kirchlichen Wohlfahrtsverband oder einer sozialen Organisation bei dessen Herausforderungen und Aufgaben dienlich sein könnte oder sie gerade herausfordert?

Anders gefragt, reicht es noch aus unsere Gemeinnützigkeit zu betonen, damit junge Menschen gerne bei uns als haupt- oder ehrenamtliche Tätige arbeiten, Fördernde uns unterstützen oder die Öffentlichkeit in uns und unser Handeln vertraut?

Patrick Hofmacher, Mitglied der Geschäftsführung der Malteser Werke gGmH mit Sitz in Köln, bringt seine Antwort auf all die Fragen mit einem offenen Blick auf die eigene Organisation in einem Satz auf den Punkt „Nur weil wir als katholischer Dienstleister in den Bereichen der Jugendhilfe und Migration Gutes tun, bedeutet das noch nicht, dass wir automatisch auch in anderen Bereichen der Unternehmensführung gut sind." Diese Aussage eines katholischen Arbeitgebenden für rund 700 Mitarbeiterinnen und Mitarbeiter symbolisierte im Herbst 2011 den Beginn eines Prozesses, der mit der Veröffentlichung des ersten Nachhaltigkeitsberichts der Malteser Werke im Juli 2013, nach eigenen Aussagen der „Beginn eines langen Weges“ ist.

Und auch der Diözesancaritasverband Osnabrück hat sich schon 2011 dazu entschieden, eine verbandliche CSR-Strategie zu entwickeln. Als Partnerin des Projektes „Zukunft einkaufen“, befasste sich die Caritas gemeinsam mit dem Diakonischen Werk über die Dauer von zwei Jahren hin mit der Frage des ökofairen Einkaufs. Als Trägerin großer Behinderteneinrichtungen ist ein die Schöpfung bewahrender Einkauf für die Caritas natürlich eine ökologische, aber auch soziale und ökonomische Frage. Zentraler Einkauf, Bevorzugung 
regionaler und lokaler Produkte und Händler*innen, Entwicklung von Alternativen zur Entsorgung von als Sondermüll deklariertem Windelmaterial, bis hin zur Verwendung ökologisch einwandfreier Materialien bei der Sanierung oder dem Neubau von Gebäuden, stellen die Caritas in Osnabrück vor vielfältigste Herausforderungen rund um ihre unternehmerische Verantwortung.

Sich dessen bewusst, weiteten die Verantwortlichen des Verbandes ihren Blick für drei anderen CSR-Themenfelder und befassten sich darüber hinaus u.a. damit, was es heißt auch zukünftig für gesuchte Fachkräfte ein attraktiver Arbeitgebender zu sein, auf Augenhöhe mit Unternehmen zu kooperieren oder als kompetenter und zuverlässiger Dienstleister wahrgenommen zu werden.

Acht Jahre nach der Gründung des CSR-Kompetenzzentrums im Deutschen Caritasverband sind weitere Verbände und Organisationen unter dem Dach der Caritas dabei, ihre Verantwortungs- und Nachhaltigkeitsaktivitäten in die verbandlichen Strategien zu integrieren. Sie verstehen CSR als Chance, ihr Alltagshandeln als kirchlicher Wohlfahrtsverband zu optimieren, es weiter zu entwickeln. Zum Nutzen der Menschen für die die Caritas eintritt, zum Nutzen derjenigen, die sich haupt- und ehrenamtlich in und mit der Caritas engagieren und zum Nutzen der Gesellschaft.

\section{Literatur}

CSR-Kompetenzzentrum im Deutschen Caritasverband (Hrsg.) (2016): Erfolgsgeschichten - Wie Kooperationen von Unternehmen und sozialen Organisationen gelingen. Köln, Stuttgart: JTI.

CSR-Kompetenzzentrum im Deutschen Caritasverband (Hrsg.) (2018): Gemeinsam mehr bewegen. Das Einmaleins der Unternehmenskooperationen am Beispiel der bundesweiten CSR-Kooperation der Caritas mit der Hyundai Motor Deutschland $\mathrm{GmbH}$. Stuttgart.

Millies, H.J. (2018): Finanz- und Personalvorstand des Deutschen Caritasverbandes. In: Gemeinsam mehr bewegen - Das Einmaleins der Unternehmenskooperationen am Beispiel der bundesweiten CSR-Kooperation der Caritas mit der Hyundai Motor Deutschland GmbH, Caritas CSR-Kompetenzzentrum, August 2018, S. 8.

Wengel, A. (o.J.): Liebe und Partnerschaft folgen eigenen Gesetzen. www.planet-wissen.de, ARD, SWR alpha [Zugriff: 17.05. 2017] In: Gemeinsam mehr bewegen Das Einmaleins der Unternehmenskooperationen am Beispiel der bundesweiten CSR-Kooperation der Caritas mit der Hyundai Motor Deutschland GmbH, Caritas CSR-Kompetenzzentrum, August 2018. 


\section{Ansätze zur Verankerung von CSR in profit- und sozialwirtschaftlichen Unternehmen}

Bettina Stoll

\section{Hinführung zur CSR und den Ansätzen (Managementsystemen) zur Verankerung}

Corporate Social Responsibility bzw. unternehmerische Nachhaltigkeit (s.u.) braucht für ihre Verwirklichung in Organisationen gleichermaßen realitätstaugliche wie Visionen tragende und unterbauende Managementsysteme. Unternehmen müssen in diesen Managementsystemen eine Unterstützung finden, Aspekte gesellschaftlich verantwortlichen bzw. nachhaltigen Handelns in den eigenen Strukturen, Prozessen und Ergebnissen zunächst erkennen und dann im positiven Sinne konsequent, aber flexibel, verankern zu können. Entsprechend sollten spezifische Managementsysteme sowohl überschaubarer dynamischer Startpunkt nachhaltiger Überlegungen und Maßnahmen sein (können), als auch möglicher Ausgangspunkt einer umfassenden organisationalen Evolution hin zur nachhaltigen Organisation ${ }^{1}$. Mit den folgenden Ausführungen wird in Augenschein genommen über welche Ansätze bzw. mit Hilfe welcher Managementsysteme Corporate Social Responsibility (CSR), durchaus auch prozesshaft, letztlich als langfristiges Leitkonzept in Unternehmen (die Begriffe „Unternehmen“ und der „Organisation“ werden im Folgenden synonym verwandt) verankert werden kann. Um sich nicht in einer wenig konkretisierenden Aufzählung diverser Instrumente, Tools etc. zu verlieren, werden im Folgenden konkret zwei Ansätze dargestellt, von denen bereits an dieser Stelle grundlegend davon ausgegangen wird, dass sie - wenn auch mit einem unterschiedlichen Zugang - relevante Managementsysteme im Verantwortungs- bzw. Nachhaltigkeitsmanagement sein können (und welche sich auch nicht gegenseitig ausschließen, d.h. beide Ansätze können im Unternehmen Eingang finden (s. Abschn. 4)). Die Darstellungen und Erläuterungen sollen

1 Zu einer möglichen Betrachtungsweise, wann ein Unternehmen eine (un-)nachhaltige Organisation ist (vgl. z.B. Schaltegger 2018: 354). 
damit auch ermöglichen, die beiden Managementansätze in ihrer tatsächlichen Tauglichkeit für die eigene Organisation einschätzen zu können.

Aufgezeigt wird zum einen ein Ansatz, der die mehrperspektivische Betrachtung der Unternehmenstätigkeit nach der Balanced Scorecard (s.u.) in sich birgt. Zum anderen wird mit EMASplus ein zertifizierbarer Ansatz dargestellt, der das international gültige Umweltmanagementsystem EMAS (die DIN EN ISO 14001-2015 einschließend) nutzt, wie auch die ISO 26000 (internationale Norm zur „Gesellschaftlichen Verantwortung von Organisationen) (vgl. UGA o.J. a). Mit der Integration dieser ,Standards“ über EMASplus soll sichergestellt sein, dass die Dreidimensionalität von CSR (s.u.), d.h. die ökonomische, die ökologische und die soziale Perspektive auf das Unternehmen $^{2}$, beachtet wird. Beide Ansätze - BSC und EMASplus - sind als ein konkretes Managementsystem zu verstehen, sind also anders gelagert, als Tools bzw. Sammlungen von solchen, die das CSR-Management, z.B. spezifisch den Einstieg in dieses, erleichtern sollen (und ebenfalls ihren Sinn haben können), wie z.B. das Global Value Toolkit ${ }^{3}$. Ziel der folgenden Ausführungen und Überlegungen ist es, über die Darstellung der Managementsysteme fassbar zu machen, dass und wie es gelingen kann die Leitidee von CSR bzw. Nachhaltigkeit systematisch und langfristig im Unternehmen zu verankern bzw. das Unternehmen mit seiner Geschäftstätigkeit an sich, an CSR/am Nachhaltigkeitsgedanken, auszurichten. Beide Ansätze liefern dabei auch Daten- und Informationsmaterial, welches z.B. in die CSR- bzw. Nachhaltigkeitsberichterstattung eingehen kann.

Ist im Folgenden von Corporate Social Responsibility die Rede, soll damit das speziell auf profit- und sozialwirtschaftliche Unternehmen (zur Unterscheidung s.u.) heruntergebrochene nachhaltige unternehmerische Handeln bezeichnet werden, in der Weise, dass ökonomisches, ökologisches und soziales Handeln die Geschäftstätigkeit des Unternehmens bzw. der Organisation leitet (vgl. Stoll 2009: 59f., DIN 2011: 24). „CSR bezeichnet den spezifischen Beitrag, den Unternehmen zum nachhaltigen Wirtschaften, zur Nachhaltigkeit, leisten" (BMAS o.J.). In diesem Sinne werden im Folgenden auch beide Begrifflichkeiten - CSR und Nachhaltigkeit - verwandt. Corporate Social

Als ein ebenfalls zertifizierbares Managementsystem für gesellschaftliche Verantwortung versteht sich z.B. die Qualitätsnorm DS 49001, ein dänischer ins Deutsche übersetzter Standard (vgl. Dansk Standard 2011).

3 Als Ergebnis eines Forschungsprojektes der Wirtschaftsuniversität Wien steht mit dem ,Global Value Toolkit“ ein Werkzeugkasten bereit, welcher über einen „Tool-Navigator“ Orientierung zu den diversen Tools des Nachhaltigkeitsmanagements bieten soll. Zudem enthält das Toolkit „Sektor-Profiles“, „Case Studies“ und „,Tool-Showcases \& Guides“ (15 führende Tools wurden gemeinsam mit multinationalen Konzernen verschiedener Sektoren ,getestet“, sind beispielhaft in ihrer Umsetzung einseh- und nachvollziehbar. Dies soll anderen Unternehmen ermöglichen besser und praxisbezogen einschätzen zu können, welches Tool das richtige für sie ist und durch welche(s) Tools sie am meisten für ihre Organisation und die Nachhaltigkeitsziele erreichen können) (vgl. Global Value Toolkit o.J.). 
Responsibility bezieht sich dabei auf zwei Dimensionen, zum einen auf eine interne, innerbetriebliche Dimension, die alle Unternehmensaktivitäten im Güter-/Dienstleistungserstellungsprozess umfasst. Es geht also um die Wertschöpfungskette des Unternehmens und die daraus resultierenden Auswirkungen auf Menschen bzw. die Gesellschaft (z.B. durch die Art der Einstellungspraktiken, Umgang mit Mitarbeitenden, Emissionen, Lieferkettenmanagement) (vgl. Riess 2010: 589). Die zweite Dimension wird dann tangiert, wenn „Unternehmen bisweilen wie Bürger [handeln], um konkrete Probleme ihres gesellschaftlichen Umfeldes zu lösen - etwa in sozialen oder kulturellen Projekten" (Riess 2010: 589) , auch ökologische Projekte können hier relevant sein. Im Kontext dieser Definition wird häufig auch von Corporate Citizenship gesprochen (vgl. Stoll 2009: 60f., Lang/Dresewski 2010: 401f., Habisch/Schwarz 2015: 114).

Wie bereits im Titel vermerkt, sollen sich die Ansätze zur Verankerung von CSR in Unternehmen sowohl auf profitwirtschaftliche als auch auf sozialwirtschaftliche Unternehmen beziehen (lassen). Deren maßgeblicher Unterschied ist typologisch betrachtet, dass sie aus unterschiedlichen Primärmotiven gegründet wurden bzw. existieren. Profitwirtschaftliche Unternehmen (große und kleine und mittlere Unternehmen, z.B. Konzerne wie Siemens, Bosch, Handwerkerbetriebe) streben primär nach - es liegt im Wort - Profit, nach Gewinn. Der genuine Fokus liegt auf ökonomischen, gewinnbezogenen Handlungslogiken bzw. -prinzipien wie Effizienz, Rationalität. Sozialwirtschaftliche Unternehmen (z.B. Einrichtungen der Diakonie, der Caritas, große und kleine sozialwirtschaftliche Einrichtungen, die keinem der Spitzenverbände der freien Wohlfahrtspflege zugehörig sind etc.) streben primär danach einem sozialen/gemeinnützigen Auftrag zu entsprechen ${ }^{5}$, sie umfassen die „Produzenten sozialer und gesundheitsbezogener Dienstleistungen“ (Grunwald 2014: 36). Der genuine Fokus liegt auf sozialen, auf Menschen bezogenen Handlungslogiken wie z.B. Förderung deren Autonomie, Achtung deren Personalität, Subsidiarität. Beiden ist aber gleich, dass eine stimmige Trias aus Ökologie, Soziales und Ökonomie entscheidend ist für den (langfristigen) Erfolg der

4 Die Lösung gesellschaftlicher bzw. spezifischer sozialer Probleme im Umfeld ist speziell für gemeinnützige Organisationen Usus bzw. Grund derer Existenz. Auf welche Art und Weise dies geschieht, auch mit welchem Rollenverständnis - auch in Abgrenzung bzw. Abstimmung mit anderen gesellschaftlichen Akteuren - muss auch in diesen Organisationen immer wieder zur Debatte gestellt werden. Genauso wie die Gestaltung des Dreiklanges aus Ökonomie - Ökologie - Sozialem in der/durch die Organisation. So formuliert Hofmacher (Malteser Werke) (2017: 36) im Kontext der gesellschaftlichen Verantwortung der Malteser Werke z.B.: es ist relativ weit verbreitet, „dass wir doch eigentlich immer schon zu den Guten gehören, weil wir an die Ränder der Gesellschaft gehen und dort helfen. Aber reicht das und ist das wirklich tragfähig, um in Zukunft Gutes zu tun?“"

$5 \mathrm{Zu}$ theoretischen Typen von Unternehmen, die in der Spanne zwischen Gemeinnützigkeit und Gewinnorientierung unterschieden werden können (vgl. z.B. Frischen/Lawaldt 2008: 3). Die Unterscheidung von weiteren Typen ist an dieser Stelle unerheblich bzw. die vorgestellten Ansätze können auch für diese „Zwischen-Typen“ herangezogen werden. 
Institution. Keine Organisation wird langfristig überleben können bzw. im Interesse ihrer diversen Stakeholder handeln und sich die gesellschaftliche Existenzberechtigung bewahren, wenn der Fokus entweder z.B. nur auf der eng verstandenen ökonomischen oder nur auf der eng verstandenen sozialen Dimension liegt. Die Beobachtung und Einschätzung (die einer weiteren Forschung bedarf), dass das Feld der gesellschaftlichen Verantwortung bzw. Nachhaltigkeit auch in sozialwirtschaftlichen Unternehmen in keiner Dimension zwangsläufig optimal - auch im Zusammenhang der Dimensionen nicht - bestellt ist, weist auch auf den Umstand hin, dass fehlende/rudimentäre CSR und Nachhaltigkeit nicht allein durch das derzeit (im profitwirtschaftlichen Kontext) vorherrschende Paradigma der unternehmerischen Gewinnmaximierung $^{6} \mathrm{zu}$ begründen ist. Anders formuliert: auch im Vordergrund stehende unternehmerische bzw. organisatorische geweinwohlorientierte Sachzielorientierung - statt Gewinnmaximierung - scheint kein Paradigma zu sein, was in einem Automatismus gesellschaftliche Verantwortung bzw. Nachhaltigkeit in „Reinform“ evoziert. Es liegt also, solange der Fehler noch „,irgendwo im komplexen System(en) liegt“ auch im Interesse beider „Unternehmensarten“ auf Ansätze zurückgreifen zu können, die es ermöglichen CSR im Unternehmen unter gegebenen Bedingungen und gegebenenfalls diese Bedingungen verändern, zu verankern.

Die Gründe dafür, Corporate Social Responsibility in Organisationen bzw. Unternehmen der Profit- bzw. Sozialwirtschaft zu verankern bzw. diese zum Leitkonzept der Unternehmen zu erklären, sind vielfältig. Bei näherer Betrachtung lassen sich diese in drei (auch interdependente) Komponenten fassen: So führen zum einen im engen Sinn a) geschäftliche Vorgaben und Rahmensetzungen (Business Case) dazu, dass die Wahrnehmung gesellschaftlicher Verantwortung in den Fokus rückt bzw. in diesem ist: hierzu können z.B. die CSRRichtlinie $^{7}$ bzw. das CSR-Richtlinien-Umsetzungsgesetz (RUG), Umweltschutzvorschriften, Fachkräfte-Mangel ${ }^{8}$, ein potenzieller Markt für verantwortlich produzierte Produkte bzw. erbrachte Dienstleistungen, Erwartungen an die Compliance der Organisation, geforderte Transparenz bezüglich be-

Damit wird auch deutlich, dass alleinig die Abkehr vom Prinzip der Gewinnmaximierung nicht ein vollständiger Lösungsansatz zur Verwirklichung organisationaler bzw. unternehmerische Verantwortung/Nachhaltigkeit sein wird.

7 Die sogenannte „CSR-Richtlinie“ des Europäischen Parlaments und des Rates wurde im November 2014 im Amtsblatt der Europäischen Union veröffentlicht. Mit der Richtlinie sind Unternehmen zukünftig gehalten, sich zu ihren Auswirkungen auf die Gesellschaft zu äußern. Dies soll über die jährliche Erstellung einer nichtfinanziellen Erklärung erfolgen, ,die mindestens Angaben zu Umwelt-, Sozial- und Arbeitnehmerbelangen, zur Achtung der Menschenrechte und zur Bekämpfung von Korruption und Bestechung enthält“ (aus Rn. 6: Richtlinie 2014|95|EU).

8 In diesem Kontext wird davon ausgegangen, dass ein gesellschaftlich verantwortlich handelndes Unternehmen attraktiver für potenzielle Arbeitnehmer*innen ist und daher der Arbeitnehmer*innengewinnung dienen kann. 
stimmter Geschäftsvorgänge (z.B. über Arbeitsbedingungen, Lieferketten, bezüglich der Mittelverwendung (z.B. von Spenden) in Nonprofit-Organisationen (NPOs)) gezählt werden. Auf Unternehmen heruntergebrochene bzw. auf diese ausgerichtet Vorgaben, Regelungen sind nicht selten Ausdruck bzw. Folgen eines breiteren b.) sozialen bzw. gesellschaftlichen Kontextes (Social Case), in dem die Unternehmen verortet sind und welcher auf diese wirkt bzw. auf den diese wirken (zum damit gegebenen Zusammenhang von außermarktlichen Themen und Business Case vgl. auch Schaltegger 2018: 360): so scheint die Komplexität gesellschaftlicher Herausforderungen nur noch bzw. besser zu bewältigen, wenn Staat, Wirtschaft und Zivilgesellschaft vereint an denselben arbeiten, sozial- und profitwirtschaftliche Unternehmen (und ggf. weitere) sich also gemeinsam an der Bewältigung gesellschaftlicher Herausforderung beteiligen. Teilhabe-Herausforderungen (diverser Zielgruppen wie z.B. bildungsbenachteiligte Jugendliche, Menschen mit Behinderung, geflüchtete Personen), soziale Spannungen (zwischen Menschen, die auch in Unternehmen hineinreichen/aus diesen hinausreichen), Klimawandel gehören z.B. den Bereich der systemübergreifenden Herausforderungen. Nicht zuletzt liegt der Antrieb für CSR und auf welche Art und Weise diese im und für das Unternehmen und die Gesellschaft gelebt wird, in einer persönlichen Komponente. Personen im/des Unternehmens bzw. der Organisation nehmen Business Case und Social Case auf ihre persönliche Art und Weise (Personal Case) zur Kenntnis, messen diesen ihre jeweilige persönliche Bedeutung zu (auch eingebettet in deren ethische und moralische Wertekontexte und Überzeugungen (z.B. Überzeugung der Notwendigkeit des ökologischen Schutzes der Welt; „Gefühl“ benachteiligte Menschen unterstützen zu müssen/wollen)) und entscheiden dann (im Ansatz) auf welche Art und Weise sie die Komponenten als ihr „CSRKonzept" im Unternehmen integrieren. Zusammengefasst sehen sich Unternehmen(svertreter*innen) der Herausforderung gegenüber:

- CSR-bezogene unternehmerische Vorgaben zu erfüllen (Business Case) bzw. CSR für „das Geschäft“ zu nutzen,

- sie bewegen sich als Unternehmer*in/Unternehmensvertreter*in und Bürger*in in der Gesellschaft und nehmen deren „Bedürfnisse“ und Rahmenbedingungen wahr (Social Case). Es stellt sich auch die Frage, wie „das Geschäft“ für CSR/Nachhaltigkeit nutzen soll/kann

- und sind dann als Person und in persona damit konfrontiert bzw. sehen es als ihre Aufgabe, unternehmerisch und gesellschaftlich wahrgenommene Handlungsaufforderungen so aufgreifen zu können, wie es auch der eigenen Person, dem eigenen Verständnis von Unternehmer*innentum, den eigenen Werten, Überzeugungen, der eigenen Auffassung von „richtigem" Handeln entspricht.

Nicht selten scheint sich damit eine Komplexität an Verantwortung und Handlungsaufforderungen zu ergeben, die kaum mehr zu bewältigen ist. CSR wird 
zur gleichermaßen unternehmerischen, sozialen bzw. gesellschaftlichen und persönlichen Managementherausforderung. Unter Umständen werden noch Möglichkeiten gesehen, an einzelnen Brandherden ,schnell“ und kurzfristig zu löschen, Handlungen (und Wirkungen) bleiben unsystematisch, richten sich „nach dem Wind“, es bleibt das Gefühl/der Druck etwas tun zu müssen etc.

Gerade auch in der Sozialwirtschaft scheint CSR bzw. Nachhaltigkeit noch nicht bzw. selten umfassend und systematisch eingebunden - bzw. CSR ist nicht selten für die sozialwirtschaftlichen Organisationen primär im Kontext der sozialen Kooperation mit Profitunternehmen ein Begriff (vgl. Hofmacher 2017: 36) - bzw. darstellbar zu sein: „Unternehmen werden grün, sozial und nachhaltig. Doch nur bei wenigen Trägern der Sozialwirtschaft ist die Umstellung auch messbar" (Eichmann 2016: 30). Zu einem Träger der exemplarisch aktiv in dieser „Umstellung“ ist, können die Malteser Werke (Migrationsarbeit, Jugend- und Suchthilfe, Träger von Schulen) gezählt werden. Das sozialwirtschaftliche Unternehmen stellt sich dich (strategische) Frage, worin die gesellschaftliche Verantwortung ihres Tuns und worin dessen Wirkung liegt (vgl. Hofmacher 2017: 36). Dies wird als ein Prozess betrachtet, der eine „Organisation regelrecht durchrütteln kann" (ebd.).

Managementsysteme, die es erlauben, CSR mit dem Unternehmen zu verweben, können (und sollten) ein Ansatz zur Bewältigung bzw. Gestaltung dieses „Durchrüttelns“ und des o.g. Komplexitätserlebens sein. Sie sollten dazu dienen können, CSR als „Wesenszug“ des Unternehmens zu begreifen, als „Charakter" der Geschäftstätigkeit. Das unternehmerische Geschehen muss so gestaltbar sein, dass die Facetten des unternehmerischen Handelns in ihrer Verbindung zur CSR stehen, normatives, strategisches und operatives Management in systematischem, automatischem (im Sinne von zwangsläufigem und unweigerlichem) und dialogischem Bezug zur CSR steht.

Neben der Frage, ob und inwiefern die im Folgenden vorgestellten Ansätze sowohl für die profit- als auch sozialwirtschaftliche Unternehmen stimmig sind (Erläuterungen hierzu s. Abschn. 2.2, 3.2), ist ebenfalls noch auf deren Eignung je nach Größe der Unternehmen (s. Abschn. 2.1, 3.1) einzugehen. Hier kann bereits an dieser Stelle festgehalten werden, dass die Ansätze für alle Unternehmensgrößen realisierbar sind. Keiner der beiden Ansätze ist so gestaltet,

Nach derzeitigem (Forschungs-)Stand ist dies nicht belegt. Der Umstand, dass Unternehmen der Sozialwirtschaft noch kaum eine Rolle im Kontext der Nachhaltigkeitsberichterstattung spielen, könnte aber hierfür ein Indiz sein (wohl wissend, dass nicht jede soziale Organisation mit Nachhaltigkeitsbericht diesen zum Ranking einreicht, s. z.B. den Nachhaltigkeitsbericht der Malteser Werke (MW 2013). So finden sich z.B. in der Nachhaltigkeits-Ranking-Liste 2011 (IWÖ/future) bezogen auf kleine und mittlere Unternehmen zwei Einrichtungen des Sozialwesens (Dienste für Menschen gGmbH und ,Regens Wagner Zell“ (keine Großunternehmen) (vgl. IWÖ 2012: 1f.), in den nachfolgenden Rankings der IWÖ von 2015 (vgl. Hoffmann/Dietsche/Westermann [u.a.] 2016: 16f.) und 2018 (vgl. Dietsche/Lautermann/Westermann 2019: 24 ff.) fanden weder bei den KMU noch bei den Großunternehmen sozialwirtschaftliche Unternehmen Eingang. 
dass es in der Natur des Ansatzes liegt z.B. ein KMU/KMO (kleine und mittlere Unternehmen bzw. Organisationen) zu überfordern bzw. dass er einer notwendigen Komplexität großer Unternehmen, sowohl in der Sozial- als auch der Profitwirtschaft, nicht gerecht werden könnte. Die stimmige Ausgestaltung und die notwendige Konsequenz in der Ausgestaltung und Umsetzung des CSR-Managementsystems liegt in der Hand der Unternehmen.

Die beiden folgenden Abschnitte 2 und 3 dienen der Erläuterung der beiden Ansätze bzw. Managementsysteme:

\section{Verankerung von CSR durch die Balanced Scorecard- Perspektiven(n)}

\subsection{Hintergrund des Managementsystems Balanced Scorecard}

Der Entstehungskontext des Managementinstruments (synonym „Managementsystem") Balanced Scorecard (ausgewogener Berichtsbogen) ist zunächst nicht direkt und ausdrücklich mit dem Konzept der CSR verbunden. Sie wurde Anfang der 90er Jahre vorrangig aus den gesehenen ,neuen“ Herausforderung in profitwirtschaftlichen Unternehmen heraus entwickelt. Ausgangslage von Kaplan/Norton (1997: 1ff.) ist dabei, dass Unternehmen wie auch das Umfeld, in dem diese agieren, heute derart komplex sind, dass sie nicht mehr rein durch finanzielle Ergebniskennzahlen gesteuert werden können. Das Umfeld der Unternehmen (dies gilt auch für sozialwirtschaftliche Unternehmen) - geprägt durch Globalisierung, notwendige Spezialisierung von Fähigkeiten, veränderte Kundenbeziehungen, Innovationsgedanken, Bedarf an lernenden und Wissen schaffenden Mitarbeiter*innen - erfordert es, auch die unternehmerischen Sachanlagen (z.B. Informationssysteme) und nicht physischen Vermögenswerte (z.B. intellektuelles Kapital) zu mobilisieren und zu verwerten (vgl. auch Stoll 2013: 78). Dazu leitet das Unternehmen seine Ziele (bzw. die einer Geschäftseinheit) von der Vision (bzw. Mission) und der Strategie des Unternehmens ab und entwickelt entsprechende Kennzahlen, mittels derer die Zielerreichung überprüft werden kann. Relevant sind dabei nicht nur finanzielle Ziele und Kennzahlen, sondern auch solche, die sich auf die Kunden/Stakeholder des Unternehmens, seine internen Geschäftsprozesse und das Lern- und Entwicklungspotenzial (der Mitarbeiter*innen/der Organisation) im Unternehmen beziehen (Kaplan/Norton 1997: 8f., 62ff.). Die Fragen, die hinter den Perspektiven stehen sind:

- Wie sollen wir gegenüber unseren Teilhabern/Stakeholdern auftreten um finanziellen/wirtschaftlichen Erfolg zu haben (Finanzperspektive); 
- wie sollen wir gegenüber unseren Kunden/Stakeholdern auftreten, um unsere Vision zu verwirklichen (Kunden/Stakeholderperspektive);

- in welchen Geschäftsprozessen müssen wir die Besten sein, um unsere Stakeholder/Kunden zu befriedigen (Interne Prozessperspektive);

- wie können wir unsere Veränderungs- und Wachstumspotenziale fördern, um unsere Vision zu verwirklichen (Lern- und Entwicklungsperspektive) (in Anlehnung an Kaplan/Norton 1997: 9).

Ein Aspekt der intendierten „Balance“ (Ausgewogenheit) der Scorecard kommt damit bereits zum Ausdruck, nämlich die Ausgewogenheit zwischen extern orientierte Zielen und Messgrößen für die externen Stakeholder (finanziell, kundenorientiert) und internen Zielen bzw. Messgrößen für kritische Prozesse, Innovation sowie Lernen und Wachstum. Hinzu kommt eine Ausgewogenheit zwischen Kennzahlen, die die Ergebnisse vergangener Tätigkeiten abbilden (Spätindikatoren, z.B. Gewinnzahlen) und Kennzahlen, die zukünftige Leistungen antreiben (Frühindikatoren, z.B. Kennzahlen zur Motivation oder Entwicklung der Mitarbeitenden). Gleichermaßen geht es um den ausgewogenen Einbezug von objektiven, leicht zu quantifizierenden Ergebniskennzahlen (z.B. Gewinn, Return on Investment), und subjektiven, urteilsabhängigen Leistungstreibern (z.B. Mitarbeitendenmotivation, Kunden-/Stakeholderzufriedenheit) (vgl. Kaplan/Norton 1997: 10, 144; Friedag/ Schmidt 2002: 65ff.).

Übergreifend ist die BSC als ein Managementinstrument zu versehen, das den organisatorischen Rahmen für alle Managementprozesse bildet. Sinn und Zweck der BSC ist es (vgl. Kaplan/Norton 1997: 11, vgl. auch Stoll 2013: 82) durch eine langfristige Verfolgung der Strategie des Unternehmens kritische Managementprozesse zu meistern und zwar bezüglich:

Der Klärung und dann dem Herunterbrechen von Vision und Strategie auf konkrete strategische Ziele:

Die aus der (z.B. nachhaltigkeitsorientieren) Vision abgeleitete Strategie wird in spezifische (aber nur in die wichtigsten) strategische Ziele übersetzt und die Ziele mit einer Messgrößen- bzw. Kennzahlen/Zielwert-Kombination verbunden. Die Ziele beziehen sich auf die (s.o.) Finanzen, die Kunden/Stakeholder, die internen Geschäftsprozesse (bestehende oder fehlende) und das Lernen für und die Entwicklung in der Organisation (vgl. Kaplan/Norton 1997: 11f., 142).

Der Kommunikation und Verknüpfung von strategischen Zielen und Maßnahmen:

Zur Kommunikation, welche strategischen Ziele für den Erfolg des Unternehmens angestrebt werden, werden die Ziele der BSC im gesamten Unternehmen verbreitet. Auf allen Managementebenen bzw. generell muss der Dialog gefördert werden, um Unterstützung zur Implementierung und Umsetzung der BSC zu erhalten. Durch das Verständnis der Mitarbeiter*innen für die Ziele, können deren Aktivitäten mit den Erfolgsfaktoren des gesamten Unternehmens verknüpft werden (vgl. Kaplan/Norton 1997:12f., 192ff.) 
Planung, Festlegung von Zielen und Abstimmung strategischer Initiativen: „Das Management" legt Ziele für drei bis fünf Jahre im Voraus fest. Die Ziele sollen dabei eine außerordentliche Leistung/Herausforderung für das Unternehmen/die Geschäftseinheit darstellen. Bei und nach Festlegung der Ziele ist auf deren (sich gegenseitig unterstützenden) Zusammenhang/Verknüpfung der Ziele zu achten. Um auf die angestrebten Zielzustände zu kommen, werden Initiativen entwickelt und umgesetzt. Die langfristige Planung über die BSC muss mit den jährlichen Maßnahmen verknüpft werden und Meilensteine für die verschiedenen Kennzahlen gesetzt werden (vgl. Kaplan/Norton 1997: 13f., 216ff.).

Verbesserung von strategischem Feedback und Lernen:

Durch die BSC erhält „das Management“ Rückmeldung zu den gewählten Strategien und die Richtigkeit der Hypothesen, auf denen die Strategien basieren. In verschiedenen Zeitspannen (z.B. monatlich, vierteljährlich) kann - über alle Ausführungsebenen hinweg - überprüft werden, ob die Ziele in den verschiedenen Perspektiven erreicht werden, es kann prognostiziert werden, ob die Zukunftserwartungen und die Gültigkeit der Strategie aufrechterhalten werden können. Es vollzieht sich somit ein strategischer Lernprozess, der mit der Visions- und Strategiefindung beginnt, Kommunikations- und Verknüpfungsprozesse sowie den Planungs-, Zielsetzungs- und Abstimmungsprozess umfasst und in einem (erneuten) Überdenken der Vision und Strategie resultiert (vgl. Kaplan/Norton 1997: 15ff., 192ff., vgl. auch Stoll 2013: 85f.).

Im Ablauf der Entwicklung der BSC lassen sich (nach Ackermann 2000: 39f.) zwei Standardmodelle unterscheiden. Das erste Modell orientiert sich an den Entscheidungsphasen, im ersten Schritt wird der BSC-Teamauftrag festgelegt, erfolgt die Team-Zusammenstellung und die Bestimmung des Arbeits- und Zeitplans. Im zweiten Schritt wird die Vision/Mission und Strategie des Unternehmens festgelegt. Im dritten Schritt werden für alle Perspektiven die Ziele festgelegt, dann erst alle Maßnahmen (vierter Schritt), dann im fünften Schritt alle Sollwerte (Zielvorgaben) um schließlich die Aktionsprogramme (6. Schritt) zu definieren um dann die erarbeiteten Inhalte zu präsentieren ( 7. Schritt).

Das zweite Vorgehen unterscheidet sich dahingehend, dass nicht zunächst in allen Perspektiven Ziele, dann erst Maßnahmen, dann Sollwerte etc. erarbeitet werden, sondern eine „Perspektive“ wird komplett bearbeitet, dann die nächste in Angriff genommen. Es kann davon ausgegangen werden, dass die Integration der BSC in den regulären Managementzyklus einen Zeithorizont von ca. 24 bis 36 Monaten umfasst. Abhängig von der Verfügbarkeit der beteiligten Personen, kann mit einer Entwicklungszeit für eine BSC (ggf. Auswahl der Organisationseinheit, Zieldefinitionen, Auswahl der Kennzahlen, Erstellung des Umsetzungsplanes) von ca. 16 Wochen gerechnet werden. 
Strategische Überlegungen und eine mehrperspektivische Betrachtungsweise des unternehmerischen Geschehens bzw. Teilaspekten desselben sind sowohl für Großunternehmen als auch KMU/KMO relevant. Entsprechend ist die Frage, ob eine Balanced Scorecard oder nicht, keine Frage der Größe einer Organisation (BSC für Gesamtorganisation) oder der Größe eines organisationalen Teilaspektes (z.B. BSC für eine Geschäftseinheit).

\subsection{Balanced Scorecard in Profit- und sozialwirtschaftlichen Unternehmen}

Bevor nun heruntergebrochen und dargelegt werden soll und kann, inwiefern die BSC maßgeblich für die Ausrichtung der CSR bzw. der Verankerung der CSR im profit- und sozialwirtschaftlichen Unternehmen sein kann, wird mit diesem Abschnitt betrachtet, ob und inwiefern die BSC bereits in ihren Grundlagen für beide „Unternehmensformen“ (profit-/sozialwirtschaftliche Organisationen) geeignet ist.

Der originäre bzw. der ursprünglich primäre Blick der „Begründer“ der Balanced Scorecard liegt auf der BSC für das profitwirtschaftliche Unternehmen. Allerdings verweisen die Autoren (vgl. Kaplan/Norton 1997: 173) bereits selbst darauf, dass die Optimierungsmöglichkeiten, die sich für staatliche und Nonprofit-Organisationen durch die BSC ergeben, noch weitaus höher sein dürften, als für profitwirtschaftliche Organisationen (da mit der Entwicklung und Umsetzung der BSC ein Denken und Festlegungen gefordert sind, die u.U. und teilweise von sozialwirtschaftlichen Organisationen bisher vernachlässigt wurden, z.B. explizite Zielformulierungen, Fragen der Erfolgs-/Wirkungsmessung). Außer Diskussion steht, dass die BSC beiden Unternehmensformen dazu dient, ihre Mission bzw. Vision zu verwirklichen. Die grundlegendste Unterscheidung/notwendige angepasste Betrachtungsweise bezieht sich auf die „Position“ der Finanzperspektive (vgl. Kaplan/Norton 1997: 178f., vgl. auch Stoll 2013: 94). In profitwirtschaftlichen Unternehmen soll die BSC final primär der Optimierung des Gewinns/dem Gewinnziel dienen. Damit steht doch, auch wenn es um die Balance der Perspektiven geht, in profitwirtschaftlichen Unternehmen mehr oder weniger ,heimlich“ und bildlich gesprochen die Finanzperspektive an der „Spitze“ der Scorecard (s. Abb. 1).

$\mathrm{Ob}$ ihres gesellschaftlichen Auftrages liegt hingegen der Sinn der BSC in sozialwirtschaftlichen Organisationen in der optimierten Erreichung der Sachziele, d.h. die Orientierung an Bedürfnissen und Bedarfen der Zielgruppen und weiterer Stakeholder sozialwirtschaftlicher Organisationen. Daher ist die Kunden bzw. Stakeholder-Perspektive hier an der „Spitze“ zu sehen (vgl. Kaplan/Norton 2001: 120f., vgl. auch Haddad 1998: 59ff., Berens/Karlowitsch/ Mertes 2000: 26ff., Gmür 2000: 193ff., Stoll 2013: 95ff.). 
Abbildung 1: Die BSC und ihre Perspektiven in profit- und sozialwirtschaftlichen Organisationen

\section{Profitwirtschaftliche Organisation}

Kunden-/
Stakeholder-
Perspektive

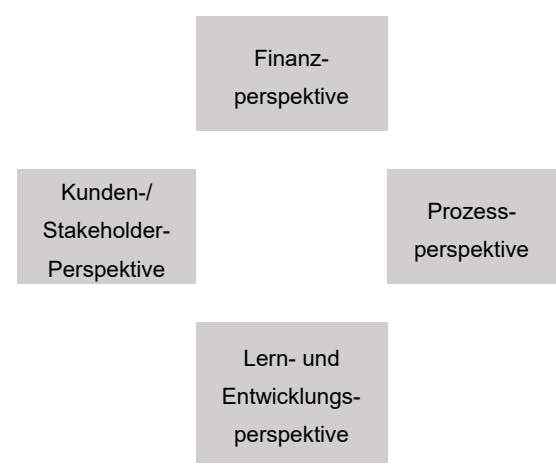

\section{Sozialwirtschaftliche Organisation}

\section{Kunden- $/$ \\ Stakeholder- \\ Perspektive}

Finanz-

perspektive

Quelle: eigene Darstellung

\subsection{Verankerung der CSR im Management und Unternehmen}

Prinzipiell kann die gesellschaftliche Verantwortung der Unternehmen auf drei Weisen in eine/die Balanced Scorecard integriert werden bzw. diese eine gesellschaftlich verantwortliche Ausrichtung des Unternehmens sicherstellen:

2.3.1 (Strategische) Ziele zur CSR sind Bestandteil der Balanced Scorecard für die Gesamtorganisation

Wie ein Unternehmen etwa feststellen kann, dass es für es selbst strategisch relevant ist seine Fehlerquote im Produktionsprozess zu optimieren, kann es ebenso sein, dass festgestellt wird, dass es strategisch bedeutsam ist, den Energieverbrauch (offensichtlicher Zusammenhang zu CSR) z.B. im Produktionsprozess, zu senken. Entsprechende Ziele, Zielvorgaben, Kennzahlen, Maßnahmen zur Realisierung werden deshalb dahingehend formuliert. Bei diesem Modell gilt: Die gesamte Geschäftstätigkeit, das gesamte unternehmerische Wirken wird unter vier Perspektiven betrachtet. Das ein Unternehmen dabei auch aus den unterschiedlichsten Gründen heraus per se in einem Zusammenhang mit den internen und externen gesellschaftlichen bzw. sozialen Wirkungen zu betrachten ist/betrachtet werden sollte, fließen zwangsläufig CSR-relevante Ziele etc. in die Perspektiven der BSC ein. 
Diese Zugangsweise entspricht am ehesten dem Verständnis eines Unternehmens, das sich generell, als Unternehmen mit der kompletten Wertschöpfungskette als sozialverantwortlich handelndes und gesellschaftliche wirkendes Unternehmen betrachtet und sich entsprechend aufstellen möchte.

Möchte ein Unternehmen aus bestimmten Gründen die „CSR-Perspektive“ nach außen deutlicher erkennbar werden lassen, ist es z.B. denkbar, die vier „typischen“ Perspektiven um eine „CSR-Perspektive“ zu erweitern:

\subsubsection{CSR durch eine 5. Perspektive}

„Es gibt keine mathematische Formel, die beweist, daß vier Perspektiven notwendig und ausreichend sind“" (Kaplan/Norton 1997: 33). So ist es also generell denkbar, dass die „typische“ vier-perspektivische BSC speziell um eine CSR-Perspektive erweitert wird (vgl. Stoll 2004: 33). Bei diesem Modell gilt also: CSR wird nicht in direktem Zusammenhang zur Bedeutung für die Stakeholder der Organisation betrachtet, es wird nicht nach der direkten nachhaltigen Gestaltung von Prozessen ,gefragt“ etc., sondern „reine CSR-Ziele“ unterschiedlichster Art in einer separaten Perspektive formuliert. Suboptimal herbei ist, dass CSR damit aus der „Kette der kausalen Zusammenhänge“ (Kaplan/Norton 1997: 34) zwischen den Perspektiven herausgenommen wird. Es entsteht ein isoliertes Paket an Kennzahlen (vgl. ebd.) für Corporate Social Responsibility. Ist aber eine zusätzliche CSR-Perspektive, ein Medium des Einstiegs in die folgende generelle Ausrichtung des Unternehmens am „Leitkonzept CSR“, dann ist eine solche fünfte Perspektive positiv zu bewerten. Genauso, wenn damit das Unternehmen zunächst zum Ausdruck bringen möchte, dass dieser Perspektive auf das Unternehmen (nun) auch eine Bedeutung kommen soll. So ist z.B. denkbar, dass erst die „zusätzlichen“ Überlegungen zur CSR-Perspektive die Zusammenhänge zu allen anderen Perspektiven und damit zum Gesamtunternehmen deutlich werden lassen und in der weiteren Entwicklung des Unternehmens CSR dann integrierter Teil der Kunden-, Finanz...-Perspektive wird. Eine ähnliche Betonung, aber auch eine von der ,sonstigen“" Geschäftsstrategie abgrenzte Betrachtung, erhält CSR über eine sogenannte CSR- oder Nachhaltigkeits-Balanced Scorecard:

\subsubsection{Betrachtung von CSR unter vier Perspektiven}

Bei dem Modell der CSR- bzw. Nachhaltigkeits-BSC gilt: CSR (nicht die Organisation/die Organisationstätigkeit) an sich wird unter 4 Perspektiven betrachtet (vgl. Stoll 2004: 3, vgl. Lotter/Braun 2016: 107ff., Lotter/Braun 2017: 66ff.) Mit diesem Modell wird etwa unter der Kunden-/Stakeholder-Perspektive überlegt, was Kunden bzw. Stakeholder in Sachen CSR vom Unternehmen erwarten, entsprechend formuliert das Unternehmen - auch aus einem eigenen strategischen Interesse heraus - Ziele um diesen Erwartungen entsprechen zu 
können. So kann es z.B. strategisch bedeutsam sein, dass die Mitarbeiter*innen das Unternehmen als einen vorbildlich ,gesundheitsfördernden“ Arbeitgeber wahrnehmen (Ziel), erfasst wird die Erreichung des Ziels z.B. über die Kennzahl der „Mitarbeiter*innenzufriedenheit, Mitarbeiter*innenbindung, -motivation, Krankenstand...“. Das Unternehmen hat entsprechende Maßnahmen zur Zielerreichung (z.B. Erfassung Ist-Situation, Bedarfsabfrage, Entwicklung eines Gesundheitsprogramms) zu veranlassen. Unter der Prozessperspektive fragt sich das Unternehmen, welche Prozesse auf welche Weise zentral sind, um Stakeholder bei der Umsetzung nachhaltigen Handelns zufriedenzustellen. So kann es sinnvoll sein in einen bewussten sozialen Dialog mit Stakeholdern (Kunden, Lieferanten, Mitarbeiter*innen) (Ziel) zu treten/stehen (bezogen auf das vorliegende Beispiel kann ein solcher Dialog mit den Mitarbeiter*innen zu sinnigen Inhalten des Gesundheitsprogrammes führen). Kennzahl zur Überprüfung der Zielerreichung kann das Vorhandensein oder Nichtvorhandensein eines solchen Dialogs (oder bestimmter Formate) sein. Das Unternehmen muss zur Ermöglichung z.B. eine Stakeholder-Analyse vornehmen, Dialogformat/e festlegen, Dialoge implementieren... (Maßnahmen). Unter der Finanzperspektive betrachtet das Unternehmen, welche wirtschaftlichen Konsequenzen CSR für das Unternehmen haben kann und soll. So kann es z.B. Ziel sein, dass eventuelle Mehraufwendungen für verantwortliches handeln durch Effekte aus CSR gedeckt sein sollen (Ziel). In diesem Kontext relevante Kennzahlen können z.B. sein der „Mehraufwand“ (z.B. für die Etablierung eines Gesundheitsprogramms), „Einsparungen“ (z.B. bei „Fluktuationskosten“, „Krankheitstagen“). Unter der Lern- und Entwicklungsperspektive bzw. Innovationsperspektive stellt sich die Frage, wie im Unternehmen das Lern- und Entwicklungspotenzial des Unternehmens und dessen Mitarbeiter*innen zugunsten CSR gefördert bzw. genutzt werden kann. So kann etwa ein strategisch bedeutsames Ziel im Sinne der Verankerung der CSR im Unternehmen sein, dass die Mitarbeiter*innen des sozial- oder profitwirtschaftlichen Unternehmens selbst Akteur*innen sozial/gesellschaftlich verantwortlichen Handelns im Unternehmen sind bzw. werden. Erfasst werden kann die Zielerreichung z.B. über die Kennzahl „Anzahl der Verbesserungsvorschläge“ (zugunsten CSR, z.B. bezüglich ökologischer, sozialer Handlungsweisen im Unternehmen etc.). Als Maßnahme ist dafür z.B. notwendig, dass ein entsprechendes - nicht notwendiger Weise komplexes - Vorschlagswesen im Unternehmen etabliert wird. Wichtig ist, dass die einzelnen Perspektiven, die darin enthaltenen Ziele und Kennzahlen in einem kausalen Ursache-Wirkungs-Zusammenhang stehen. Die einzelnen Inhalte in den und über die Perspektiven hinweg dürfen sich nicht gegenseitig torpedieren.

Bei allen Varianten ( $a, b, c)$ können jeweils gleiche Ziele zum gesellschaftlich verantwortlichen Handeln des Unternehmens einfließen. So kann z.B. sowohl im Modell a, als auch Modell c, das Ziel, ,sich als gesundheitsfördernder Arbeitgeber aufzustellen und als solcher wahrgenommen zu werden" in der 
Stakeholder-Perspektive verortet sein, im Modell b kann das Ziel Eingang in die „CSR-Perspektive“ finden. Letztlich sagt die Wahl ob Modell a, b oder c „nur" etwas darüber aus, wie man (zum Zeitpunkt der Erarbeitung der entsprechenden Variante) als Unternehmen die Verbindung von Unternehmenstätigkeit und CSR betrachtet und wahrnimmt und signalisiert dies auch entsprechend den internen und externen Stakeholdern. Die Grenzen zwischen den Modellen können damit auch fließend sein, so kann z.B. eine im/für das Unternehmen entwickelte CSR-Balanced Scorecard - entwickelt zum Zwecke der Annäherung an CSR und als Möglichkeit einer überschaubaren ersten Schritte - dazu führen, dass über die Zeit hinweg, das Modell a Anwendung findet.

Festgehalten werden kann generell bei dem Balanced Scorecard-orientierten Ansatz (unabhängig davon, welches Modell), dass über die Verbindung von BSC und CSR, die grundlege Ausgangslage bzw. Kontext von CSR die Geschäftstätigkeit des Unternehmens ist, diese wird auf ihre Verbindung bezüglich CSR überprüft und in entsprechende Verbindung gesetzt, abhängig vom Modell ist diese Verbindung stärker oder geringer. Die BSC - in ihrer Funktion als strategisches Managementinstrument - wird eingesetzt mit dem Gedanken nachhaltigem Handeln im Unternehmen Ausdruck verleihen zu können. Dabei kann es insbesondere bei Modell a sein, dass ganz grundlegend eine ,natürliche Verbindung“" zwischen Geschäftstätigkeit und dem dreidimensionalen nachhaltigen Handeln gesehen wird, dass (strategisches) Management nachhaltiges Handeln inhäriert. Sowohl als Vorteil als auch als Nachteil, kann es empfunden werden, dass die BSC an sich keinerlei Vorgabe bzw. Orientierung gibt, keine Voreinordnung bereitstellt, was als sozialverantwortliches bzw. gesellschaftlich verantwortliches Handeln gilt, welche Kernthemen mit welchen Handlungsfeldern (s.u.) in welchem Maße aufzunehmen sind. Hier gilt es sich anderweitig Informationen zu beschaffen bzw. sich im Rahmen der BSC und deren Umsetzung konkretisierender CSR-Instrumente zu bedienen (dies kann z.B. durch Integration von EMASplus geschehen).

\section{Verankerung von CSR durch EMAS ${ }^{\text {plus }}$}

\subsection{Hintergrund des Managementsystems EMASplus}

Während die Balanced Scorecard ein allgemeines, strategisches Managementinstrument ist, über welches CSR integriert wird/werden kann, ist EMASplus 
per se als ganzheitliches Nachhaltigkeitsmanagementsystem entwickelt worden ${ }^{10}$ und soll das Handeln einer Organisation konsequent darauf ausrichten „,ihre ökonomischen, ökologischen und sozialen Wirkungen systematisch zu bewerten und kontinuierlich zu optimieren" (kate e.V. 2012: 3). Die Orientierung am Gemeinwohl wird als legitimes Ziel in das Organisationshandeln einbezogen (ebd.). EMASplus ist ein - zertifizierbares, die DIN EN 14001-2015 einschließendes - System für ein Nachhaltigkeitsmanagement auf der Basis des „Gemeinschaftssystem für das freiwillige Umweltmanagement und die Umweltbetriebsprüfung" EMAS (Eco Management und Audit Scheme) und der ISO 26000"11 (vgl. kate e.V. 2012: 3). Die ISO 26000 (DIN 2011: 20) versteht dabei gesellschaftliche Verantwortung übergreifend als den Willen

„einer Organisation soziale und umweltbezogene Überlegungen in ihre Entscheidungsfindung einzubeziehen und Rechenschaft über die Auswirkungen ihrer Entscheidungen und Aktivitäten auf Gesellschaft und Umwelt abzulegen. Dies setzt sowohl transparentes als auch ethisches Verhalten voraus, das zu einer nachhaltigen Entwicklung beiträgt, anwendbares Recht einhält und im Einklang mit internationalen Verhaltensstandards steht. Es bedeutet auch, dass gesellschaftliche Verantwortung in die gesamte Organisation integriert ist, in den Beziehungen der Organisation gelebt wird und die Interessen der Anspruchsgruppen berücksichtigt."

EMASplus ist, wie auch die BSC, zunächst branchenunabhängig, lässt sich aber branchenspezifisch ausgestalten und ist unabhängig von der Größe des Unternehmens bzw. der Organisation (vgl. kate e.V. 2012: 3). ISO 26000 hat zum Ziel allen Arten von Organisationen, u.a. unabhängig von ihrer Größe, von Nutzen zu sein und geht davon aus, dass CSR in kleine und mittlere Organisationen (Mikroorganisationen), ,praktisch, einfach und kosteneffizient integriert werden" kann und nicht komplex oder teuer sein muss (DIN 2011: $8 \mathrm{u}$. 23). Vielmehr sollen sie aufgrund ihrer geringen Größe und ,ihrem Potential, flexibler und innovativer zu sein“, besonders gute Möglichkeiten haben gesellschaftliche Verantwortung wahrzunehmen. Ein flexibles Management der Organisation, der engere Kontakt in und mit lokalen Gemeinschaften, der direkte Kontakt und Einfluss der Führung auf die Aktivitäten der Organisation werden hier als begünstigend gesehen (a.a.O.: 23; zu den generellen „Trümpfen“ der KMO im Kontext CSR vgl. auch Stoll 2009: 163 ff.).

10 Finanziert und entwickelt im Rahmen eines EU Projektes durch die Stuttgarter Kontaktstelle für Umwelt und Entwicklung e.V. (kate) und Projektpartner und war/ist zu verstehen als eine „Empfehlung an die Europäische Kommission zur Weiterentwicklung von EMAS in Richtung eines Nachhaltigkeitsmanagements“"(vgl. UGA 2006: 1, vgl. kate e.V. o. J.).

11 Auch die ISO 26000 enthält den Bereich Umwelt, dabei wird von Seiten des Umweltgutachterausschusses (UGA) davon ausgegangen, dass „EMAS-Organisationen mit ihrem Managementsystem Strukturen und Handlungsgrundsätze etablieren sowie Umweltleistungen erbringen, die weite Teile der nur unverbindlichen Empfehlungen und Anregungen der ISO 26000 konkret in die Praxis umsetzen“(UGA 2012: 2). 
Die Kernthemen und Handlungsfelder gesellschaftlicher Verantwortung, auf die sich EMASplus bezieht, sind die der ISO 26000. Hierzu gehören die Kernthemen:

- Organisationsführung, sowohl als „Kernthema“ gesellschaftlicher Verantwortung als auch als „Instrument“, über das die Organisation befähigt wird, bezüglich der anderen Kernthemen verantwortlich zu handeln. „Handlungsfelder“ sind die Prozesse, Systeme, Strukturen und andere Mechanismen der Organisation, welche die Umsetzung der Grundsätze und Ansätze gesellschaftlicher Verantwortung ermöglichen und fördern.

- Menschenrechte, mit Handlungsfeldern wie „Menschenrechte in kritischen Situationen“, „Diskriminierung und schutzbedürftige Gruppen“ und ,grundlegende Prinzipien und Rechte bei der Arbeit";

- Arbeitspraktiken, mit Handlungsfeldern wie „Arbeitsbedingungen und Sozialschutz“, „Gesundheit und Sicherheit am Arbeitsplatz“, „Menschliche Entwicklung und Schulung am Arbeitsplatz";

- Umwelt, mit Handlungsfeldern wie „Vermeidung der Umweltbelastung“, Nachhaltige Nutzung von Ressourcen, „Umweltschutz, Artenvielfalt und Wiederherstellung natürlicher Lebensräume;

- Faire Betriebs- und Geschäftspraktiken, mit Handlungsfeldern wie „Korruptionsbekämpfung“, „Verantwortungsbewusste politische Mitwirkung“, „Fairer Wettbewerb“,

- Konsumentenanliegen, mit „Handlungsfeldern“ wie „Nachhaltiger Konsum“, Schutz und Vertraulichkeit von Kundendaten, Verbraucherbildung und Sensibilisierung" sowie das Kernthema

- Einbindung und Entwicklung der Gemeinschaft, mit Handlungsfelder wie „Einbindung der Gemeinschaft“, „Schaffung von Wohlstand und Einkommen“, Investition zugunsten des Gemeinwohls“ (vgl. DIN 2011: 10f.; 38ff.).

Als Resultat eines Multi-Stakeholder-Ansatzes ${ }^{12}$, entsprechen die in der internationalen Norm aufgenommenen Kernthemen und Handlungsfelder dabei nach DIN (2011: 19) „,der gegenwärtigen Auffassung von bewährter Praxis“, Auffassungen, die sich auch in der Zukunft ändern können (wie die Aspekte gesellschaftlicher Verantwortung generell zu einem bestimmten Zeitpunkt die

12 Fachleute, aus mehr als 90 Ländern und 40 breit aufgestellten internationalen oder regionalen Organisationen, die in verschiedenen Bereichen gesellschaftlicher Verantwortung tätig sind, umfassend (vgl. DIN 2011: 5). 
Erwartungen der Gesellschaft spiegeln und damit Veränderungen unterliegen). ${ }^{13}$ Der Ansatz der ISO 26000, dass sowohl die Einhaltung rechtsverbindlicher Verpflichtungen als auch von Verpflichtungen, die sich aus ,allgemein gültigen ethischen und anderen Werten" (DIN 2011: 20) ergeben, in die Wahrnehmung gesellschaftlicher Verantwortung einfließen ${ }^{14}$, zeigt sich in den diversen aufgelisteten Handlungsfeldern (vgl. auch a.a.O.: 14).

Mit Hilfe dieser Kernthemen und Handlungsfelder soll die Organisation ihre prioritären Verantwortungsbereiche und für sie relevanten Themen und damit verbundenen Verbesserungspotenziale identifizieren (vgl. kate e.V. 2012: 4). Dabei wird auf eine „,neue Verantwortungs- und Dialogkultur" im Rahmen des Managementsystems gesetzt. Davon ausgehend, dass ein ethisches Wirtschaften, die Orientierung am Gemeinwohl und Gerechtigkeit, konträr zu ökonomischen Zielen der Organisation bzw. zu bestimmten Stakeholder-Interessen stehen kann, sollen gerade auch die Zielkonflikte transparent gemacht werden und im Rahmen einer seriösen Prüfung entschieden werden, welche diesbezüglichen Aspekte inwiefern in Managemententscheidungen Berücksichtigung finden. Dabei werden auch insbesondere die Beschäftigten als wichtige Stakeholder (Anspruchsgruppe) des Unternehmers verstanden ohne die kein nachhaltiger Erfolg möglich ist. Sie sollen aktiv am Nachhaltigkeitsprozess partizipieren, deren diesbezügliche Lernbereitschaft und Innovationskompetenz gefördert werden (vgl. a.a.O.: 4 f.). Bestandteile der Zertifizierung $^{15}$ nach EMASplus sind die Validierung nach EMAS, die Zertifizierung nach DIN EN ISO 14001-2015 ${ }^{16}$ und die Konformität zur ISO 26000, nachgewiesen durch einen Nachhaltigkeitsbericht (EMASplus o.J.). Zertifiziert wird durch unabhängige Dritte (zugelassene Umweltgutachter*innen mit $\mathrm{Zu}-$ satzqualifikation für EMASplus), basierend auf der EMAS-Verordnung.

13 EMASplus setzt an den Kernthemen an, rekurriert nicht explizit auf die in der Norm den Kernthemen bzw. der gesellschaftlichen Verantwortung zugrunde gelegten Grundsätze (Rechenschaftspflicht, Transparenz, Ethisches Verhalten, Achtung der Interessen der Anspruchsgruppen, Gesetztestreue, Achtung Internationaler Verhaltensstandard, Achtung der Menschenrechte) (vgl. DIN 2011: 12, 25ff.). Nach Auffassung des DIN (2011: 25) sollten Organisationen, die gesellschaftliche Verantwortung wahrnehmen, diese Grundsätze (und Grundsätze, die für die Kernthemen formuliert werden) achten. Zur (fehlenden/nicht klaren) Abgrenzung der Grundsätze von den Kernthemen (z.B. ist die Rede sowohl vom „Grundsatz“ als auch „Kernthema“ Menschenrechte), vgl. (in diesem Band) Rusche 2019: 5f.).

14 Zur Problematisierung der Unterscheidung, Gleichsetzung bzw. von Vermengung von juristischen und ethisch-moralischen Verpflichtungen und Implikation im Kontext von CSR in unterschiedlichster Hinsicht (vgl. z.B. in diesem Band Rusche 2019: 6f; Nowrot 2018: 218ff.).

15 Für die Zertifizierung fallen typische Beratungs- und Prüfkosten an.

16 EMAS geht über die ISO 14001 hinaus. ISO 14001 zielt auf die Optimierung des Managementsystems, EMAS auf eine - über gesetzliche Anforderungen hinausgehende - kontinuierliche Verbesserung der Umweltleistungen. Im weiteren vorteilig gesehen, wird die unabhängige Bewertung der Umweltleistung durch einen staatlich zugelassenen Umweltgutachter (vgl. Geschäftsstelle des Umweltgutachterausschusses 2018: 2). 


\subsection{EMASplus in profit- und sozialwirtschaftlichen Unternehmen}

Bereits die beiden richtungsgebenden Elemente von EMASplus - das Umweltmanagementsystem EMAS und der internationale Standard ISO 26000 - verweisen beide darauf, dass ,jedwede " Organisationen Bezugsorganisationen sind. So formuliert der UGA, dass Organisationen jeder Art mit dem EU-Label ausgezeichnet werden können, wenn sie die strengen Anforderungen der EMAS-Verordnung erfüllen (vgl. UGA o.J. $b$ ). Hierzu gehören, neben zahlreichen branchenunterschiedlichen Unternehmen der Profitwirtschaft, z.B. auch öffentliche Einrichtungen wie Behörden, Hochschulen und Organisationen der Sozialwirtschaft (so sind z.B. Einrichtungen der Diakonie, AWO, Kinderschutzbund, DRK EMAS-zertifiziert). Obwohl unter Umständen auf einen schnellen Blick irritierend, dass ,auch“ sozialwirtschaftliche Organisationen mit ihren per se gegebenen primär sozialen Zielen, als die „guten Organisationen“ explizit unter der „Perspektive“ der Wahrnehmung von gesellschaftlicher bzw. sozialer Verantwortung zu betrachten sind, erklärt die ISO z.B.: „Die Auffassung, dass alle Arten von Organisationen, und nicht nur privatwirtschaftliche, gesellschaftliche Verantwortung wahrnehmen sollte, folgt aus der Erkenntnis, dass alle Organisationen eine Verantwortung haben, zu einer nachhaltigen Entwicklung beizutragen." (DIN 2011: 18). Denkbar, aber in diesem Zusammenhang nicht belegt, ist, dass es spezifisch für sozial- bzw. profitwirtschaftliche Unternehmen Aspekte/Handlungsfelder gibt, in denen diese jeweils typischerweise bereits mehr verantwortliche Wahrnehmungs- und Handlungskompetenz aufweisen und jeweils andere Aspekte, die im Rahmen der interdependenten Trias „Ökonomie - Ökologie - Soziales“ neu/anders justiert werden müssen oder können. Entsprechend sind es auch sowohl Unternehmen der Profit- als auch der Sozialwirtschaft, welche ihre gesellschaftliche Verantwortung nach EMASplus wahrnehmen (können). Organisationen, die bereits EMASplus zertifiziert sind, sind z.B. die Hipp Gruppe (Babynahrung), die Katholische Universität Eichstädt-Ingolstadt, die gemeinnützige „Dienste für Menschen GbmH“"(Altenpflege).

\subsection{Verankerung der CSR im Management und Unternehmen}

Die Verankerung des Leitkonzeptes „CSR“ im Unternehmen erfolgt bei EMASplus über sechs Managementelemente (Dauer des Einführungsprozesses i.d.R. zwischen 8 und 12 Monaten). Hierzu gehören die Elemente:

- Leitbild: Systematische und interdependente Integration sozialer, ökologischer und ökonomischer Aspekte in das Organisationsleitbild.

- Nachhaltigkeitsprüfung: als Bestandaufnahme und Bewertung der Nachhaltigkeitswirkungen des Unternehmens. 
- Verbesserungsprogramm: das sich aus dem Leitbild und der Nachhaltigkeitsprüfung ergibt.

- Managementsystem: integriertes Managementsystem im Sinne einer Organisationsführung, durch welche die Nachhaltigkeitsaspekte in Struktur und Abläufen, in Schulung und Kommunikation der Organisation verankert werden.

- Monitoring: regelmäßig mit internem und externem Audit.

- Nachhaltigkeitsbericht: standardisiert (vgl. kate e.V. 2012: 6).

Im Detail ist bezüglich des Leitbildes ,im Zusammenwirken zwischen Leitung und Mitarbeitenden und unter Berücksichtigung der legitimen Interessen der relevanten Anspruchsgruppen“ (kate e.V. 2012: 6) ein verbindliches und zu lebendes Leitbild ${ }^{17}$ festzulegen, welches letztlich allen Mitarbeitenden und der Öffentlichkeit bekannt sein muss. In diesem soll deutlich werden, in welcher Verbindung das „Kerngeschäft“ zu Aspekten der Nachhaltigkeit bzw. der gesellschaftlichen Verantwortung steht, die grundlegende Verpflichtung zur Einhaltung relevanter Gesetze, Vorschritten etc. soll formuliert werden. Aus dem Leitbild müssen Einzelziele zur Nachhaltigkeit und Indikatoren zur Überprüfung der Erreichung ableitbar sein, die zirkulär auf eine stetige Verbesserung ausgerichtet sind. Im Rahmen der Nachhaltigkeitsprüfung, die der Ermittlung relevanter Nachhaltigkeitsaspekte dient, erfolgt erstens eine „Bestandsaufnahme“. Diese bezieht sich auf eine Mitarbeitendenbefragung, die Stärken/Schwächen, Chancen/Risiken der Organisation, die Stakeholder (-analyse), die Form des Dialoges mit den Stakeholdern, die ökonomischen, ökologischen und sozialen Leistungsindikatoren, den Grad der Nachhaltigkeitsintegration im Kerngeschäft (in Strategien, Produkten, Kommunikation, als Wettbewerbsfaktor) und umfasst eine Prüfung der Auswirkungen der Organisation auf die Gesellschaft im Abgleich mit den Kernthemen der ISO 26000. Zweitens ist eine „Bewertung“ vorzunehmen, welche der Nachhaltigkeitsaspekte wesentliche direkte und indirekte Auswirkungen haben und inwiefern diese vom Unternehmen beeinflussbar sind. Entsprechend sind durch das Unternehmen Prioritäten zu setzen. Die drittens zu dokumentierenden Ergebnisse („Dokumentation“) sind den Mitarbeitenden, ggf. auch der Öffentlichkeit, zu kommunizieren (vgl. kate e.V. 2012: 7f.). Das Verbesserungsprogramm dient dann der aus dem Leitbild und der Nachhaltigkeitsprüfung resultierenden Entwicklung von Zielen, der Ableitung von messbaren Indikatoren, Festlegung

17 Auch wenn Formulierungen (z.B. im Kontext der Definition von Meilensteinen im Nachhaltigkeitsmanagement: „Verabschiedetes Leitbild zur Nachhaltigkeit liegt vor“ (kate e.V. 2012: 6)) in der Richtlinie zum Nachhaltigkeitsmanagementsystem EMASplus dahingehend interpretiert werden können, das es unterschiedliche Leitbilder in der Organisation gibt/geben könnte, ist fraglich inwiefern es sinnvoll ist, als Organisation ein Organisationsleitbild und ein Nachhaltigkeitsleitbild zu entwickeln. Das Organisationsleitbild sollte den Nachhaltigkeitsgedanken/CSR mit einbeziehen, entsprechend ggf. das Organisationsleitbild angepasst oder ergänzt werden. 
von Verantwortlichkeiten, sowie Mitteln und Zeiträumen. Zur Implementierung des Nachhaltigkeitsmanagementsystems soll die Leitung der Organisation ein Leitungsmitglied bzw. eine*n Nachhaltigkeitsmanagementbeauftragte*n benennen. Durch diese*n gilt es sicherzustellen, dass die für EMASplus notwendigen erforderlichen Prozesse und Strukturen implementiert (inkl. bezüglich Notfallvorsorge und Umgang mit Risikosituationen) und aktiv aufrechterhalten werden. In der EMASplus-Richtlinie wird dabei besonders das notwendige Prozessmanagement, damit die Prozessqualität des Nachhaltigkeitsmanagementsystems, betont (Ermittlung und Darstellung der Prozesse über eine Prozesslandschaft ${ }^{18}$ ) und die Steuerung derselben (vgl. kate e.V. 2012: 9). Der Verweis, dass die Leitung, die für das Nachhaltigkeitsmanagementsystem (NMS) benötigten Mittel bereitstellen muss (erforderliches Personal, spezielle Fähigkeiten, Technologien und Finanzmittel, vgl. a.a.O.: 8), kann als Fokus auf die notwenige Strukturqualität des NMS verstanden werden. Bezüglich der Ergebnisqualität bzw. des wirkungsorientierten Controllings (s. auch unten „Monitoring“) werden regelmäße Berichte zur Wirksamkeit des Nachhaltigkeitsmanagements vorgesehen. Um die Zielerreichung des Verbesserungsprogramms zu unterstützen, werden Prozessbeschreibungen, Durchführungsanweisungen und sonstige Dokumente zum Managementsystem in einem „Managementhandbuch“" zusammengeführt, dessen Dokumente aktiv gelenkt. Neben der Zuschreibung von Verantwortung an ein Leitungsmitglied bzw. eine*n Nachhaltigkeitsmanagementbeauftragte* $n$ wird begleitend die Gründung bzw. Aktivität eines Nachhaltigkeitsrates empfohlen, dieser soll unterstützend das Managementsystem betreuen, weiterentwickeln, kommunizieren und dessen Wirkung stetig verbessern.

Teil des Managementsystems ist es auch Kommunikation zu betreiben (intern zum Zwecke der Beteiligung verschiedener Organisations-Ebenen/-Funktionen), den Schulungs- und Informationsbedarf zu relevanten Themen zu ermitteln und entsprechende Angebote zur Weiterbildung zu ermöglichen, sowie ein Stakeholder-Management zu etablieren (mit Regeln zur Auswahl relevanter Stakeholder-Gruppen, Stakeholder-Dialoge führen, Verfahren zur Kenntnisnahme, Einbindung und Kommunikation berücksichtigter Stakeholder-Interessen) (vgl. a.a.O: 10). Das Monitoring des NMS ist mehrstufig. Es beinhaltet zum einen die regelmäßige „Analyse und Bewertung von Daten“. So sind nachhaltigkeitsrelevante Arbeitsabläufe und Tätigkeiten im Auge zu behalten und zu messen, die Zielerreichung des Verbesserungsprogramms zu überwachen, wie auch die Erfüllung von gesetzlichen Vorschriften, Standards etc. Verfahren zur Reaktion auf Abweichungen müssen implementiert werden. Durch, vom zu auditierenden Bereich unabhängige, Auditor*innen sind mindestens jährlich einmal ,interne Audits“ (Nachhaltigkeitsbetriebsprüfungen) wirkungen dargestellt, das Gesamtsystem transparent abgebildet ist (vgl. kate e.V. 2012: 9). 
durchzuführen. Dies dient der Leitung zur Überwachung, Steuerung und kontinuierlichen Verbesserung des NMS. Im Audit werden Abweichungen festgestellt und Empfehlungen zur Optimierung des NMS festgehalten. Zweitens erfolgt eine „Bewertung des NMS durch die oberste Leitung“. Diese Bewertung erfolgt dokumentiert und enthält ggf. Anpassungsmaßnahmen. Eine unabhängige und neutrale Stelle (zugelassene, von der Deutsche Akkreditierungs- und Zulassungsgesellschaft für Umweltgutachter (DAU) akkreditierte, Gutachter*innen mit gesonderter Qualifizierung durch den Systemgeber von EMASplus) übernimmt das ,externe Audit“, dass eine zweifache Prüfungsfunktion umfasst: eine Systemprüfung (funktioniert das NMS?) und eine Performanceprüfung (gibt es Verbesserungspotenzial bei der Nachhaltigkeitsleistung?). Das externe Audit umfasst eine Dokumentenprüfung und eine Vor-OrtPrüfung (Befragungen, Begehungen und Begutachtung von Nachweisen). Der (jährlich zu aktualisierende) Nachhaltigkeitsbericht umfasst Ausführungen zu den oben beschriebenen diversen Elementen des NMS und ist Grundlage der Zertifizierung der Organisation und ihres NMS bzw. der nachfolgenden Validierung (alle 3 bzw. 4 Jahres) des Berichts. Ziel des Berichtes ist es die Öffentlichkeit über die Nachhaltigkeitsleistung des Unternehmens zu informieren (a.a.O.: 13f.).

Es kann (normativ gewünscht) sein, dass Organisation per se nachhaltig agieren und wirken, ein ,spezifizierter“, ,parallel“ laufender Nachhaltigkeitsmanagementansatz zu einem zukünftigen Zeitpunkt obsolet ist/wird. In diesem Sinne könnte, wie auch bei den unterschiedlichen CSR-Managementansätzen nach der Balanced Scorecard (insbesondere „CSR als 5. Perspektive“ und „CSR-BSC“), auch bei EMASplus die Frage gestellt werden, inwieweit es Sinn macht oder machen kann, ein Nachhaltigkeitsmanagement zwar als integriertes aber doch ,fokussiertes“ Managementsystem aufzusetzen. Auch an dieser Stelle muss darauf hingewiesen werden, dass hier der fokussierte Blick auf die Dinge gewürdigt werden kann/muss (normativ), wie auch der Versuch, damit den Herausforderungen einer unbeständigen, unsicheren, komplex, ambiguitären Welt (,VUCA-Welt") gerecht zu werden, unternehmerische gesellschaftliche Verantwortung fassbar zu machen, mit ,gültigen“ Inhalten zu versehen, dieser zu entsprechen, schlicht diese aktiv zu steuern/zu managen. Für die Unternehmen kann dem damit auch eine ganz praktische Dimension innewohnen. Es kann davon ausgegangen werden, dass eine Vielzahl der profitund sozialwirtschaftlichen Organisationen (noch) nicht derart aufgestellt sind/sich in einer derartigen Perspektive ihrem eigenen Unternehmen ,nähern", dass nachhaltige Strukturen als selbstverständlicher Teil des Unternehmens mitgesteuert werden bzw. diese nachhaltigen Implikationen selbst das Unternehmen lenken (können/sollen) (ganz abgesehen von diversen Rahmenbedingungen, die gesellschaftliche Verantwortung und Nachhaltigkeit nicht als Selbstläufer stützen). So muss auch EMASplus (wie auch andere nachhaltigkeitsspezifische Managementsysteme) als Möglichkeit gesehen werden, den 
Blick des Unternehmens zunächst auf nachhaltiges Handeln zu fokussieren, ggf. zu ändern. Gleichermaßen erlaubt die Organisation damit auch der Gesellschaft den Blick auf das Unternehmen und ermöglicht dieser damit, nachvollziehen zu können, ob und inwiefern eine Organisation gesellschaftlich verantwortlich handelt.

\section{CSR-BSC und EMASplus im Fazit}

Die beiden vorgestellten Managementsysteme zur Verankerung von CSR erlauben es profit- und sozialwirtschaftlichen Unternehmen bzw. Organisationen im Rahmen und auf Basis eines (deutschen) wirtschaftspolitischen Modells „Soziale Marktwirtschaft“ und eines sozialpolitischen Modells des Wohlfahrtsstaates, im Rahmen diverser international ausgerichteter Standards, Guidelines (z.B. CSR-Richtlinie, Sozialstandards der ILO, UN Global Compact) gesellschaftlicher Verantwortung bzw. Nachhaltigkeit Ausdruck zu verleihen bzw. diese zu gestalten. Ob diese Managementsysteme auch als Schritte hin zu aktuell und alternativ diskutierten Wirtschafsmodellen - wie z.B. der Gemeinwohlökonomie ${ }^{19}$ - sind, sein sollen oder können bzw. auch in Rahmen solcher Wirtschaftsmodelle ihren Sinn machen können, soll an dieser Stelle nicht weiter diskutiert werden. Dadurch, dass es sich um umfassende Managementsysteme handelt bieten sie jeweils einen weitreichenden Ansatz, der es erlaubt sich der hohen Komplexität CSR-bedingter unternehmerischer, sozialen bzw. gesellschaftlichen bzw. persönlicher Herausforderungen anzunähern und gangbare Wege zu finden.

Die beiden Ansätze unterscheiden sich stark darin, inwiefern sie bereits vom Grunde her thematisch/inhaltlich auf das Thema CSR rekurrieren. Hier ist offensichtlich EMASplus vom Grund her spezifisch(er). Die BSC ist an sich „CSR-neutral“, muss aber nichtdestotrotz insbesondere beim Ansatz „CSR als fünfte Perspektive“ und bei der „CSR-BSC“ bereits relativ konkret werden, auf welche Nachhaltigkeitsziele Bezug genommen werden soll, spätestens in der Umsetzung z.B. der CSR-BSC muss hier klar definiert und gehandelt werden. An dieser Stelle kann überlegt werden, ob ein Nachhaltigkeitsmanagementsystem wie EMASplus als Umsetzungsinstrument herangezogen wird.

19 Im Sinne einer alternativen Wirtschaftsordnung (liberale und ethische Marktwirtschaft), ,die nicht auf Gewinnstreben und Konkurrenz beruht, sondern auf Gemeinwohl-Streben und Kooperation" (Internationaler Verein zur Förderung der Gemeinwohl-Ökonomie e.V. 2019: 1). Unternehmerische Erfolgsindikatoren sind nicht finanzielle Kennzahlen, sondern Indikatoren, welche den Beitrag des Unternehmens zum Gemeinwohl deutlich machen. 
Im Weiteren kann für beide Ansätze zusammengefasst werden,

- sie können beide stimmig für jedwede Unternehmensgröße ausgebildet werden,

- dass sie sowohl in profit- als auch in sozialwirtschaftlichen Organisationen als Managementsysteme funktionieren können,

- beide stehen nicht im Widerspruch dazu, dass es unterschiedliche, i.d.R. interdependente, Triebfedern (Business/Social/Personal Case) gibt, warum CSR systematisch als Wesenszug im Unternehmen verankert werden soll/möchte, dass insbesondere aber EMASplus durchaus auch (normativ) transportiert, dass gesellschaftliche Verantwortung bzw. Nachhaltigkeit um ihrer selbst willen zu realisieren ist (nicht um der Gewinnmaximierung zu dienen),

- dass sie sich nicht ausschließen: ein BSC-orientiertes Management des Unternehmens kann dazu führen, dass eine EMASplus-Zertifizierung angestrebt wird. Ein Management nach EMASplus kann die grundlegende mehrperspektivische Betrachtungsweise unternehmerischen Geschehens initiieren, welche bereits auf normativer und strategischer Ebene CSR als „Leitkonzept" integriert,

- dass sie Daten für eine entsprechende Berichterstattung liefern können, z.B. nach der CSR-Richtlinie,

- dass sie nur dann zu Erfolg und Wirkung führen, wenn sie jeweils konsequent gesteuert und umgesetzt werden.

\section{Literatur}

Ackermann, K.-F. (2000): Das Balanced Scorecard-Konzept: Grundlagen und Bedeutung für die Unternehmenspraxis, in ders. (Hrsg.): Balanced Scorecard für Personalmanagement und Personalführung: Praxisansätze und Diskussion. Wiesbaden: Gabler: 11-45.

Berens, W./Karlowitsch, M./Mertes, M. (2000): Die Balanced Scorecard als Controllinginstrument in Non-Profit-Organisationen. In: Controlling 2000, 1: 23-28.

BMAS (o.J.): Was ist CSR - Nachhaltigkeit und CSR. https://www.csr-in-deutschland.de/DE/Was-ist-CSR/Grundlagen/Nachhaltigkeit-und-CSR/csr-grundlagen.html [Zugriff: 20.06.2019].

Dansk Standard (Hrsg.) (2011): DS 49001:2011 - Managementsystem für gesellschaftliche Verantwortung - Anforderungsbeschreibung. Berlin: Beuth Verlag.

Dietsche, C./Lautermann, C./Westermann, U. (2019): CSR-Reporting von Großunternehmen und KMU in Deutschland 2018. In: IWÖ/future (Hrsg.): https://www.ranking-nachhaltigkeitsberichte.de/data/ranking/user_upload/2018/Ranking_Nachhaltigkeitsberichte_2018_Ergebnisbericht.pdf [Zugriff: 15.06.2019].

DIN Deutsches Institut für Normung (2011): Leitfaden zur gesellschaftlichen Verantwortung (ISO 26000:2010). Berlin: Beuth Verlag.

Eichmann, T. (2016): Zarte Pflanze Nachhaltigkeit. In: WOHLFAHRTINTERN 2016, 5 S. 30-32. 
EMASplus (о.J.): Zertifizierungsprozess. https://www.emasplus.org/zertifizierungsprozess. [Zugriff: 22.06.2019].

Friedag H. R./Schmidt, W. (2002): Balanced Scorecard: Mehr als ein Kennzahlensystem. Freiburg im Breisgau: Haufe.

Frischen, K./ Lawaldt, A. (2008): Social Entrepreneurship - Theorie und Praxis des Sozialunternehmertums. In: Stiftung \& Sponsoring, 11, 6. S. 2-16.

Geschäftsstelle des Umweltgutachterausschusses (Hrsg.) (2018): EMAS - Mehrwert schaffen, Risiken vermeiden. Die Stärken von EMAS gegenüber der ISO 14001. https:/www.emas.de/fileadmin/user_upload/06_service/PDF-Dateien/Mit-EMASMehrwert-schaffen_Vergleich-ISO14001.pdf [Züuriff: 22.01.2019].

Global Value Toolkit (o.J.): WHAT TO EXPECT. http: https://www.globalvalue.eu/toolkit/ [Zugriff: 15.06.2019|.

Gmür, M. (2000): Strategisches Management für Nonprofit-Organisationen. In: Nährlich:/Zimmer, A. (Hrsg.): Management in Nonprofit-Organisationen. Opladen: Leske + Budrich: 177-200.

Grunwald, K. (2014): Sozialwirtschaft. In: Arnold, U./Grunwald, K./ Maelicke, B. (Hrsg.): Lehrbuch der Sozialwirtschaft. 4. Aufl. Baden-Baden: Nomos. S. 33-63.

Habisch, A./Schwarz, C. (2015): CSR als Investition in Human- und Sozialkapital. In: Schneider, A./Schmidpeter, R. (Hrsg.): Corporate Social Responsibility. Verantwortungsvolle Unternehmensführung in Theorie und Praxis. Berlin, Heidelberg: Springer: 113-133.

Haddad, T. (1998): Balanced Scorecard. In: Eschenbach, R. (Hrsg.): Führungsinstrumente für die Nonprofit-Organisation. Bewährte Verfahren im praktischen Einsatz. Stuttgart: Schäffer-Poeschel: 58-64.

Hoffmann, E./Dietsche, C./Westermann, U. [u.a.] (2016): Nachhaltigkeitsberichterstattung in Deutschland 2015. https://www.ranking-nachhaltigkeitsberichte.de/data/ranking/user_upload/2015/Ranking_Nachhaltigkeitsberichte_2015_Ergebnisbericht.pdf [Zugriff: 15.06.2019].

Hofmacher, P. (2017): Nachhaltigkeitsbericht. Das Bewusstsein schärfen. In: WOHLFAHRTINTERN 2017, 5: 36.

Internationaler Verein zur Förderung der Gemeinwohl-Ökonomie e.V. (2019): Gemeinwohlökonomie. Ein Wirtschaftsmodell mit Zukunft. Erst-Info. https:/www.ecogood.org/media/filer_public/cd/2a/cd2a3681-fd69-4de5-8ba30240766bb98c/erstinfo.pdf [Zugriff: 22.06 .2019 ]

IWÖ/future (2012): IÖW/future-Ranking Nachhaltigkeitsberichte 2011. https://www.ranking-nachhaltigkeitsberichte.de/data/ranking/user_upload/pdf/Ranking_2011_Ergebnisse_KMU.pdf [Zugriff: 15.06.2019].

Kaplan, R. S./Norton, D. P. (1997): Balanced Scorecard. Strategien erfolgreich umsetzen. Stuttgart: Schäffer-Poeschel.

Kaplan, R. S./Norton, D. P. (2001): Die strategiefokussierte Organisation. Führen mit der Balanced Scorecard. Stuttgart: Schäffer-Poeschel.

kate e.V. (o.J.): Systemgeber des EMASplus Systems. Wie ist EMASplus entstanden. https://www.emasplus.org/systemgeber [Zugriff: 11.06.2019].

kate e.V. (2012): Richtlinie Nachhaltigkeitsmanagement EMAS ${ }^{\text {plus }}$. Elemente und Anforderungen eines Nachhaltigkeitsmanagements auf der Grundlage von EMAS und ISO 26000. https://www.emasplus.org/richtlinie-emasplus (Zugriff: 21.05.2019). 
Lang, R./Dresewski, F. (2010): Zur Entwicklung des Social Case zwischen Unternehmen und Nonprofit-Organisationen. In: Backhaus-Maul, H./Biedermann, C./Nährlich:/Polterauer, J. (Hrsg.): Corporate Citizenship in Deutschland. Gesellschaftliches Engagement von Unternehmen. Bilanz und Perspektiven. 2. Aufl. Wiesbaden: VS Verlag für Sozialwissenschaften: 401-422.

Lotter, D./Braun, J. (2016/2017): Der CSR Manager. forum Serie Teil 12 und 13. In: forum Nachhaltig Wirtschaften 2016, 2: 107-109 und 2017, 2: 66-68.

MW (Malteser Werke) (2013): 1. Nachhaltigkeitsbericht der MW Malteser Werke gGmbH, Köln. https://www.malteser-werke-ggmbh.de/fileadmin/Files_sites/Fachbereiche/Werke/Downloads/20130712_Nachhaltigkeitsbericht_2013.pdf [Zugriff: 21.06.2019].

Nowrot, K. (2018): Gesellschaftliche Verantwortung von Unternehmen - Rechtswissenschaftliche Zugänge und Betrachtungsweisen. In: Backhaus-Maul, H./Kunze, M./Nährlich: (Hrsg.): Gesellschaftliche Verantwortung in Deutschland. Wiesbaden: Springer VS: 215-233.

Richtlinie 2014|95|EU des Europäischen Parlaments und des Rates vom 22. Oktober 2014 zur Änderung der Richtlinie 2013|34|EU im Hinblick auf die Angabe nichtfinanzieller und die Diversität betreffender Informationen durch bestimmte Unternehmen und Gruppe (ABl. vom 15.11.2014, L330/1-L330/9).

Riess, B. (2010): Unternehmensengagement - ein Beitrag zur gesellschaftlichen Selbststeuerung zwischen Markt und Staat. In: Backhaus-Maul, H./ Biedermann, C./Nährlich: [u.a.] (Hrsg.): Corporate Citizenship in Deutschland. Gesellschaftliches Engagement von Unternehmen. Bilanz und Perspektiven. 2. Aufl. Wiesbaden: VS Verlag für Sozialwissenschaften: 588-600.

Rusche, T. (2019): Methodische Schärfung der CSR-Diskussion. In: Stoll, B./Herrmann, H. (Hrsg.): Impulse zur Corporate Social Responsibility in Sozial- und Profitwirtschaft. Opladen: Barbara Budrich Verlag: 105-121.

Schaltegger: (2018): Unternehmerische Verantwortungsübernahme für gesellschaftlichen Nutzen. In: Backhaus-Maul, H./Kunze, M./Nährlich: (Hrsg.): Gesellschaftliche Verantwortung in Deutschland. Wiesbaden: Springer VS: 351-364.

Stoll, B. (2004): Soziale Verantwortung in und durch Kleine und Mittlere Unternehmen. Das COSORE-Konzept zur geschäftsintegrierten und nutzenorientierten Umsetzung. In: Stiftung \& Sponsoring, 2004, 1: 32-34.

Stoll, B. (2009): Sozial und ökonomisch handeln. Corporate Social Responsibility kleiner und mittlerer Unternehmen. Frankfurt/New York: Campus Verlag.

Stoll, B. (2013): Balanced Scorecard für Soziale Organisationen: Qualität und Management durch strategische Steuerung. 3. Aufl. Regensburg: Walhalla Fachverlag.

UGA (o.J. a) Corporate Social Responsibility soll messbar werden mit EMAS. https:/www.emas.de/rechtliche-grundlagen/nachhaltigkeit/iso26000/ [Zugriff: 11.06.2019].

UGA (o.J. b): Was ist EMAS? EMAS das Gütesiegel der Europäischen Union. http://www.emas.de/ueber-emas/ [Zugriff: 11.06.2019].

UGA (2006): Stellungnahme des Umweltgutachterausschusses zum Dialog-Entwurf für eine Empfehlung des Rates für Nachhaltige Entwicklung an die Bundesregierung und die Wirtschaft zur Verantwortung von Unternehmen für eine nachhaltige Entwicklung (CSR). https://www.emas.de/fileadmin/user_upload/05_rechtliches/PDF-Dateien/UGA_Stellungnahme_CSR-Entwurf.pdf [Züuriff: 22.06̄.2019]. 
UGA (2012): Die ISO 26000 unter der EMAS Lupe. https://www.emas.de/fileadmin/ user_upload/06_service/PDF-Dateien/ISO_26000_unter_der_EMAS-Lupe.pdf [Zugriff: 11.06.2019]. 


\section{Corporate Social Responsibility als Instrument der Mitarbeitergewinnung: Gründe für die gestiegene Relevanz von CSR und zielgruppenspezifische CSR- Kommunikation mit den Sinus-Milieus ${ }^{\circledR}$}

\section{Christoph Schleer}

Studien zeigen auf, dass Unternehmen, die sich für gesellschaftliche Belange engagieren, einen Wettbewerbsvorteil im ,war for talents“ erzielen. Begründet wird das mit steigenden CSR-Erwartungen der Bewerber*innen. Nur: Wie lässt es sich erklären, dass die Forderungen gegenüber Unternehmen, sich für soziale und ökologische Zwecke einzusetzen, zunehmen? Und: Wie kann es gelingen, die Übernahme von Verantwortung zielgruppengerecht zu kommunizieren?

\section{Gründe für die gestiegenen CSR-Erwartungen}

\subsection{Wachsende Sorge um soziale und ökologische Missstände}

$\mathrm{Zu}$ Beginn des 21. Jahrhunderts stehen die Gesellschaften der Industrie- und Entwicklungsstaaten vor großen ökonomischen, sozialen und ökologischen Herausforderungen: Nach seriösen Schätzungen werden die weltwirtschaftlichen Aktivitäten bis 2050 um das Fünf- bis Sechsfache ansteigen (Sachs 2010: 45). Damit nimmt der Energie- und Rohstoffhunger der Welt deutlich zu. Als Folge wächst der Druck auf die Ökosysteme; Schadstoffe sammeln sich in der Luft, an Land und im Wasser. Dies wiederum beschleunigt den Klimawandel, führt zu Süßwasserknappheit, Entwaldung und abnehmender biologischer Vielfalt (WWF 2008: 4). Hinzu kommt der Anstieg der Weltbevölkerung: Heute leben etwa 7,7 Milliarden Menschen auf der Erde, laut Prognosen sollen es im Jahr 2050 über 9,7 Milliarden sein (United Nations 2019). Dabei wächst die Bevölkerung ausgerechnet in den ärmsten Teilen der Welt. Ohne eine Stabilisierung der Weltbevölkerung werden die illegalen Flüchtlingsströme noch 
zunehmen, globale Auswirkungen auf Frieden und Stabilität nicht ausbleiben (Sachs 2010: 222).

Bei den Bürger*innen bleiben die sich ändernden sozialen und ökologischen Realitäten nicht unbemerkt. Durch den allgemeinen Anstieg des Bildungsniveaus und den zahlreichen Informationsangeboten der Medien, sind sie schneller denn je in der Lage, sich ein Bild über aktuelle und künftige Veränderungen auszumalen. Vor diesem Hintergrund kann es nicht wundern, dass die fast täglich erscheinenden Meldungen über soziale und ökologische Missstände zu einer wachsenden Sorge in der Bevölkerung führen. Viele Bürger*innen tragen diese Sorge auch an Unternehmen heran, denn ohne die Anstrengungen von Unternehmen wird man globale Herausforderungen nicht bewältigen können.

\subsection{Bei der beruflichen Orientierung gewinnen weiche Faktoren an Bedeutung}

Zunehmend sind es weiche Faktoren, die die Arbeitgeberattraktivität bestimmen. In einer repräsentativen Online-Befragung des SINUS-Instituts gaben 85 Prozent der 14- bis 24-jährigen Baden-Württemberger*innen an, den Faktor Spaß an der Arbeit zu ihren Top fünf der wichtigsten Berufskriterien zu zählen (Schleer/ Calmbach 2015). Für 65 Prozent muss der Beruf den eigenen Neigungen und Fähigkeiten entsprechen und 58 Prozent heben die Vereinbarkeit von Beruf und Privatleben hervor. Die Möglichkeit, sich selbst verwirklichen zu können (49 Prozent), wird ähnlich häufig wie das Einkommen (51 Prozent) in die Top fünf der wichtigsten Berufskriterien gezählt.

Auch bei den Erwartungen an Unternehmen steht nicht das Gehalt auf Rang eins der Prioritätenliste; ganz oben stehen ein sehr gutes Verhältnis zwischen Mitarbeitenden und Vorgesetzten sowie eine sehr gute Stimmung unter den Kollegen. 53 respektive 51 Prozent der Befragten ist dies ,äußerst wichtig“. Eine überdurchschnittlich gute Bezahlung ist hingegen für deutlich weniger zentral (,,äußerst wichtig“: 38 Prozent). Und dies gilt nicht nur für junge Baden-Württemberger*innen. Ein Blick auf die Ergebnisse der bundesweit durchgeführten McDonald's Azubistudie zeigt, dass Spaß und Freude an der Arbeit von Jugendlichen und jungen Erwachsenen wesentlich häufiger mit „besonders wichtig“ bewertet wird als das Einkommen (vgl. Abbildung 1).

Dennoch spielt das Gehalt natürlich keine untergeordnete Rolle. Als Hygienefaktor ist und bleibt das Gehalt von grundlegender Bedeutung. Allerdings zeigen die Befunde, dass ein gutes Gehalt alleine nicht (mehr) ausreicht. Vielmehr ist anzunehmen, dass immer mehr Arbeitnehmer*innen - innerhalb selbst abgesteckter Grenzen - zwischen einem höheren Gehalt und einem attraktiveren Arbeitsumfeld abwägen. 
Abbildung 1: Erwartungen an die berufliche Tätigkeit

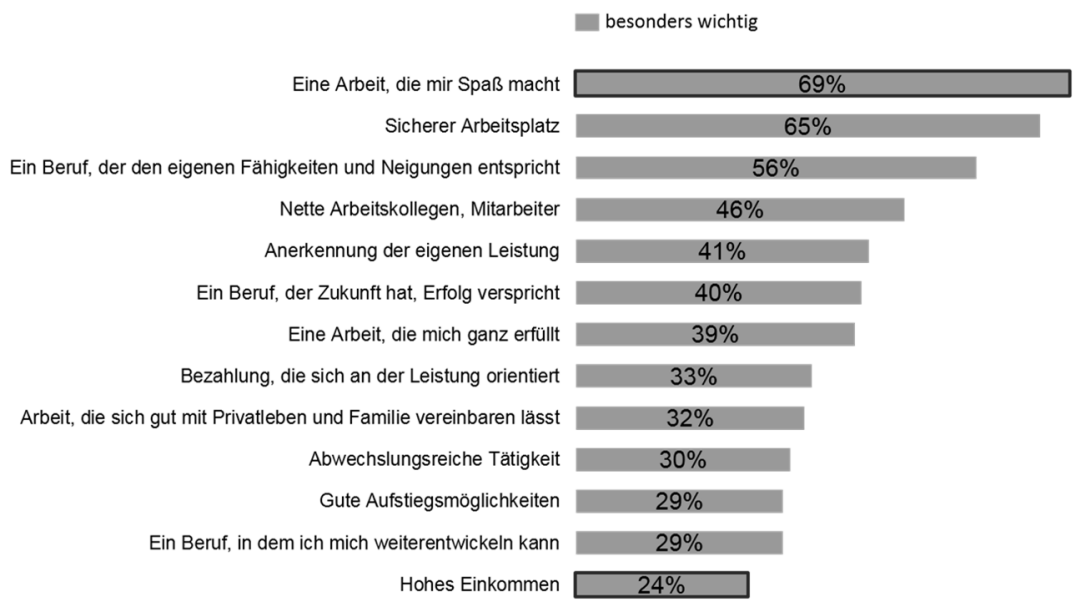

Basis: 15- bis 24-jährige Jugendliche in Deutschland $(N=1.674)$

Quelle: IfD Allensbach, McDonald's Ausbildungsstudie 2015

\subsection{Berufseinsteiger nehmen einen deutlichen Anforderungsdruck wahr}

Dass bei vielen jungen Menschen weniger die materiellen als vielmehr die immateriellen Faktoren die Attraktivität von Beruf und Unternehmen bestimmen, gilt umso mehr, da viele von ihnen einen zunehmenden Leistungsdruck wahrnehmen: In der erwähnten SINUS-Studie gaben 51 Prozent der jungen BadenWürttemberger*innen an, sich Sorgen zu machen, den Anforderungen der heutigen Berufswelt nicht gewachsen zu sein. Jeder Zweite äußerte sogar Angst vor dem Leistungsdruck in der Arbeitswelt. Und auch diese Ergebnisse decken sich mit den Befunden der McDonald's Azubistudie. Danach sind 68 Prozent der befragten 15- bis 24-Jährigen davon überzeugt, dass junge Menschen heute unter höherem Leistungsdruck stehen als früher.

\subsection{Potenzielle Mitarbeitende schließen von dem Engagement eines Unternehmens auf das Betriebsklima und die vorherrschenden Arbeitsbedingungen}

Vor diesem Hintergrund wundert es nicht, dass immer mehr Unternehmen CSR als Instrument im Nachwuchs- und Personalmarketing bemühen. Denn wie einschlägige Forschungsergebnisse zeigen, schließen Bewerber*innen von 
der moralisch-ethischen Ausrichtung eines Unternehmens auf das Betriebsklima und die vorherrschenden Arbeitsbedingungen (Greening/ Turban 2000). Mit anderen Worten gehen potenzielle Bewerber*innen davon aus, dass in Unternehmen, die sich für soziale, ökologische und andere ethische Belange einsetzen, auch der Umgang mit den Mitarbeitenden dementsprechend ausfällt. Vor allem dann, wenn junge Talente die Wahl haben, legen sie immer größeren Wert darauf, in Unternehmen zu arbeiten, die Verantwortung in der Gesellschaft übernehmen (Albinger/ Freeman 2000). Dabei äußern viele Mitarbeitende auch den Wunsch, sich selbst aktiv engagieren zu können. So geben in der aktuellen Markt-Media-Studie „Best4Planning“ 44 Prozent der bundesweit befragten Bürger*innen ( $\mathrm{N}=30.121)$ an, ideal sei ein Beruf, in dem man ökologisches und soziales Engagement verwirklichen kann (gik 2017).

\section{CSR-Leistungen zielgruppenspezifisch kommunizieren}

Damit CSR seine positive Wirkung entfalten kann, benötigen potenzielle Mitarbeitende ein Mindestmaß an Information, Wissen und Urteilsvermögen. Wie Studien zeigen, können aber nur wenige Bürger*innen Unternehmen nennen, die ihnen durch ein besonderes soziales oder ökologisches Engagement aufgefallen sind (Auger et al. 2003; Belk/ Devinney/ Eckhardt 2005; Boulstridge/ Carrigan 2000; Carrigan/ Attalla 2001; Sen/ Bhattacharya/ Korschun 2006). Und auch das Wissen um unethische Marketing- und Geschäftspraktiken beschränkt sich oft auf vage Kenntnisse über Meldungen aus der Medienlandschaft (Boulstridge/ Carrigan 2000; Carrigan/ Attalla 2001). Verantwortlich handelnde Unternehmen, die die CSR-Erwartungen der Bewerber*innen ernst nehmen, müssen also verstärkt in Informationsaktivitäten investieren. Damit stehen sie nicht nur vor der Herausforderung, ihre sozialökologischen Leistungen sichtbar und glaubwürdig zu kommunizieren (Schleer 2014), sondern die Kommunikation auch zielgruppenspezifisch anzugehen. Denn: CSR-Leistungen werden unterschiedlich wahrgenommen und bewertet. Tatsächlich verfügen potenzielle Mitarbeitende über unterschiedliche Zugänge zum Themenkomplex CSR. Inwiefern Unternehmen in den Augen der Bewerber*innen verantwortlich agieren, ist nicht von einer umfassenden Bewertung aller denkbaren CSR-Aspekte abhängig, sondern wird vielmehr an subjektiv relevanten Einzelaspekten festgemacht. Folglich muss CSR-Kommunikation inhaltlich, sprachlich und ästhetisch auf die anvisierten Zielgruppen ausgerichtet sein. Und da es immer schwieriger wird, die wenigen geeigneten Bewerber*innen $\mathrm{zu}$ finden, ist es umso wichtiger, dass diese gezielt angesprochen werden. Damit das Nachwuchs- und Personalmarketing bei den richtigen Personen ankommt, ist ein klarer Fokus notwendig. Ganz deutlich wird dies bei Führungs- 
kräften, die meist nur noch über spezialisierte Dienstleistende rekrutiert werden können. Bei der aktuellen Marktlage können aber auch Berufseinsteigende Erwartungen formulieren, zwischen Arbeitgeber*innen vergleichen und ihre Entscheidungen abwägen.

\section{CSR-Kommunikation mit den Sinus-Milieus ${ }^{\circledR}$}

Der gesellschaftswissenschaftliche Ansatz von SINUS unterstützt Unternehmen, CSR-Aktivitäten zielgruppengerecht zu kommunizieren, weil er dort ansetzt, wo sich Einstellungs- und Verhaltensweisen grundlegend erklären lassen - an den Wertorientierungen und Lebensstilen sozialer Milieus.

Zur Entwicklung einer erfolgversprechenden CSR-Kommunikation verwendet das SINUS-Institut den gesellschaftswissenschaftlichen Ansatz der Milieu-Segmentierung. Die Sinus-Milieus ${ }^{\circledR}$ sind ein wissenschaftlich fundiertes Gesellschaftsmodell, das Menschen, die sich in ihrer Lebensauffassung und Lebensweise ähneln, in Gruppen „Gleichgesinnter“ zusammenfasst (vgl. Abbildung 2). Dabei gehen grundlegende Wertorientierungen ebenso in die Analyse ein wie Alltagseinstellungen, Lebensstil und Lebensziele. Dieses umfassende Verständnis ist wichtig, da die Unterschiedlichkeit von Lebensstilen für die Alltagswirklichkeit von Menschen vielfach bedeutsamer ist als die Unterschiedlichkeit sozioökonomischer Lebensbedingungen: Um potenzielle Bewerber*innen mit CSR-Themen zu erreichen, muss man ihre Befindlichkeiten und Orientierungen, ihre Werte, Erwartungen und ihre unterschiedlichen $\mathrm{Zu}$ gänge zu CSR-Themen kennenlernen. Nur dann bekommt man ein wirklichkeitsgetreues Bild davon, was sie bewegt und wie sie bewegt werden können.

Beispielsweise zeigt eine aktuelle SINUS-Studie, dass nur jeder vierte Deutsche glaubt, VW, Audi, BMW und Co. würden strenge Umweltrichtlinien (bei der Produktion und der Beschaffung) verantwortungsbewusst umsetzen. Deutlich macht die Studie aber auch, dass die Befunde stark differenzieren: Je nachdem, welche Sinus-Milieus ${ }^{\circledR}$ in den Bick genommen werden, fallen die Ergebnisse unterschiedlich aus. So ist die Bereitschaft, bei Einhaltung strenger Umweltrichtlinien einen höheren Preis beim Kauf eines PKW zu bezahlen, im Liberal-intellektuellen, Expeditiven und Sozialökologischen Milieu deutlich höher als etwa im Konservativ-etablierten, im Traditionellen oder Prekären Milieu (vgl. Abbildung 3). 
Abbildung 2: Die Sinus-Milieus ${ }^{\circledR}$ in Deutschland 2018

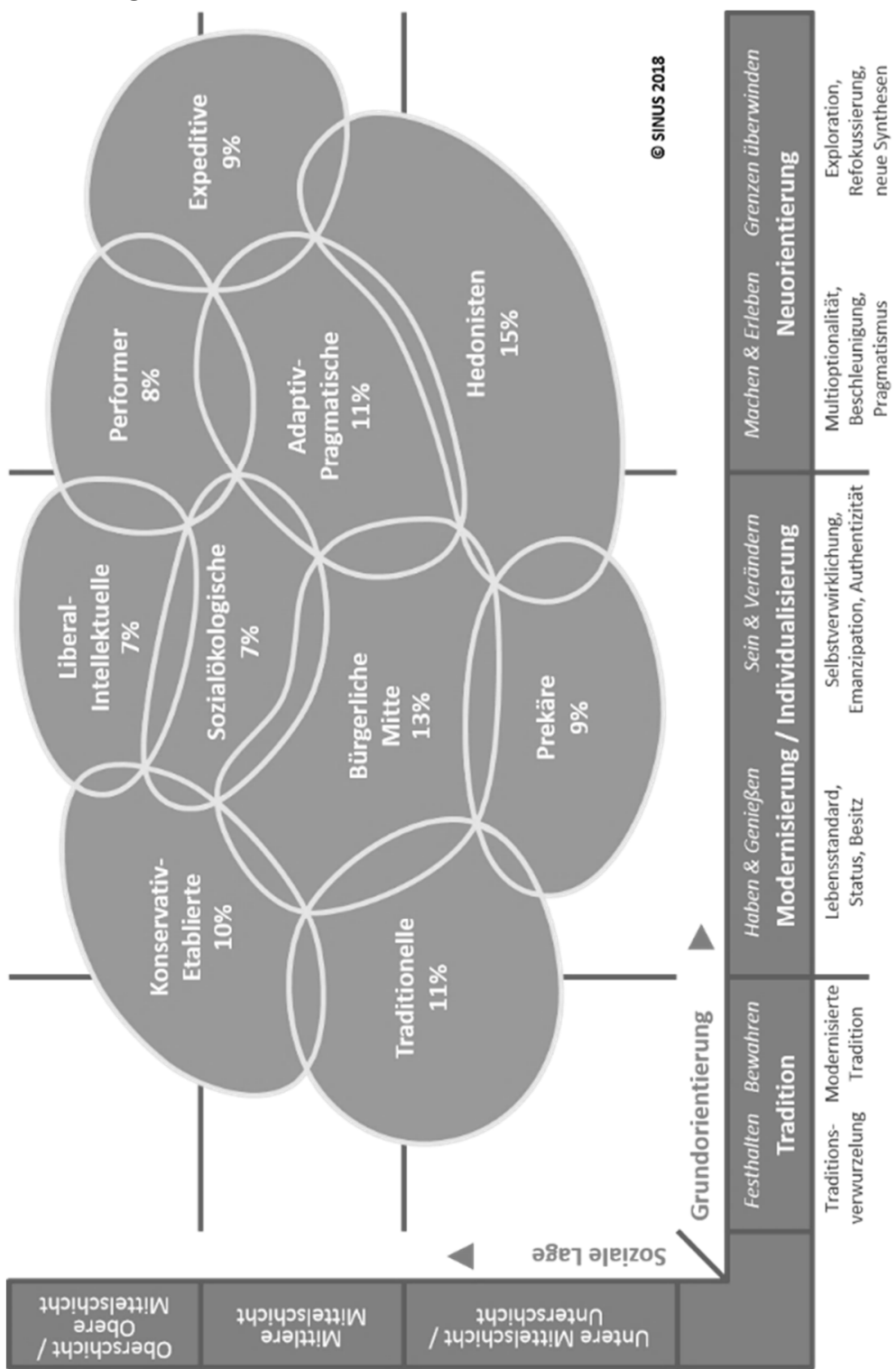

Quelle: SINUS-institut.de 
Abbildung 3: Akzeptanz höherer Preise bei Einhaltung strenger Umweltrichtlinien des Herstellers
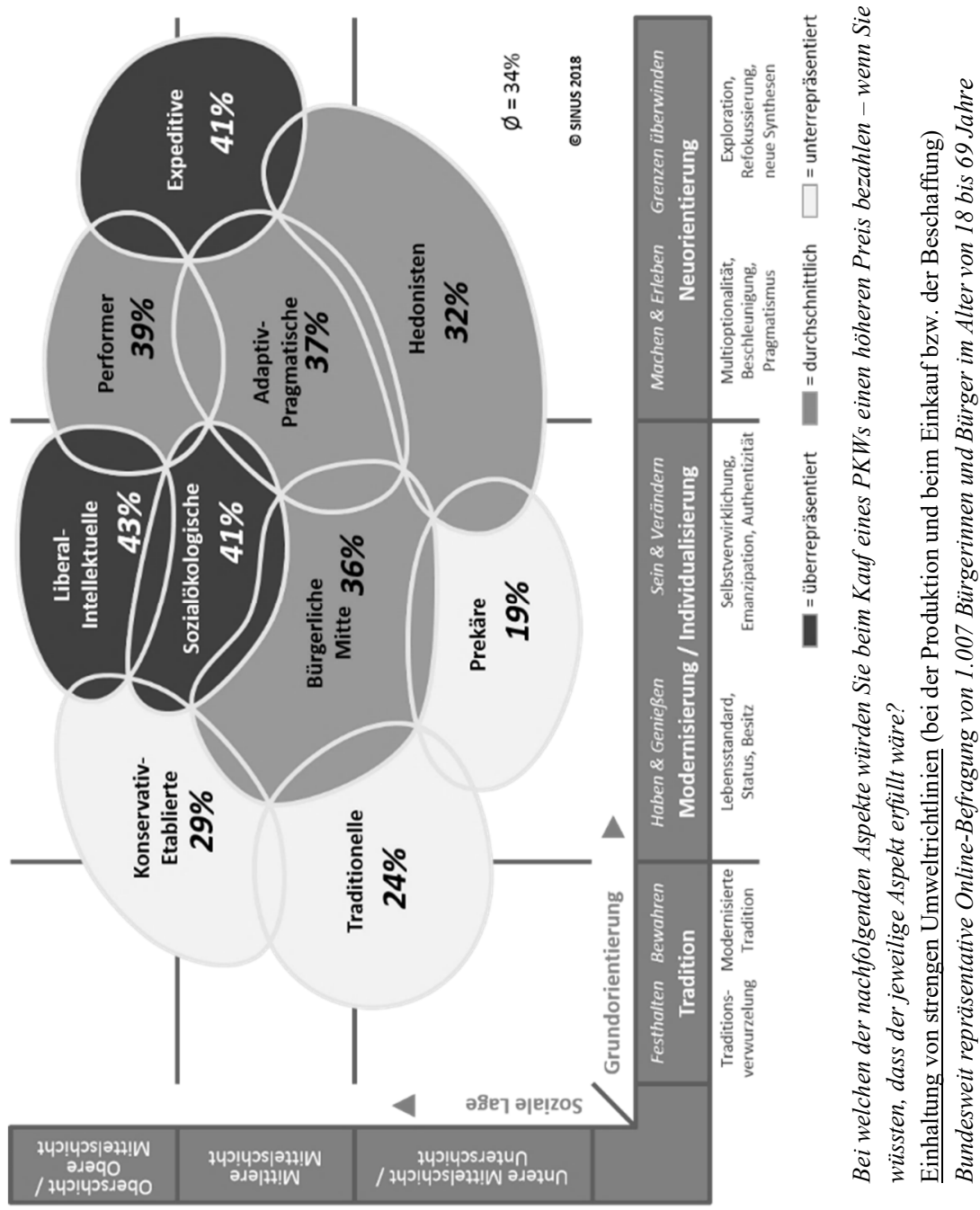

Quelle: SINUS-institut.de 
Dieses Beispiel zeigt, dass die Relevanz von CSR-Themen von den Verbraucher*innen unterschiedlich bewertet wird. Während das Thema Naturschutz etwa im Liberal-intellektuellen Milieu einen hohen Stellenwert einnimmt, stößt es im Konservativ-etablierten Milieu nur auf unterdurchschnittliches Interesse. Zwingend zu berücksichtigen ist aber auch, dass die inhaltlichen $\mathrm{Zu}-$ gänge zum Thema Natur- und Umweltschutz je nach Milieu grundverschieden sein können (vgl. Abbildung 4): Das Kaufkriterium „Einhaltung von strengen Umweltrichtlinien“" (in der Produktion und bei der Beschaffung) kann nur dann Aufmerksamkeit wecken, wenn die für die Zielgruppe passende Argumentation bereitgestellt wird (z.B. Natur als Kulturgut, Lebensqualität durch Umweltschutz, Fortschrittslogik). Erfolgreiche CSR-Kommunikation muss also bedarfs- wie interessengerecht (d.h. unterschiedliche Themenschwerpunkte, unterschiedliche Informationstiefen) und mit Blick auf die unterschiedlichen CSR-Zugänge ausgestaltet sein.

Abbildung 4: Unterschiedliche Zugänge zum Thema Natur- und Umweltschutz

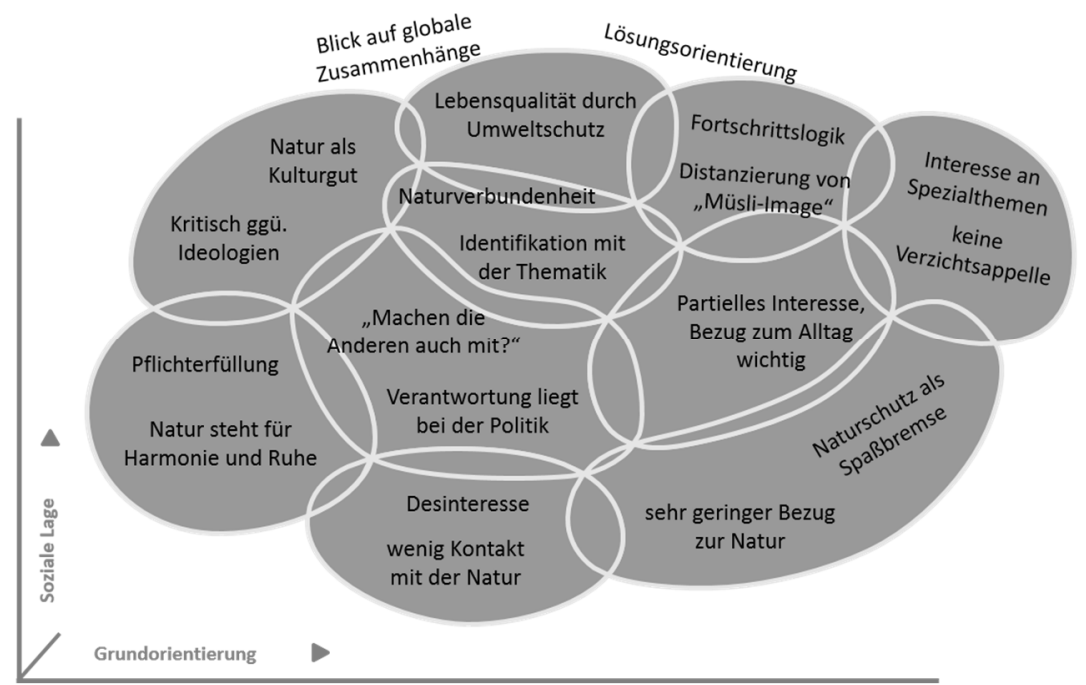

Quelle: SINUS-institut.de 


\section{Literatur}

Albinger, H.S./Freeman, S.J. (2000): Corporate Social Performance and attractiveness as an employer to different job seeking populations. In: Journal of Business Ethics 28, S. 243-253.

Auger, P./Burke, P./Devinney, T.M./Louviere, J.J. (2003): What Will Consumers Pay for Social Product Features? In: Journal of Business Ethics Band 42, 3, S. 281-304.

Belk, R./Devinney, T./Eckhardt, G. (2005): Consumer Ethics across Cultures. Working Paper Series. Nr. 3. UC Berkeley: Center for Responsible Business.

Boulstridge, E./Carrigan, M. (2000): Do consumers really care about corporate responsibility? Highlighting the attitude-behaviour gap. In: Journal of Communication Management 4, 4, S. 355-368.

Carrigan, M./Attalla, A. (2001): The myth of the ethical consumer - do ethics matter in purchase behaviour? In: Journal of Consumer Marketing 18, 7, S. 560-577.

Greening, D.W./Turban D.W. (2000): Corporate Social Performance as a competitive advantage in attracting a quality workforce. In: Business \& Society 39, 3, S. 254289.

gik: Gesellschaft für integrierte Kommunikationsforschung (2017): Best4Planning 2017. Daten sinnvoll verbinden. Springer.

IfD: Institut für Demoskopie. Allensbach (2015): McDonalds Azubistudie: Entschlossen, unentschlossen. Azubis im Land der (zu vielen) Möglichkeiten. Eine Repräsentativbefragung junger Menschen im Alter von 15 bis unter 25 Jahren. München.

Sachs, J.D. (2010): Wohlstand für viele. Globale Wirtschaftspolitik in Zeiten der ökologischen und sozialen Krise. München: Verlag.

Schleer, C. (2014): Corporate Social Responsibility und die Kaufentscheidung der Konsumenten. Wiesbaden: Springer Gabler.

Sen, S./Bhattacharya, C.B./Korschun, D. (2006): The Role of Corporate Social Responsibility in Strengthening Multiple Stakeholder Relationships: A Field Experiment. In: Journal of the Academy of Marketing Science 34, 2, S. 158-166.

Schleer, C./Calmbach, M. (2015): Berufliche Orientierung Jugendlicher. Empirische Hinweise für ein zielgruppenorientiertes Ausbildungsmarketing. In: Barz, H./Jung, M. (Hrsg.): Ausländische Fachkräfte gesucht. Voreilig? Notwendig? Willkommen? Düsseldorf: düsseldorf university press. S. 219-244.

United Nations (2019): https://population.un.org/wpp/Publications/Files/WPP2019 10KeyFindings.pdf [Zugriff: XX)

WWF (2008): Living Planet Report 2008. Gland. Schweiz. 



\title{
Entstehungsfaktoren von Collective-Action-Initiativen zur Lösung sozialer und ökologischer Probleme
}

\author{
Maria Riegler, Markus Scholz
}

\section{Einleitung}

Unter dem Stichwort Corporate Social Responsibility übernehmen Unternehmen zunehmend Verantwortung für gesellschaftliche und ökologische Themen (vgl. Aguinis/ Glavas 2012; Haufler 2001; Vogel 2008). Gleichzeitig entstehen vor dem Hintergrund der Globalisierung, stetig schwächer werdender Nationalregierungen und dem Aufkommen und der Verbreitung disruptiver Technologien gesellschaftliche und ökologische Herausforderungen, die selbst engagierte Unternehmen im Alleingang nicht bewältigen können.

Die global vernetzte Wirtschaft bringt komplexe Zusammenhänge und Abhängigkeiten mit sich, die sowohl Unternehmen als auch Gesetzgeber herausfordern. Diese systemischen Risiken gehen meist über nationalstaatliche Grenzen hinaus und können durch „Dominoeffekte“ ganze Branchen - und in weiterer Folge auch die gesamte Gesellschaft - schädigen (vgl. De Bandt/ Hartmann 2000; Donaldson/ Schoemaker 2013). Systemische Risiken bleiben häufig zu lange unbemerkt, da ihr Ausmaß auf Ebene einzelner Unternehmen meist nicht sichtbar ist: Verhalten, das auf Unternehmensebene als rational erscheint, kann auf Branchenebene verheerende nicht-intendierte Folgen nach sich ziehen (vgl. Donaldson/ Schoemaker 2013). Dies zeigte sich beispielsweise in den weitreichenden Folgen der globalen Finanz- und Wirtschaftskrise 2008, die sowohl Unternehmen als auch die Gesellschaft spürbar belastete.

Insbesondere multinationale Konzerne sind vielfach in unregulierten Bereichen tätig, da einzelne Nationen teilweise nicht fähig oder nicht willens sind, klare gesetzliche Rahmenbedingungen zu schaffen (vgl. Baumann-Pauly et al. 2017; Pattberg 2005). Disruptive Innovation - beispielsweise in der Form rasanten technologischen Fortschritts in der Nano- oder Gentechnologie oder in der Form neuer und disruptiver Geschäftsmodelle wie bei Uber, AirBnB und ähnlichen Diensten - fordert den Gesetzgeber heraus, da es schwierig ist, sie vorausschauend zu regulieren (vgl. Cortez 2014; Guttentag 2015). 
Hinzu kommen ökologische Herausforderungen mit weitreichenden gesellschaftlichen und wirtschaftlichen Folgen wie der Klimawandel und die Knappheit verschiedener Ressourcen (vgl. Global Footprint Network 2018; IPCC 2018; Steffen et al. 2015). Weitere Herausforderungen sind unter anderem globale Migrationsbewegungen, alternde Gesellschaften insbesondere in einigen industrialisierten Ländern und ein abnehmendes Vertrauen der Bürger*innen mancher Länder in etablierte Institutionen (vgl. Cooper 2018; George et al. 2016; Hanitzsch et al. 2018).

Viele der erwähnten Themen sind von existentieller gesellschaftlicher und gleichzeitig von betriebswirtschaftlicher Bedeutung. In diesem Beitrag konzentrieren wir uns auf einen möglichen Ansatz dafür, wie Unternehmen proaktiv mit diesen Themen umgehen und auf diese Weise einen Teil der Lösung bilden können: Collective-Action-Initiativen, die anteilig oder vollständig von privaten Akteuren vorangetrieben werden, um bestimmte Probleme von gesellschaftlicher und betriebswirtschaftlicher Relevanz zu lösen.

\section{Die Insuffizienz staatlicher Interventionen}

Das kontinuierliche Voranschreiten der oben skizzierten gesellschaftlichen und ökologischen Probleme macht deutlich, dass es staatlichen Akteuren bisher nicht gelungen ist, die beschriebenen Themen wirkungsvoll zu behandeln. Dafür gibt es verschiedene Erklärungen. Eine Erklärung in Bezug auf unternehmensrelevante Problemfelder besteht darin, dass staatliche Akteure über zu wenig Insiderwissen verfügen, um überhaupt rechtzeitig systemische Risiken in verschiedenen Branchen erkennen und deren Ausmaß abschätzen zu können (vgl. Donaldson/ Schoemaker 2013). Hinzu kommt, dass dem Gesetzgeber teilweise das Fachwissen und die Sachkompetenz fehlen, um komplexe Bereiche wirkungsvoll zu regulieren (vgl. Auld et al. 2009). Bei disruptiven Innovationen liegt das Regulierungsproblem in der Natur der Sache: In vielen Bereichen sind Gesetzgebungsprozesse langsamer als die wirtschaftlichen und technologischen Entwicklungen, weil Gesetze häufig ex post, also nach dem Auftreten einer disruptiven Innovation, verabschiedet werden (vgl. OECD 2017). Zusätzlich fehlt es in manchen Bereichen schlicht an politischem Willen - beispielsweise, weil einzelne Staaten bei einem regulatorischen Alleingang negative volkswirtschaftliche Konsequenzen zu tragen hätten. Das Resultat können entweder Regulierungslücken oder auch wirkungslose bzw. nicht treffsichere Gesetze sein (vgl. de los Reyes et al. 2017). Donaldson und Schoemaker (2013: 27) beschreiben die Insuffizienz staatlicher Regulierungen wie folgt: „Full reliance on ex-post legislation is like closing the barn door once the horse has fled". 
Zusammengefasst existieren in einigen gesellschaftlich relevanten Bereichen - insbesondere auf transnationaler Ebene - Governance-Lücken und es ist diesbezüglich in naher Zukunft keine substantielle Änderung absehbar. Eine Vernachlässigung dieser relevanten Governance-Lücken hätte spürbare Folgen für die Gesellschaft, aber auch für Unternehmen (vgl. Donaldson/ Schoemaker 2013; Kolk/ van Tulder 2005; Labatt/ Maclaren 1998).

\section{Die Insuffizienz von Einzelinitiativen}

Eine mögliche Antwort auf bestehende gesellschaftliche und ökologische Probleme, die von staatlicher Seite nicht zufriedenstellend gelöst werden, sind CSR-Initiativen einzelner Unternehmen. Diese haben den Vorteil, dass der Koordinationsaufwand relativ gering ist, dass die Initiator*in kaum Kompromissbereitschaft zeigen muss und dass diese Initiativen flexibel, schnell und ohne substantielles Risiko gestartet und verändert werden können. Beispiele für individuelle Initiativen sind firmeneigene Codes of Conduct, andere Regelwerke, die über gesetzliche Regulierungen hinausgehen oder CSR-Projekte, die von einzelnen Organisationen vorangetrieben werden.

So wichtig diese Initiativen sind, haben sie große Schwächen: Erstens ist ein einzelner Akteur häufig gar nicht dazu in der Lage, das Problem, das er lösen möchte, in seiner Gesamtheit zu begreifen und Risiken auf Branchenebene korrekt einzuschätzen (vgl. Donaldson/ Schoemaker 2013). Zweitens ist die Wirkung von Einzelinitiativen meist viel zu klein, um das zugrundeliegende Problem spürbar zu beeinflussen (vgl. Donaldson/ Schoemaker 2013). Drittens erleiden die Akteure, die im Alleingang mit gutem Beispiel vorangehen, mitunter einen Wettbewerbsnachteil gegenüber ihren Mitbewerber*innen. Dieser Wettbewerbsnachteil entsteht, wenn es zu keinen Nachahmereffekten in der Branche kommt, wenn also nur eine Organisation sich selbst dazu verpflichtet, sozialer, ökologischer oder ethischer zu agieren, während die Mitbewerber*innen so fortfahren wie bisher (vgl. Donaldson/ Schoemaker 2013; Huxham/ Macdonald 1992; Vogel 2008). Viertens begegnet die Öffentlichkeit Unternehmen, die eine Initiative mit sozialem, ökologischem oder ethischem Fokus im Alleingang vorantreiben, häufig mit Skepsis dahingehend, ob es sich um ernstgemeintes Engagement oder eine Marketingtaktik handelt (vgl. Jahdi/ Acikdilli 2009; Lock/ Seele 2016). 


\section{Collective Action als möglicher Lösungsansatz}

In einigen Situationen ist daher Collective Action ${ }^{1}$ erfolgversprechender als das individuelle Engagement von Unternehmen. Das gemeinsame Vorgehen von Unternehmen macht es möglich, neue, faire Spielregeln zu definieren, die für alle Akteure gleichermaßen gelten; in der englischsprachigen Literatur wird hierfür der Begriff ,level playing field“ verwendet (Kobrin 2009; Vogel 2010). In Bezug auf Unternehmen bedeutet das Konzept des „level playing field“, dass alle Mitbewerber*innen unter den gleichen Bedingungen agieren können. Gesellschaftliches Engagement verursacht meist Kosten für Unternehmen. Besteht kein „level playing field“, erleiden Unternehmen, die sich für die Lösung sozialer und ökologischer Probleme einsetzen, demnach einen Kosten- und damit Wettbewerbsnachteil. In einer Collective-Action-Initiative können die beteiligten Akteure die neuen Spielregeln so gestalten, dass die betreffenden gesellschaftlichen und betriebswirtschaftlichen Probleme besser reguliert werden und gleichzeitig keiner der Beteiligten einen Wettbewerbsnachteil erleidet (vgl. Pies et al. 2009; Vogel 2010).

Olson (2002/1965) definierte Collective Action als Handlungen, die gesetzt werden, um dem gemeinsamen Interesse einer Gruppe von Individuen zu dienen. Collective-Action-Initiativen im Sinne dieses Beitrags sind Formen der Zusammenarbeit zwischen einem Unternehmen und seinen Stakeholdern mit dem Ziel, Probleme von gesellschaftlicher Relevanz gemeinsam zu lösen (vgl. Brammer et al. 2012; Cashore 2002; Stringham 2015). Unternehmen können hierbei sowohl Verursacher*innen als auch Leidtragende der betreffenden Probleme sein.

Wir unterscheiden nachfolgend zwei unterschiedliche Formen kollektiver Initiativen: Regulierungsinitiativen und Umsetzungsinitiativen. Beide Formen betreffen Initiativen, die von privaten Akteuren vorangetrieben werden und die entweder gänzlich unabhängig von staatlichen Akteuren oder mit staatlicher Unterstützung agieren.

In der ersten Kategorie, jener der Regulierungsinitiativen, sind Initiativen zusammengefasst, in denen private Akteure Normen und Standards betreffend soziale, ökologische oder ethische Fragestellungen schaffen, die über gesetzliche Regelungen hinausgehen. Bekannte Beispiele hierfür sind branchenweite Selbstregulierungssysteme wie das „Responsible Care“-Programm der chemischen Industrie (vgl. King/ Lenox 2000), marktbasierte Governancesysteme wie der Forest Stewardship Council (vgl. Cashore et al. 2007; Turcotte et al. 2014) oder auch die Initiativen innerhalb der Textilindustrie, die sich nach dem

Synonyme oder eng verwandte Begriffe für die im Beitrag beschriebene Art kollektiver Initiativen sind beispielsweise: Multi-Stakeholder-Initiativen, Selbstregulierung, Co-Regulierung, Private Governance, Non-State Market-Driven Governance, New Governance. 
Einsturz einer Textilfabrik in Bangladesch im Jahr 2013 gründeten: Bangladesh Accord on Fire and Building Safety sowie Alliance for Bangladesh Worker Safety (vgl. Donaghey/ Reinecke 2018; Reinecke/ Donaghey 2015).

Die zweite Kategorie, jene der Umsetzungsinitiativen, umfasst solche Initiativen, in denen private Akteure keine Verhaltensregeln entwickeln, sondern konkrete Projekte mit einem sozialen oder ökologischen Nutzen gemeinsam umsetzen. Beispiele hierfür sind regional fokussierte Kooperationsprojekte zur Ausbildung von Fachkräften oder insbesondere auch die „Verantwortungspartner-Regionen“ in Deutschland (vgl. Verantwortungs-partner.de 2014).

Einer der wesentlichen Vorteile von Collective-Action-Initiativen wurde bereits erwähnt: Die Möglichkeit, gravierende gesellschaftliche und betriebswirtschaftliche Probleme zu lösen und dabei gleichzeitig für einen fairen Wettbewerb zu sorgen. Wenn die wichtigsten Marktteilnehmer an einer Initiative beteiligt sind, können die Spielregeln für alle Akteure gleichermaßen geändert werden, wodurch niemand einen Wettbewerbsnachteil aus seinem sozialen, ethischen oder ökologischen Engagement heraus erleiden muss (vgl. Dashwood 2014; Vogel 2010).

Zur Identifikation und Bewältigung systemischer Risiken eignen sich kollektive Initiativen eher als individuelle Ansätze: Erstens ist eine Allianz aus mehreren Organisationen eher in der Lage, vollständige und realistische Risikoszenarien zu entwerfen als das Risikomanagement eines einzelnen Unternehmens und zweitens können in einem kollektiven Prozess effektivere Risikobewältigungsstrategien erarbeitet werden (vgl. Donaldson/ Schoemaker 2013).

Zudem sind die potentiell erzielte Wirkung, die Glaubwürdigkeit und die Legitimität kollektiver Initiativen im Vergleich zu individuellen Initiativen höher - in welchem Ausmaß, das hängt von der konkreten Ausgestaltung der Governance-, Monitoring- und Sanktionsinstrumente der jeweiligen Initiativen ab (vgl. Bernstein/ Cashore 2007; Mena/ Palazzo, 2012; Vogel 2010).

Auch Collective-Action-Initiativen haben Schwächen: Ihre Legitimität wird teilweise in Frage gestellt (vgl. Bäckstrand 2012; Bernstein/ Cashore, 2007; Mena/ Palazzo 2012) und ihre tatsächlich erzielte Wirkung ist manchmal unklar (vgl. King/ Lenox 2000; Vogel 2010). Zur Beseitigung der Legitimitätsprobleme ist es daher wichtig, auf möglichst weitreichende Stakeholderinklusion zu achten (vgl. Mena/ Palazzo 2012). Was die Wirkung der Initiativen betrifft, ist der wichtigste Schritt, nicht nur Absichtserklärungen zu erarbeiten, sondern auch effektive Monitoring- und Sanktionsmechanismen zu etablieren (vgl. Dashwood 2014; Manning/ Reinecke 2016; Vogel 2010). Diese Aspekte sind zwar relevant und werden in der bestehenden Literatur auch ausführlich diskutiert, dieser Beitrag fokussiert jedoch auf eine andere Frage: Aus welchen Gründen beteiligen sich private Akteure an kollektiven Initiativen? 


\section{Unternehmensethische Gründe für „Collective Action“}

Die unternehmensethische Literatur liefert diverse Begründungen dafür, warum Unternehmen sich in kollektiven Initiativen engagieren sollten:

Proponent*innen des Corporate-Citizen-Ansatzes argumentieren, dass in der heutigen globalisierten Welt nicht mehr der Nationalstaat alleine für die Schaffung und Umsetzung von Regeln und die Wahrung von Bürgerrechten zuständig ist (vgl. Matten/ Crane 2005; Scherer et al. 2006). Auch Unternehmen und Nichtregierungsorganisationen nehmen zunehmend regulatorische Aufgaben wahr, die früher staatlichen Akteuren vorbehalten waren (vgl. Scherer et al. 2006). Insbesondere multinationale Konzerne sind bereits vielfältig politisch aktiv: im Schutz sozialer Bedürfnisse, in der Wahrung von Bürgerrechten (beispielsweise durch die Sicherstellung von Meinungsfreiheit unter Mitarbeiter*innen) oder in der Ausübung politischer Rechte (vgl. Matten/ Crane 2005). Basierend auf dieser Beobachtung argumentieren Vertreter*innen des Corporate-Citizen-Ansatzes, dass Unternehmen auch als politische Akteure gesehen werden müssen, die politische Rechte wahrnehmen und gleichzeitig politische Pflichten tragen, welche beispielsweise in der Vermeidung von systemischen Risiken und im Engagement für wichtige ökologische und gesellschaftliche Themen liegen.

Vertreter*innen der normativen Variante des Corporate-Citizen-Ansatzes folgen meist den Argumenten der republikanischen Wirtschaftsethik und der politischen Philosophie Jürgen Habermas' (vgl. Scherer et al. 2006; Steinmann/ Löhr 1996; Steinmann/ Scherer 1998/ 2000; Ulrich 2000/ 2002). Im republikanischen Modell trägt der Bürger eine Doppelfunktion als privater Bürger (Bourgeois) und als Bürger eines Staates oder einer Gemeinschaft, also als Träger politischer Rechte und Pflichten (Citoyen). Diese Doppelfunktion kommt in der republikanischen Wirtschaftsethik auch Unternehmen zu. Diese verfolgen einerseits als private Bürger*innen ihre eigenen Interessen und haben andererseits als Citoyens die Aufgabe, gemeinsam mit den anderen Citoyens Regeln im öffentlichen Interesse zu definieren, die zu einer stabilen und friedlichen Gesellschaft beitragen (vgl. Scherer et al. 2006; Scherer et al. 2014; Scherer et al. 2016). Das öffentliche Interesse ist hierbei nicht als die Summe der Einzelinteressen definiert, sondern als das Ergebnis eines Kommunikationsprozesses, in dem Individuen ihre Präferenzen bilden und verändern. Darüber, dass Unternehmen als politische Akteure tätig sind, besteht unter den genannten Autor*innen kein Zweifel. Um das politische Handeln von Unternehmen auch mit der notwendigen demokratischen Legitimität zu untermauern, schlagen Vertreter*innen des normativen Corporate-Citizenship-Ansatzes vor, das Konzept der deliberativen Demokratie von Jürgen Habermas zu nutzen (vgl. Habermas 1996; Habermas/ Pensky 2001): Um die Legitimität ihres po- 
litischen Handelns sicherzustellen, sollen Unternehmen demnach ihre Aktivitäten demokratisieren - durch kontinuierliche Teilhabe am öffentlichen Diskurs und verstärkte Bemühungen für Transparenz in ihren Aktivitäten (vgl. Scherer et al. 2006). Dazu zählt auch die deliberative Aushandlung privater Standards und Verhaltensregeln unter Einbeziehung der anderen gesellschaftlichen Akteure.

Basierend auf der Annahme universeller und grundlegender Menschenrechte haben einige Autor*innen eine weitere Argumentationslinie für das Engagement von Unternehmen in Collective-Action-Initiativen entwickelt (vgl. de George 2005; Kobrin 2009). Dieser Ansatz fußt auf menschenrechtsbasierten und pflichtethischen Argumenten. Laut den Befürworter*innen des menschenrechtsbasierten Ansatzes entstehen gelegentlich Spannungsverhältnisse zwischen unternehmerischen Tätigkeiten und der Wahrung von Menschenrechten. Immer wieder kommt es zu Fällen von Marktversagen, in denen durch freie ökonomische Austauschhandlungen keine effiziente Verteilung von Gütern und Dienstleistungen gelingt (vgl. King et al. 2012). Informationsasymmetrien sind eine Form von Marktversagen. Diese bestehen beispielsweise, wenn Unternehmen selbst genau wissen, unter welchen ökologischen und sozialen Bedingungen ihre Produkte hergestellt werden, aber Konsument*innen keinen Einblick in die Produktionsbedingungen haben (vgl. King et al. 2012). Eine weitere Ausprägung von Marktversagen sind externe Effekte oder Externalitäten. Externe Effekte sind positive oder negative Auswirkungen ökonomischer Entscheidungen auf unbeteiligte Dritte - beispielsweise, wenn die Folgen umweltschädlichen Verhaltens nicht von deren Verursacher*in, sondern von Unbeteiligten getragen werden (vgl. Buchanan/ Stubblebine 1962; King et al. 2012).

Wenn Marktversagen auftritt und nationale oder transnationale Regulatoren nicht in der Lage sind, dieses Marktversagen zu beheben und die betreffenden Menschenrechte zu schützen, stehen laut den Vertreter*innen dieses normativen Ansatzes die Unternehmen in der Verantwortung. Jene Unternehmen, deren Geschäftstätigkeit in Zusammenhang mit Menschenrechtsfragen steht, haben laut diesen Autor*innen in solchen Situationen die Pflicht, sich für die betreffenden Menschenrechte einzusetzen. Diese Pflicht zum Schutz der Menschenrechte beinhaltet negative (Vermeidung von Menschenrechtsverletzungen) und positive (Beitrag zum Schutz von Menschenrechten) Verpflichtungen (vgl. de George 2005; Kobrin 2009). Nachdem Unternehmen gleichzeitig für ihr eigenes wirtschaftliches Überleben sorgen müssen, können sie oft nicht alleine handeln. Daher müssen Unternehmen für einen fairen Wettbewerb sorgen, indem sie beispielsweise gemeinsam mit anderen Akteuren neue Spielregeln für den Markt definieren, unter denen der Schutz der betreffenden Menschenrechte sichergestellt ist. Laut den Proponent*innen dieses wirtschaftsethischen Ansatzes sollen Unternehmen sich an solchen Collective- 
Action-Prozessen beteiligen, um ihr eigenes wirtschaftliches Überleben zu sichern und gleichzeitig ihrer Pflicht zur Einhaltung der Menschenrechte nachzukommen (vgl. de George 2005; Kobrin 2009).

Vertreter*innen des Ordonomics-Ansatzes sehen Unternehmen ebenfalls, wie Vertreter*innen des Corporate-Citizen-Ansatzes, als politische Akteure. Allerdings versuchen Erstere, Moral in ein ökonomisches Konstrukt zu integrieren (vgl. Pies et al. 2009; Pies et al. 2010/ 2014). Im Ordonomics-Ansatz werden drei Ebenen - oder soziale Arenen - unterschieden: Basisspiel, Metaspiel und Meta-Metaspiel. Im Basisspiel, der Wirtschaft, sind Unternehmen als Bereitsteller von Gütern und Dienstleistungen tätig. Sie interagieren hier mit ihren Stakeholdern, um Wert zu schaffen und Gewinne zu erzielen. Diese Austauschprozesse können zu unterschiedlichen positiven und negativen externen Effekten führen. Diese spiegeln jedoch laut den Proponent*innen des Ordonomics-Ansatzes nicht die Intentionen der einzelnen Akteure wider, sondern sind nicht-intendierte Folgen aus den Interaktionen im Basisspiel, die von den institutionellen Rahmenbedingungen abhängig sind. Im Metaspiel, der Politik, werden die Rahmenbedingungen für das Basisspiel geschaffen und verändert. Im Meta-Metaspiel, der Öffentlichkeit, kommt es zur öffentlichen Meinungsbildung, von der wiederum die Politik geprägt wird. Laut den OrdonomicsVertreter*innen können sich Unternehmen als Corporate Citizens abseits des Basisspiels auch im Metaspiel, also Regulierungsprozessen, und im Meta-Metaspiel, also Regelfindungsdiskursen, einbringen - und sie sollten dies in ihrem Eigeninteresse auch tun (vgl. Pies et al. 2009). Für eine Änderung der Spielregeln des Basisspiels, die im Metaspiel ausverhandelt wird, ist die Erreichung eines Konsenses unter den beteiligten Akteuren notwendig. Die Autor*innen argumentieren, dass eine Einigung über neue Spielregeln nur dann zustandekommt, wenn alle Akteure - als rational handelnde Individuen - diese für sich persönlich als vorteilhaft beurteilen. Ein Anspruch von Ordonomics ist es, durch Veränderungen des Diskurses im Meta-Metaspiel sowie durch Veränderungen der institutionellen Rahmenbedingungen im Metaspiel die postulierte Dualität zwischen Moral und Verfolgung des unternehmerischen Eigeninteresses aufzulösen. Das Bestreben des Ordonomics-Ansatzes ist demnach, als Orientierungsrahmen für die Schaffung von Win-Win-Lösungen zu dienen, die Unternehmen die Möglichkeit geben, wirtschaftlich erfolgreich und gleichzeitig gesellschaftlich nutzenstiftend zu sein. Ob der Ordonomics-Ansatz als normatives Framework gesehen werden kann, ist nicht unumstritten, da er moralische Fragen in einem auf Rational Choice beruhenden Win-Win-Konzept aufzulösen versucht. 


\section{Instrumentelle und institutionelle Gründe für Collective Action}

Welche Erklärungsansätze existieren - über die normativen Gründe hinaus dafür, dass Unternehmen selbst aktiv werden und mittels Collective-ActionInitiativen gesellschaftlich relevante Probleme lösen? Aus der klassischen ökonomischen „Rational Choice“-Perspektive wird argumentiert, dass es eine rationale Entscheidung eines Unternehmens ist, sich an einer Collective-ActionInitiative zu beteiligen, sobald die unternehmerischen Vorteile aus der Beteiligung gegenüber den Kosten überwiegen (vgl. King et al. 2012; Olson 2002/1965; Potoski/ Prakash 2013). Die Vorteile der Beteiligung an einer solchen Initiative sind entweder direkt betriebswirtschaftlich wirksam - wie beispielsweise Effizienzgewinne, Informationszugang oder Kostensenkungen oder sie bestehen darin, dass bestimmte unternehmerische Risiken - wie Reputationsrisiken oder Lieferkettenrisiken - gesenkt werden können.

Nach dieser Argumentationslinie beteiligen sich Unternehmen an Collective Action zur Lösung sozialer oder ökologischer Probleme, weil sie sich kurz- oder langfristige Vorteile für ihr Unternehmen daraus erwarten. Bis zu einem gewissen Grad scheint die Beteiligung eines Unternehmens an Collective Action also im Einklang mit dessen betriebswirtschaftlichen Zielen zu stehen (vgl. Dashwood 2014; Vogel 2008).

Die existierende Forschung zu Collective-Action-Initiativen zeigt, dass Unternehmen sich teilweise proaktiv - also aus ihrem eigenen Antrieb heraus - und teilweise reaktiv - also in Reaktion auf externen Druck und akute Probleme - an solchen Initiativen beteiligen. Die wichtigsten betriebswirtschaftlichen Gründe für das Engagement in Collective Action, die in der bestehenden Literatur identifiziert werden konnten, werden nachfolgend vorgestellt. Zur besseren Übersicht teilen wir die Gründe in drei Kategorien ein: Reputationsgründe, Regulierungsgründe und operative Gründe.

Reputationsgründe - also das Bestreben eines Unternehmens, seine Reputation in den Augen relevanter Stakeholder oder der allgemeinen Öffentlichkeit zu pflegen oder zu schützen - werden in der Literatur häufig als Erklärung für die Entstehung von Collective-Action-Initiativen genannt (vgl. Bernstein/ Cashore 2007; Chrun et al. 2016; Delmas/ Montes-Sancho 2010; Fransen 2012; Haufler 2001; Vogel 2010). Die Reputation eines Unternehmens ist dabei nicht unabhängig von der Reputation seiner Mitbewerber*innen: In der Reputationsforschung wird die Unternehmensreputation in zwei Dimensionen eingeteilt: die primäre Reputation, die von einem Unternehmen direkt beeinflusst werden kann, und die sekundär-abgeleitete Reputation, die von der Branchenreputation abhängt (vgl. Eisenegger 2005). Ein einzelnes ,schwarzes Schaf" innerhalb einer Branche kann das Ansehen der gesamten Branche beschädigen und mittels „Spillover"-Effekten wird dadurch auch die Reputation 
von Unternehmen beschädigt, die sich nichts zu Schulde kommen ließen (vgl. Barnett/ King 2008; Chrun et al. 2016; Lenox 2006; Potoski/ Prakash 2013).

Einige Unternehmen beteiligen sich proaktiv an Collective-Action-Initiativen, um ihre Reputation zu stärken. Firmen, die sich bereits als Pioniere in Hinblick auf soziale oder ökologische Themen sehen, engagieren sich beispielsweise in kollektiven Initiativen, um ihren Stakeholdern zu signalisieren, dass sie sich verantwortungsbewusster als einige ihrer Mitbewerber*innen verhalten (vgl. Haufler 2001; Lenox 2006; Saurwein 2011; Wotruba 1997). Einige Autor*innen schlagen zudem vor, dass neue Industrien - wie jene der Nanotechnologie - sich proaktiv an Collective Action beteiligen und in einen offenen Dialog über Chancen und Risiken der Technologie treten sollten, um von Beginn an ein Vertrauensverhältnis mit relevanten Stakeholdern und der Öffentlichkeit aufzubauen (vgl. Balbus et al. 2006; Bowman/ Hodge 2009).

Häufig wird in der Literatur argumentiert, dass Unternehmen sich reaktiv an kollektiven Initiativen beteiligen, um die Unternehmens- oder die Branchenreputation vor akuten Reputationsproblemen zu schützen. Die Reputation eines Unternehmens oder einer Branche kann auf unterschiedliche Weise gefährdet werden: (1) gezielte Kampagnen zivilgesellschaftlicher Organisationen (häufig verbunden mit ,naming and shaming“-Taktiken), (2) öffentliche Skandale oder Unfälle (beispielsweise das Rana-Plaza-Unglück in Bangladesh, für das die globale Textilindustrie verantwortlich gemacht wurde), (3) investigative und kritische Medienberichte oder (4) die Einführung eines Gütesiegels durch zivilgesellschaftliche Organisationen, das die öffentliche Aufmerksamkeit auf ein bestimmtes Problem lenkt (vgl. Barnett/ King 2008; Bartley 2003; Baumann-Pauly et al. 2017; Dashwood 2014; Turcotte et al. 2014).

Auch die folgende Kategorie an Gründen nimmt in der Literatur eine zentrale Rolle ein: Regulierungsgründe. In der Literatur werden verschiedene Wege beschrieben, auf denen Unternehmen mit Gesetzgebern interagieren: Unternehmen versuchen teilweise, durch die Teilnahme an Collective-ActionInitiativen (1) die Schaffung neuer Gesetze oder die Verschärfung bestehender Regulierungen gänzlich zu verhindern (vgl. Auld et al. 2015; Conley/Williams 2011; Héritier/ Eckert 2009), (2) den Zeitpunkt für die Schaffung neuer Regulierungen zu verschieben (vgl. Bowman/ Hodge 2009; Young 2013) oder (3) den Inhalt und die Stringenz neuer Regulierungen zu beeinflussen (Bowman/ Hodge 2009; Gamper-Rabindran/ Finger 2013). Der Gesetzgeber kann andererseits auch Unternehmen dazu bewegen, sich an kollektiven Initiativen zu beteiligen - entweder (4) durch positive Anreize (Balzarova/ Castka 2012; Engert 2010) oder (5) indem er den Unternehmen explizit mitteilt, dass neue Regulierungen geschaffen würden, falls die Unternehmen nicht imstande wären, das betreffende Problem selbst zu lösen (vgl. Baron 2014; Dawson/ Segerson 2008; Lyon/ Maxwell 2003). 
Wenn Unternehmen sich an kollektiven Initiativen beteiligen, bevor überhaupt politische Debatten über das betreffende Problem entstanden - beispielsweise, um rechtliche Unsicherheiten zu reduzieren -, ist das Engagement als proaktiv einzustufen. Deutlich häufiger wird in der Literatur allerdings argumentiert, dass Unternehmen reaktiv - als Reaktion auf politische Debatten und bestehenden Regulierungsdruck - auf Collective Action setzen (vgl. Dashwood 2014; Dawson/ Segerson 2008; Labatt/ Maclaren 1998). Héritier und Eckert (2009) untersuchten beispielsweise die Entstehung von Selbstregulierungsinitiativen in der Papier- und der PVC-Industrie Deutschland, Großbritanniens und Europas. Bis auf eine Ausnahme ging der Entstehung der Initiativen in allen Fällen ein plausibler und konkreter politischer Regulierungsdruck voraus.

Zusätzlich zu den zwei erwähnten Kategorien - Reputation und Regulierung - lassen sich in der Literatur einige Faktoren identifizieren, die in direktem Zusammenhang zur operativen Tätigkeit von Unternehmen stehen. Die Beteiligung an einer kollektiven Initiative kann einerseits unmittelbare unternehmerische Vorteile mit sich bringen und andererseits dabei helfen, bestimmte Risiken zu minimieren.

$\mathrm{Zu}$ den wesentlichen operativen Vorteilen zählen: Informationsaustausch innerhalb der Initiative (vgl. Acutt et al. 2004; Delmas/ Terlaak 2001), gesteigertes Innovationspotential (vgl. Bloomfield 2012; Delmas/ Terlaak 2001), Effizienzsteigerungen im Unternehmen (vgl. Acutt et al. 2004; Gereffi/ Lee 2016; Labatt/ Maclaren 1998) und Kosteneinsparungen (vgl. Bernstein/ Cashore 2007; Delmas/ Terlaak 2001; Pattberg 2005). Gelegentlich wird in der Literatur auch angeführt, dass Unternehmen sich aus ihrem Engagement in einer Collective-Action-Initiative First-Mover-Vorteile erwarten (vgl. Dashwood 2014; Fransen/ Conzelmann 2015; Wotruba 1997). Diese Vorteile entstehen beispielsweise, wenn in einer Collective-Action-Initiative ein freiwilliger Umweltstandard geschaffen wird, der einige Zeit später vom Gesetzgeber inhaltlich übernommen und in ein Gesetz umgewandelt wird, das für alle Unternehmen der Branche bindend ist. Jene Unternehmen, die sich bereits als Pioniere an der Schaffung des freiwilligen Standards beteiligen, haben in diesem Fall einen zeitlichen Vorsprung und mehr inhaltliche Gestaltungsmacht im Vergleich zu unbeteiligten Unternehmen vgl. (Dashwood 2014). Einige Autor*innen meinen auch, dass sich Unternehmen an kollektiven Initiativen beteiligen, um bestimmte Risiken für ihr Geschäft zu reduzieren. Die Beteiligung an kollektiven Initiativen ist für manche Unternehmen ein Weg, um mit Risiken innerhalb der Lieferkette umzugehen (vgl. Amekawa 2009; Fiorino/ Bhan 2016; Fulponi 2006; Zeyen et al. 2016). Unilever Deutschland war beispielsweise in den 1990er Jahren - gemeinsam mit dem Worldwide Fund for Nature (WWF) - maßgeblich an der Schaffung des Fischerei-Gütesiegels „Marine Stewardship Council“" (MSC) beteiligt. Einer der wichtigsten Gründe für 
Unilever war die Sorge, dass ein weiterer Rückgang der Fischbestände längerfristig das Geschäftsmodell des Konzerns bedrohen könnte (vgl. Hamprecht 2005). Häufig ist das Engagement eines Unternehmens in einer Collective-Action-Initiative auch eine Reaktion auf die Wünsche wichtiger Stakeholder wie Mitarbeiter*innen, Kund*innen oder Investor*innen (vgl. Acutt et al. 2004; Baines 2010; Baumann-Pauly et al. 2017; Conley/ Williams 2011; Reinecke et al. 2012). Beispielsweise wurde die Global Network Initiative von der Informationstechnologieindustrie ins Leben gerufen, weil große amerikanische Technologiefirmen hohen Druck seitens Usern, Mitarbeiter*innen, Investor*innen, Gesetzgebern und der Öffentlichkeit verspürten (vgl. BaumannPauly et al. 2017).

Die vorgestellten betriebswirtschaftlichen Gründe tragen zum Verständnis des Phänomens bei, erklären jedoch die Entstehung von Collective-Action-Initiativen nicht zur Gänze. An dieser Stelle ist es hilfreich, eine zusätzliche Perspektive einzunehmen: Neo-institutionalistische Ansätze gehen davon aus, dass Initiativen dieser Art stets in einen institutionellen Kontext eingebettet sind, aus dem sie entstehen und an den sie sich anpassen (vgl. Bartley 2007; Fransen 2013). Diesen Ansätzen zufolge entstehen Collective-Action-Initiativen nicht zwingend aus einer betriebswirtschaftlichen Entscheidung rationaler Akteure heraus, sondern können beispielsweise auch ungeplant und prozesshaft wachsen, wobei weitere Faktoren wie Vertrauen, regelmäßige informelle Kontakte zwischen den Akteuren und geteilte Wertvorstellungen in den Vordergrund rücken (vgl. Bartley 2007; Fransen 2012; Sloan/ Oliver 2013). Fransen und Burgoon (2014) erläutern beispielsweise, dass häufiger informeller Kontakt zwischen Unternehmensvertreter*innen und Vertreter*innen zivilgesellschaftlicher Organisationen die Wahrscheinlichkeit dafür erhöht, dass Unternehmen sich kollektiven Initiativen mit breiter Stakeholderinklusion anschließen. Dashwood (2014) hat den International Council on Mining and Metals (ICMM) in einer Fallstudie untersucht und festgestellt, dass RationalChoice-Faktoren - wie Reputationsrisiken oder Regulierungsdruck - zwar eine wesentliche Rolle spielten, aber keine Erklärung für den Zeitpunkt der Entstehung der Initiative liefern. Sie stützt sich auf das Konzept der critical junctures (kritischen Zeitpunkte) aus dem historischen Institutionalismus und argumentiert, dass mehrere Entwicklungen ungefähr zeitgleich stattfanden, in einem kritischen Punkt kulminierten und auf diese Weise die Manager*innen der weltweit größten Bergbauunternehmen dazu brachten, gemeinsam tätig zu werden. 


\section{Der passende Zeitpunkt für Collective Action}

Wann sollten sich Unternehmen nun an einem Collective-Action-Prozess beteiligen? Die Unternehmensethik liefert Argumente dafür, dass Unternehmen eine Verpflichtung haben, die über die Einhaltung von Gesetzen hinausgeht und die Beteiligung an Collective-Action-Initiativen zur Lösung konkreter gesellschaftlicher und ökologischer Probleme umfasst. Zusätzlich kann die Beteiligung an Initiativen dieser Art häufig auch aus einer betriebswirtschaftlichen Logik gerechtfertigt werden, wenn ein Unternehmen aus seiner Beteiligung betriebswirtschaftliche Vorteile zieht oder nachteilige Entwicklungen verhindern kann. Außerdem kann Collective Action in vielen Bereichen als eine Form systemischen Risikomanagements verstanden werden. Um den richtigen Zeitpunkt für Collective Action zu erkennen, scheint es sinnvoll, die eingangs erwähnten gesellschaftlichen und ökologischen Herausforderungen aus einer Risikomanagement-Perspektive zu sehen. Donaldson und Schoemaker (2013: 33-34) haben eine Reihe von Frühwarnsignalen für systemische Risiken identifiziert, die ein gemeinsames Vorgehen der betroffenen Unternehmen erfordern: Hohe Innovationsgeschwindigkeit, fehlender öffentlicher Diskurs über Risiken, geringe Anzahl an Expert*innen, schwache Regulatoren, fehlende Transparenz gegenüber Dritten, kritische Stimmen werden ignoriert. Diese Frühwarnsignale werden nachfolgend etwas detaillierter beschrieben.

- Hohe Innovationsgeschwindigkeit: Wenn Unternehmen sich in einem sehr intensiven Innovationswettbewerb befinden, können sich unnachhaltige Geschäftspraktiken rasch in der gesamten Branche verbreiten und nicht-intendierte Folgen nach sich ziehen. Beispiele dafür sind die Innovationen im Kreditvergabe- und Finanzwesen, welche die Finanzkrise 2008 auslösten, disruptive Geschäftsmodelle wie bei Uber oder AirBnB oder risikobehaftete technologische Neuerungen.

- Fehlender öffentlicher Diskurs über Risiken in einer Branche: Aus unterschiedlichen Gründen - beispielsweise die Angst der Unternehmen vor rechtlichen Folgen oder vor Reputationsverlusten oder auch gesellschaftliche Tabus - kann ein Geheimhaltungsdruck in einer Branche entstehen, der dazu führt, dass in der Öffentlichkeit keine kritische Diskussion über gesellschaftliche, ökologische oder auch betriebswirtschaftliche Risiken im Zusammenhang mit bestimmten Produkten, Geschäftspraktiken oder Geschäftsmodellen stattfindet. Donaldson und Schoemaker (2013) verweisen in diesem Zusammenhang auf die Tabakindustrie, die über Jahrzehnte eine offene Diskussion über Krebs- und Suchtrisiken verhinderte. Als weiteres Beispiel kann die Opiatkrise in den USA dienen, deren tatsächliches Ausmaß über eine längere Zeit öffentlich nicht sichtbar war, da Opiatabhängigkeit mit einem gesellschaftlichen Tabu belegt ist. 
- Geringe Anzahl an Expert*innen: Wenn eine gesamte Industrie für die Interpretation und Gestaltung neuer Technologien oder Geschäftsmodelle von ein paar wenigen hochspezialisierten Expert*innen abhängig ist, folgt daraus häufig eine Informationsasymmetrie zwischen diesen Expert*innen und jenen Personen innerhalb der Unternehmen, die für die Umsetzung verantwortlich sind.

- Schwache Regulatoren: Dem Gesetzgeber fehlen häufig Sachkompetenz und Insiderwissen, um systemische Risiken rechtzeitig erkennen und sie wirkungsvoll minimieren zu können. Häufig mangelt es auch am Willen politischer Entscheidungsträger*innen, unpopuläre Entscheidungen durchzusetzen oder als Staat in einer globalisierten Wirtschaft einen regulatorischen Alleingang zu wagen. Zudem ist der Gesetzgebungsprozess manchmal zu langsam, um eine Branche mit sehr hoher Innovationsgeschwindigkeit rechtzeitig - also vor dem Auftreten weitreichender Folgen für die beteiligten Unternehmen und die Gesellschaft - zu regulieren. Aus diesen Gründen sollten sich Unternehmen laut Donaldson und Schoemaker (2013) nicht auf den Gesetzgeber verlassen.

- Mangel an Transparenz gegenüber Dritten: Donaldson und Schoemaker (2013) beschreiben das Phänomen, dass sich in manchen Branchen bestimmte Praktiken verbreiten und zur Norm werden, die nur schwierig gegenüber Dritten gerechtfertigt werden könnten und die geeignet sind, das öffentliche Vertrauen in die Branche zu schädigen. Als Beispiele nennen sie die Kreditvergabepraktiken im Vorfeld der Hypothekenkrise in den USA oder die Vertriebspraktiken pharmazeutischer Konzerne, die zum Teil bis heute nicht-öffentliche Zahlungen an Ärzte leisten, damit diese ihre Produkte empfehlen.

- Kritische oder warnende Stimmen werden ignoriert: Donaldson und Schoemaker (2013) appellieren an Entscheidungsträger*innen in führenden Unternehmen, auf Kritik durch Whistleblower, Expert*innen, Aktivist*innen und Journalist*innen nicht defensiv zu reagieren, sondern Bedenkenträger*innen zuzuhören.

\section{Fazit}

Die drängenden gesellschaftlichen Herausforderungen - Digitalisierung, Klimawandel, Ressourcenknappheit etc. - verlangen nach einem kollektiven Engagement diverser Akteure, um bewältigt werden zu können. Es wäre als unternehmerische Bewältigungsstrategie unzureichend, sich auf den Gesetzgeber 
oder auf einzelne engagierte Akteure zu verlassen; Unternehmen als einflussreiche Akteure sollten daher versuchen, gemeinsam mit ihren Stakeholdern an Lösungen zu arbeiten.

Die normativen Gründe für Unternehmen, sich an Collective Action zu beteiligen, liegen in der Verantwortung der Unternehmen als Corporate Citizens, in der Vermeidung von Krisen, im Schutz der Umwelt, der sozialen Stabilität und der Menschenrechte. Die Partizipation an kollektiven Initiativen kann zudem in unmittelbaren unternehmerischen Vorteilen resultieren. Zusätzlich kann die Beteiligung an kollektiven Initiativen der Verhinderung systemischer Risiken dienen - und damit auch dem Schutz vor negativen betriebswirtschaftlichen Konsequenzen wie Reputationsverlusten sowie allzu restriktiven staatlichen Regulierungen für ganze Industrien. Um Collective Action zu ermöglichen, ist ein langfristig orientiertes Risikomanagement erforderlich, das in der Lage ist, systemrelevante Risiken zu erkennen - auch, wenn ihre Eintrittswahrscheinlichkeit gering ist. Außerdem ist es hierfür notwendig, dass Unternehmen, die einander ansonsten als Konkurrenten begegnen, miteinander - und gegebenenfalls mit weiteren Stakeholdern - in einen offenen und transparenten Dialog treten, um gemeinsam wirksame Maßnahmen entwickeln zu können. Um zu erkennen, wann der richtige Zeitpunkt ist, ein Problem auf kollektiver Ebene zu behandeln, können sich Entscheidungsträger*innen an einer Reihe von Frühwarnsignalen orientieren.

\section{Literatur}

Acutt, N. J. /Medina-Ross, V. /O'Riordan, T. (2004): Perspectives on corporate social responsibility in the chemical sector. A comparative analysis of the Mexican and South African cases. In: Natural Resources Forum 28, 4, S. 302-316.

Aguinis, H./Glavas, A. (2012): What we know and don't know about corporate social responsibility. A review and research agenda. In: Journal of Management 38, 4, S. 932-968.

Amekawa, Y. (2009): Reflections on the growing influence of good agricultural practices in the global south. In: Journal of Agricultural \& Environmental Ethics 22, 6, S. 531-557.

Auld, G./Balboa, C./Bernstein, S./Cashore, B./Delmas, M./Young, O. (2009): The emergence of non-state market-driven (NSDM) global environmental governance. Governance for the Environment: New Perspectives. S. 183. Cambridge: Cambridge University Press.

Auld, G./Renckens, S./Cashore, B. (2015): Transnational private governance between the logics of empowerment and control. In: Regulation \& Governance 9, 2, S. 108124.

Bäckstrand, K. (2012): Are partnerships for sustainable development democratic and legitimate? In: Pattberg, P./Biermann, F./Chan, S./Mert, A. (Hrsg.): Public-private 
partnerships for sustainable development. Emergence, influence and legitimacy. Northampton. MA: Edward Elgar. S. 165-182.

Baines, R. (2010): Quality and safety standards in food supply chains. In: Mena, C./ Stevens, G. (Hrsg.): Delivering performance in food supply chains. Oxford/ Cambridge/ New Dehli: Woodhead Publishing Ltd., S. 303-323.

Balbus, J. M./Florini, K./Denison, R. A./Walsh, S. A. (2006): Getting it right the first time. Developing nanotechnology while protecting workers, public health, and the environment. In: Mehlman, M.A./Soffritti, M./Landrigan, P./Bingham E./Belpoggi F. (Hrsg.): Living in a chemical world. framing the future in light of the past vol. 1076. Wiley-Blackwell. S. 331-342.

Balzarova, M. A./Castka, P. (2012): Stakeholders' influence and contribution to social standards development. The case of multiple stakeholder approach to ISO 26000 development. In: Journal of Business Ethics 111, 2, S. 265-279.

Barnett, M. L./King, A. A. (2008): Good fences make good neighbors. A longitudinal analysis of an industry self-regulatory institution. In: Academy of Management Journal 51, 6, S. 1150-1170.

Baron, D. P. (2014): Self-regulation in private and public politics. In: Quarterly Journal of Political Science 9, 2, S. 231-267.

Bartley, T. (2003): Certifying forests and factories. States, social movements, and the rise of private regulation in the apparel and forest products fields. In: Politics \& Society 31, 3, S. 433-464.

Bartley, T. (2007): Institutional emergence in an era of globalization. The rise of transnational private regulation of labor and environmental conditions. In: American Journal of Sociology 113, 2, S. 297-351.

Baumann-Pauly, D./Nolan, J./van Heerden, A./Samway, M. (2017): Industry-specific multi-stakeholder initiatives that govern corporate human rights standards. In: Journal of Business Ethics 143, 4, SI, S. 771-787.

Bernstein, S./Cashore, B. (2007): Can non-state global governance be legitimate? An analytical framework. In: Regulation \& Governance 1, 4, S. 347-371.

Bloomfield, M. J. (2012): Is forest certification a hegemonic force? The FSC and its challengers. In: Journal of Environment \& Development 21, 4, S. 391-413.

Bowman, D. M./Hodge, G. A. (2009): Counting on codes. An examination of transnational codes as a regulatory governance mechanism for nanotechnologies. In: Regulation \& Governance 3, 2, S. 145-164.

Brammer, S./Jackson, G./Matten, D. (2012): Corporate social responsibility and institutional theory. New perspectives on private governance. In: Socio-Economic Review 10. S. 3-28.

Buchanan, J. M./Stubblebine, W. C. (1962): Externality. In: economica 29, 116, S. 371384.

Cashore, B. (2002): Legitimacy and the privatization of environmental governance. How non-state market-driven (NSMD) governance systems gain rule-making authority. In: Governance-An International Journal of Policy and Administration 15, 4, S. 503-529.

Cashore, B./Auld, G./Bernstein, S./McDermott, C. (2007): Can non-state governance 'ratchet up' global environmental standards? Lessons from the forest sector. In: Review of European Community \& International Environmental Law 16, 2, S. 158172. 
Chrun, E./Dolsak, N./Prakash, A. (2016): Corporate environmentalism. Motivations and mechanisms. In: Gadgil, A./Gadgil, T. P. (Hrsg.): Annual review of environment and resources 41 , S. 341-362.

Conley, J. M./Williams, C. A. (2011): Global banks as global sustainability regulators?. The equator principles. In: Law \& Policy 33, 4, S. 542-575.

Cooper, J. (2018): Congress and the decline of public trust. New York: Routledge.

Cortez, N. (2014): Regulating disruptive innovation. Berkeley Tech. LJ 29, S. 175.

Dashwood, H. S. (2014): Sustainable development and industry self-regulation. Developments in the global mining sector. In: Business \& Society 53, 4, SI, S. 551-582.

Dawson, N. L./Segerson, K. (2008): Voluntary agreements with industries. Participation incentives with industry-wide targets. In: Land Economics 84, 1, S. 97-114.

De Bandt, O./Hartmann, P. (2000): Systemic risk. A survey. ECB Working Paper no. 35. Frankfurt a. M.

De George, R. T. (2005): Intellectual property and pharmaceutical drugs. An ethical analysis. In: Business Ethics Quarterly 15, 4, S. 549-575.

De los Reyes, G./Scholz, M./Smith, N. C. (2017): Beyond the "win-win". Creating shared value requires ethical frameworks. In: California Management Review 59, 2, S. 142-167.

Delmas, M. A./Montes-Sancho, M. J. (2010): Voluntary agreements to improve environmental quality. Symbolic and substantive cooperation. In: Strategic Management Journal 31, 6, S. 575-601.

Delmas, M. A./Terlaak, A. K. (2001): A framework for analyzing environmental voluntary agreements. In: California Management Review 43, 3, S. 44-63.

Donaghey, J./Reinecke, J. (2018): When industrial democracy meets corporate social responsibility - A comparison of the Bangladesh Accord and Alliance as responses to the Rana Plaza Disaster. In: British Journal of Industrial Relations 56, 1, S. 14 42.

Donaldson, T./Schoemaker, P. J. H. (2013): Self-inflicted industry wounds. Early warning signals and pelican gambits. In: California Management Review 55, 2, S. 24 45.

Eisenegger, M. (2005): Reputation in der Mediengesellschaft: Konstitution-Issues Monitoring-Issues Management. Wiesbaden: Springer-Verlag.

Engert, A. (2010): Transnational hedge fund regulation. In: European Business Organization Law Review 11, 3, S. 329-378.

Fiorino, D. J./Bhan, M. (2016): Supply chain management as private sector regulation. What does it mean for business strategy and public policy? In: Business Strategy and the Environment 25, 5, S. 310-322.

Fransen, L. (2012): Multi-stakeholder governance and voluntary programme interactions: Legitimation politics in the institutional design of corporate social responsibility. In: Socio-Economic Review 10, 1, SI, S. 163-192.

Fransen, L. (2013): The Embeddedness of Responsible Business Practice: Exploring the Interaction Between National-Institutional Environments and Corporate Social Responsibility. In: Journal of Business Ethics 115, 2, S. 213-227.

Fransen, L./Burgoon, B. (2014): Privatizing or socializing corporate responsibility. Business participation in voluntary programs. In: Business \& Society 53, 4, SI, S. 583-619. 
Fransen, L./Conzelmann, T. (2015): Fragmented or cohesive transnational private regulation of sustainability standards? A comparative study. In: Regulation \& Governance 9, 3, S. 259-275.

Fulponi, L. (2006): Private voluntary standards in the food system. The perspective of major food retailers in OECD countries. In: Food Policy. 31, 1, S. 1-13.

Gamper-Rabindran, S./Finger, S. R. (2013): Does industry self-regulation reduce pollution? Responsible Care in the chemical industry. In: Journal of Regulatory Economics 43,1 , S. $1-30$.

George, G./Howard-Grenville, J./Joshi, A./Tihanyi, L. (2016): Understanding and Tackling Societal Grand Challenges through Management Research. In: Academy of Management Journal 59, 6, S. 1880-1895.

Gereffi, G./Lee, J. (2016): Economic and social upgrading in global value chains and industrial clusters. Why governance matters. In: Journal of Business Ethics 133, 1, S. $25-38$.

Global Footprint Network (2018): Has humanity's ecological footprint reached its peak?, https://www.footprintnetwork.org/2018/04/09/has_humanitys_ecological_footprint_reached_its_peak/. [Zugriff: 09.04.2018].

Guttentag, D. (2015): Airbnb. Disruptive innovation and the rise of an informal tourism accommodation sector. In: Current issues in Tourism 18, 12, S. 1192-1217.

Habermas, J. (1996): Between facts and norms. Cambridge. MA: MIT Press.

Habermas, J./Pensky, M. (2001): The postnational constellation. Political essays. Cambridge/ UK: Polity Press.

Hamprecht, J. (2005): Sustainable purchasing strategy in the food industry. Dissertation of the University of St. Gallen. Bamberg: Difo Druck GmbH.

Hanitzsch, T./van Dalen, A./Steindl, N. (2018): Caught in the Nexus. A comparative and longitudinal analysis of public trust in the press. In: The International Journal of Press/Politics 23, 1, S. 3-23.

Haufler, V. (2001): A public role for the private sector. Industry self-regulation in a global economy 1. Auflage. Washington DC: Carnegie Endowment for International Peace.

Héritier, A./Eckert, S. (2009): Self-regulation by associations. Collective action problems in European environmental regulation. In: Business and Politics 11, 1, S. 122.

Huxham, C./Macdonald, D. (1992): Introducing collaborative advantage. Achieving inter-organizational effectiveness through meta-strategy. In: Management Decision 30, 3, S. 50-56.

IPCC (2018): Global warming of $1.5^{\circ} \mathrm{C}$. Sixth assessment report (AR6).

Jahdi, K. S./Acikdilli, G. (2009): Marketing communications and corporate social responsibility (CSR). Marriage of convenience or shotgun wedding? In: Journal of Business Ethics 88, 1, S. 103-113.

King, A./Prado, A. M./Rivera, J. (2012): Industry self-regulation and environmental protection. In: Bansal, P. / Hoffman, A.J. (Hrsg.): The Oxford Handbook of Business and the Natural Environment. Oxford: Oxford University Press.

King, A. A./Lenox, M. J. (2000): Industry self-regulation without sanctions. The chemical industry's responsible care program. Academy of Management Journal 43, 4, S. 698-716. 
Kobrin, S. J. (2009): Private political authority and public responsibility. Transnational politics, transnational firms, and human rights. In: Business Ethics Quarterly 19, 3, S. 349-374.

Kolk, A./van Tulder, R. (2005): Setting new global rules? In: Transnational Corporations 14,3 , S. 1-17.

Labatt, S./Maclaren, V. W. (1998): Voluntary corporate environmental initiatives. A typology and preliminary investigation. In: Environment and Planning C-Government and Policy 16, 2, S. 191-209.

Lenox, M. J. (2006): The role of private decentralized institutions in sustaining industry self-regulation. In: Organization Science 17, 6, S. 677-690.

Lock, I./Seele, P. (2016): The credibility of CSR (corporate social responsibility) reports in Europe. Evidence from a quantitative content analysis in 11 countries. Bridges for a more sustainable future. Joining Environmental Management for Sustainable Universities (EMSU) and the European Roundtable for Sustainable Consumption and Production (ERSCP) conferences 122. S. 186-200.

Lyon, T. P./Maxwell, J. W. (2003): Self-regulation, taxation and public voluntary environmental agreements. In: Journal of Public Economics 87, 7-8, S. 1453-1486.

Manning, S./Reinecke, J. (2016): A modular governance architecture in-the-making. How transnational standard-setters govern sustainability transitions. In: Research Policy 45, 3, S. 618-633.

Matten, D./Crane, A. (2005): Corporate citizenship: Toward an extended theoretical conceptualization. In: Academy of Management Review 30, 1, S. 166-179.

Mena, S./Palazzo, G. (2012): Input and output legitimacy of multi-stakeholder initiatives. In: Business Ethics Quarterly 22, 3, S. 527-556.

OECD (2017): Key points of the hearing on disruptive innovation no. DAF/COMP/M(2015)1/ANN8/FINAL. Directorate for financial and enterprise affairs. Competition committee.

Olson, M. (2002/1965): Logic of collective action. Public goods and the theory of groups. 20. Auflage. Harvard University Press.

Pattberg, P. (2005): The institutionalization of private governance. How business and nonprofit organizations agree on transnational rules. In: Governance-An International Journal of Policy and Administration 18, 4, S. 589-610.

Pies, I., Beckmann, M./Hielscher, S. (2010): Value creation, management competencies, and global corporate citizenship. An ordonomic approach to business ethics in the age of globalization. In: Journal of Business Ethics 94, 2, S. 265-278.

Pies, I./Beckmann, M./Hielscher, S. (2014): The political role of the business firm. An ordonomic concept of corporate citizenship developed in comparison with the aristotelian idea of individual citizenship. In: Business \& Society 53, 2, SI, S. 226-259.

Pies, I./Hielscher, S./Beckmann, M. (2009): Moral commitments and the societal role of business. An ordonomic approach to corporate citizenship. In: Business Ethics Quarterly 19, 3, SI, S. 375-401.

Potoski, M./Prakash, A. (2013): Green clubs: Collective action and voluntary environmental programs. In: Annual Review of Political Science 16, S. 399-419.

Reinecke, J./Donaghey, J. (2015): After Rana Plaza: Building coalitional power for labour rights between unions and (consumption-based) social movement organisations. In: Organization Studies 22, 5, S. 720-740. 
Reinecke, J./Manning, S./von Hagen, O. (2012): The emergence of a standards market. Multiplicity of sustainability standards in the global coffee industry. In: Organization Studies 33, 5-6, S. 791-814.

Saurwein, F. (2011): Regulatory choice for alternative modes of regulation. How context matters. In: Law \& Policy 33, 3, S. 334-366.

Scherer, A. G./Palazzo, G./Baumann, D. (2006): Global rules and private actors. Toward a new role of the transnational corporation in global governance. In: Business Ethics Quarterly 16, 4, S. 505-532.

Scherer, A. G./Palazzo, G./Matten, D. (2014): The business firm as a political actor. A new theory of the firm for a globalized world. In: Business \& Society 53, 2, S. $143-$ 156.

Scherer, A. G./Rasche, A./Palazzo, G./Spicer, A. (2016): Managing for political corporate social responsibility. New challenges and directions for PCSR 2.0. In: Journal of Management Studies 53, 3, S. 273-298.

Sloan, P. / Oliver, D. (2013): Building trust in multi-stakeholder partnerships. Critical emotional incidents and practices of engagement. In: Organization Studies 34, 12 , S. $1835-1868$.

Steffen, W./Richardson, K./Rockström, J./Cornell, S. E./Fetzer, I./Bennett, E. M./Biggs, R./Carpenter, S. R./Vries, W. de/Wit, C. A. de (2015): Planetary boundaries. Guiding human development on a changing planet. In: Science 347, 6223, 1259855.

Steinmann, H./Löhr, A. (1996): A republican concept of corporate ethics Europe's Challenges. Wiesbaden: Springer. S. 21-60.

Steinmann, H./Scherer, A. G. (1998): Corporate ethics and global business. Philosophical considerations on intercultural management. In: Kumar, B./ Steinmann, H. (Hrsg.): Ethics in international management. Berlin/New York: Walter de Gruyter, S. $13-46$.

Steinmann, H./Scherer, A. G. (2000): Corporate ethics and management theory. Contemporary economic ethics and business ethics. Wiesbaden: Springer, S. 148-192.

Stringham, E. (2015): Private governance: Creating order in economic and social life. Oxford: Oxford University Press.

Turcotte, M.-F./Reinecke, J./den Hond, F. (2014): Explaining variation in the multiplicity of private social and environmental regulation. A multi-case integration across the coffee, forestry and textile sectors. In: Business and Politics 16, 1, S. 151-189.

Ulrich, P. (2000): Integrative Wirtschaftsethik. Grundlagenreflexion der ökonomischen Vernunft. Ethik und Sozialwissenschaften. In: Streitforum für Erwägungskultur 11, 4, S. 555-567.

Ulrich, P. (2002): Republikanischer Liberalismus und Corporate Citizenship. Gemeinwohl und Gemeinsinn. Zwischen Normativität und Faktizität. S. 273-291.

Verantwortungspartner.de (2014): Ingolstadt. https://goo.gl/a9M64R. [Zugriff: 09.04.2018].

Vogel, D. (2008): Private global business regulation. Annual Review of Political Science 11. S. 261-282.

Vogel, D. (2010): The private regulation of global corporate conduct. Achievements and limitations. In: Business \& Society 49, 1, S. 68-87.

Wotruba, T. R. (1997): Industry self-regulation. A review and extension to a global setting. In: Journal of Public Policy \& Marketing 16, 1, S. 38-54. 
Young, K. (2013): Financial industry groups' adaptation to the post-crisis regulatory environment. Changing approaches to the policy cycle. In: Regulation \& Governance 7, 4, S. 460-480.

Zeyen, A./Beckmann, M./Wolters, S. (2016): Actor and institutional dynamics in the development of multi-stakeholder initiatives. In: Journal of Business Ethics 135, 2, S. 341-360. 



\title{
Methodische Schärfung der CSR-Diskussion
}

\author{
Thomas Rusche
}

Die Unternehmung (Corporate) hat als erfolgsorientiertes Sozialsystem (Social) eine Verantwortung (Responsibility) für soziale und ökologische Ziele, die über die Gewinnerzielung hinausgeht. Dieses Postulat der CSR-Bewegung, deren Ursprünge in den 1950er Jahren liegen (Aßländer 2011: 171f.; Hardtke 2010: 26; Hansen/Schrader, 2005: 375; Carroll 1999: 268f.; Carroll 2009: 19f.; Hiß 2006: 22f.), steht offensichtlich quer zum Paradigma der Gewinnmaximierung und ,überschreite( $\mathrm{t}$ ) die Grenzen des klassischen ökonomischen Erkenntnisobjekts" (Hansen/Schrader 2005: 382). ${ }^{1}$ Das terminologische Ausbuchstabieren (vgl. Rusche 2009: 55f.), theoretische Durchdringen und praxeologische Konkretisieren des Konzeptes der CSR für die unterschiedlichen Anwendungsfelder in der Unternehmensführung bedarf m. E. einer transdisziplinären methodischen Schärfung. ${ }^{2}$

„Die wissenschaftliche Auseinandersetzung mit CSR erfordert nicht nur eine Überwindung betriebswirtschaftlicher Teildisziplinen, sondern letztlich eine Interdisziplinarität, an der sich neben Betriebswirten z. B. für anstehende sozialwissenschaftliche Fragen Soziologen und Politologen und für ökologische Folgenabschätzungen Naturwissenschaftler und Ingenieure beteiligen müssen. Nun ist interdisziplinäre Forschung an staatlichen Universitäten im deutschsprachigen Raum aufgrund relativ fester institutioneller Fakultätsgrenzen ziemlich schwerfällig" (Hansen/Schrader 2005: 382).

2 Vgl. Sammelband Gesellschaftliche Verantwortung von Unternehmen - Von der Idee der Corporate Social Responsibility zur erfolgreichen Umsetzung, herausgegeben von Arnd Hardtke und Annette Kleinfeld, der theoretisch fundierte und anwendungsorientierte Beiträge von Betriebswirten, Volkswirten, Soziologen, Sozialpädagogen, Theologen, Politologen, Umweltwissenschaftlern und Physikern umfaßt und zahlreiche praktische Anwendungsfälle in Unternehmen analysiert; vgl. Jahrestagung der Bertelsmann-Stiftung, Fachkonferenz „Gesellschaftliche Verantwortung im Mittelstand“, Berlin 21.11.2011. 


\section{Ethische Maßstabslosigkeit des ISO-Standards 26000}

Trotz oder gerade wegen der von Peter Ulrich bereits 1977 konstatierten ,ins Unüberschaubare angewachsenen 'Social Responsibility'-Literatur" (Ulrich 1977: 213) wird bis heute ein „Dschungel von Begriffsdefinition(en)“ (Hardtke 2010: 16) beklagt und gefragt: „Was ist eigentlich CSR?“ (Hardtke 2010: 16; Aßländer 2009: 28f.) Auf diese Frage geben unterschiedliche internationale Organisationen mehr oder weniger kodifizierte Antworten. ${ }^{3}$ So hat die International Organisation for Standardisation im Jahre 2010 mit dem ISO-Standard 26000 eine normierte Antwort formuliert und Orientierungshilfen für das verantwortliche Handeln von Unternehmensorganisationen verfasst, die in Abbildung 1 visualisiert werden (vgl. Aßländer 2011: 176f.; Hardtke 2010: 38; Bruton 2011: 42f.).

Als ,Kernthemen gesellschaftlicher Verantwortung' werden neben der übergreifenden ,Organisationsführung' die ,Menschenrechte', ,Arbeitsbedingungen', ,Umwelt', ,faire Betriebspraktiken', ,Konsumentenfragen', ,Einbeziehung und Entwicklung der Gesellschaft' herausgestellt, die entsprechend folgender sogenannter ,Prinzipien gesellschaftlicher Verantwortung' durch ,Identifizierung der Anspruchsgruppen' für die jeweilige Unternehmung konkretisiert werden sollen. Als solche werden benannt: „Rechenschaftspflicht, Transparenz, Ethisches Verhalten, Berücksichtigung der Interessen der Stakeholder, Achtung der Rechtstaatlichkeit, Einhaltung internationaler Verhaltensstandards, Achtung der Menschenrechte“. (ISO-26000 Standard) ${ }^{4}$ Die insbesondere für technische Normierungsfragen maßgebliche Autorität der Internationalen Organisation für Normierung veranschaulicht mit dem ISO 26000Standard zahlreiche Inhalte einer verantwortungsvollen Unternehmensführung.

Aufgrund einer unzureichenden Begriffsdefinition von Corporate Social Responsibility gelingt es ISO-26000 allerdings nicht, den oben beklagten Begriffsdschungel zu lichten. Vielmehr ist eine fehlende terminologische Trennschärfe zu beklagen. Diese zeigt sich z. B. an der umgangssprachlichen Verwendung des zentralen philosophischen Begriffs ,Prinzip“, das als letztverbindlicher formal-allgemeiner Maßstab den konkret-allgemeinen Verantwortungsfeldern der Unternehmensführung zugrunde gelegt werden soll (vgl. Apel 1988: 420).

Vgl. die Prinzipien für multinationale Unternehmen der ILO (International Labour Organization); vgl. UN Global Compact (www.unglobalcompact.com [Zugriff: 06.05.2019]); vgl. Social Accountability 8000 der SAI (Social Accountability International); vgl. Global Reporting Initiative (GRI) der CERES (Coalition of Environmentally Responsible Economies); vgl. Accountability 1000 des Institut of Social and Ethical Accountability. vgl. Hardtke 2010: 38. 
Abbildung 1: Inhaltliche und konzeptionelle Struktur der ISO-26000

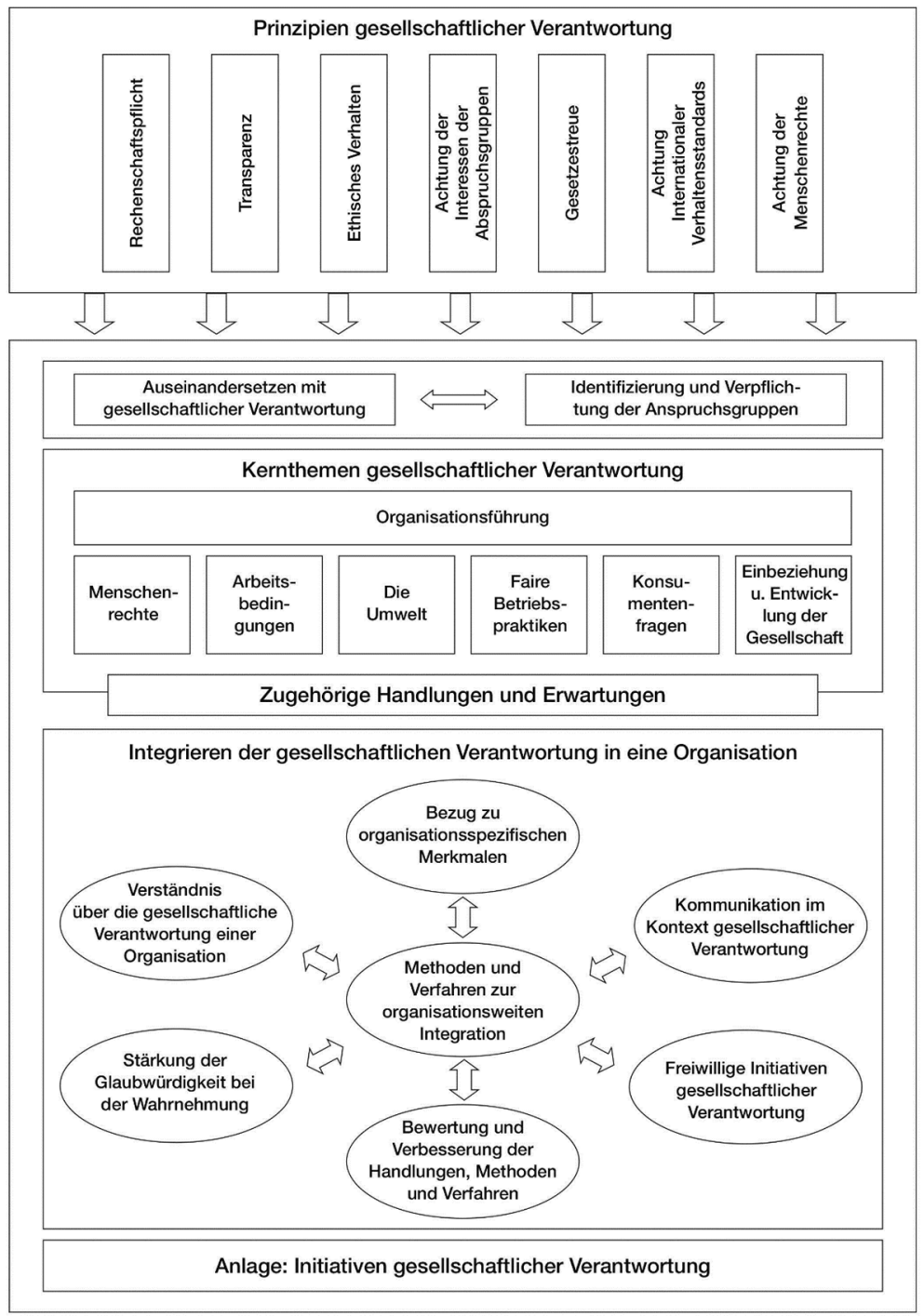

Quelle: Deutsches Institut für Normierung e. V. 2011: 12 
Zweifelsohne besitzen die von ISO-26000 explizit benannten Menschenrechte als völkerrechtliche Kodifizierung des Prinzips der Menschenwürde einen solchen erstrangigen Status eines allem anderen zugrundeliegenden bzw. übergeordneten Prinzips. Die Orientierung an den Menschenrechten soll ,ethisches Verhalten' ermöglichen. ,Ethisches Verhalten' ist aber ebenso wenig ein Prinzip wie die Achtung internationaler Verhaltensstandards, die zunächst selbst aus der Perspektive eines zugrunde gelegten ethischen Prinzips kritisch beurteilt und begründet werden müssten. Bemerkenswert ist auch die Redundanz und fehlende Trennschärfe der sogenannten Prinzipien untereinander (z. B. ,Rechenschaftspflicht' und ,Transparenz') sowie zwischen den Prinzipien und Kernthemen (z. B. Menschenrechte). Warum wird ,Umwelt' zwar als Kernthema benannt, aber kein umweltschützendes Prinzip der Zukunftsverantwortung begründet und für die Kernthemen orientierungsstiftend vorausgesetzt?

Offensichtlich fehlt es ISO-26000 an begrifflicher Klarheit, um zwischen inhaltlichen Kernthemen der Unternehmenspraxis und zu begründenden ethischen Prinzipien zu unterscheiden, mittels derer die Unternehmenspraxis verantwortungsvoll gestaltet werden könnte. An dieser Stelle vermeidet die CSRDiskussion jede methodische Begründungsanstrengung. ${ }^{5}$ Die Frage nach einer zureichenden Begründung der proklamierten Prinzipien (principium rationis sufficientis) wird also weder gestellt, noch hinreichend beantwortet. ${ }^{6}$ Vielmehr werden Kategorienfehler wie z. B. die Benennung der ,Achtung internationaler Verhaltensstandards' als erstrangiges Prinzip in Kauf genommen, wiewohl solche Standards selbst der Rechtfertigung oder der kritischen Prüfung anhand eines ethischen Prinzips bedürfen. Ohne eine kategoriale Unterscheidung von ethischen Prinzipien und konkreten Handlungsfeldern greift ein solch anwendungsorientierter Ansatz systematisch zu kurz.

Neben der fehlenden intensionalen und extensionalen begrifflichen Klarheit der CSR-Definition sowie des Kategorienfehlers, unbegründete ethische Prinzipien mit thematischen Handlungsfeldern zu vermengen, fehlt auffälliger Weise die Unterscheidung zwischen der Sphäre des Rechts und der Moral. So gelingt es ISO-26000 nicht, die moralische Verantwortung von der juristischen Verantwortung - z. B. für, Gesetzestreue' und ,Einhaltung von internationalen Standards' - zu unterscheiden. Die anzustrebende Legalität im Sinne der, Gesetzestreue' ist jedoch kategorial von der ethisch reflektierten moralischen $\mathrm{Le}$ gitimität zu unterscheiden (vgl. Abschnitt 9.1). Diese Vermengung von Recht

Küpper versucht nachvollziehbare „Strukturmuster zur Begründung von Normen des Corporate Governance-Kodex“ aufzuzeigen und leitet aus ,,allgemein akzeptierte(n) Werte(n) und Normen“, wie „Menschenwürde“ und „Wohlstandsmaximierung“ [sic!] konkrete „Normen der Corporal Governance“ ab (Küpper 2011: 209f.).

6 Aßländer und Brink beklagen in der CSR-Diskussion eine „offensichtliche Lücke“ zwischen der inhaltlichen Fülle und ,,der theoretischen Frage, wie sich eine Pflicht zur sozialen Verantwortungsübernahme ethisch begründen lässt“" (Aßländer/Brink 2008: 109). 
und Moral kennzeichnet die CSR-Diskussion seit Anbeginn (vgl. Ulrich 1977: 213f.) und kann durch ISO-26000 nicht überwunden werden.

Wenn positiv gesetztes Recht ethisch-normativ aufgeladen wird, so führt diese Gleichsetzung von Recht und Moral zu einer Konfusion von Deskription und Präskription. Historisch konkrete Rechtsnormen, z. B. solche des Arbeitsund Umweltschutzes, die bekanntlich nach Rechtsraum und Zeitperiode voneinander abweichen, können nicht ohne kritische Prüfung zur moralischen Sollensnorm erhoben werden. Die Formulierungen von ISO-26000 legen den methodischen Fehlschluss nahe, aus der Deskription von (juristischen) Normen eine (moralische) Präskription folgern zu können. Dieser übergangslose Wechsel von deskriptiver und normativer Perspektive ist fatal: Natürlich beeinflussen Faktizitäten die kritische Ausgestaltung von Sollensnormen, jedoch bedarf es einer ethischen Kritik- und Begründungsebene, auf der die faktischen Rechtsnormen, Verhaltensstandards und Branchennuancen mittels prinzipieller Maßstäbe geprüft werden können. Diese Kritik und Begründung wird von ISO-26000 nicht geleistet; auffällig ist vielmehr eine ethische Maßstabslosigkeit, eine Konfusion von Sein und Sollen, also dessen, was faktisch ist (z. B. Verhaltensstandards) und dessen, was als Sollen zu begründen wäre. Alle Bausteine von ISO-26000 scheinen gleichbedeutend zu sein (vgl. Kuhn 1991: 30) und kennzeichnen die betriebswirtschaftliche CSR-Diskussion als interdisziplinären Reparaturbetrieb ohne paradigmatische Klarheit.

\section{Paradigmatischer Konflikt von Gewinnmaximierung und CSR}

Fehlende paradigmatische Klarheit benennt Thomas Kuhn (1922-1996) als charakteristisches Merkmal der Frühzeit einer Wissenschaft:

„Beim Fehlen eines Paradigmas oder eines Kandidaten für ein Paradigma scheinen alle Tatsachen, die irgendwie zu der Entwicklung einer bestimmten Wissenschaft gehören könnten, gleichermaßen relevant zu sein. Folglich ist das Zusammentragen von Fakten in der Frühzeit eine Tätigkeit, die weit mehr dem Zufall unterliegt als die, welche die darauf folgende wissenschaftliche Entwicklung kennzeichnet. [...] Kein Wunder also, daß in den frühen Stadien der Entwicklung jeder Wissenschaft verschiedene Leute, die sich dem gleichen Bereich von Phänomenen, aber gewöhnlich nicht alle den gleichen Phänomenen gegenübersehen, sie auch auf unterschiedliche Art und Weise beschreiben und interpretieren"(Kuhn 1991: 30f.).

Eine solche methodisch unscharfe Beschreibung und teils widersprüchliche Bewertung der CSR-relevanten Phänomene charakterisiert die Paradigmenkrise der Betriebswirtschaftslehre. Beispielsweise bleibt unklar, ob CSR-Maßnahmen nur dann ergriffen werden sollen, wenn sie als Instrument der (langfristigen) Gewinnerzielung dienen oder auch für den Fall der Belastung des (Perioden-)Gewinns. 
In den 1950er Jahren hatte sich in Abkehr von der ethisch-normativen BWL ein Paradigmenwechsel vollzogen. Nicht die Verantwortung für Mensch und Gesellschaft, sondern für den Unternehmensgewinn wurde der Bezugspunkt einer neuen Generation von Betriebswirtschaftler*innen. Seitdem dominiert das Paradigma der Gewinnmaximierung das Theorie- und Lehrgebäude der BWL und prägte mehrere Forschergenerationen.

„Das Studium der Paradigmata [...] ist für den Studierenden die wichtigste Vorbereitung für die Mitgliedschaft in einer bestimmten wissenschaftlichen Gemeinschaft, in der er später arbeiten will. Da er sich dort Menschen anschließen wird, welche die Grundlagen ihres Gebietes anhand derselben konkreten Vorbilder kennengelernt haben, wird seine spätere Arbeit selten offene Meinungsverschiedenheiten über Grundprinzipien auslösen. Menschen, deren Forschung auf gemeinsamen Paradigmata beruht, sind denselben Regeln und Normen für die wissenschaftliche Praxis verbunden. Diese Bindung und die offenbare Übereinstimmung, die sie hervorruft, sind Voraussetzungen für eine normale Wissenschaft, d. h. für die Entstehung und Fortdauer einer bestimmten Forschungstradition“ (Kuhn 1991: 26). ${ }^{7}$

Auf diese Weise hat sich im Forschungs- und Lehrbetrieb der BWL die paradigmatische Ausrichtung am Gewinnmaximierungsprinzip etabliert. Seither besteht auch betriebswirtschaftliche Forschung und Praxis

„,in der Verwirklichung jener Verheißung, einer Verwirklichung, die durch Erweiterung der Kenntnis der vom Paradigma als besonders aufschlußreich dargestellten Fakten, durch Verbesserung des Zusammenspiels dieser Fakten mit den Voraussagen des Paradigmas sowie durch weiter Artikulierung des Paradigmas selbst herbeigeführt wird“ (Kuhn 191: 38).

Mit der Wahl des Paradigmas der Gewinnmaximierung erwirbt die Forschungsgemeinschaft zugleich ,ein Kriterium für die Wahl von Problemen [...], von welchen - solange das Paradigma nicht in Frage gestellt wird - vermutet werden kann, daß sie eine Lösung haben“" (Kuhn 1991: 51).

Wie bereits dargestellt, gelingt es der Betriebswirtschaftslehre entsprechend dem zielrational nicht weiter problematisierten Paradigma der Gewinnmaximierung, die notwendigen mittelrationalen Verfahren zur Steigerung des Periodengewinns für das laufende operative Tagesgeschäft einer Unternehmung zur Verfügung zu stellen. Die Fokussierung auf mittelrationale Problemstellungen erscheint als Erfolgs- und Fortschrittsgeschichte. Angesichts steigender Unternehmensgewinne und wachsender Produktivität in der Leistungserstellung, steigender Börsenwerte und Rentabilitätskennziffern erlangt das Paradigma der Gewinnmaximierung seinen bestimmenden ,Status, weil [... es] bei der Lösung einiger Probleme, welche ein Kreis von Fachleuten als brennend erkannt hat, erfolgreicher [... ist] als die [...] konkurrierenden" (Kuhn 1991: 37). Die Leistungsfähigkeit dieses Paradigmas zeigt sich in der Entwicklung mittelrationaler Instrumente zur erfolgsorientierten Ausgestaltung aller Organisationsbereiche der Unternehmung; und es ermöglicht eine spezifische 
Ausprägung des Shareholderkapitalismus, in dem die vorrangige Verantwortung der Manager*innen für den Erfolg der Anteilseigner*innen postuliert wird, der sich in steigenden Dividenden und Aktienkursen ausdrückt.

Nicht zuletzt durch den Zufluss von Drittmitteln aus Unternehmensgewinnen finanziert, expandiert die betriebswirtschaftliche Forschung mit ihrem Paradigma der Gewinnmaximierung auch in erfolgsrelevante verhaltenswissenschaftliche Bereiche. Ja, sie versucht $-\mathrm{z}$. B. mittels neurophysiologischer Erkenntnisse - den Einsatz der Marketinginstrumente als ,geheime Verführer im Hirn des Konsumierenden, und zwar durch Aktivierung des limbischen Systems und durch Ausschüttung von Endorphinen, so zu orchestrieren, dass die Kaufentscheide auf immer subtilere Weise beeinflusst werden können. Dieser Expansionsprozess der Betriebswirtschaftslehre, wissenschaftliche Erkenntnisse aller Disziplinen entsprechend ihrer Bedeutung für die mittelrationale Entwicklung von Instrumenten zur Steigerung des Unternehmenserfolgs $\mathrm{zu}$ verzwecken, schreitet kontinuierlich voran. In diesem paradigmatischen Sinne läge es nahe, auch die CSR-Instrumente einzusetzen, um den Gewinn, z. B. durch das Mehren von margenwirksamen Reputationskapital, zu steigern.

Das Paradigma der Gewinnmaximierung prägt eine praktisch-normative BWL, die freilich ihre Normativität ausschließlich aus diesem Paradigma speist. Das, was praktisch, d.h. in der Unternehmenspraxis, den Unternehmenserfolg steigert, gilt derart als normativ geboten: Dieser hypothetische ,WennDann'-Imperativ gebietet, die notwendigen Mittel für den größtmöglichen Unternehmenserfolg zur Verfügung zu stellen, ohne sie etwa hinsichtlich der anfallenden externen Kosten für Mensch und Umwelt verantwortungsethisch zu reflektieren (vgl. Höffe 1983: 184; Rusche 1993: 67). Auch wird auf eine wissenschaftstheoretische Kritik und Begründung dieses erfolgsrationalen Paradigmas im Forschungsalltag weitgehend verzichtet; es hat sich vielmehr Kraft der Lehrbuchautoritäten fest etabliert und durchzieht das BWL-Studium von den „Fingerübungen“(Kuhn 1991: 61) in den (Pro)-Seminaren und Vorlesungen bis hin zur Bachelor- oder Masterarbeit.

Die Entwicklung von Instrumenten zur erfolgsorientierten Ausgestaltung der Unternehmung führt ,wie bei der Fabrikation, so auch in der Wissenschaft“ (Kuhn 1991: 61) zu einer Ausrüstung mit technischen Hilfsmitteln und zu einem Vorrat wissenschaftlicher Annahmen und Regeln, der für die Gemeinschaft der Fachwissenschaftler*innen zur Selbstverständlichkeit wird. „Solange die normale Forschungsarbeit unter Verwendung des Paradigmas als eines Vorbilds ausgeführt werden kann, brauchen Regeln und Annahmen nicht eigens herausgestellt werden." (Kuhn 1991: 101) Vielmehr nutzt der Wissenschaftler*innen sein ihm vertrautes Rüstzeug, ohne die paradigmatischen Grundlagen kritisch zu reflektieren. 


\section{Gewinnoptimierung als zukunftsverantwortliches Problemlösungsmuster}

Im von Kuhn sogenannten normalen wissenschaftlichen Alltag versucht der Forschende im Rahmen des erlernten Paradigmas Rätsel zu lösen. In diesem Sinne ist die Betriebswirtschaftler*in ein „Rätsellöser, kein Paradigmenprüfer“ (Kuhn 1991: 155). Infolgedessen sucht der betriebswirtschaftlich Forschende immer effizientere Lösungen für das Rätsel, wie durch weitere Outputmaximierung oder Kostenminimierung der Ertrag gesteigert werden kann.

„Solange die von einem Paradigma gelieferten Hilfsmittel sich als fähig erweisen, die von ihm definierten Probleme zu lösen, schreitet die Wissenschaft dann am schnellstens voran und dringt am tiefsten ein, wenn diese Hilfsmittel mit voller Überzeugung gebraucht werden.“" (Kuhn 1991: 89)

Was aber, wenn diese Rätsellösungen innerhalb des Paradigmas zu unlösbaren Problemen führen, weil sie durch das Paradigma selbst verursacht werden? Was tun, wenn das Paradigma in eine Krise gerät und selbst zum unlösbaren Rätsel wird?

Wenn das Paradigma der Gewinnmaximierung in eine Krise gerät, weil menschheitsbedrängende Fragen und Probleme wie z. B. die von erfolgreichen Unternehmen mitverursachten Umweltkatastrophen nicht durch dieses Paradigma verhindert werden können, stellt sich sowohl innerdisziplinär die Frage seiner zukünftigen Relevanz als auch transdisziplinär die Frage seiner ethischen Legitimation. ${ }^{8}$ Neben den psychosozialen Folgekosten von Korruption, Mobbing und Missmanagement belasten insbesondere auch die ökologischen Konsequenzen des gewinnmaximierenden Wirtschaftens das Weltgemeinwohl und lassen die öffentliche, schließlich auch die politische Akzeptanz des Shareholderkapitalismus schwinden. So steht zu befürchten, dass die mittelrationale BWL mit dem paradigmatischen Fetisch der Gewinnmaximierung selbst die Voraussetzungen des erfolgsrationalen Wirtschaftens untergräbt und so - wider Willen - zum Totengräber seiner selbst gerät.

Angesichts dieser Krise wird der öffentliche Ruf nach Übernahme gesellschaftlicher Verantwortung durch die Unternehmensführung immer lauter und manifestiert sich in Dokumenten wie der ISO-Norm 26000. Die weltweit geführte CSR-Diskussion tut ihr Übriges, um die Unzuträglichkeit des Paradigmas der Gewinnmaximierung angesichts der sozial-ökologischen Bedrohung der Menschheit vor Augen zu führen. So wird der paradigmatische Konflikt von Gewinnmaximierung und CSR offensichtlich. Mit dem gewinnmaximierendem Instrumentarium der BWL werden Unternehmen nicht befähigt, ihren

„Ich glaube, daß besonders in Perioden anerkannter Krisen die Wissenschaftler sich der philosophischen Analyse als eines Mittels zur Lösung von Rätseln auf ihrem Gebiet zuzuwenden pflegen." (Kuhn 1991: 101) 
Krisenbeitrag zu leisten. Mittelrationale Instrumente der Gewinnmaximierung versagen bei der zielrationalen Reflexion von Menschenrechten und Umweltschutz und taugen nicht zur ethischen Rechtfertigung von sozialer Verantwortung in der Unternehmensführung. Und selbst für eine Analyse bedarf es anderer Wissenschaften, so der Politikwissenschaften, Soziologie, Medizin, Psychologie, Psychiatrie und Ökologie, um als interdisziplinärer Reparaturbetrieb die Schäden an Mensch und Umwelt zu analysieren und zu behandeln, die von kapitalistischen Unternehmen auf dem Weg zum Gewinnmaximum kollateral (mit-)verursacht werden.

Das Paradigma der Gewinnmaximierung ist in eine Krise geraten; ein Paradigmenwechsel liegt nahe. „Die Bedeutung von Krisen liegt in dem von ihnen gegebenen Hinweis darauf, daß der Zeitpunkt für einen solchen Wechsel gekommen ist.“ (Kuhn 1991: 89) Solange im Rahmen des Paradigmas der Gewinnmaximierung ein scheinbarer Wohlstand für alle erzeugt wurde, schien die Forderung Eugen Schmalenbachs (1873-1955) nach gemeinwirtschaftlicher Wirtschaftlichkeit der gewinnmaximierenden Unternehmen noch erfüllt zu sein. Seitdem aber die Höhe der von den Unternehmen externalisierten sozialen und ökologischen Folgekosten der Globalisierung von der kritischen Öffentlichkeit missbilligt wird, hat eine Aufweichung des Paradigmas eingesetzt.

Ausdruck dieser Paradigmenkrise ist der immer lauter werdende Ruf nach gesellschaftlicher Verantwortungsübernahme der Unternehmen. Allerdings fehlt es diesem Ruf wie zuvor am Beispiel der ISO-Norm 26000 gezeigt, an begrifflicher Präzision, philosophischer Reflexion und kategorialer Unterscheidung der unterschiedlichen Ebenen von ethischer Begründung und moralischer Orientierung sowie an einer Trennung der Sphären von Recht und Moral, von Sein und Sollen. Es fehlt an der Ausarbeitung eines alternativen Paradigmas, welches die betriebswirtschaftliche Ökonomie befähigt, einen Beitrag zur Global Governance zum bonum commune der Weltgesellschaft zu leisten.

Gemeinsam mit Politik- und Rechtswissenschaften, Soziologie, Medizin, Verhaltenswissenschaften und Nationalökonomie sind Philosophie und Betriebswirtschaftslehre gefordert, die CSR-Diskussion vor der Gefahr intradisziplinärer Scheuklappen und interdisziplinärer Sprachlosigkeit zu bewahren. Wie die Philosophie durch Schärfung von Terminologie und Definitionen sowie durch Verbesserung der ethischen Reflexion und der Begründungsmethoden, nicht allein zur Vermeidung von Kategorienfehlern, beitragen kann, habe ich bereits diskutiert. Während mir die Anschlussfähigkeit der Philosophie, insbesondere der Ethik als kommunikativer Unternehmensethik an das CSR- 
Projekt offensichtlich erscheint, bedarf es in der BWL eines Paradigmenwechsels. ${ }^{9}$ Im Folgenden plädiere ich für eine Ablösung des Paradigmas der $\mathrm{Ge}$ winnmaximierung durch das Gewinnoptimum als Problemlösungsmuster, das für ein transdisziplinäres CSR-Projekt anschlussfähig ist.

Im Gewinnoptimum werden die originären Erfolgsinteressen des Unternehmens als produktives soziales System keineswegs ausgeblendet, sondern ebenso berücksichtigt wie die sozialen und ökologischen Zielsetzungen. Abbildung 2 verdeutlicht, wie es in $(\alpha)$ gelingen kann, zunehmende gesellschaftliche Verantwortung zu übernehmen und parallel dazu den quantitativen Periodengewinn zu steigern. Auf dem Scheitelpunkt $\mathrm{G}_{\mathrm{opt}}$ sind ökonomische und gesellschaftliche Verantwortung optimal ausbalanciert. In x nimmt die gesellschaftliche Verantwortung zunehmend ab und geht in y in eine verantwortungslose Profitorientierung über. Die gewinnmaximierenden Unternehmensakteure sind nunmehr bereit, gegen sozialökologische Werte sowie ethische Prinzipien und juristische Normen zu verstoßen, bis sie im Extremfall als mafiöse Organisation über Leichen geht.

Abbildung 2: Gewinnoptimum

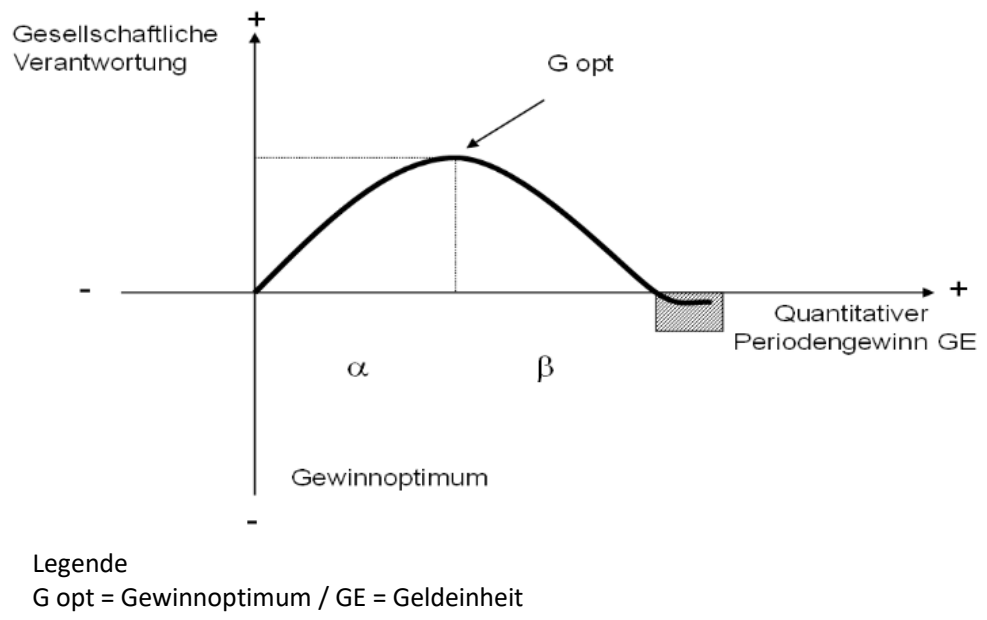

Quelle: eigene Darstellung

9 Nicht nur die fehlende philosophische Methodik ist zu beklagen, sondern auch die ,relativ geringe Bedeutung von CSR in der deutschsprachigen BWL“, die aufgrund der Dominanz ihres Gewinnmaximierungsparadigmas nur erschwerten Zugang zur CSR Diskussion findet (Hansen/Schrader 2005: 379f.). 
Das Streben nach einem solchen Gewinnoptimum setzt die transdisziplinäre und kommunikativ-ethische Einsicht voraus, dass von der Unternehmensführung nicht nur Gewinninteressen der Shareholder, sondern Geltungsansprüche aller Stakeholder zu berücksichtigen sind. Entscheidend für das Gewinnoptimum ist die Orientierung des unternehmerischen Handelns sowohl am originären Erfolgsinteresse des Unternehmens als auch an der Mehrung des Gemeinwohls, dem bonum commune (futurum?) der Gesellschaft. Aus dem ethischen Prinzip einer sozial-ökologisch orientierten Zukunftsverantwortung müssen für die Unternehmensführung konkrete und funktional verantwortbare Sollensnormen entwickelt werden, die den erfolgsrationalen Möglichkeiten entsprechen. In der gesellschaftlichen Diskussion, so zeigt das CSR-Beispiel, ist diese Neuorientierung weiter vorangeschritten als in der Betriebswirtschaftslehre. Auch die Praxis der Unternehmensführung zeigt bereits in bemerkenswerten Einzelfällen die Abkehr von der Gewinnmaximierung und die Orientierung an ökologischen und sozialen Zielsetzungen. ${ }^{10}$

Für die Paradigmendiskussion in der BWL bedarf es jedoch einer weitergehenden Klärung von Begriffen und Konzepten; insbesondere bleibt die Problemlösungsqualität des Paradigmas der Gewinnoptimierung für die offenen und mit dem alten Paradigma unlösbaren betriebswirtschaftlichen Rätsel unserer Zeit aufzuzeigen. Dabei ,wollen wir uns daran erinnern [...:] Wenn ein neuer Paradigma-Anwärter zum ersten Mal vorgeschlagen wird, hat er meistens nur wenige der Probleme, denen er sich gegenübersieht, gelöst, und die meisten dieser Lösungen sind bei weitem noch nicht vollkommen." (Kuhn 1991: 166) Wie kann das neue und noch unvollkommene Problemlösungsmuster der Gewinnoptimierung verbessert werden? Meines Erachtens ist dafür die Überwindung der Aporie der Stakeholdertheorie von entscheidender Bedeutung.

\section{Die Aporie der Stakeholdertheorie}

Kennzeichnend für den CSR-Standard der ISO-Normierungsorganisation ist die ausdrückliche Stakeholder-Orientierung. Ein Unternehmen trägt gemäß ISO-26000 Verantwortung gegenüber allen Stakeholdern. Die Anspruchsträger sind zu identifizieren und bei der Organisationsführung einzubeziehen. So wird die ,Achtung der Interessen der Anspruchsgruppen' zum ethischen Prin-

10 Thomas Kuhn zitiert in diesem Zusammenhang Max Planck: „Eine neue wissenschaftliche Wahrheit pflegt sich nicht in der Weise durchzusetzen, daß ihre Gegner überzeugt werden und sich als belehrt erklären, sondern vielmehr dadurch, daß die Gegner allmählich aussterben und daß die heranwachsende Generation von vornherein mit der Wahrheit vertraut gemacht ist" (zitiert nach Kuhn 1991: 162). 
zip erhoben, ohne zu klären, ob und welche potentiell konfligierenden Ansprüche von unterschiedlichen Interessensträger*innen (über die ,Wahrung ihrer Menschenrechte' hinaus) grundsätzlich achtenswert sind. Nach welchen ethischen Prinzipien soll über die Achtungswürdigkeit dieser Interessen in der Unternehmung entschieden werden? Unter welchen Voraussetzungen werden welche Ansprüche welcher Stakeholder im unternehmerischen Entscheidungsprozess berücksichtigt? Die Beantwortung dieser Kernfrage setzt eine Rechtfertigung ethischer Prinzipien voraus, die ISO-26000 nicht leistet.

Eine solche Anspruchsprüfung ist keine leichte Sache. Denn die Liste möglicher Stakeholder einer Unternehmung ist potentiell unendlich und ihre Interessen sind oftmals konfliktär (vgl. Suchanek 2015: 303). Es erstaunt deshalb nicht, wie vielfältig die Ansätze zur Ermittlung unterschiedlicher Erwartungen von Interessensträger*innen sind. ${ }^{11}$ Neben der Identifikation aller tatsächlichen Anspruchsträger*innen innerhalb und außerhalb einer Unternehmung ist deren Klassifikation für die Unternehmensführung von Bedeutung. Welche Macht besitzen die Stakeholder? Welche Legitimität haben ihre verschiedenartigen Ansprüche? Mit welcher Strategie verfolgen die Stakeholder ihre Interessen? Welchen Aggregationsgrad haben die Interessen? (vgl. Aßländer 2011: 293; Bruton 2011: 167; Mitchell/Agle/Wood, 1997: 874f.) Je nachdem, ob ein Stakeholder seine mehr oder weniger legitimen Interessen machtvoll oder zurückhaltend als strategischer Gegenspieler oder kommunikativer Dialogpartner vorbringt und ob es sich um individuelle, institutionelle oder gesellschaftliche Interessen von Stadt, Land, Bund oder gar um solche von internationaler Relevanz handelt, ergibt sich für mich als Akteur meines Unternehmens eine andere Ausgangssituation für die Stakeholderdialoge (vgl. Wieland 2007: 14f.; Wallacher 2006: 98). Im Perspektiventausch sollte mir als Unternehmensagent bewusst sein, dass auch meine Institution von Dritten als Stakeholder wahrgenommen und bzgl. mehr oder weniger systematischer Klassifikationskriterien eingeschätzt wird.

Auf Grundlage dieser deskriptiven Perspektive der ggf. wechselseitigen Ermittlung von Stakeholdern und ihrer Attribute geht es zunächst darum, eine differenzierte Übersicht der unternehmensrelevanten Umwelt zu gewinnen. Eine übersichtliche Stakeholderlandkarte des Beziehungsgeflechts einer Unternehmung kann für den Unternehmenserfolg genutzt werden und der erfolgsrationalen Ausgestaltung der Austauschbeziehungen dienen. Werden die Anspruchsträger ausschließlich zur Maximierung des Shareholdervalues instrumentalisiert oder wird ein fairer, an universalen ethischen Prinzipien ausgerichteter Interessensausgleich aller Stakeholder angestrebt? Ob und in welchem Umfang die Unternehmensführung das Netzwerk von Beziehungen für den Unternehmenserfolg ausschließlich instrumentalisiert, und/oder darüber

11 Die Liste der sogenannten Stakeholder umfasst, any group or individual that can affect or is affected by the achievement of a corporation's purpose" (Freeman 2004: 228). vgl. auch Freeman/Evan 1990: 337f.; Bruton 2011: 164 
hinaus normativ-ethische Aspekte berücksichtigt, hängt m. E. wesentlich von der Individualmoral der handelnden Unternehmensagenten ab sowie von den spezifischen Ausprägungen einer mehr oder weniger ethisch aufgeklärten Unternehmenskultur. Der Stakeholderansatz selbst weist keinen methodischen Weg, wie ethische Kriterien zur Prüfung der Ansprüche von ggf. konfligierenden Interessensträger*innen innerhalb und außerhalb der Unternehmung begründet und moralrelevant ins Spiel der Unternehmensführung gebracht werden könnte. Eben darin liegt praktische Aporie der Stakeholdertheorie (vgl. Bruton 2011: 176; Donaldson/Preston 1995: 65).

\section{Diskursethische Fundierung der Stakeholder-Dialoge}

Angesichts der moralphilosophischen Blindheit der Stakeholdertheorie postuliere ich die Anschlussmöglichkeit der Diskursethik an die Stakeholdertheorie (vgl. Wieland 2014: 73f.; Phillips/Freeman/Wicks 2003: 479f.). Schließlich kann nur im Diskurs mit den Interessensträger*innen der Sinn ihrer Ansprüche erschlossen und deren Legitimität geprüft werden. ${ }^{12}$

$\mathrm{Da}$ in Stakeholder-Dialogen die Geltungsansprüche der Anspruchsträger*innen potentiell divergieren, bedarf es einer diskursethischen Geltungsreflexion, um die Zustimmungswürdigkeit der unterschiedlichen Interessen klären zu können. ${ }^{13}$ Letztlich hängt hier alles daran, dass sowohl der Unternehmensagent als auch die Unternehmenskultur gegenüber den Stakeholderansprüchen diskursfähig sind: Können sie, unabhängig von der Durchsetzungskraft der Stakeholder, deren Interessen auf ihre moralische Legitimität hin prüfen?

Da die Unternehmung eine erfolgsrationale Organisation mit der Tendenz ist, zeitraubende Diskurse zu vermeiden, gilt es, ebendort solche zu etablieren. Hier mit dem Ziel, die Ansprüche der Stakeholder zu prüfen; und zwar unter der Frage, ob sie - hinsichtlich aller zu erwartenden Konsequenzen - für die Unternehmensakteure zumutbar und angesichts eingegangener Verpflichtungen gegenüber Dritten verantwortbar und mithin zustimmungswürdig sind.

Die argumentative Prüfung der Konsenswürdigkeit aller von Stakeholdern vorgetragenen Geltungsansprüche bringt den moral-point-of-view ins Spiel der Stakeholdertheorie und -praxis (vgl. Göbel 2005: 103f.). Diesen Diskurs über

12 Nils Goldschmidt und Karl Homann postulieren in diesem Zusammenhang eine ,Diskursverantwortung' der Unternehmen (vgl. Goldschmidt/ Homann 2011: 35 f.).

13 „Um die Frage nach der Legitimität der jeweiligen Stakeholder-Interessen zu klären, verweisen Thomas Maak, Peter Ulrich, Albert Löhr und andere Autoren daher auf die Grundlagen der so genannten Diskursethik, die ein Verfahren für die Verständigung über die Geltung und Anwendung moralischer Normen definiert“ (Aßländer 2011: 296). vgl. Maak/Ulrich 2007: 181f.; Ulrich 1997: 438 ff. 
die Interessen der Anspruchsträger*innen aus den unterschiedlichsten Rationalitäts- und Interessensperspektiven zu ermöglichen und für alle Stakeholder trotz (zeit-)aufwändiger Abstimmungsprozesse offen zu halten und die Diskursvoraussetzungen trotz potentiell divergierender Interessen und strategischer Antagonismen stetig zu verbessern, ist das diskursethische Fundament der Stakeholderdialoge: Ermögliche den Diskurs - Argumentiere sinnvoll und sei ein glaubwürdiger Diskurspartner - Strebe nach argumentativen Konsens und verbessere die Dialogchancen. (vgl. Westermann-Behaylo/van Buren /Bermann 2016: 542)

Der Teilimperativ ,Ermögliche den Diskurs und verbessere die Dialogchancen' fordert sowohl eine persönliche Verantwortung für mein Dialogverhalten, als auch meine Mitverantwortung für die Chancen im Unternehmen und in der (Welt-)Öffentlichkeit Stakeholderdialoge zu führen. Außerdem ist unsere Mitverantwortung für die Shareholderdialoge gefordert: Für die gesellschaftlichen Kommunikationsbedingungen, zu denen sowohl die Rechtsverhältnisse als auch die Ursachen öffentlicher Diskurse gehören. Je nach Umfeld von beteiligten Unternehmen und betroffenen Gesellschaften sind in den Shareholderdialogen unterschiedliche Spannungen von ökonomischer Effizienz und sozial-ökologischen Imperativen diskursiv aufzuarbeiten, wie z. B.: Fair Trade, nachhaltiger Anbau, ressourcenschonende Produktion und Logistik, Transparenz und Kontrolle der Zulieferketten, Abbau von Korruption, menschenwürdige Produktionsverhältnisse usw.

Die Diskussionen mit den Stakeholdern zielen auf vollendete Gegenseitigkeit. Vollendete Reziprozität ist das entscheidende Moralkriterium für Stakeholderdialoge. Als vollendete Dialoggegenseitigkeit gedeutet, eröffne sie angesichts der faktisch gegebenen und aus Effizienzgründen oftmals nötigen kommunikativen Restriktionen größtmögliche gegenseitige Dialogchancen. Wer Dialogchancen eröffnet, ermöglicht den Diskurs und hält ihn offen für neue, zeitlich später hinzukommende Shareholder und ihre inhaltlichen Beiträge.

Die eben genannten Teilimperative explizieren zugleich das primordiale diskursethische Prinzip ,Argumentiere sinnvoll und sei ein glaubwürdiger Dialogpartner', welches implizit dazu verpflichtet, in den Shareholderdialogen nach einem Konsens der Argumente zu streben und die Dialogfähigkeit zu verbessern.

Dabei will ich die Erwartungen an einer Funktionalisierung dieser ethischen Regeln nicht zu hoch ansetzen. Es geht mir schlicht und einfach darum, die Aporie der Stakeholdertheorie durch Dialoge über alle Aspekte und relevanten Folgen einer zwischen Stakeholdern verhandelten Sachfrage zu fördern.

Sozial-ökologische Verantwortung kann eine Unternehmung nur im Blick auf konkrete Stakeholder übernehmen. Um diese Verantwortung inhaltlich zu konkretisieren bedarf es der ethisch qualifizierten Dialoge mit möglichst allen 
Beteiligten und Betroffenen. Ziel ist ein gerechter Interessensausgleich im Diskurs, der die Unternehmensführung in die Lage versetzt, die ökonomische und gesellschaftliche Verantwortung auszubalancieren. Zusammengefasst: Das CSR-Versprechen einer Unternehmung erfüllt sich im Gewinnoptimum, das mittels diskursethisch fundierter Shareholderdialoge erreicht werden kann.

\section{Literatur}

Abel, B. (1979): Denken in theoretischen Modellen als Leitidee der Wirtschaftswissenschaften. In: Raffée, H./Abel, B. (Hrsg.): Wissenschaftstheoretische Grundlagen der Wirtschaftswissenschaften. München: Vahlen, S. 215-234.

Apel, K.-O. (1988): Diskurs und Verantwortung. Frankfurt/M: Suhrkamp.

Aßländer, M. S. (2009): Die Soziale Verantwortung der Unternehmen. In: Aßländer, M. S./Senge, K. (Hrsg.): Corporate Social Responsibility im Einzelhandel. Ethik und Ökonomie. Band 8. Marburg: Metropolis-Verlag. S. 25-54.

Aßländer, M. S. (2011): Grundlagen der Wirtschafts- und Unternehmensethik. Marburg: Metropolis-Verlag.

Aßländer, M. S./Brink, A. (2008): Begründung korporativer Verantwortung: Normenkonkretion als Prozess. In: Scherer, A. G./Patzer, M. (Hrsg.): Betriebswirtschaftslehre und Unternehmensethik. Wiesbaden: Gabler, S. 103-124.

Bruton, J. (2011): Unternehmensstrategie und Verantwortung. Berlin: Erich Schmidt Verlag.

Carroll, A. (1999): Corporate Social Responsibility. In: Business \& Society, Band 38, S. 268-295.

Carroll, A. (2009): A History of Corporate Social Responsibility. In: Crane, A. u. a. (Hrsg.): The Oxford Handbook of Corporate Social Responsibility. New York: Oxford University Press, S. 19-46.

Deutsches Institut für Normierung e.V. (2011): DIN ISO 26000. Leitfaden zur gesellschaftlichen Verantwortung (ISO 26000:2010). Ausgabe 2011-01. Berlin

Donaldson T./Preston, L. E. (1995): The Stakeholder Theory of the Corporation. In: AMR 20, 1, S. 65-91.

Freeman, R. E. (2004): The Stakeholder Approach Revisited. In: zfwu 5, 3, S. 228.

Freeman, R. E./Evan, W. M. (1990): Corporate governance: A stakeholder interpretation. In: The Journal of Behavioural Economics 19, 4, S. 337-359.

Göbel, E. (2005): Stakeholder-Management. In: Brink, A./Tiberius V. A. (Hrsg.): Ethisches Management. Bern u. a.: Haupt, S. 87-129.

Goldschmidt, N./Homann, K. (2011): Die gesellschaftliche Verantwortung der Unternehmen. München: Roman-Herzog Institut.

Hansen, U./Schrader, U. (2005): Corporate Social Responsibility als aktuelles Thema der Betriebswirtschaftslehre. In: DBW 65, 4, S. 373-395.

Hardtke, A. (2010): Das CSR-Universum. In: Hardtke, A./Kleinfeld, A. (Hrsg.): Gesellschaftliche Verantwortung von Unternehmen. Wiesbaden: Gabler Springer Fachmedien, S. 13-70.

Hiß, St. (2006): Warum übernehmen Unternehmen gesellschaftliche Verantwortung? Frankfurt/M.: Campus Forschung. 
Höffe, O. (1983): Immanuel Kant. München: C.H. Beck.

Jones, T. M. (1995): Instrumental Stakeholder Theory. In: AMR 20, 2, S. 404-437.

Küpper, H.-U. (2011): Unternehmensethik. 2. Aufl., Stuttgart: Schäffer-Poeschel.

Kuhn, T. S. (1974): Logik der Forschung oder Psychologie der wissenschaftlichen Arbeit. In: Lakatos, I./Musgrave, A. (Hrsg.): Kritik und Erkenntnisfortschritt. Braunschweig: Vieweg, S. 1-24.

Kuhn, T. S. (1991): Die Struktur wissenschaftlicher Revolutionen. 11. Aufl.. Frankfurt/M.: Springer Link.

Maak, T./Ulrich, P. (2007): Integre Unternehmensführung. Ethisches Orientierungswissen für die Wirtschaftspraxis. Stuttgart: Poeschel.

Mitchell, R. K./Agle, P. R./Wood, D. J. (1997): Toward a theory of stakeholder identification and salience. In: AMR 22, 4, S. 853-886.

Phillips, R./Freeman, R. E./Wicks, A. C. (2003): What Stakeholder Theory Is Not. In: BEQ 13, 4, S. 479-502.

Popper, K. R. (1974): Die Normalwissenschaft und ihre Gefahren. In: Lakatos, I./ Musgrave, A. (Hrsg.): Kritik und Erkenntnisfortschritt. Braunschweig: Wies, S. 51-57.

Rusche, T. (2009): Das ABC des CSR - Chancen und Nebenwirkungen einer neuen Moralstrategie. In: Aßländer, M. S./Senge, K. (Hrsg.): Corporate Social Responsibility im Einzelhandel. Ethik und Ökonomie, Band 8. Marburg: Metropolis-Verlag, S. 55-77.

Rusche, T. (1993): Philosophische versus ökonomische Imperative einer Unternehmensethik. EWD, Band. 2. 3. Aufl. Münster, Hamburg.

Suchanek, A. (2015): Unternehmensethik. Tübingen: Mohr Siebeck.

Toulmin, S. (1974): Ist die Unterscheidung zwischen Normalwissenschaft und revolutionärer Wissenschaft stichhaltig? In: Lakatos, I./Musgrave, A. (Hrsg.): Kritik und Erkenntnisfortschritt. Braunschweig: Vieweg, S. 39-47.

Ulrich, P. (1977): Die Großunternehmung als quasi-öffentliche Institution. Stuttgart: Schäffer-Poeschel.

Ulrich, P. (1997): Integrative Wirtschaftsethik. Grundlagen einer lebensdienlichen Ökonomie. Bern, Stuttgart: Gabler Edition Wissenschaft Verlag.

Wallacher, J. (2006): Unternehmensethik im Kontext von Global Governance. In: Wallacher, J./Reder, M. u. a. (Hrsg.): Unternehmensethik im Spannungsfeld der Kulturen und Religionen. Stuttgart: Kohlhammer, S. 85-107.

Westermann-Behaylo, M. K./van Buren, H.J./Bermann, S. L. (2016): Stakeholder capability enhancement as a path to promote human dignity and cooperative advantage. Business Ethics Quarterly 26, 4, S. 529-555.

Wieland, J. (2007): Idealistische, ideale und reale Diskurse. In: Wieland, J. (Hrsg.): Governanceethik und Diskursethik - ein zwangloser Diskurs. Marburg: Metropolis-Verlag, S. 13-59.

Wieland, J. (2014): Governance Ethics: Global value creation, economic organization and normativity. Cham, Heidelberg u. a.: Springer Cham. 


\section{Chancen und Herausforderungen von CSR in der Sozialwirtschaft und Sozialen Organisationen}

Roundtable beim CSR-Forum „Corporate Social Responsibility: Nutzen verantwortlichen und nachhaltigen Handelns für soziale Organisationen und Gesellschaft" am 29. Mai 2018 in Fulda

Der Roundtable befasste sich mit den Fragen:

- Wer sind die Beteiligten am Roundtable (Vorstellung der Teilnehmer*innen)?

- Wie kann man als Textilunternehmer ein gutes Beispiel in Sachen CSR sein?

- Sozialwirtschaftliche Organisationen als gesellschaftlich verantwortlich handelnde Unternehmen per se, oder hinken sie den profitorientierten Unternehmen in punkto CSR hinter?

- Was ist CSR? Befolgung von rechtlichen Vorgaben oder moralische Auseinandersetzung mit der Daseinsberechtigung und Leitwerten des Unternehmens?

- Wie können sozial- und profitwirtschaftliche Unternehmen angesichts hoher Regulierung und Anforderungen von Seiten des Staates an diese (compliance) noch Zeit für CSR finden?

- CSR als Antwort auf Staatsversagen?

- Wie kann CSR praktisch umgesetzt werden (auch konkrete Beispielprojekte)?

Teilnehmer*innen des Roundtable waren:

- Thomas Domnick, Stv Vorstandsvorsitzender der Liga der Freien Wohlfahrtspflege in Hessen e. V.

- Dr. Dr. Thomas Rusche, SØR Rusche GmbH

- Günter Sandfort, M.Sc., Stv. Diözesan-Caritasdirektor, Osnabrück

- Prof. Dr. Markus Scholz, FHWien der WKW

- Annette Wippermann, Der PARITÄTISCHE Wohlfahrtsverband, LV Hessen e. V.

Moderation: Hermann Diel, Hessischer Rundfunk 
Diel: Herzlich Willkommen zu diesem Roundtable. Mein Name ist Hermann Diel. Ich arbeite hauptberuflich beim hessischen Rundfunk. Wir werden in der kommenden Stunde versuchen uns dem Thema CSR zu nähern. Ich habe keine Ahnung von dem Thema, dafür aber die Menschen um mich herum umso mehr. Zwei der Experten [Anm.: Prof. Dr. Markus Scholz; Dr. Dr. Thomas Rusche] haben wir schon kennengelernt und ich bitte die anderen drei, sich kurz vorzustellen. Wer sind Sie und was ist Ihr Bezugspunkt zum Thema CSR? Fangen wir mit der Dame an.

Wippermann: Mein Name ist Anette Wippermann. Ich bin beim PARITÄTISCHEN Wohlfahrtsverband Landesverband Hessen Referentin im Fachreferat Arbeitsmarktpolitik und im Fachreferat Grundsatzfragen. Vielleicht ganz kurz zum PARITÄTISCHEN, da wird jetzt viel zur Caritas und Diakonie gehört haben. Der PARITÄTISCHE ist auch ein großer Wohlfahrtsverband mit 800 sozialen Organisationen aus allen Bereichen der Sozialen Arbeit. Hier in Hessen haben wir die Landesgeschäftsstelle in Frankfurt. Dort bin ich Grundsatzreferentin. Unser Bezug zu CSR ist, dass natürlich auch immer wieder Organisationen aus unseren Reihen, die bei uns Mitglied sind, auf uns zukommen und mit uns das Gespräch suchen, welche Maßnahmen sie umsetzen sollen, was wir Ihnen raten, von was wir Ihnen abraten. Wir sind nicht ganz unkritisch gegenüber CSR und vielleicht kommen wir nachher nochmal drauf zurück, in dem ich ein paar Punkte, die wir sehr kritisch sehen, nochmal erwähne.

Sandfort: Mein Name ist Günter Sandfort. Ich bin Mitglied im Vorstand des Caritasverbandes für das Bistum Osnabrück und dort zuständig für die Querschnittsaufgaben in der Organisation. Ich leite die Abteilung Grundfragen Europa und Engagementförderung und in diesen Zuständigkeitsbereich fällt auch das Thema CSR. Als Organisation beschäftigen wir uns schon mehrere Jahre mit CSR. Das Ganze hat seinen Ursprung darin, dass wir uns an einem Projekt der deutschen Bundesumweltstiftung mit dem Titel „Zukunft einkaufen“ beteiligt haben. Da ging es um das Thema nachhaltige Beschaffung. Das war der ausschlaggebende Punkt, um zu sagen: wir müssen unsere Aktivitäten jetzt nicht nur auf Fragen von Beschaffung und Umweltschutz beziehen, sondern CSR geht viel weiter. Dann haben wir mit Unterstützung des CSR-Kompetenzzentrums im Deutschen Caritasverbandes eine eigene Strategie entwickelt.

Domnick: Mein Name ist Thomas Domnick. Im Hauptberuf bin ich Diözesancaritasdirektor im Bistum Mainz. Heute bin ich aber in der Rolle des stellvertretenden Vorsitzenden der Liga der freien Wohlfahrtspflege hier. Frau Wippermann sagte schon, dazu gehören der PARITÄTISCHE, die Caritas, die Diakonie, aber auch die Arbeiterwohlfahrt, das Deutsche Rote Kreuz und der jüdische Gemeindebund. Das heißt im Grunde ist das, was vorhin [von Herrn Scholz] ausgeführt wurde, die Collective Action, genau das was wir als Liga 
machen - ein Zusammenschluss von Wohlfahrtsverbänden. Dort schauen wir, wie wir Standards entwickeln können, die dann auch zielführend sind.

Diel: Vielen herzlichen Dank. Sie kennen jetzt die Personen, die hier vorne stehen. Die Spielidee ist, dass ich zunächst ein paar vorbereitete Fragen stellen werde, um in das Thema eintauchen, aber am Ende wollen wir ins Gespräch kommen.

Herr Rusche, ich habe mir für Sie, eine Einstiegsfrage aufgeschrieben, die sie als Provokation verstehen könnten. Deshalb schiebe ich mal vorweg, das ist wirklich eine Frage und nichts, um Sie zu ärgern. Sie sind ja hier, weil Sie das 'gute Beispiel' sind. Sie sind der Vertreter des Profitunternehmens, aber einer, der Gutes tut. Sie haben Ihre Biografie sehr klein gehalten. Ich habe Sie mal gegoogelt: Sie waren am Jesuitengymnasium. Sie haben Theologie studiert. Sie haben ein eigenes Forschungsprojekt „Ethik und Wirtschaft“ durchgeführt. Sie sind Ritter vom Heiligen Grab. Sie sind beim Kolpingwerk. Sie sind ausgezeichnet worden für das Besoldungssystem ihrer Mitarbeiter. Sie sind also 'der Gute' in unserer Runde hier und dann kommen Sie aus der Textilbranche. Wenn wir jetzt unter die grundsätzlichen Branchen, die wir auf dieser Welt haben, einen Strich machen würden, dann ist, glaube ich, auf der schlimmen Seite (zumindest vom Klischee her) das Textilwesen. Schlechte Löhne, schlimme Arbeitsbedingungen, wir haben vorhin ein bisschen was gehört. Wie kann man als Textilunternehmer ein gutes Beispiel sein?

Rusche: Ich glaube, dass Sie den sozialen Nutzen der Kleidung völlig unterschätzen. Sie, obwohl Sie Moderator sind und mit Worten in wunderbarerweise Ihre Gedanken ausdrücken können, sprechen mir Ihrer Kleidung schon bevor Sie Ihren Mund aufmachen. Mode hat eine unglaubliche Signal- und Symbolfunktion. Wir alle sollten Mode als Kommunikationsinstrument nicht unterschätzen. Wenn Sie über Kommunikationsmedien nachdenken, kann ich allerdings verstehen, dass Sie heute eher an Smartphones denken, als an gut geschnittene Sakkos. Wenn wir jetzt aber mal die Ökobilanz eines Smartphones vergleichen würden mit der Ökobilanz eines gut sitzenden Sakkos, dann habe ich als Modeunternehmer, glaube ich, einen ganz großen Vorteil. Punkt.

Diel: Herr Scholz hat uns ja die Bilder von Einsturz des Rana Plaza in Bangladesch und was Collective Action in dem Fall gebracht hat gezeigt. Die Löhne sind gestiegen und die Arbeitsbedingungen sind besser geworden. Jetzt habe ich aber vor wenigen Tagen in einem regionalen Blatt gelesen, dass Bangladesch inzwischen der Textilindustrie zu teuer geworden ist. Die ist jetzt weitergewandert nach Äthiopien. Sie haben ja selbst von der Doppelbödigkeit der guten Taten gesprochen. Wie ordnen wir dann so etwas ein?

Rusche: Ich denke wir müssen das ganz grundsätzlich anschauen. In meiner Kleinstadt Oelde gab es nach dem Krieg 50 Schneider. So ähnlich wie man die 
Dinge lokal vom Bauernhof besorgte, wenn man nicht selbst im Garten anbaute, wurde auch um die Ecke die Kleidung produziert. Das ist jetzt nach 50 Jahren mit größtem Transportlogistikaufwand und mit größter Ressourcenverschwendung ganz anders organisiert. So wie Ihnen keiner wirklich sagen kann, was alles in einem Smartphone wo wann wie produziert und hin und her geflogen wurde, so ist auch kaum einer in der Lage, das sage ich in aller Offenheit, bei meinen 50.000 Produkten, die ich im Sortiment habe, deutlich zu machen, was wann wo unter welchen Umständen produziert wurde. Ich sage auch Zertifizierung hin oder her, sie kriegen für einen Euro jedes Ökosiegel, jeden Fairtrade-Beleg, aber was dann wirklich substanziell dahintersteckt, weiß wirklich keiner. Das heißt, auch da müssen wir beim Konsumenten beginnen. Wenn dieser in Umfragen befragt wird, ob er bereit sei für ein ökologisches, fairgetradetes Produkt, für das Ei vom Bauernhof nebenan 20 Cent mehr zu zahlen, in größter Weise diese Bereitschaft hat, aber vor dem Supermarktregal, wie wir alle wissen, sehr oft einknickt. Auch da müssen wir uns alle dieser Doppelbödigkeit bewusstwerden und entscheiden, wie wir damit umzugehen haben. Ich glaube der Pendelschlag, der extreme Pendelschlag, oft beschrieben am Beispiel der Nordseekrabbe, die nach Marokko geflogen wird, um sie dort auszupulen und zwei Tage später wird sie dann wieder am Nordseestrand verkauft. Dieser extreme Pendelschlag ist erreicht und muss und wird in eine andere Richtung gehen.

Diel: Vielen Dank. Ich glaube, wir haben zwei Grundprinzipien erkannt. Das eine kennen wir ja vom Ei, wenn wir vor dem Supermarkt die Umfrage machen, welche Eier möchten Sie kaufen, dann sind das alles die Eier von glücklichen Hühnern, die frei leben und dann gehen dieselben Leute, die gerade noch ja angekreuzt haben, rein und kaufen das billigste, was sie finden - das ist, glaube ich, auch nicht neu.

Das zweite sind die Mechanismen des Marktes in der Textilindustrie. Ich glaube, in Fulda muss man das nicht erklären. Noch vor ein paar Jahrzenten war der Mehler der größte Arbeitgeber hier. Da wurde die Stadt bunt, wenn die Näherinnen zurückkamen und heute ist davon fast nichts mehr übriggeblieben. Die einzige Oberbekleidung, die wir hier noch machen, wenn ich es richtig sehe, sind kugelsichere Westen mit, glaube ich, 30 Mitarbeitern. Wir haben das hier alles vor Ort erlebt - die Mechanismen sind bekannt.

Aber wir wollen ja eigentlich über CSR sprechen. Frau Wippermann, wir haben heute das Zitat von Patrick Hofmacher, Mitglied der Geschäftsführung der Malteserwerke Köln, schon mehrfach gehört und ich habe es mir auch aufgeschrieben: „Nur weil wir als katholischer Dienstleister in den Bereichen der Jugendhilfe und Migration Gutes tun, bedeutet das noch nicht, dass wir automatisch auch in anderen Bereichen der Unternehmensführung gut sind." Hin- 
ken soziale Unternehmen, gerade in diesen Bereichen kommerziellen oder Profitunternehmen hinterher? Der Schuster hat ja oft gerne die schlechtesten Schuhe.

Wippermann: Also hinterher hinken würde ich es nicht nennen. Das kann man, glaube ich, nicht sagen. Ich glaube, die Sozialwirtschaft hat natürlich ihre Ziele per se in ihrem Leitbild benannt und muss sich daher die Frage stellen, inwieweit setzt sie die in ihren eigenen Unternehmungen um. Da würde ich sagen, hat sie oft härtere Bedingungen als die freie Wirtschaft. Das klingt jetzt vielleicht ungewöhnlich, aber das ist so. Ein Großteil der Sozialwirtschaft rechnet härter als in der freien Wirtschaft und das wirkt sich natürlich auf die gesamten Arbeitsbedingungen aus. Da wird mit befristeten Arbeitsverträgen gearbeitet, da wird mit Projektstellen gearbeitet, da wird das Geld, was nur für Projekte kommt, auch nur für Projekte ausgegeben etc. Das heißt, die Sozialwirtschaft unterliegt einem harten Druck und entsprechend wirkt sich das auf die internen Strukturen aus und entsprechend schwierig ist es oft, interne soziale Standards zu halten. Trotzdem würde ich nicht sagen, dass die Sozialwirtschaft schlechter ist als die freie Wirtschaft. Sie unterliegt einem harten Wettbewerbsdruck, den auch die freie Wirtschaft hat, aber sie ist mehr Rechenschaft schuldig als die freie Wirtschaft. Also ich denke schon, die Sozialwirtschaft muss sich mehr Kritik gefallen lassen, dazu was für Arbeitsverhältnisse sie hat, etc. und sich dem mehr stellen. Sie (Herr Sandfort) möchten das ergänzen?

Sandfort: Ich würde Ihnen an dieser Stelle umfänglich zustimmen wollen. Die Bedingungen sind einerseits sehr schwierig. Auf der anderen Seite haben wir aber auch - und da sind wir dann beim Thema CSR - eine Fürsorgepflicht gegenüber unseren Mitarbeitern und Mitarbeiterinnen und stehen in einer ganz besonderen Verantwortung ihnen gegenüber. Deswegen haben wir in unserer Organisation Führungsleitlinien entwickelt. Wir haben darüber hinaus überlegt, wie können wir das, was uns wichtig ist, für unsere Mitarbeiter ins Bild bringen. Wie können wir ihnen Leistungen ermöglichen, die sie bei uns bekommen und an einer anderen Stelle nicht erhalten und damit letztendlich zu einem Arbeitgeber werden, bei dem die Mitarbeiterinnen und Mitarbeiter gerne arbeiten. Das ist, denke ich, ein Kernthema von CSR.

Ich würde aber ganz gern nochmal kurz auf Herrn Dr. Rusche eingehen, Stichwort Textil. Auch da gibt es ja durchaus Möglichkeiten des Gegensteuerns. Wir beginnen jetzt eine Kooperation mit einem Startup in Köln, die Firma heißt Kaya und Kato und stellt Dienstkleidung her, die nachhaltig produziert worden ist. Sie sprachen vorhin von Vertrauen und Glaubwürdigkeit. Das ist sicherlich ein ganz entscheidender Punkt an der Stelle. Ich weiß um das Problem mit den Zertifikaten und wie wenig Glaubwürdigkeit solche Zertifikate haben. Anders ist es, wenn es Personen sind, die für eine Sache einstehen. In 
dem Fall (Firma Kaya und Kato) kann ich das so überzeugt sagen, weil der Unternehmensgründer zehn Jahre für die UNESCO unterwegs war. Die Ware, die Baumwolle, wird unter anderem in Uganda produziert und da bin ich sicher, dass die Kriterien eingehalten werden, die uns an dieser Stelle wichtig sind.

Diel: Vielen Dank. Herr Domnick, Sozialverbände sind anscheinend doppelt beteiligt und doppelt betroffen. Sie sind einerseits oft Partner von Unternehmen, die mit ihnen ein Projekt im Bereich CSR umsetzen, aber Sie sind umgekehrt selbst der Unternehmer. Macht es das dann auch doppelt schwierig?

Domnick: Ja, mit Sicherheit. Frau Wippermann hat das angesprochen. Wir haben letztes Jahr eine Studie gemacht, eine Sozialwirtschaftsstudie für Hessen als Liga der Wohlfahrtspflege. Etwa 113.000 Menschen arbeiten in den Wohlfahrtsverbänden in etwa 7.500 Einrichtungen. Von den Zahlen her wird deutlich, dass wir da eine doppelte Verpflichtung haben, denn auf der einen Seite sind wir für Menschen da, das ist unser Leitbild. Wenn ich in die Pflege schaue, sind wir in erster Linie für die Menschen da, die sich uns anvertrauen in unseren Altenheimen, in ihren Wohnungen über die ambulante Pflege und über die Sozialstation und gleichzeitig müssen wir natürlich schauen, dass die Pflegerinnen und Pfleger, die Altenpflegerinnen auch ordentlich entlohnt werden und ordentliche Rahmenbedingungen haben. Da merken wir schon, dass wir immer wieder an Grenzen kommen. Der Fachkräftemangel wurde angesprochen. Das heißt natürlich für eine Altenpflegerin heute, wenn sie ihren Dienst getan hat und frei hat, wird sie unter Umständen angerufen, weil jemand krank ist, weil jemand Urlaub hat oder kurzfristig ausfällt und dann muss sie einen weiteren Dienst machen. Das sind natürlich keine optimalen Arbeitsbedingungen. Von daher sind wir da als Wohlfahrtsverbände immer auf der Rasierklinge zu schauen, wie wir einerseits gute Arbeitsbedingungen ermöglichen können, und andererseits aber auch den Dienst an den Menschen leisten. Das ist natürliche eine Schwierigkeit, wo man immer wieder nachsteuern muss.

Diel: Herr Scholz, Sie würde ich gern etwas fragen, dass ein bisschen davon weggeht. Ich versetze mich jetzt mal in die folgende Lage: da ist jetzt jemand, der hat eine tolle Idee, oder kann toll Schuhe machen oder verkauft irgendein Produkt. Jetzt macht er sich selbstständig, wächst ein bisschen, hat dann sieben, acht Mitarbeiter. Dann hat er die Steuer und die Sozialversicherung an der Backe, dann muss er sich mit der Inklusion auseinandersetzen, dann muss er schauen, ob der Frauenanteil in seinem Unternehmen ordentlich ist, dann muss er alles dokumentieren und muss gleichzeitig schauen, dass er alle Daten gelöscht hat. Dann muss er sich um den Umweltschutz kümmern und das alles auch noch dokumentieren und zertifizieren. Wo bitte hat der dann noch Zeit und Nerven sich um CSR zu kümmern? 
Scholz: Hier sprechen Sie ja fast wie jemand von einem Arbeitgeberverband.

Diel: Den haben wir ja nicht hier.

Scholz: Schade. Das Thema, das Sie ansprechen, kann vielleicht unter der Überschrift „Compliance“ zusammengefasst werden. Von Seiten des Staates werden hohe Anforderungen an Unternehmen gestellt. Sie haben das bereits angesprochen, da ist die Steuer, der Datenschutz, Transparenz, die Einhaltung von zahlreichen Standards und das ist mitunter erdrückend für Unternehmen. Ich lebe in Wien und der Präsident der österreichischen Industriellenvereinigung, Georg Kapsch, sagt in vielen Interviews, dass es zu viele ComplianceVorschriften gibt und sich die Unternehmen überhaupt nicht mehr um ihre Kunden und Kundinnen, um Innovationen oder um Mitarbeiterausbildung kümmern können, weil sie sich so mit Compliance befassen müssen. Ein Monstrum, mit dem wir uns gerade herumplagen, ist die Datenschutzverordnung der EU. Hintergrund ist der Umgang der Unternehmen mit nutzerbezogenen Daten zur Verwendung für Werbezwecke mit Nichtkonsens, also mit Nichteinverständnis der Nutzerinnen und Nutzer. Und das führt dann zu den entsprechenden Richtlinien und wahrscheinlich wird noch mehr Compliance auf uns zukommen. Es sei denn, Unternehmen und andere Organisationen fangen jetzt an, „das Richtige“ zu tun - was auch immer das genau ist. Denn die Megatrends geben Anlass dazu, beispielsweise Digitalisierung, der Umgang mit neuen Geschäftsbedingungen oder neuen Geschäftsmodellen. Das Unternehmen Uber ist kurzfristig verschwunden - vom Pariser Markt, vom Wiener Markt, vom Berliner Markt. Aus welchem Grund? Weil es eine starke Gegenbewegung in der Gesellschaft gibt: Einerseits ist Uber zwar ein toller und praktischer Service, den wir gerne alle nutzen, da er nämlich günstiger und praktischer als Taxifahren ist. Auf der anderen Seite werden so viele Standards, die gesellschaftlich anerkannt aber noch nicht formalisiert sind, nicht eingehalten. Die Reaktion des Gesetzgebers war jetzt ein Verbot von Uber. Das heißt, Unternehmen, die mit diesen neuen Technologien und Geschäftsmodellen arbeiten müssen von jetzt an eigeninitiativ diese Themen adressieren und sagen: „Wir tun von jetzt an das Richtige“ - was auch immer das genau ist. Das muss dann mit den Stakeholdern ausgehandelt werden.

Diel: Die Frage gebe ich dann gern an Herrn Rusche weiter. Wenn wir Compliance sagen ist das wieder Regulierung, Vorgaben, Pflichten. Sie haben vorhin viel von Vertrauen gesprochen, ist das ein Gegensatz?

Rusche: Ich denke, wir alle versuchen zum Beispiel Steuerzahlung zu vermeiden. Aber in einem Ersten Schritt gilt es zu verstehen, dass Steuern der Mechanismus sind, damit der Erfolg des Einzelnen dazu führt, dass das Gemeinwohl genährt wird und wir mit den Steuern Straßen und Bildungsinstitutionen 
finanzieren können. Erstmal dieses Grundverständnis vorauszusetzen, scheint mir wichtig zu sein, bei all den gerade genannten Compliance-Maßnahmen. Denn, dass wir Datenschutz brauchen, dass wir ökologische Schutzmaßnahmen brauchen, dass wir soziale Schutzregelungen brauchen, das müssen wir erstmal voraussetzen - auch als Manager und als Unternehmer - und dann können wir darüber streiten, was für unterschiedliche Unternehmensgrößen angemessen ist. Die Regelungen scheinen mir sehr oft für Großunternehmen gestrickt zu sein und kleine Unternehmen gehen dann in die Knie, weil sie mit diesem Regelungsapparat überfordert sind. Das war ein erster Gedanke.

Ein zweiter Gedanke ist eine alte Unterscheidung, die wir von Kant kennen. Die juristische Regelung Legalität ist zunächst mal etwas ganz Anderes als moralische Legitimität. Während der Zeit des Nationalsozialismus galten ganz viele legale Regelungen, die aber völlig unmoralisch waren und wir müssen aufpassen, denn wir haben uns so sehr daran gewöhnt, dass legale Standards ausreichen. Wir müssen aufpassen, dass wir neben der Verrechtlichung unseres Gesellschafts- und Wirtschaftssystems, die moralische Frage, was denn legitim ist, nicht wegradieren und da setzt CSR ganz wesentlich an. Ich glaube, wir alle müssen aufpassen, dass wir angesichts von Verrechtlichung, angesichts der Kultur der oberflächlichen Meinungsmache, in einer Kultur wo „Social Bots“ Meinungen schaffen und so drehen, dass Wahlen und Volksentscheide wesentlich beeinflusst werden. Wir alle müssen anfangen uns selbst zu fragen, was denn die Grundwerte sind, die uns wichtig sind. Diese Grundlagenreflexion auf das, wofür ich bereit bin einzutreten, mein Fußballverein, mein Glaube, meine Einstellung zu CSR, diese Grundsatzfragen werden in der Gesellschaft, bis auf den Fußball, immer mehr zur Seite gedrängt. Da beginnt für mich CSR-Arbeit in Unternehmen und in den Verbänden. Dass ich erstmal mit den Mitarbeitern gemeinsam dieses Wertefundament erarbeite, um daraus schöpfend, Initiativen zu formulieren.

Domnick: Ja, ich möchte an den ersten Punkt von Herrn Rusche nochmal anknüpfen, das Thema Steuern. Ich glaube, Sie haben das richtig gesagt, wir müssen erstmal alle akzeptieren, dass wir alle Steuern zahlen müssen. Aufgrund der Steuern haben wir in Deutschland ein relativ gutes Sozialsystem. Der ganze Gedanke der CSR kommt ja sehr stark aus dem anglosächsischen Sprachraum, aus Amerika und England, wo eine soziale Infrastruktur in dieser Form gar nicht besteht. Von daher ist es notwendig darauf hinzuwirken, und das ist sicher auch ein kritischer Aspekt mit Blick auf CSR, zu sagen, wir müssen unser Sozialsystem im Grunde über Steuern finanzieren. CSR kann nur ein Add-on sein, zudem was einfach Daseinsfürsorge für die Menschen heißt. Denn, wenn wir umsteuern und sagen CSR ersetzt künftig staatliche Leistungen, dann kommen wir in ganz schwieriges Badwasser. Das sehen wir ja stellenweise bei der Diskussion um die Tafeln, wenn mittlerweile Sozialämter Menschen zu den 
Tafeln schicken. Eigentlich waren die Tafeln dafür gedacht, aus einer Überflussgesellschaft Lebensmittel umzuverteilen, aber nicht als Ersatz für staatliche Leistungen. Da glaube ich, ist es ganz wichtig nochmal darauf zu achten und da nochmal andere Wege zu suchen, was als CSR-Leistungen im Sinne eines Add-ons im sozialen Bereich geleistet werden kann.

Sandfort: Ich würde auch gerne nochmal bei Ihnen (Herr Rusche) anschließen wollen, weil für mich CSR auch ganz maßgeblich eine Wertefrage ist. Wenn wir uns für den Umweltschutz engagieren seitens der Caritas, dann machen wir das, weil wir letzten Endes einen Auftrag dafür haben unsere Schöpfung zu bewahren. So könnte man das theologisch ausdrücken. Wir setzen uns für Mitarbeiterinnen und Mitarbeiter deswegen ein, weil wir glauben, dass die Menschen, die bei uns beschäftigt sind, unter guten Arbeitsbedingungen tätig sein sollen. Wenngleich, das ist ja auch schon angesprochen worden, wir in großen Teilen unter schwierigen Rahmenbedingungen leiden, was aber nicht damit zusammenhängt, dass wir nicht als Dienstgeber für gute Rahmenbedingungen zu sorgen versuchen, sondern weil sie uns in Teilen von außen aufgetragen und aufgesetzt werden. Ein ganz zentraler Aspekt ist die Frage, inwieweit wir CSR herunter brechen in die Mitarbeiterschaft hinein. Es muss aus dem Unternehmen heraus leben. Es macht wenig Sinn, wenn wir CSR als verbandliche Strategie implementieren, ohne die Mitarbeitenden mit auf den Weg zu nehmen. Das ist ein ganz entscheidender Punkt und letzten Endes sind auch alle Mitarbeiterinnen und Mitarbeiter sehr dafür sensibilisiert, was der Verband an CSRMaßnahmen initiiert und ob er diese auch glaubwürdig umsetzt. Ich denke, dass das für uns als Unternehmen sehr wichtig ist, als glaubwürdiger Arbeitgeber wahrgenommen zu werden, der ernst nimmt, was er sich auf die Fahne schreibt und dies dann in der Praxis auch lebt.

Wippermann: Ich wollte nochmal eine kurze Ergänzung machen zu dem Thema Steuern zahlen oder CSR oder Widersprüche, die es da vielleicht gibt. Das mache ich an dem Beispiel Stiftungen. Wir sehen die Stiftungen, die ja ein Modell in Deutschland sind, um auch Unternehmensgewinne sinnvoll, sozial, ökologisch, wie auch gedacht, anzulegen, auch kritisch. Wenn man sich allein die Bill-Gates-Stiftung anschaut. Diese hat mehr Budget als sämtliche Staaten der Subsahara in Afrika in ihren Gesundheitsfonds, aber sie ist sozusagen undemokratisch. Niemand kann da reinregieren. Es ist Bill Gates, der selbst mit seiner Frau bestimmt, wofür das Geld ausgegeben wird. Das kann man jetzt zwar als eine sozial wohltätige Leistung sehen, auf der anderen Seite hat Gates über das Stiftungskapital Gelder entzogen, die sonst in Steuergelder gegangen wären und damit einer demokratischen Kontrolle unterlegen hätten und man hätte diese Gelder dann demokratisch ausgegeben. Auch das finde ich einen 
wichtigen Punkt zu sehen, wo CSR über Stiftungen ein Moment ist, wo man Geld den Steuergeldern entzieht.

Diel: Herr Scholz teilt die Einschätzung vollumfänglich oder nur in Teilen?

Scholz: Immer nur in Teilen natürlich.

Diel: Dann hätte ich ihren Gesichtsausdruck richtig gelesen.

Scholz: Ich finde, wir haben einen wichtigen Punkt angesprochen: CSR als Substitution zum klassischen staatlichen Handeln, dass also Unternehmen oder andere Organisationen Staatsaufgaben übernehmen. Sie, Herr Domnick, hatten angesprochen, dass die Tafeln zu dem Zweck gegründet wurden, der Überflussgesellschaft und der Lebensmittelverschwendung entgegenzuwirken. Jetzt werden diese Einrichtungen zum Teil missbraucht, weil wir ein Staatsversagen haben und die vielen hungrigen Menschen dort versorgt werden sollen. Das ist nicht die Idee von CSR oder die Idee von solchen Organisationen. Das ist doch erstmal Staatsaufgabe. Trotzdem haben wir im Moment scheinbar ein Thema, dass der Staat in vielen Bereichen versagt. Also nicht nur bei den neuen Technologien oder bei den neuen Geschäftsmodellen, sondern auch bei ganz klassischen Punkten, zum Beispiel die notwendige Anzahl, Quantität und Qualität an Pflegerinnen und Pflegern zur Verfügung zu stellen. Dann soll es natürlich nicht die Verantwortung der Unternehmen sein, diese Aufgaben zu übernehmen. Und Sie, Frau Wippermann, hatten das weitergeführt und haben gesagt, außerdem sind die Unternehmen und die Stiftungen überhaupt nicht legitimiert zu entscheiden, was sie da tun sollen. Also warum soll es eine „Bill und Melinda Gates Foundation“" sein, die sagt: „Wir kümmern uns jetzt beispielsweise um Krebs, aber nicht um HIV. Wir kümmern uns um die Länder des globalen Südens, aber nicht um die Länder in Europa.“ Wir müssen über die Legitimierung von Unternehmen, die dann quasi politisch handeln, nachdenken. Und Herr Rusche hatte in diesem Kontext auch schon angesprochen, dass wir „das Richtige“ tun müssen, wir müssen legitim handeln, moralisch legitim handeln und das ist ein Unterschied zur Legalität. Die Frage ist dann: Was ist moralisch legitimes Handeln? Also hier, in diesem Raum, scheint es relativ einfach zu sein: Da hängt ein Kreuz und wir haben mehrere Kirchenvertreter hier - wir beziehen uns auf den christlich-jüdischen Wertekanon. In einer pluralistischen Gesellschaft scheint mir das allerdings komplexer zu sein. Was machen wir denn, wenn wir viele Muslime bei uns haben oder Menschen ohne Glaubensbekenntnis? Was ist denn dann „das Richtige“? Ich glaube, was moralisch legitim ist, müssen wir im Kontext von CSR ausverhandeln. Da gibt es ja Vorschläge, beispielsweise Multi-Stakeholder-Debatten. Die müssen aber auch in einer besonderen Art und Weise geführt werden: Sie müssen inklusiv 
sein und es muss Fairness miteinander geben und dann können wir uns vielleicht auf etwas einigen. Aber, und Herr Rusche hatte das auch schon angeführt, dass wir mit unserem eurozentrischen und christlich geprägten Weltbild sagen: „Das und das ist ,das Richtige“, das und das ist legitim.“ Das scheint mir der falsche Zugang zu sein.

Sandfort: Ich würde gerne noch etwas dazu sagen. Stichwort Staatsversagen. Ich bin in Teilen ganz bei Ihnen. Vielleicht ist Ihnen bekannt, wie viele Regelungen wir in der Altenhilfe haben. Wir haben etwa 38 oder 40 verschiedene Normen und Gesetze, die wir einhalten und umsetzen müssen. Das zieht so viel Dokumentationsaufwand nach sich, dass wesentliche Zeit für die Menschen, die wir eigentlich pflegen sollen, nicht zur Verfügung steht. Ich würde aber noch ein zweites Thema an dieser Stelle nennen. Ein ganz anderer Bereich, der uns aber auch sehr berührt im Moment, auch als Wohlfahrtsverbände, ist die Wohnungsnot in Deutschland. Das Thema kennen Sie alle, gerade in den städtischen Ballungszentren ist die Wohnungsnot riesengroß. Und ein Grund dafür hängt damit zusammen, dass wir im Jahr 1990 noch ungefähr 5.000 Baunormen hatten. Heute haben wir 20.000 Baunormen. Wir regulieren uns über, wir schaffen immer mehr Normen, um damit eine vermeintliche Sicherheit zu schaffen, die aber in Wirklichkeit nicht existiert. Das Problem ist, dass diejenigen, die diese Regelungen anwenden sollen, in der Kommunalverwaltung, im Bauamt, völlig überfordert sind mit dieser Situation und deswegen keine Entscheidung treffen, weil sie jede Norm berücksichtigen und beachten müssen. In Folge dessen dauern die Entscheidungsprozesse länger und eine weitere Folge ist, dass Entscheidungen nicht getroffen werden, weil wir momentan sehr häufig erleben, dass Menschen mit einer Entscheidung nicht einverstanden sind und den Klageweg beschreiten. Das sind große Probleme, die damit zusammenhängen, dass wir uns auf der einen Seite völlig über regulieren und die Frage nach ethisch begründbaren Entscheidungen auf der anderen Seite immer mehr in den Hintergrund gerät.

Diel: Okay, ich versuche mal eine Art Zusammenfassung: Wir hatten zwei große Fragen, die miteinander zusammenhängen, zu klären. Sie haben gefragt, was die Legitimation von CSR ist und warum ein Unternehmen sich im Bereich CSR engagieren und warum es im Sinne von CSR handeln sollte. Sie, Herr Scholz, haben in Ihrem Vortrag von deontologischen Begründungen gesprochen, und Sie, Herr Sandfort, haben von den Werten gesprochen. Ich würde sagen, wir lassen das jetzt an dieser Stelle für die Sozialverbände mal so stehen. Denn das ergibt sich ja im Prinzip auch aus den Gedanken und Werten, die hinter dem sozialen Verband stehen. Es braucht nicht anders begründet zu werden. Für die freie Wirtschaft oder für die Profitunternehmen muss ich 
allerdings eine andere Begründung haben. Da komme ich mit einer deontologischen Argumentation nicht weiter. Der typische Unternehmer wird fragen, was habe ich davon, was ist die Wirkung. Ich glaube, dass wir diese Fragen in der letzten viertel Stunde eigentlich schon ganz gut beantwortet haben, weil wir viele Argumente geliefert haben, was ein Unternehmen davon hat, wenn es so handelt. Und zwar geht es über das was sie „Green washing“ genannt haben hinaus. Stattdessen tue ich meinem Unternehmen, meiner Struktur tatsächlich etwas Gutes, wenn ich die Mitarbeiter auch in diesem Sinne mitnehme. Wenn ich etwas bieten kann, was über Geld hinausgeht, nämlich ein gutes Arbeitsumfeld und den Mitarbeitern ein gutes Gefühl, das sie in meinem Unternehmen arbeiten, dass sie sich gemeinsam einsetzen. Das würde ich jetzt mal als Begründung stehen lassen und sagen: jawohl jetzt haben wir die Leute überzeugt, CSR ist eine tolle Sache, da machen wir mit, das nutzt uns selbst, das nutzt der Gesellschaft und dann stellt sich natürlich die nächste große Frage: Wie kann man das denn machen? Sie haben ja gesagt, das klassische Beispiel ist, wir spenden für ein Projekt - das ist ja auch ein bisschen CSR. Aber wie kann es denn darüber hinausgehen? Wie kann das aussehen? Was wären so praktische Ideen CSR umzusetzen?

Domnick: Ein ganz praktisches Projekt, was wir jetzt im Februar gestartet haben, nennt sich „Öko-Profit“. Das heißt wir haben Arbeitsgemeinschaften und Workshops in der Stadt Mainz mit verschiedenen Unternehmen, wo wir (die Idee kommt aus Österreich) als Unternehmen uns zusammensetzen und schauen, wie können wir nachhaltiger wirtschaften und gleichzeitig einen betriebswirtschaftlichen Nutzen dadurch haben. Energiemanagement, Abfalltrennung, Abfallreduzierung, im Einkauf nochmal Verbünde schaffen - da passieren interessante Entwicklungen. In einem dieser Workshops haben wir ein großes Mainzer Unternehmen, Werner und Mertz das die Frosch-Reinigungsmittel herstellt und ein großes Reinigungsunternehmen mit dabei. Es gab dann eine Vereinbarung, dass die Teilnehmenden an diesem Workshop künftig mit den Frosch-Produkten, die umweltfreundlich sind, die nachhaltig produziert werden, reinigen. Auch das große Reinigungsunternehmen, das auch in mehreren kirchlichen Einrichtungen tätig ist und darüber auch nochmal einen Nachhaltigkeitseffekt entstehen lässt. Ich glaube, das sind Punkte, wo es darum geht, wie Unternehmen an dieser Stelle enger zusammenarbeiten können, um nachhaltiger zu sein.

Zum zweiten ist der Blick auf die Mitarbeiterinnen und Mitarbeiter zentral. Ich glaube, dass wir auf einen Fachkräftemangel in allen Bereichen zusteuern und von daher ist es notwendig perspektivisch auch zu schauen, was wir unseren Mitarbeitern bieten können. Ich glaube, da haben wir als soziale Organisation eine ganz gute Ausgangssituation, weil viele unserer Mitarbeiter wissen, 
dass sie im sozialen Bereich weniger verdienen, aber dafür eine sinnvolle Tätigkeit machen, die wertorientiert ist und wo man hilft. Vor längerer Zeit war ich bei einem Vortrag von Wendelin Wiedeking, dem früheren Vorstandschef von Porsche. Bei mir saß der Leiter eines Altenheims, der fragte: „Herr Domnick wollten Sie in einem Unternehmen arbeiten, wo man eigentlich nutzlos ist?" Das fand ich eine super Aussage, wo ich dachte, dieser Mann da vorne verdient das zwanzigfache von dem, was wir verdienen oder noch mehr und der Altenheimleiter sagt: „Dass was ich tue ist werthaltig. Wollten Sie für Ihn arbeiten, für Porsche? Also wer braucht einen Porsche?“

Diel: Erstaunlich viele Menschen beantworten diese Frage ja mit: „Ich“.

Domnick: Ich sagte, wer braucht ihn. Wer mag ihn, ist was anderes.

Rusche: Die Sinnstiftung, tue ich etwas Sinnvolles, scheint mir die höchste Übung in der Unternehmenskultur zu sein. Für sich als Unternehmer, als Manager oder als Abteilungsleiter klar zu machen, was tue ich hier in sinnstiftender Art und Weise und das so zu formulieren, dass die Mitarbeiter diese Sinnstiftung als eigene Aufgabe begreifen ist eine große Herausforderung. Allerdings, wir hatten gerade eben in der Kaffeepause kurz dieses Thema, ist Sinnstiftung alleine noch kein ethisches Kriterium. In einer mafiösen Unternehmung sind auch alle der Überzeugung, dass sie Sinn stiften. Da gibt es auch sehr klare moralische Kommunikationsregeln und Sie glauben gar nicht wie sinnstiftend Nationalsozialisten ihr Tun empfunden haben. Das heißt, wir bedürfen einer ethischen Grundlagenreflexion.

Sandfort: So ist es. Und mit Blick auf die Vergütung vielleicht noch eine Randbemerkung, denn wir haben natürlich auch in der Pflege ein Imageproblem, das ist ja sehr wohl bekannt. Aber es ist, finde ich, sehr wichtig nochmal deutlich zu machen, dass Caritas und Diakonie und auch die anderen Wohlfahrtsverbände tariflich bezahlen und sich damit deutlich von manch anderen in der Branche abheben.

Aber ich wollte etwas Anderes sagen, Sie Herr Diel, fragten, wo es Anknüpfungspunkte zum Thema CSR in unserem Unternehmen gibt. Ich hatte eingangs darüber berichtet, dass wir ein von der Deutschen Bundesstiftung Umwelt gefördertes Projekt umgesetzt haben mit dem Titel „Zukunft einkaufen", indem es um nachhaltige Beschaffung geht. Wir haben also verschiedene Modelleinrichtungen besucht und geschaut, wie wird da eigentlich die Beschaffung organisiert. Unter welchen Kriterien werden Produkte eingekauft? Werden die saisonal eingekauft? Werden sie vor Ort eingekauft oder werden sie importiert? Welche Gütesiegel finden Anwendung? Diese ganzen Überlegungen haben wir dann zusammengetragen und zusammengefasst in Beschaffungsleitlinien. Das heißt, wir haben unseren Einrichtungen und Diensten Leitlinien an die Hand gegeben, auf welche Punkte bei der Beschaffung zu achten ist und das dann weitestgehend in Form einer Beschaffungsordnung umgesetzt. 
Hier haben wir dann in Teilen auch Vorgaben gemacht: zum Beispiel dahingehend, dass unsere Mitarbeiter grundsätzlich bei Dienstfahrten prüfen müssen, ob sie mit dem öffentlichen Personennahverkehr ihr Ziel erreichen oder das Auto nehmen müssen. Oder indem wir beispielsweise unsere Einrichtungen verpflichtet haben, die sogenannte Caritasbox aufzustellen. Das ist eine Box, in der entsorgt man alte Druckerkartuschen, die dann anschließend recycelt werden. Oder wir haben unseren Einrichtungen und Diensten drei verschiedene Alternativen an Stromanbietern zur Verfügung gestellt, die aber nachweislich Strom so produzieren, dass es umweltgerecht und nachhaltig ist. Und wir sorgen natürlich auch dafür, dass wir vor Ort Fairtrade-Produkte zum Einsatz bringen und schauen, wo wir den Kaffee einkaufen oder das wir darüber nachdenken, wie bestimmte Dinge produziert wurden, soweit wir das denn nachvollziehen können.

Diel: Frau Wippermann.

Wippermann: Ja, ich wollte noch zwei kleine Beispiele zu Ihrer Frage nennen. Im Vorfeld der heutigen Tagung habe ich mich mit einer Kollegin mit Behinderung unterhalten und sie nannte mir dann ein Beispiel. Sie war mal in einem Wohnheim und dann kamen Mitarbeiter der Deutschen Bank zum Freiwilligen Tag und haben dann alle mit dem Rollstuhl zu einem Ausflug mitgenommen. Nachher wurden sie für ein Foto für die Mitarbeiterzeitung der Deutschen Bank abgelichtet. Sie sagte, sie saß da wie ein begossener Pudel. „Zu was war ich da plötzlich da? Ich war das Objekt einer sozialen Aktion des Deutsche Bank-Mitarbeiters oder zu was war ich hier eigentlich." Sie hat gesagt, ab diesem Moment hat sie nie wieder an solchen Freiwilligen-tagen der Deutschen Bank teilgenommen, also sich geweigert, weil sie merkte, sie wird da instrumentalisiert. Das war ein Beispiel. Zweites Beispiel: Es gibt ein Projekt „Patenschaftsmodell Offenbach“. In dem Projekt stellen Unternehmen (u.a. auch die Deutsche Bank) Mitarbeiter zeitlich frei um Jugendliche ab der 8. Klasse mit Haupt- bzw. Realschulabschluss zu begleiten. Da wird Geld gegeben, da wird den Mitarbeitern Zeit gegeben und es werden Räumlichkeiten zur Verfügung gestellt. Die machen echt gute Arbeit muss ich sagen. Die unterstützen Jugendliche aus Offenbacher Hauptschulen, die sich melden und unterstützen diese mit professionellen Kräften ehrenamtlich. Ich wollte zwei kleine Geschichten benennen: beide von einer großen Bank (mit)finanziert, aber mit sehr unterschiedlicher Wirkung.

Diel: Herr Scholz, ihr Abschlussplädoyer.

Scholz: Mein Abschlussplädoyer. Zunächst gehe ich kurz darauf ein, was Frau Wippermann gesagt hat. Was die Deutsche Bank dort macht, nämlich Mitarbeiterinnen und Mitarbeiter, verzeihen Sie, wenn ich es so ausdrücke, dort 
„hinzukarren“ um dann Pflegebedürftigen zu helfen, hat ja vielleicht zwei Motive: Zum einen hilft das den Deutsche Bank-Angestellten, sich wertvoll zu fühlen: Sie haben jetzt tatsächlich einen Nutzen generiert, auch innerhalb ihres Jobs können sie sich irgendwo einsetzen. Und zum anderen unterstützt es natürlich das Reputationsmanagement der Deutschen Bank und gleichzeitig hilft es ja vielleicht doch Menschen. Also dort wird der Fachkräftemangel sozusagen subsituiert. Die Frage ist, ob es hier einen Trade-off gibt? Man könnte wohlwollend sagen: „Da passiert etwas“. Trotzdem habe ich ja vorhin eine Unterscheidung zwischen CSR und Philanthropie gemacht und ich glaube, dabei würde ich auch bleiben. Als Philanthropie würde ich verstehen: „Business as usual" und am Ende des Geschäftsjahres gibt man dann irgendetwas ab. Egal, ob das mit dem Kerngeschäft zu tun hat oder nicht. Es kann sein, dass sich eine Unternehmerin, ein Unternehmer dazu bemüßigt fühlt, weil das ihrem Glauben entspricht. Das hat aber noch nichts mit CSR zu tun, weil es nämlich im Unternehmen an sich nichts verändert. Stattdessen wird weiter „Business as usual" gemacht und hinterher werden, man könnte fast sagen, ein paar Almosen gegeben oder Ablass eingefordert. Trotzdem gibt es auch interessante Ansätze, die ähnlich wie bei der Deutschen Bank, strategisch wertvoll sein können. Ich nenne Ihnen auch ein Beispiel: Was Coca-Cola gerade macht ist ein ganz tolles Beispiel. Ob es ausreichend ist oder nicht, müssen Sie entscheiden. Coca-Cola hat ein Projekt das heißt ,,5by2020“. Da fördert Coca-Cola bis 2020 fünf Millionen Frauen in sogenannten Entwicklungsländern. Sie geben den Frauen Mikrokredite, ganz kleine Beträge, und sie trainieren ihnen einige BWL-Fähigkeiten an. Warum macht Coca-Cola das? Nicht, weil Coca-Cola jetzt eine soziale Ader für sich entdeckt hat, sondern weil sie hoffen, dass sich diese Frauen dann selbstständig machen. Das machen die meisten auch (es ist ein gut untersuchtes Programm) und gründen dann kleine „Points of sales“. Das ist super für die Region. Da gibt es dann nämlich diese kleinen Kioske und Coca-Cola hat idealerweise ein neues Sales-Netzwerk. Fünf Millionen „Points of sale". Problematisch dabei finde ich es, dass Coca-Cola auch hier überhaupt nichts am Geschäftsmodell ändert. Das Geschäftsmodell von Coca-Cola bleibt nach wie vor Zuckerwasser zu verkaufen. Dieses Zuckerwasser ist nachgewiesener Weise sehr, sehr schädlich und nichts wird am eigenen Geschäftsmodell geändert. Das sind diese „Win-Win“ und „Win-Lose Cases“ von denen ich vorhin gesprochen habe und ich glaube, da müssen zumindest wirtschaftliche Unternehmen sich überlegen, was sie da eigentlich tun. Was ist denn eigentlich der Sinn des Geschäftes? Ist Zuckerwasser zu verkaufen wirklich die Art und Weise oder müssen wir darüber nachdenken, was wir sonst noch tun können? Das ist einfacher gesagt, als getan. Diese strategischen Ansätze haben also Grenzen und ich glaube, Collective Action könnte diesen Trade-off von ,ich will das Richtige tun, aber ich kann es nicht alleine" zumindest minimieren. 
Das wäre eine Idee dazu. Ich möchte abschließend noch sagen, weil Herr Sandfort das vorhin angesprochen hat: Herr Sandfort, Sie haben gesagt, wir haben $\mathrm{zu}$ viel Regulierung. Ich glaube auch, dass wir in vielen Bereichen zu viel Regulierung haben. Aber gleichzeitig haben Sie bei Ihrem Anschlussstatement gesagt: „In unserem Unternehmen haben wir Bestellrichtlinien erlassen, wir haben X und Y getan“. Das heißt, Sie haben auch formalisiert. Ich glaube, umso komplexer Unternehmen werden, umso mehr wird auch formalisiert. Nun gibt es gleichzeitig einen Unterschied, Herr Rusche hat das sehr schön gesagt, zwischen Compliance, also Legalität, „das Richtige“ machen, sei es nach außen, gegenüber der Gesellschaft alle Gesetze einzuhalten oder innerhalb eines Unternehmens alles richtig zu machen und „das Richtige“ tun, also Integrität. Da gibt es einen Unterschied, aber trotzdem ist Formalisierung nicht immer schlecht.

Domnick: Ein Satz zum Thema Collective Action. Es ist wichtig, dass Organisationen sich zusammenschließen, aber auch Betroffene beteiligen.

Scholz: Ja, unbedingt.

Domnick: In dem Moment passieren nämlich genau solche Geschichten nicht, von denen Frau Wippermann berichtet hat.

Sandfort: Herr Professor Scholz Sie haben natürlich Recht, wir haben Beschaffungsleitlinien, aber hier gilt es wie in der Pädagogik. Wenige Regeln, aber die bitte anwenden.

Scholz: Vollkommen richtig.

Diel: Meine Damen und Herren vielen herzlichen Dank. 


\section{Angaben zu den Autorinnen und Autoren}

Heike Herrmann, Prof. Dr. Phil., ist Dipl. Soz., promovierte in Soziologie. Seit 2006 ist sie Professorin für Soziale Arbeit im Sozialraum und Sozialmanagement an der HS Fulda. Seit 2011 Sprecherin der Sektion Stadt- und Regionalsoziologie der Deutschen Gesellschaft für Soziologie (DGS). An den Schnittstellen zwischen Wissenschaft und Forschung, zivil-gesellschaftlichen, (privat-)wirtschaftlichen Akteuren und politisch-administrativem System setzt sie sich für eine Integrierende Stadtentwicklung (ISE) ein und gründete/leitete den gleichnamigen Themenschwerpunkt am Forschungsinstitut für gesellschaftliche Weiterentwicklung (FGW) in Düsseldorf. Sie ist zudem (Mit-) Gründerin des fakultätsübergreifenden wissenschaftlichen Zentrums für Gesellschaft und Nachhaltigkeit (CeSSt) der Hochschule Fulda. Über 10 Jahre war sie zudem Vorsitzende eines freien Trägers der Kinder- und Jugendhilfe. Schwerpunkte in Lehre und Forschung sind u.a. Themen der Sozialen Ungleichheit, der Stadt- und Raumforschung, des Sozialmanagements und CSR/Corporate Citizenship.

Kontakt: heike.herrmann@sw.hs-fulda.de

Karl-Hans Kern, Dipl.-Sozialpäd. (FH), arbeitete vier Jahre für Caritas International in der Entwicklungszusammenarbeit in einem Flüchtlingslager in Honduras. Zurück in Deutschland, verantwortete er zu Beginn der 90er Jahre als Referent das Themenfeld Migration im Caritasverband der Diözese Rottenburg-Stuttgart e.V. Im Jahr 2000 wechselte er in das Aufgabenfeld „Unternehmenskooperationen", absolvierte an der Evangelischen Fachhochschule Darmstadt eine Weiterbildung ,Soziales Marketing“ und war bei der Caritas u.a. für den Aufbau und die Durchführung der bundesweiten Unternehmenskooperation der Caritas mit dem Fahrzeugimporteur Hyundai Motor Deutschland $\mathrm{GmbH}$, Offenbach verantwortlich. 2011 übernahm er die Leitung des CSRKompetenzzentrums im Deutschen Caritasverband war bis November 2018 bundesweit Ansprechpartner für Unternehmen und die Caritas zu CSR-Themen. Seit Dezember 2018 ist er im Ruhestand und begleitet weiterhin Caritasverbände und die Malteser Werke gGmbH in Köln bei dem Aufbau und der Entwicklung von CSR-Aktivitäten. 
Maria Riegler, MSc, ist wissenschaftliche Mitarbeiterin am Center for Corporate Governance \& Business Ethics an der FHWien der WKW und PhD-Studentin an der MODUL University. Neben beruflichen Tätigkeiten für Forschungsinstitute, Unternehmen und NGOs absolvierte sie ein Masterstudium der Sozioökonomie an der Wirtschaftsuniversität Wien und ein Bachelorstudium an der WU Wien und der Universität Lund in Schweden. In ihrer Dissertation beforscht sie die Entstehung und Entwicklung von Collective Action und Private-Governance-Initiativen.

Kontakt: maria.riegler@fh-wien.ac.at

Thomas Rusche, PD Dr. rer. pol. und Dr. phil., ist geschäftsführender Gesellschafter der SØR Rusche GmbH mit über 50 Niederlassungen von Sylt bis Garmisch, Marktführer im Premiumsegment der deutschen Kleidungskultur. Er führt das Familienunternehmen in vierter Generation. Zudem ist er 1. Vorsitzender des Hilfswerks Schwester Petra, Vorstandsmitglied und deutscher Koordinator der päpstlichen Stiftung 'Centesimus Annus - Pro Pontifice', Vatikan; Mitglied im Kuratorium des Westfälischen Friedenspreises, Münster sowie Ritter vom Heiligen Grab zu Jerusalem. Thomas Rusche ist Kurator des Hans-Jonas-Zentrums der Universität Siegen sowie Gründungsmitglied und Leiter der angeschlossenen Forschungsgruppe EWD (Ethik und Wirtschaft im Dialog). An der WHU - Otto Beisheim School of Management Vallendar unterrichtet er Unternehmensethik. 2018 hat er mit einer unternehmensethischen Schrift an der Universität Siegen habilitiert und seit 2019 dort am philosophischen Institut seine Tätigkeit als Privatdozent aufgenommen.

Christoph Schleer, Dr. rer. pol., studierte Wirtschaftswissenschaften an der Otto-von-Guericke-Universität Magdeburg und der Albert-Ludwigs-Universität Freiburg. 2014 promovierte er an der Gottfried Wilhelm Leibniz Universität Hannover mit einem Stipendium des imug-Instituts für Markt-Umwelt-Gesellschaft. Für seine wissenschaftlichen Leistungen wurde er 2009 mit dem RalfBodo-Schmidt-Preis der Universität Freiburg ausgezeichnet. Seit 2014 ist Dr. Schleer bei SINUS als Studienleiter und seit 2017 als Senior Research \& Consulting tätig. Für die SINUS-Akademie arbeitet er als gefragter Referent. Seine Forschungs- und Arbeitsschwerpunkte sind: Umwelt-, Klima- und Naturbewusstsein, Corporate Social Responsibility (CSR), Nachhaltiger Konsum, Nachwuchsmarketing und Migrationsforschung.

Kontakt: christoph.schleer@sinus-institut.de 
Markus Scholz, FH-Prof. Dr., ist Inhaber des Stiftungslehrstuhls für Corporate Governance \& Business Ethics und leitet das Center for Corporate Governance \& Business Ethics sowie das Center for Strategy \& Competitiveness an der FHWien der WKW. Zudem forscht und lehrt er am INSEAD Social Innovation Centre sowie an der London School of Economics. Markus Scholz ist Botschafter der Giving Voice To Values-Initiative und berät eine Reihe von internationalen öffentlichen und privaten Organisationen mit einem Fokus auf Environmental, Social and Governance (ESG) Themen.

Kontakt: Markus.Scholz@fh-wien.ac.at

Bettina Stoll, Prof. Dr. phil., ist Dipl.-Päd. (Univ.), Dipl.-Sozialpäd. (FH), promoviert in Soziologie. Seit 2013 Professorin für Sozialmanagement/Hochschule Fulda. In ihrer langjährigen beruflichen Praxis (z.B. in international agierendem Großunternehmen; als Geschäftsführerin einer Bildungsinstitution) sind (internationale) Projekte, Kooperationen und Maßnahmen im Kontext von „Corporate Social Responsibility“ von großer Relevanz. Schwerpunkte in Lehre und Forschung sind u.a. Sozialmanagement, „Soziales und Wirtschaft", CSR/Corporate Citizenship, Soziale Unternehmen/Social Entrepreneurship. Sie ist u.a. Leitungsmitglied im fakultätsübergreifenden wissenschaftlichen Zentrum für Gesellschaft und Nachhaltigkeit (CeSSt) der Hochschule Fulda; Mitglied des erweiterten Vorstandes der Bundesarbeitsgemeinschaft für Sozialmanagement/ Sozialwirtschaft; ehrenamtl. wissenschaftliche Beirätin für EMASplus.

Kontakt: bettina.stoll@sw.hs-fulda.de 


\section{Beiträge zur Sozialraumforschung}

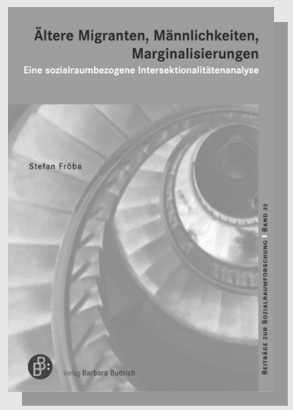

Band 22

2019 211 Seiten • Kart. • $29,90 €(D) \cdot 30,80 €(A)$

ISBN 978-3-8474-2337-9

eISBN 978-3-8474-1508-4

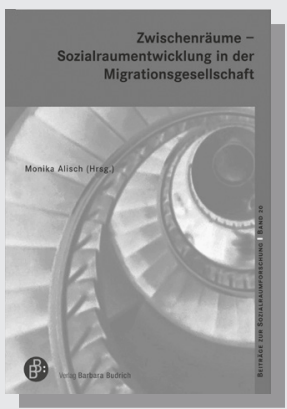

\section{Band 20}

2019 204 Seiten • Kart. • $28,00 €(D) \cdot 28,80 €(A)$ ISBN 978-3-8474-2276-1 eISBN 978-3-8474-1308-0

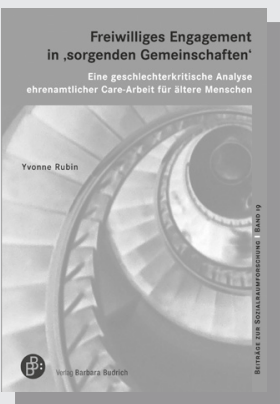

Band 19

2018 - 260 Seiten • Kart. • $34,00 €(D) \cdot 35,00 €(A)$ ISBN 978-3-8474-2242-6 elSBN 978-3-8474-1307-3

In der Schriftenreihe „Beiträge zur Sozialraumforschung“ werden Sammelbände und Monografien veröffentlicht, die Prozesse der Sozialraumentwicklung und -organisation in Beziehung setzen zu aktuellen, gesellschaftlich hoch relevanten Themen wie Inklusion, Segregation, Migration, Gender und Fragestellungen aus den verschiedenen Arbeitsfeldern Sozialer Arbeit.

Die Reihe dokumentiert die lebendige und vielfältige Sozialraumforschungslandschaft.

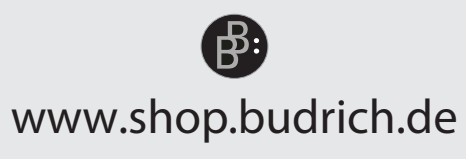


Unternehmen in Profit- und Sozialwirtschaft tragen ökonomische, ökologische und soziale Verantwortung, auch als Corporate Social Responsibility bezeichnet. Um dieser - ggf. auch durch Kooperationen - entsprechen zu können, bedarf es Aushandlungsprozesse, kluger Wirtschafts- und Sozialkonzepte, Unternehmenskonzepte und Wege zur Verankerung des nachhaltigen Leitbilds in den Institutionen. Der Band setzt dazu Impulse für die Profit- und Sozialwirtschaft.

Die Herausgeberinnen:

Prof. Dr. Bettina Stoll, Fachbereich Sozialwesen, Hochschule Fulda

Prof. Dr. Heike Herrmann, Fachbereich Sozialwesen, Hochschule Fulda

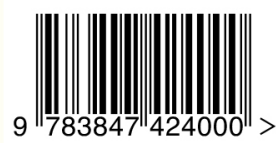

www.budrich.de 